\title{
Exploring, modelling, and simulating \\ The Feeling of Comfort \\ in residential settings
}

By

\section{GERMÁN MOLINA}

\begin{abstract}
A thesis submitted to
VICTORIA UNIVERSITY OF WELLINGTON IN FULFILMENT OF THE REQUIREMENTS FOR THE DEGREE OF DOCTOR OF PHILOSOPHY
\end{abstract}

Victoria University of Wellington 
Ph.D. candidate

Supervisors (alphabetic)
Germán Molina

Dr Michael Donn

Dr Micael-Lee Johnstone

Dr Casimir MacGregor 


\section{ACKNOWLEDGEMENTS}

I have heard that a PhD journey is one that we take alone. I guess you can choose to do that, but it is certainly not what I did. My PhD was only possible thanks to many people to whom I owe this degree.

First and foremost, my wife. As far as I am concerned, this was our journey. Together we decided whether to search and apply for scholarships, we discussed where we would be willing to move to, and chose to accept the position I was offered. Her support allowed me to get to the end of this. The same applies to the rest of my family, who supported me from Chile; my friends, those living in Chile and New Zealand; and my children, although these are too young to know how valuable their contribution is.

Other people participated more directly in the writing and research tasks of this journey. They had to go through the pain of reading and discussing my writings. I refer, of course, to my supervisors. They not only helped me answer academic and non-academic questions but also personally guided me through this, at times, overwhelming process. It is hard to express how much I learned from them.

I am also deeply thankful to Victoria University of Wellington, who funded my studies; and to all the 42 participants-from Chile and New Zealand-who dedicated some of their time to talking about weird things with me. Without their help, I would have had nothing to write about. 


\begin{abstract}
The fact that comfort is a subjective state of the mind is widely accepted by engineers, architects and building scientists. Despite this, capturing all the complexity, subjectivity and richness of this construct in models that are useful in building science contexts is far from straightforward. By prioritizing usability, building science has produced models of comfort (e.g., acoustic, visual and thermal) that overly simplify this concept to something nearly objective that can be directly associated with people's physiology and measurable and quantifiable environmental factors. This is a contradiction because, even if comfort is supposed to be subjective, most of the complexity of "the subject" is avoided by focusing on physiology; and, even if comfort is supposed to reside in the mind, the cognitive processes that characterize the mind are disregarded. This research partially mitigates this contradiction by exploring people's non-physical personal factors and cognition within the context of their comfort and by proposing a way in which they can be incorporated into building science research and practice. This research refers to these elements together-i.e., people's nonphysical personal factors and cognition—as "the mind".

This research proposes a new qualitative model of the Feeling of Comfort that embraces "the mind". This model was developed from the results of a first study in which 18 peoplefrom Chile and New Zealand-were asked to describe "a home with good daylight" and "a warm home" in their own words. These results were then replicated in a second study in which another group of 24 people — also from Chile and New Zealand_-described "a home with good acoustic performance", "a home with good air quality" and "a pleasantly cool home". The Feeling of Comfort model not only was capable of making sense of the new data (gathered in this second study) but also proved to be simple enough to be useful in the context of comfort research and practice. For instance, it guided the development of a quantitative Feeling of Comfort model and also of a prototype building simulation tool that embraces "the mind" and thus can potentially estimate people's Feeling of Comfort.

This research concludes that embracing "the mind" is not only possible but necessary. The reason for this is that "the mind" plays a significant role in the development of people's comfort. Thus, theories and models of comfort that ignore it fail to represent properly the concept of comfort held by the people for whom buildings are designed. However, incorporating "the mind" into building science's research and practice implies embracing tools, research methods and conceptual frameworks that have historically not been used by such a discipline. Specifically, it concludes that building science should normalize a more holistic view of comfort and perform more exploratory and qualitative research.
\end{abstract}




\section{PREFACE}

"This is how they survive. You must know this. You are too smart not to know this. They paint the world full of shadows, and then tell their children to stay close to the light. Their light, their reasons, their judgments. Because in the darkness, there be dragons. But it isn't true. We can prove that it isn't true. In the dark there is discovery, there is possibility, there is freedomin the dark-once someone has illuminated it. And who has been so close to doing it as we are right now?"

CAPTAIN FLINT, BLACK SAILS

Kuhn (2012, p. 5) describes normal science as "a strenuous and devoted attempt to force nature into the conceptual boxes supplied by professional education". He argues that the world view of researchers-inherited from educators and previous scientists - shapes not only the way they gather and interpret data but also the very questions they ask. Nevertheless, periods of normal science often produce "anomalies" and "curiosities" that do not easily fit into scientists' conceptual boxes. After some time, when the pile of "anomalies" gets excessively big, science goes through a debate that leads to the replacement of these boxes. This dissertation argues that for building science's comfort research-such a debate is worth having now. Furthermore, it proposes an alternative "box" for researchers to use... although, I am not arrogant enough to say that this box is the future.

Proposing a new conceptual box implies that, instead of critiquing the accuracy of current comfort indices by using traditional techniques, this dissertation questions the very definitions of comfort used in research and practice. In doing so, it draws knowledge from other disciplines and utilizes methods and concepts that are not necessarily familiar to building scientists. I am afraid there was no other way of doing this, as getting out of the box requires leaving some of your tools behind and starting to work with what is at hand out there.

My $\mathrm{PhD}$, then, is an attempt to increase the size of the conceptual box I inherited through education. It is an effort to illuminate the darkness and to see whether it is, indeed, populated by dragons. The main purpose of this dissertation is to emphasize that, while mysterious, there are no monsters in this so-called darkness. On the contrary, it is a place worth exploring, full of freedom, possibility and discovery. 


\section{TABLE OF CONTENTS}

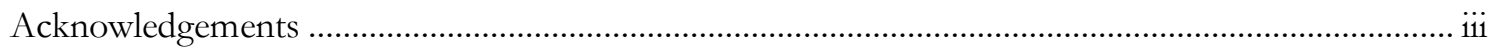

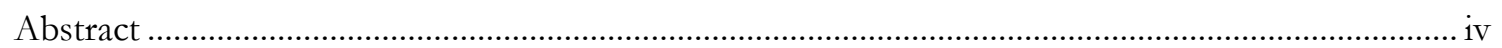

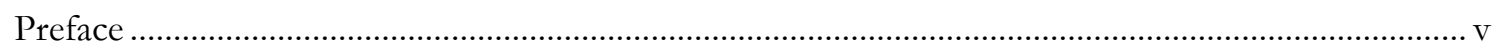

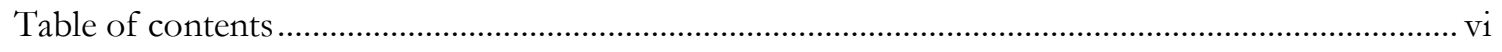

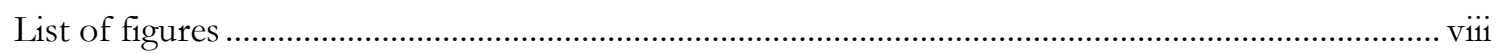

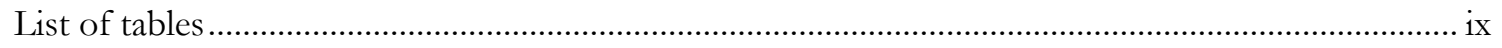

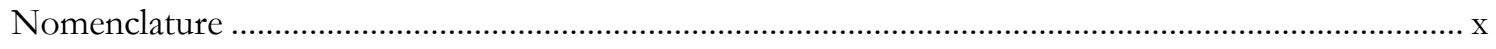

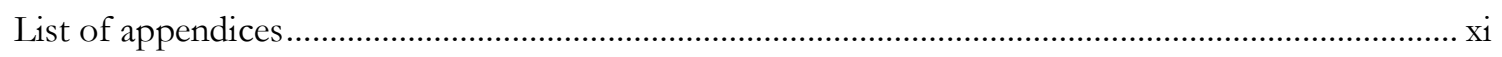

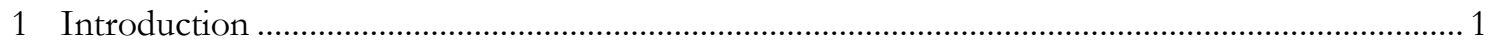

1.1 Research problem, research questions and contributions ............................................ 2

1.2 Justification for the research ….......................................................................................... 3

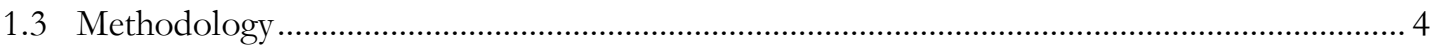

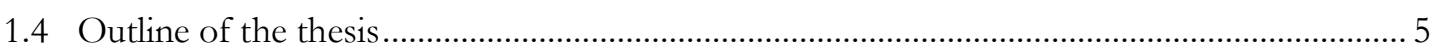

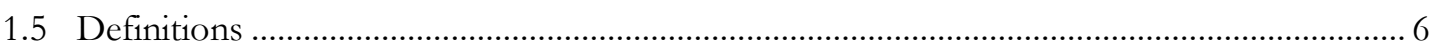

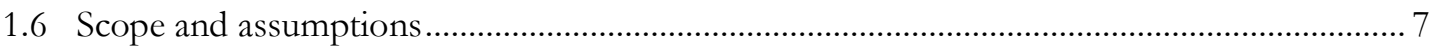

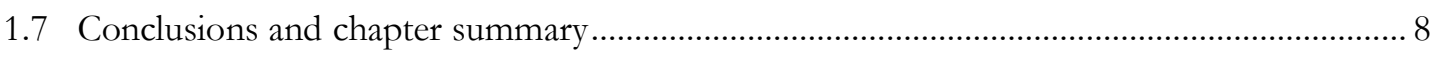

2 An unnecessary contradiction within building science's comfort research............................... 9

2.1 Comfort is defined as a state of the mind, but "the mind" has been traditionally ignored 10

2.2 Understanding how comfort develops with and without "the mind" ............................... 16

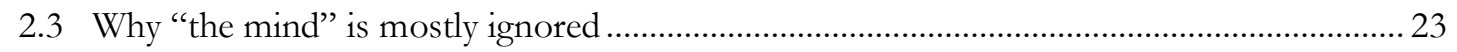

2.4 Embracing "the mind" through exploratory research ...................................................... 25

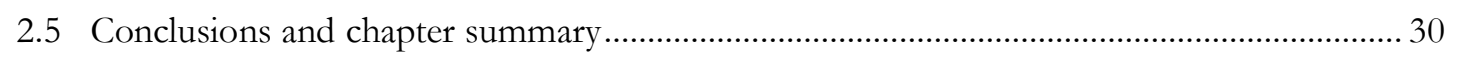

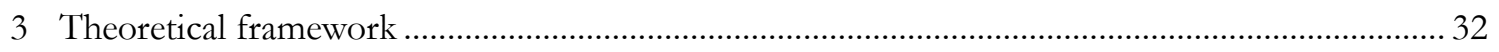

3.1 A framework for understanding how comfort develops ................................................... 33

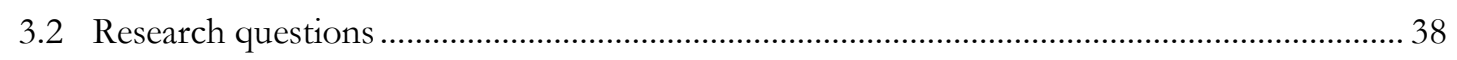

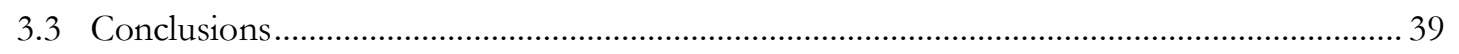

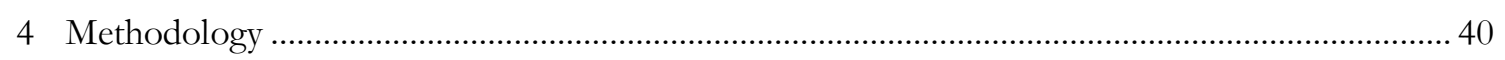

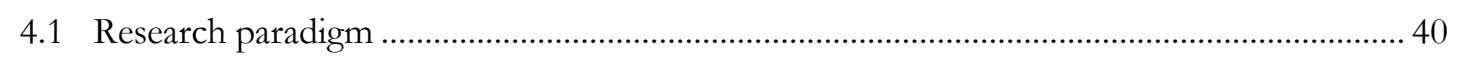

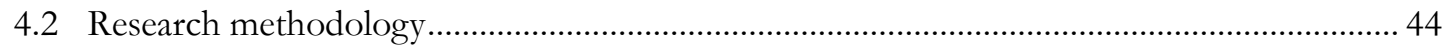

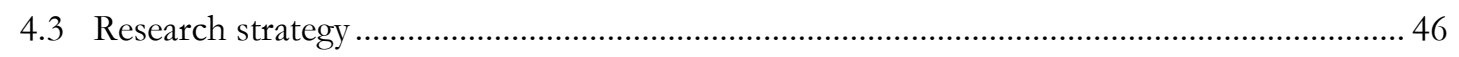

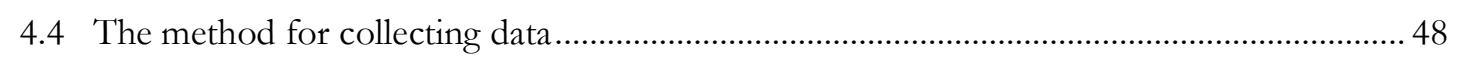

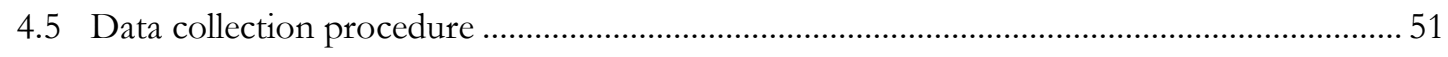

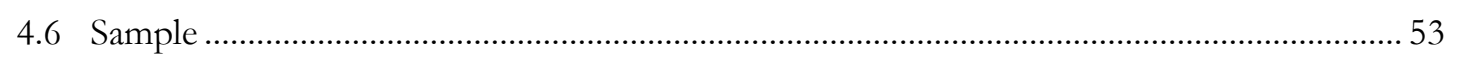

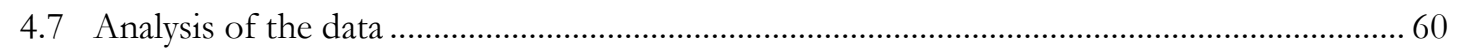

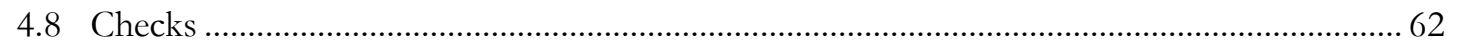

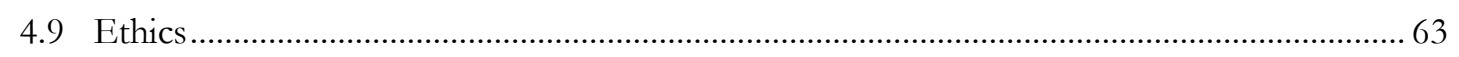

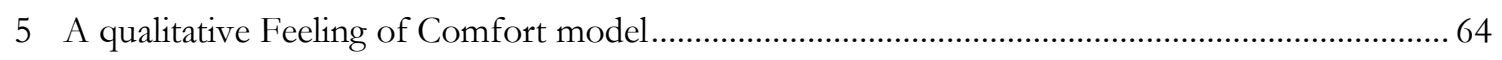

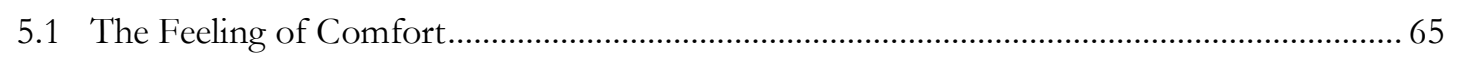

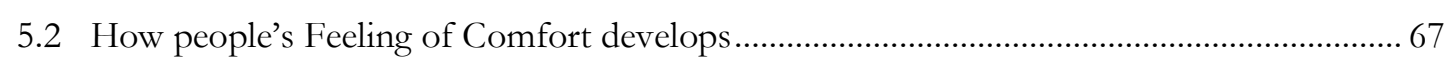




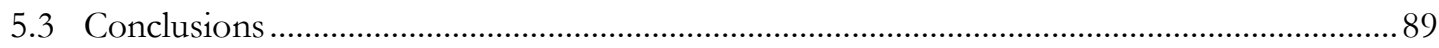

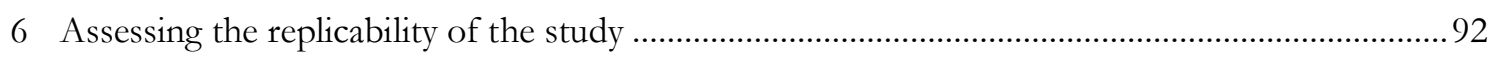

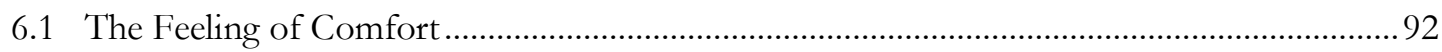

6.2 Making sense of new data with the Feeling of Comfort model .......................................... 95

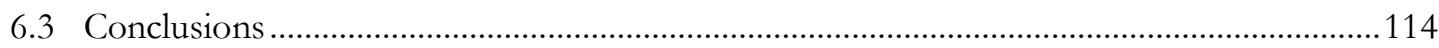

7 A mathematical model of the Feeling of Comfort in residential environments .......................115

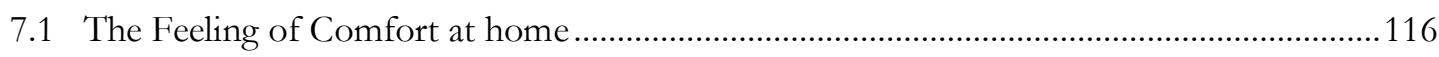

7.2 Modelling the immediate satisfaction with the room .........................................................118

7.3 Modelling the immediate satisfaction with the dwelling .................................................... 123

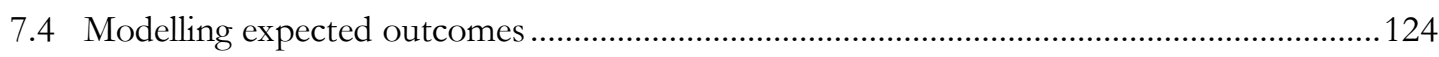

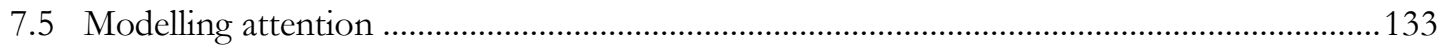

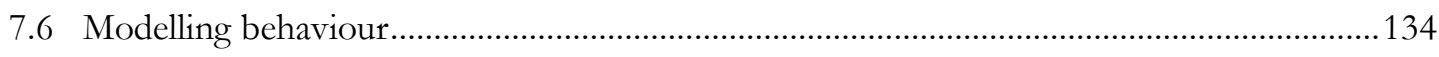

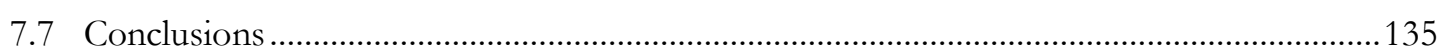

8 Towards a tool for simulating the Feeling of Comfort ….....................................................138

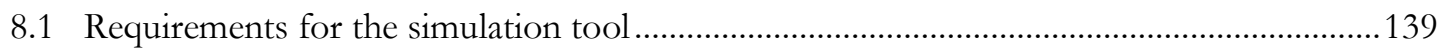

8.2 An architecture for simulating the Feeling of Comfort ......................................................141

8.3 Simulating people's Feeling of Comfort and behaviour .................................................144

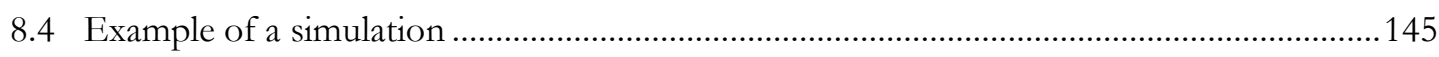

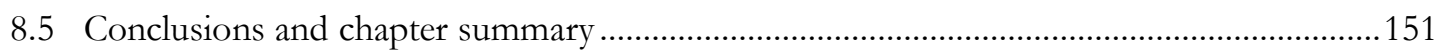

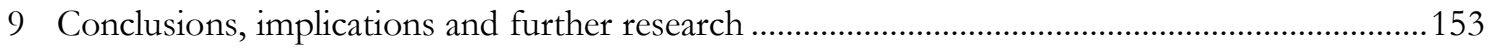

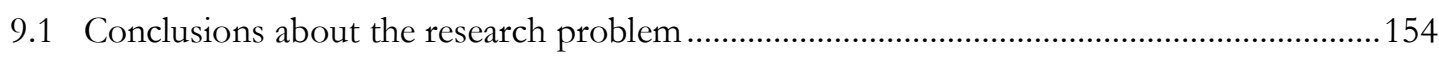

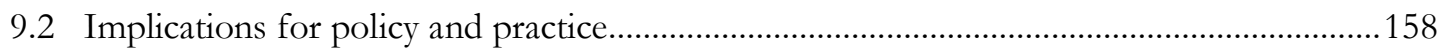

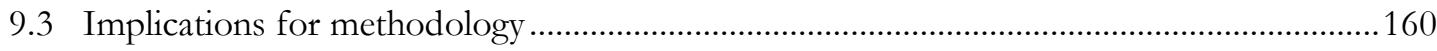

9.4 Limitations and opportunities for future research ............................................................ 162

9.5 Utilizing the qualitative Feeling of Comfort model in other contexts..............................165

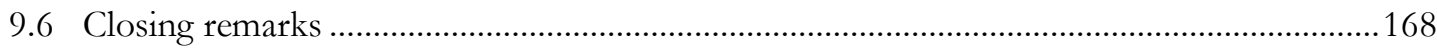

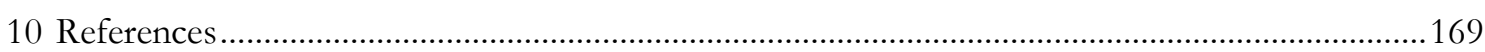

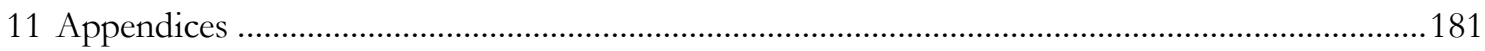




\section{LIST OF FIGURES}

Figure 1: Overview of the methodology utilized and of the structure of this thesis ............................ 5

Figure 2: How comfort develops according to the literature ................................................................... 34

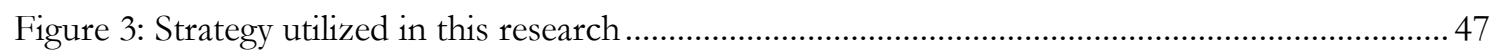

Figure 4: A small example of the mind map that resulted from an interview about air quality.......... 52

Figure 5: Cumulative number of codes related to daylight for each interview......................................58

Figure 6: Cumulative number of codes related to warmness for each interview ................................. 58

Figure 7: Cumulative number of codes related to coolness for each interview.....................................5 58

Figure 8: Cumulative number of codes related to acoustic performance for each interview.............. 59

Figure 9: Cumulative number of codes related to air quality for each interview.................................. 59

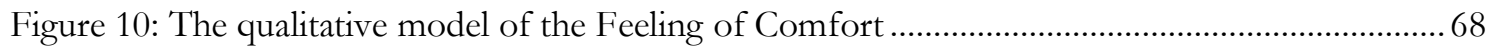

Figure 11: The Feeling of Comfort model adapted for its quantitative representation......................118

Figure 12: Impact of future over the present Feeling of Comfort, according to Equation 3 ..........126

Figure 13: Probability of a person attending to a situation according to Equation 8 ………….........134

Figure 14: Probability of engaging in a behaviour, according to Equation 9 ...................................135

Figure 15: Architecture of tool for simulating the Feeling of Comfort ............................................142

Figure 16: Paths of communication between the different modules...................................................143

Figure 17: Algorithm that allows for an efficient simulation of potential outcomes .........................144

Figure 18: Algorithm used for incorporating the Feeling of Comfort in building simulation ..........145

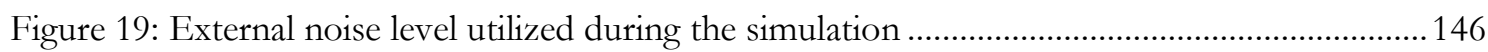

Figure 20: Floorplan of the dwelling modelled through the new simulation tool..............................147

Figure 21: Perceptions felt by the person living in the dwelling according to the simulation ..........149

Figure 22: How personal control affects the Feeling of Comfort according to the simulation........150

Figure 23: How busyness affects the Feeling of Comfort according to the simulation ....................151 


\section{LIST OF TABLES}

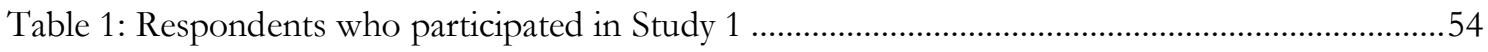

Table 2: Respondents who participated in Study 2 ............................................................................

Table 3: Objective indoor climatic variables identified for daylight and warmness ..............................70

Table 4: Environmental cues identified for daylight and warmness ....................................................72

Table 5: Internal elements identified for daylight and warmness..........................................................

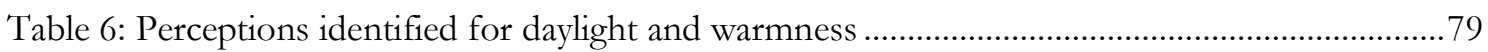

Table 7: Criteria used to infer expected outcomes identified for daylight and warmness ...................82

Table 8: Trade-offs identified for daylight and warmness .................................................................. 85

Table 9: Elements that define comfort for daylight and warmness ....................................................8

Table 10: Objective indoor climatic factors identified for acoustics, coolness and air quality...........96

Table 11: Environmental cues identified for acoustics, coolness and air quality.................................98

Table 12: Internal elements identified for acoustics, coolness and air quality ..................................100

Table 13: Perceptions identified for acoustics, coolness and air quality.............................................105

Table 14: Criteria used to infer expected outcomes for acoustics, coolness and air quality .............107

Table 15: Trade-offs identified for acoustics, coolness and air quality............................................109

Table 16: Elements that define comfort for acoustics, coolness and air quality ..............................111

Table 17: Perceptions that affect the immediate satisfaction with a room..........................................120

Table 18: How trade-offs can be incorporated into building performance simulation .....................122

Table 19: Number of potential future scenarios for different dwelling/occupant settings...............129

Table 20: Strategies and actions that allow improving comfort .......................................................132

Table 21: Relative importance of the different spaces .......................................................................147 
NOMENCLATURE

\begin{tabular}{|c|c|c|}
\hline Symbol & Meaning & Used in \\
\hline$s(\vec{m})$ & Immediate satisfaction with room $r$ & Section 7.2 \\
\hline$\vec{m}$ & $\begin{array}{l}\text { Perceptions and the factors outside of the realm of building science that are } \\
\text { relevant for people's wellbeing }\end{array}$ & Section 7.2 .1 \\
\hline$\vec{q}$ & $\begin{array}{l}\text { set of quantifiable elements of the physical world from which the elements } \\
\text { in } \vec{m} \text { can be estimated }\end{array}$ & Section 7.2 .1 \\
\hline$\sigma$ & Immediate satisfaction with the dwelling as a whole & $\begin{array}{l}\text { Equation } 1 \\
\text { Section } 7.3\end{array}$ \\
\hline$p_{r}$ & $\begin{array}{l}\text { Subjective importance of room } r \text { in the determination of the immediate } \\
\text { satisfaction with the dwelling at a specific moment of time }\end{array}$ & $\begin{array}{l}\text { Equation } 1 \\
\text { Section } 7.3\end{array}$ \\
\hline$\kappa$ & $\begin{array}{l}\text { The Feeling of Comfort, considering expected outcomes but not systems' } \\
\text { reliability }\end{array}$ & $\begin{array}{l}\text { Equation } 2 \\
\text { Section } 7.4\end{array}$ \\
\hline$\varphi$ & The impact of the future on people's current state of mind & $\begin{array}{l}\text { Equation 3, } \\
\text { Section } 7.4\end{array}$ \\
\hline$h_{1}, h_{2}$ & $\begin{array}{l}\text { The time into the future after which the impact of future perceptions over } \\
\text { people's current comfort start to decay and finish decaying, respectively }\end{array}$ & $\begin{array}{l}\text { Equation } 3 \\
\text { Section } 7.4\end{array}$ \\
\hline $\mathrm{K}$ & $\begin{array}{l}\text { The Feeling of Comfort, considering expected outcomes (Equation 2) and } \\
\text { systems' reliability }\end{array}$ & $\begin{array}{l}\text { Equation } 4 \\
\text { Section } 7.4 .1\end{array}$ \\
\hline$\kappa_{f}, \kappa_{n f}$ & $\begin{array}{l}\text { The feeling of comfort in cases of system's failure and non-failure, } \\
\text { respectively }\end{array}$ & $\begin{array}{l}\text { Equation } 4 \\
\text { Section } 7.4 .1\end{array}$ \\
\hline$f_{p}$ & The perceived probability of system failure & $\begin{array}{l}\text { Equation } 4, \\
\text { Section } 7.4 .1\end{array}$ \\
\hline $\mathrm{K}_{\text {best }}$ & $\begin{array}{l}\text { The Feeling of Comfort associated with the best-expected outcome inferred } \\
\text { by the subject who is developing the Feeling of Comfort }\end{array}$ & Section 7.4 .2 \\
\hline$\alpha$ & Probability of a person attending to a situation & $\begin{array}{l}\text { Equation } 8 \\
\text { Section } 7.5\end{array}$ \\
\hline$\beta$ & An indicator of how busy a person is & $\begin{array}{l}\text { Equation } 8 \\
\text { Section } 7.5\end{array}$ \\
\hline $\mathrm{B}$ & The probability of a person taking action to improve comfort & $\begin{array}{l}\text { Equation 9, } \\
\text { Section } 7.6\end{array}$ \\
\hline$\pi$ & An indicator of the proactivity of the person & $\begin{array}{l}\text { Equation 9, } \\
\text { Section } 7.6\end{array}$ \\
\hline$\varepsilon$ & $\begin{array}{l}\text { Small number utilized to represent a negligible amount (a value of } 0.001 \\
\text { was used in this dissertation) }\end{array}$ & $\begin{array}{l}\text { Equations } 3,8 \\
\text { and } 9\end{array}$ \\
\hline$T S$ & Thermal Sensation - Perception of how cold or hot a space is & Equation 10 \\
\hline$L$ & Loudness - Perception of how quiet or loud a space is & Equation 10 \\
\hline$B r$ & Brightness - Perception of how dark or bright a space is & Equation 10 \\
\hline$U B$ & Utility Bills - Perception of Utility Bills being too high or too low & Equation 10 \\
\hline Clo & Clothing annoyance - Perception of wearing too much or too little clothing & Equation 10 \\
\hline
\end{tabular}




\section{LIST OF APPENDICES}

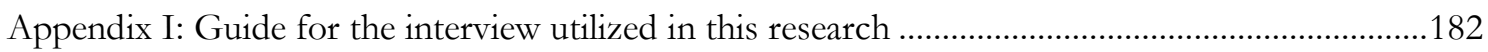

Appendix II: Respondent information sheet, in English and Spanish .............................................187

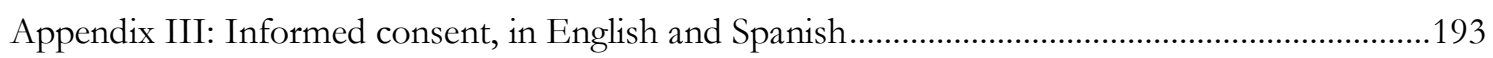

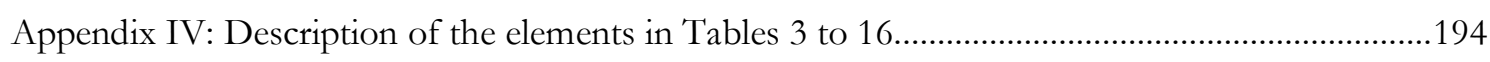

Appendix V: Overview of the routines utilized for the simulation in Section 8.4 ............................194 



\section{INTRODUCTION}

"In a bole in the ground there lived a hobbit. Not a nasty, dirty, wet hole, filled with the ends of worms and an oozy smell, nor yet a dry, bare, sandy bole with nothing in it to sit down on or to eat: it was a hobbit-hole, and that means comfort"

JRR TOLKIEN, THE HOBBIT

Providing comfort is one of the most important objectives of dwellings (Blanchett, 2017; Rodriguez \& Siret, 2009). There are three reasons for this. First, because the lack of comfort affects people's lives at various levels, from harmless nuisances (Cutler, Sheng, Martin, Glaser, \& Andersen, 2008; Karmann, Schiavon, \& Bauman, 2017; Tzempelikos, Athienitis, \& Karava, 2007; Tzempelikos, Bessoudo, Athienitis, \& Zmeureanu, 2010) to more severe health issues (Howden-Chapman et al., 2005, 2007). Second, because a significant portion of the energy consumption in the housing sector is directly associated with people seeking to feel more comfortable within their dwellings (Isaacs et al., 2006; Isaacs, Saville-Smith, Camilleri, \& Burrough, 2010). And third, but not less important, because comfort-utilized in broad terms - seems to be part of what people expect to find in their dwellings. For instance, people tend to associate the action of purchasing a dwelling with a higher quality of living (Koklic \& Vida, 2009), increased comfort (Dunning, 2016, p. 127), and an improved housing situation (Clark, 1993).

However, comfort is an elusive term and thus it is common practice to reduce its complexity at the moment of operationalizing it (Altomonte et al., 2020). Specifically, the discipline of building science-which is supposed to be the main audience of this dissertation—-tends to utilize the more precise term indoor environmental quality (Dorizas, De Groote, \& Volt, 2018; Mujan, Anđelković, Munćan, Kljajić, \& Ružić, 2019; Nimlyat, 2018; Sarbu \& Sebarchievici, 2013). This concept focuses exclusively on visual, acoustic, and thermal comfort, and indoor air quality (Villeneuve \& O'Brien, 2020). This research studies the differences between the broader concept of comfort and its simplified version, indoor environmental quality, from a building science perspective. It concludes that it is not only necessary to reduce the differences between these concepts but that doing so is possible. 


\subsection{Research problem, research questions and contributions}

The building science literature often introduces the concept of comfort as "a subjective state of the mind" (Altomonte et al., 2020). In spite of that, building science's comfort research and practice tend to focus almost exclusively on objective environmental factors and on perceptions that can be directly associated with people's physiological system (e.g., ASHRAE, 1999; Fanger, 1989; Fountain \& Huizenga, 1996; Jakubiec \& Reinhart, 2012; Sarey Khanie, Stoll, Einhäuser, Wienold, \& Andersen, 2017; van Hoof, Mazej, \& Hensen, 2010; Wienold \& Christoffersen, 2006; Wienold et al., 2019). This is a contradiction because, while the concept of comfort is initially defined as subjective, comfort research and practice tend to avoid dealing with the complexity of the subject by focusing almost exclusively on physical and physiological elements. Likewise, while comfort is said to be a state of the mind, the cognitive processes that characterize the mind have virtually never been considered. These often-disregarded factors-i.e., people's nonphysical personal factors and their cognitive processes-are what this research often refers to as "the mind" 1 .

It is important to acknowledge, however, that this contradiction is not rooted in ignorance but pragmatism. That is to say, the reason building science tends to disregard "the mind" is to avoid the overwhelming complexity that it can introduce into both comfort research and practice. According to Reinhart (2014, p. 27), researchers "fear that a quality assessment of e.g., the 'visual interest of a space' leaves too much room for subjective interpretation". This is evident in the ASHRAE standard 62 (1999) which focuses exclusively on the presence of indoor pollutants while simultaneously stating that many other factors affect people's satisfaction with the air inside of buildings. Likewise, the reason to reduce thermal comfort to physical/physiological parameters is that "satisfaction with the thermal environment is a complex subjective response to several interacting and less tangible variables. In other words, there is really no absolute standard for thermal comfort. [However] in general comfort occurs when... physiological regulation is minimized" (Djongyang, Tchinda, \& Njomo, 2010, p. 2627). In other words, building scientists seem to avoid incorporating "the mind" to prevent their frameworks, models and theories from being overwhelmingly complex.

This research aims to mitigate this contradiction by exploring "the mind" in the context of people's comfort and by proposing ways in which it can be better incorporated into building science's research and practice. This overall goal can be

\footnotetext{
${ }^{1}$ Section 1.5 provides a more comprehensive definition of what this dissertation calls "the mind"
} 
broken down into three research questions:

1. What are the main non-physical personal factors and cognitive processes that affect people's comfort?

2. What is the role of the non-physical personal factors and the cognitive processes in the development of people's comfort?

3. How can predictive models of comfort incorporate people's non-physical characteristics and their cognitive processes?

This research answers the first two questions by developing a new qualitative model of the Feeling of Comfort (Chapters 5 and 6). This model can serve as a framework for guiding comfort research and practice. For instance, for developing a quantitative model of comfort (Chapter 7 shows an example of this) which can later be incorporated into building performance simulation tools capable of evaluating the Feeling of Comfort of different dwelling design alternatives (Chapter 8 shows an example of this).

This research not only suggests that embracing "the mind" is crucial for properly understanding people's feeling of comfort but also evidences that doing so is possible. The latter is its major contribution to building science. However, this research also suggests that incorporating "the mind" into building science's research and practice requires developing new tools and conceptual frameworks as well as using research methods that historically have not been part of the building science discipline.

\subsection{Justification for the research}

As explained earlier, building science seems to disregard "the mind" to avoid the overwhelming complexity it might introduce to the discipline. While reasonable from a practical perspective, this decision is not supported by the literature because "the mind" has not been shown to play an insignificant role in the determination of people's comfort. On the contrary, evidence suggests that elements more related to "the mind" than to physics and physiology do influence people's comfort. An example of this is that people who have control over their environment seem to evaluate spaces differently from those who do not (Luo et al., 2014; Veitch, 2001). Likewise, people's perception of the indoor environment changes depending on their expectations (Luo et al., 2016; Schweiker, Rissetto, \& Wagner, 2020) and on how a space looks and what they believe its temperature to be (Rohles, 2007).

The value of this research, then, resides in the fact that it attempts to understand the role of "the mind" in the determination of people's comfort. Additionally, this research is 
valuable because, after concluding that the role of "the mind" is relatively important, it proposes a means of incorporating it into building science's research and practice.

\subsection{Methodology}

This research aims to explore the "the mind" in the context of people's comfort and to propose a way in which it can be better incorporated into building science's research and practice. Consequently, its first step (Chapter 5) was an exploration of people's understanding of comfort in which 18 volunteers-from Chile and New Zealand and without theoretical knowledge about comfort-were asked to describe comfortable homes. Specifically, they were asked to describe "a home with good daylight" and "a warm home". The reason to interview people with no proficiency in this topic was to understand what non-experts-i.e., the people whose homes building science is supposed to make more comfortable-expect from and mean by "a comfortable home". (The point of view of experts was gathered through a literature review.) The data gathered during this stage made it possible to develop a qualitative model of comfort: a model of the Feeling of Comfort.

Later, a second study (Chapter 6) helped in the assessment of whether the Feeling of Comfort model was able to make sense of data gathered by interviewing different people and associated with different indoor environmental quality domains. The model was successful in making sense of people's descriptions of "cool homes", "homes with good acoustic performance", and "homes with good air quality". People from Chile and New Zealand-24 respondents in total-participated in this second study.

After developing a qualitative model of the Feeling of Comfort, this research aimed to assess whether "the mind" — understood through said model—could be incorporated into the quantitative practices of building science. This was proved possible through an example. That is, by developing a mathematical representation of the model and by performing a building performance simulation based on this quantitative model.

Overall, this process (shown in Figure 1) answered the three proposed research questions and concluded that incorporating "the mind" into building science research and practice is not only possible but crucial. However, it led to the realization that doing so requires acquiring or developing new conceptual frameworks, research methods and tools. 


\subsection{Outline of the thesis}

Apart from the present chapter, the structure of this dissertation-only partially shown in Figure 1-is as follows.

Chapter 2 contains a literature review that concludes that while building science introduces comfort as a subjective state of the mind, its research and practice tend to disregard "the mind" as much as possible. This review covers mostly building science literature but also draws a significant amount of knowledge from other disciplines.

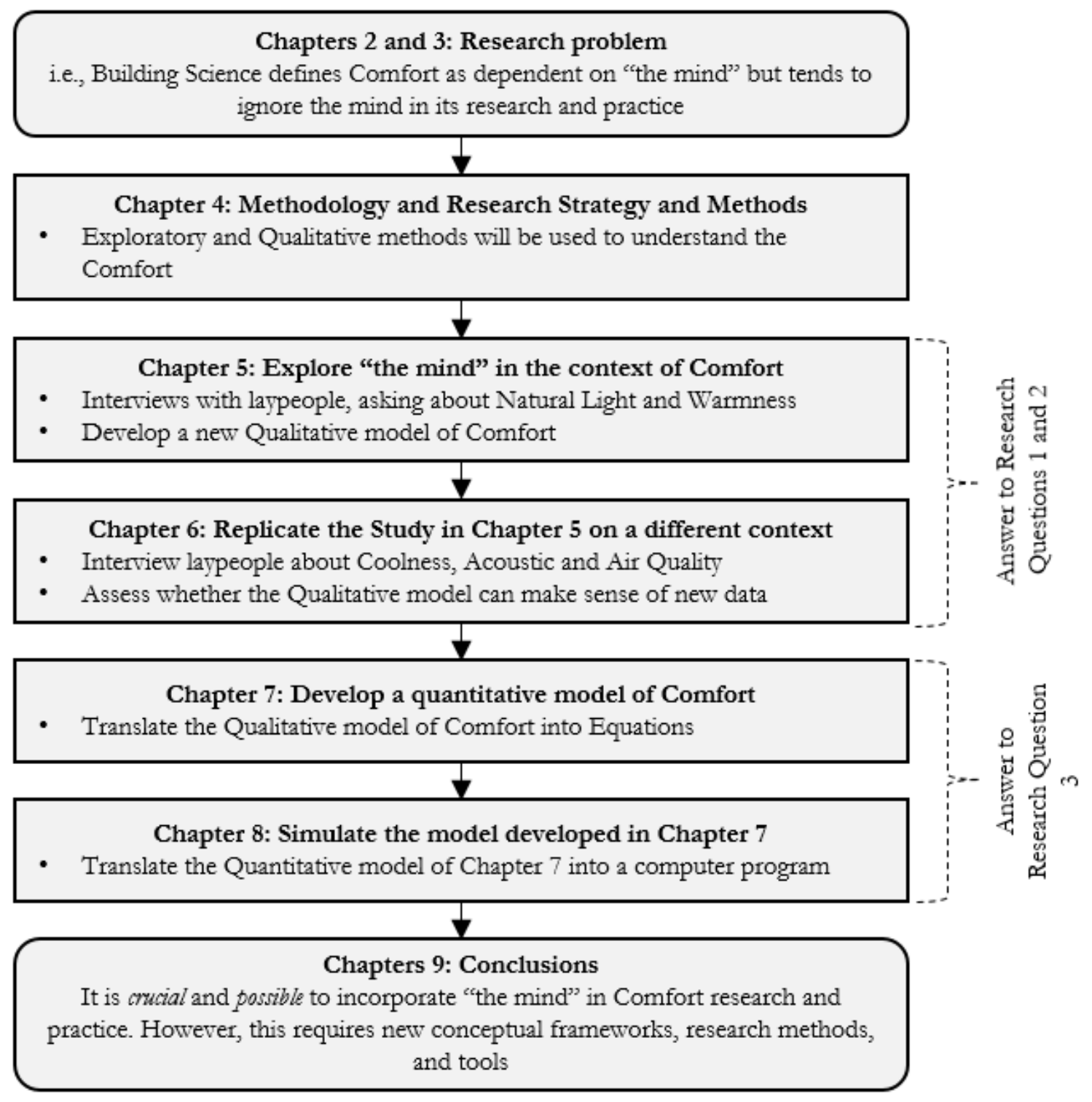

Figure 1: Overview of the methodology utilized and of the structure of this thesis

After identifying the research problem, Chapter 3 develops a theoretical framework that can help understand how people's comfort develops, according to the literature. Three research questions were derived from this framework. They are answered in later chapters.

Chapter 4 introduces the methodology utilized in this research by explaining and 
justifying the research paradigm from which this research was performed and the methods utilized for gathering and analysing data. It also presents the sample used for each study.

Chapter 5 discusses the results of Study 1 . That is to say, it introduces a qualitative model of the Feeling of Comfort that will drive the rest of this dissertation. Chapter 6 then discusses the results of Study 2, which successfully replicates Study 1 by gathering data from different sources (i.e., interviewing different people) and focuses on a different set of indoor environmental quality domains. Chapter 7 introduces a quantitative representation of the Feeling of Comfort (i.e., of the model developed in Chapter 5). This quantitative model then informs Chapter 8, which demonstrates how the Feeling of

Comfort could be introduced into building performance simulation tools by presenting one way of doing this.

Finally, Chapter 9 presents the overall conclusions of this research by revisiting the research problem identified in Chapter 2, although this time considering the new insights contributed by this research. This chapter also outlines the implications and opportunities for future research that arise from the results of this research.

\subsection{Definitions}

This dissertation utilizes some specific terms whose meaning varies between disciplines, and/or that some disciplines consider vague. To avoid misunderstanding, this section defines said concepts.

The first term worth defining was already introduced in this chapter; i.e., "the mind". Within this dissertation, "the mind" is an instrumental umbrella term that groups together people's cognition and their non-physical personal factors (e.g., household composition, budget, and lifestyle). In other words, the two elements that-according to this dissertation-building science's comfort research and practice have often disregarded. It is worth mentioning that people's internal elements include all the characteristics of people that cannot be addressed through physiology or physics. This includes those relatively stable characteristics (e.g., household composition, lifestyle, willingness to pay for utility bills) and also the highly situational ones (e.g., the amount of time that a person is planning to stay on a certain place, or if the person is currently responsible for the safety of someone else in the dwelling). Also, because this dissertation considers Comfort to be a great motivator to behaviour, this thesis attempts to understand "the mind" mostly from a psychological/behavioural science approach (as opposed to, for instance, phenomenology, which would understand Comfort to be an experience). 
Intuition, on its part, is one of the two human reasoning systems (Stanovich \& West, 2000). Intuition is a reasoning system that can only be trusted in some circumstances (Kahneman \& Klein, 2009) but that has the advantage of being quick, mostly automatic, largely unconscious, and requires low cognitive effort (Kahneman, 2003; Stanovich \& West, 2000). The other system, reason, relates to the analytic intelligence that has most traditionally been studied (Kahneman, 2003; Stanovich \& West, 2000). The most formal names for intuition and reasoning are System 1 and System 2 (Evans, 2003; Stanovich \& West, 2000). Nevertheless, they are also known as automatic and reflective systems (Thaler \& Sunstein, 2008) and by other names (Evans, 2003, 2008). Because they are easy to remember and intuitive to understand, this dissertation utilizes the terms of Intuition and Reasoning, as used by Kahneman (2003).

Finally, it is worth defining the term feeling, which characterizes the qualitative model proposed by this research (developed and tested in chapters 5 and 6, respectively). Within this dissertation, the term feeling is associated with emotions. It attempts to emphasize thatcontrary to the traditional conceptualization of comfort used by building science (see chapters 2 and 3) - the Feeling of Comfort model does not directly link people's satisfaction with the environment to physiological phenomena. That is to say, contrary to glare (Jakubiec \& Reinhart, 2012; Konstantzos \& Tzempelikos, 2017; Sarey Khanie et al., 2017; Sawicki \& Wolska, 2015; Wienold, 2009; Wienold \& Christoffersen, 2006), thermal sensation (ASHRAE, 2010; ISO, 1984), and loudness (Cowan, 1994; Doelle, 1972; Egan, 1988), which can be associated to people's eyes, skin and ears, respectively, the Feeling of Comfort is located in "the mind". For example, the Feeling of Comfort model is not too concerned with whether people are feeling hot or cold (which might be seen as a purely physiological sensation). Similarly, it does not ask whether people like feeling cold or hot at this particular moment (which includes a more affective perception). On the contrary, the Feeling of Comfort model attempts to understand whether, considering the current situation, people care about a room being too hot or too cold. This is what distinguishes the Feeling of Comfort from purely physiological responses.

\subsection{Scope and assumptions}

Due to practical and disciplinary reasons, this research dwells within a specific scope and thus its results should be interpreted within such a scope.

For instance, the first — and perhaps the most noticeable— limitation of the study relates to the fact that the data is acquired by interviewing people who recently intended to purchase a residence in Chile or New Zealand. Since people who intend to purchase 
homes probably have an economic status that is not representative of the whole population of Chile or New Zealand, applying the results of this research in other socio-cultural contexts requires some consideration. This limitation, however, is mitigated in two ways. First, the chances that the results of this dissertation are highly dependent on culture are reduced by performing research in two different countries/cultures (i.e., Chile and New Zealand). And second, the chances that these results are highly dependent on the comfort domain being studied are also reduced by performing a replication study (Chapter 6).

It is also important to notice that this research focuses on people's own dwellings and thus its results might not be valid in situations where people are visitors (e.g., a friend's home, hotels) or for other building typologies (e.g., commercial, educational).

On the other hand, it is worth mentioning that this is building science research and thus the concept of comfort that this dissertation introduces is very much built around indoor environmental quality. In other words, even this dissertation argues that comfort has to do with wellbeing and freedom and is determined by non-environmental aspectssuch as safety and financial concerns-generalizing the results of this research to broader comfort connotations requires careful consideration.

\subsection{Conclusions and chapter summary}

This chapter provided the basis for this dissertation, introducing the research problem, its aims, and research questions. This chapter also described the potential significance of the present research, defined key terms, and briefly described and justified the methodology. Then, it explained the structure of this dissertation and discussed the scope of the research and its results. 


\title{
2 AN UNNECESSARY CONTRADICTION WITHIN BUILDING SCIENCE'S COMFORT RESEARCH
}

\author{
"The stone was cold beneath her feet, and the wind was blowing fiercely, as it always did up \\ here, but the view made her forget all that for half a beartbeat"
}

GEORgE R. R. MARTIN, A GAME OF THRONES

Within building science, the concept of comfort is mostly introduced as a subjective state of the mind (e.g., Auliciems, 1981, 1983; de Dear et al., 2016). Despite this, the models that building science uses for assessing comfort tend to depend mostly on objective environmental factors and on perceptions that can be directly associated with people's physiological system (e.g., Fountain \& Huizenga, 1996; Jakubiec \& Reinhart, 2012; Sarey Khanie, Stoll, Einhäuser, Wienold, \& Andersen, 2017; van Hoof, Mazej, \& Hensen, 2010; Wienold \& Christoffersen, 2006; Wienold et al., 2019). That is to say, while comfort is defined as subjective, models of comfort avoid dealing with the complexity of the subject by only focusing on physical personal factors (e.g., ASHRAE, 2010). Similarly, while comfort is considered to be a state of the mind, most models ignore cognition as much as possible by directly associating comfort with sensory perceptions that can be calculated from objective environmental variables only (Jakubiec \& Reinhart, 2012; Wienold \& Christoffersen, 2006). This is a contradiction. Either comfort is a subjective state of the mind or it can be calculated from objective (physical) parameters only. This dissertation will refer to these often-ignored elements-i.e., people's cognitive processes and relevant non-physical personal factors that affect people's comfort—as “the mind".

Despite being often disregarded, "the mind" is known to affect people's perception of the indoor environment. For instance, evidence suggests that people who have control over their environment might evaluate spaces differently from those who do not (Luo et al., 2014; Veitch, 2001). Likewise, people's perception of the indoor environment changes depending on their expectations (Luo et al., 2016; Schweiker, Rissetto, et al., 2020) and the appearance of a space (Rohles, 2007). Thus, this research is an effort to incorporate "the mind" into 
building science's comfort research and practice, mitigating the contradiction mentioned earlier.

This chapter contains four main sections. First, it provides more details about the contradiction that exists in building science's literature on comfort. Specifically, it states that the de-facto definition of comfort tries to ignore "the mind" as much as possible. The second section outlines the process through which comfort develops according to the literature, concluding that building science has managed to ignore "the mind" by too strongly associating comfort with physiology and physics. The third section argues that the reason to ignore "the mind" is not rooted in ignorance but pragmatism. That is to say, ignoring "the mind" is a choice that has the purpose of making the topic of comfort something more manageable by building science's tools and methods. Finally, the fourth section argues that models of comfort can embrace "the mind" without becoming unusably complex. In other words, this oversimplification of comfort—which leads to the contradiction raised earlieris not strictly necessary.

\subsection{Comfort is defined as a state of the mind, but "the mind" has been traditionally ignored}

The fact that comfort is a state of the mind has been, for a long time, well established in building science. Back in 1967, Gagge, Stolwijk, and Hardy (1967, p. 1) argued that “"comfort' is a recognizable state of feeling, but possesses no identifiable sense organ like the basic five senses". Nowadays, phrases like "comfort is in the mind of the beholder" (de Dear et al., 2016) and definitions of comfort in the form of "condition of the mind that expresses satisfaction with the environment" are easily found in the literature (Altomonte et al., 2020). For example, Fabbri (2013, p. 3) argues that "the difficulty lies in the evaluation of thermal comfort, because it is a judgment that depends not only on the energy exchanges with the body-environment, but also includes the psychological, emotional, cultural and social aspects of people". Similarly, Hensen (1991, p. 2.4) explains that thermal comfort "is an emotional experience which can be characterized in terms of 'pleasant' or 'unpleasant"'.

Even though it is widely accepted, this paradigm - i.e., comfort as a state of the minddoes not seem to influence more than a few paragraphs of any building science book, dissertation, or journal article. For instance, in her Indoor Environment Handbook, Bluyssen (2009, p. 4) posits that "aesthetic quality and spatial and ergonomic quality are also part of the indoor environment". However, she almost immediately adds that her book only focuses on environmental parameters. So, even if building scientists know that comfort is a state of the mind, models for assessing it generally bypass "the mind" by making it a more concrete 
and objective concept. For instance, by developing equations that depend only on physical quantities in the environment (e.g., ASHRAE, 2010; Bean, 2020; Wienold \& Christoffersen, 2006). In the end, the indoor environment is often described through environmental stressors alone (Bluyssen, 2009, p. 4). This seems to happen in the four main elements of indoor environmental quality; that is to say, thermal, visual and acoustic comfort, and air quality (Andargie, Touchie, \& O’Brien, 2019).

Within the thermal domain, definitions of comfort often emphasize subjectivity and it is often clearly stated that thermal sensation-i.e., the fact that a room feels warm, hot, cool or cold- "says nothing about whether the occupants are actually going to like it" (de Dear, 2011, p. 111). Despite this, thermal comfort tends to very quickly be equated to the concept of thermal sensation, which can be estimated from objective quantities alone. In fact, de Dear and Brager (2002, p. 549) seem to share this concern when stating that "it is commonly agreed within the thermal comfort research community that 'acceptable' is synonymous with 'satisfaction', and that 'satisfaction' is associated with thermal sensations of 'slightly warm', 'neutral', and 'slightly cool'”. For instance, Lawrence Race presents both a complex and a simplified definition of comfort in the same sentence when stating that "thermal comfort [exists when] there is broad satisfaction with the thermal environment i.e., most people feel neither too hot nor too cold" (2006, p. 4). That is to say, thermal comfort initially reflects satisfaction - a concept that holds a highly subjective connotation-but quickly falls into the much more simplified thermal sensation. Likewise, Auliciems and Szokolay argue that "physiological neutrality... does not necessarily mean comfort" (1997, p. 16) while also suggesting that thermal comfort is the "minimization of physiological responses" (1997, p. 3). Fabbri, on the other hand, argues that comfort is difficult to evaluate because "it is a judgment that depends not only on the energy exchanges with the bodyenvironment" (2013, p. 3). Yet, Fabbri also mentions that "comfort can be expressed with indices and temperature sensation" (2013, p. 75). Similarly, Bean argues that "the roots of thermal comfort are based upon physiology and equally as important—psychology; that is how do people perceive the thermal environment" (2020, p. 7). However, in the end, the only personal factors that he mentions to affect thermal comfort are those physical personal factors affecting thermal sensation: clothing and metabolic rate (i.e., those in the ASHRAE $55,2010)$. This pattern - in which comfort is simplified to a nearly-objective sensation-is present in both major paradigms of thermal comfort research. That is to say, in the rational approach to thermal comfort (Djongyang et al., 2010) and the adaptive one (Brager \& de Dear, 1998; Humphreys, Nicol, \& Roaf, 2016; Nicol, Humphreys, \& Roaf, 2012).

In the case of research developed through the rational approach, thermal comfort is said 
to depend only on thermodynamically relevant factors like temperature, wind speed, relative humidity, and people's clothing and metabolic rate (ASHRAE, 2010; ISO, 1984; Olesen, 2000). In other words, the rational approach to thermal comfort tends to unambiguously exclude "the mind", even if some exceptions do exist. For example, Fanger and Toftum (2002) added an expectancy factor to their model, and Yao, Li and Liu (2009) included expectations, culture and previous experiences in their predictions. Still, both these studies equate comfort to a neutral thermal sensation, thus contradicting the building science's literature referenced earlier in this same section.

Research performed from the adaptive approach to thermal comfort, for its part, leaves some room for "the mind" by acknowledging that people engage in behavioural, physiological and psychological adaptation (Brager \& de Dear, 1998; Humphreys et al., 2016; Nicol et al., 2012). Nevertheless, studies on adaptive comfort research still mostly focus on developing regressions between thermal sensation votes and environmental parameters, looking to identify the temperature that feels neutral (Parkinson, de Dear, \& Brager, 2020, p. 6). In other words, while adaptive thermal comfort models do incorporate non-physical elements of the individual (i.e., some of "the mind"), they still estimate people's comfort from objective environmental variables only and equate comfort to thermal sensation.

The contradiction raised in this section is very often present in more or less explicit ways in an immense number of studies (e.g., Carlucci and Pagliano; 2012 review more than 80 thermal discomfort indices developed up to 2010). So, for the sake of briefness, what remains of this section will focus only on some of the most influential guidelines of thermal comfort.

The ASHRAE Standard 55 (2010, p. 3) defines thermal comfort as "that condition of the mind that expresses satisfaction with the thermal environment and is assessed by subjective evaluation". On the same page, however, it removes "the mind" by defining the thermal environment as "the characteristics of the environment that affect a person's heat loss". The old ISO7730 (1984, p. 4) standard defines thermal comfort in the same way as the ASHRAE 55 (2010, p. 3). However, right after providing such a definition, it simplifies the concept by stating that "dissatisfaction may be caused by warm or cool discomfort for the body as a whole as expressed by the PMV and PPD-indices" (ISO, 1984, p. 4). The PPDindex predicts "the percentage of people likely to feel too hot or too cold" (ISO, 1984, p. 1); which means that it is directly linked to thermal sensation and thus it is "mainly related to the thermal balance of [people's] body as a whole" (1984, p. 1).

It is worth noticing, however, that the "method for determining acceptable thermal conditions in naturally conditioned spaces" presented in the ASHRAE Standard 55 (2010, p. 
11) does not rely on people's body heat (im)balances (i.e., thermal sensations) but on their preferences (de Dear \& Brager, 2002). Instead of looking for the temperature where people feel neither hot nor cold, it intends to determine the temperature at which people do not want the space to be colder or warmer. This certainly represents a great step towards the incorporation of "the mind" in comfort research; however, this model still strongly and exclusively associates comfort with purely objective environmental factors.

The daylight domain understands that the quality of daylight is not limited to the effects of light in people's eyes. On the contrary, building scientists understand that daylight can psychologically and aesthetically affect how people perceive spaces (Chamilothori et al., 2019; Chinazzo, Wienold, \& Andersen, 2018b, 2018a). For instance, daylight is known to improve health and wellbeing (Jakubiec \& Reinhart, 2012, p. 149) and it is understood that its quality relates to both qualitative and quantitative factors (Andersen, Guillemin, Amundadottir, \& Rockcastle, 2013). Nevertheless, daylight quality and visual comfort are often separated. For instance, even if direct sunlight is often considered an enemy of visual comfort because of its excessive power (IESNA, 2012), the contrast it creates has been associated with daylight quality because of its positive perceptual effects (Rockcastle, Amundadottir, \& Andersen, 2017; Rockcastle \& Andersen, 2012, 2013, 2014).

The difference between daylight quality and visual comfort seems to be that, while the former is subjective, qualitative, complex, and located in "the mind"; the latter is simpler, more objective, and located in people's eyes. Moreover, contrary to the case of daylight quality, building scientists assume that visual comfort can be estimated from objective environmental parameters alone. For instance, visual comfort is often strongly associated with glare (Jakubiec \& Reinhart, 2015); that is to say, with the unpleasant physiological sensation caused by excessive light or contrast (Jakubiec \& Reinhart, 2012).

Ignoring the perceptual and aesthetic aspects of daylight and focusing on the more objective Visual Comfort is analogous to equating thermal comfort to thermal sensation. In the end, subjectivity is avoided and comfort is assumed to exist when people's physiological regulatory processes are minimized. Based on this principle, daylight studies mostly aim to provide enough light to be able to operate (e.g., work, read, cook) while simultaneously avoiding glare and/or overheating (e.g., IESNA, 2012; Nabil \& Mardaljevic, 2005, 2006; Pierson, Wienold, \& Bodart, 2018; Reinhart, Mardaljevic, \& Rogers, 2006; Wienold \& Christoffersen, 2006). An example of this is the EN17037 standard (2018, p. 5), which "specifies the elements for achieving, by means of natural light, an adequate subjective impression of lightness indoors, and for providing an adequate view out". And yet, right after 
such a statement which emphasizes subjective satisfaction, it reduces its scope to nearly measurable aspects when stating that it "gives information on how to use daylighting to provide lighting within interiors, and how to limit glare".

This reduction of visual comfort to an almost exclusively physiological phenomenon implies that some well-known evidence is impossible to explain. For instance, evidence suggests that attractive views increase people's tolerance for visual discomfort (Aries, Veitch, \& Newsham, 2010). However, since the luminance distribution (i.e., the physical quantities in the environment) remain constant, this variation in Visual Comfort can only be explained by referring to "the mind", which is not accounted for by current conceptual frameworks. Consequently, there is no clear theoretical explanation as to how visual comfort relates to the attractiveness of outside views. In fact, most empirical models of visual comfort disregard views altogether (Jakubiec \& Reinhart, 2012; Konstantzos \& Tzempelikos, 2017; Wienold, 2009; Wienold \& Christoffersen, 2006) and the efforts to assess them simultaneously do not combine them into a single score or index and limit themselves to report the two variables in parallel (EN17037, 2018; Hellinga, 2013; Hellinga \& Hordijk, 2014).

The acoustic domain is different from the thermal and daylight ones because, in it, comfort "is [a] vaguely defined and explored concept in the literature" (Vardaxis, Bard, \& Persson Waye, 2018, p. 152). Instead, a distinction is made between sound as a general term and noise, the latter referring to those unwanted sounds that are "distracting, annoying, or harmful to everyday activities (work, rest, entertainment, or study)" (Doelle, 1972, p. 137). This distinction between unwanted and other kinds of sounds can be used to define what acoustic comfort is. For instance, Vardaxis (2018) argues that one of the relevant definitions of acoustic comfort is the "absence of unwanted sound and opportunities for acoustic activities without annoying other people", given by Rindel (2002, p. 1). From this perspective, and considering that people tend to ignore noises that are produced by their own activities (Doelle, 1972, p. 137), acoustic standards, recommendations and guidelines often suggest limiting the external and/or background noise (Cowan, 1994; Doelle, 1972, p. 185; R. J. Peters, Smith, \& Hollings, 2011).

The very definition of noise as an unwanted sound, suggests that acoustic comfort is a subjective state of the mind. Moreover, the fact that Rindel's (2002, p. 1) definition emphasizes that people do not want to bother others (i.e., accounting for annoyances on other people's bodies) reflects that acoustic comfort is not constrained to simple physiological phenomena. Similarly, Egan (1988, p. 21) argues that hearing perception varies from person to person. He explains this by saying that "sound intensity is not perceived 
directly at the ear; rather it is transferred by the complex hearing mechanism to the brain where acoustical sensations can be interpreted as loudness" (1988, p. 21). Similarly, (Doelle, 1972 , p. 19) states that if a "noise is uninterrupted and is not too loud, and if it has no information content, it will become acceptable background noise and will suppress other objectionable intruding noises, making them sound psychologically quieter". The requirement for this noise to have no information is probably related to the fact that, as stated by Angevine (1972, p. 179), the meaning given to a noise directly impacts how pleasant it is perceived to be.

Despite the accepted subjective nature of acoustic comfort, the literature often quickly reduces this concept to a pseudo-objective construct. For instance, Egan (1988, p. 29) ends up operationalizing the perception of loudness by assuming that it depends only on two physical factors: sound level (i.e., decibels) and frequency. The same happens to Rindel (2002), who ends up providing design criteria for acoustical comfort that depends only on physical parameters. Likewise, Vardaxis and colleagues (2018a; 2018) end up ignoring psychological elements of comfort and focusing only on more objective elements such as frequencies, sound intensity levels, and sources. Vardaxis and Bard also argue that "subjective response to airborne sound in dwellings is complicated: it can be predicted well in some cases but not always" (2018b, p. 303). They specifically mention that music and speech are perceived differently, but instead of attributing these differences to what the noise represents to people (as stated by Angevine, 1972), they quickly relate them to the much more objective frequency spectrum of the different sounds. Similarly, several books approach acoustic phenomena from a mathematical perspective (e.g., Cowan, 1994; Peters et al., 2011) and others purposefully avoid doing so (e.g., Doelle, 1972, p. ix).

Finally, the literature on air Quality tends to focus not only on people's comfort and satisfaction (ASHRAE, 1999; Fanger, 1989; Mickaël et al., 2014) but also on their productivity and performance (Mujan et al., 2019) and health (ASHRAE, 1999; Mujan et al., 2019; Sundell, 2004). As in the other domains, the literature refers to comfort in terms that emphasize subjectivity but then claim or assume that the concentration of pollutants is the exclusive cause of people's subjective responses. For instance, Fanger (1989) states that people are often bothered by and thus complain about the air quality in buildings, suggesting that the perceived air quality of air is subjective. However, he later introduces a "comfort equation" that relates the perceived air quality with the sources of pollutants inside of a space. Similarly, the ASHRAE standard 62 (1999, p. 2) defines an acceptable air quality as that in which "there are no known contaminants at harmful concentrations as determined by cognizant authorities and with which a substantial majority $(80 \%$ or more) of the people 
exposed do not express dissatisfaction". This direct relationship between pollutants and comfort is a conscious simplification as the same standard (1999, p. 2) states that "many other factors... may affect occupant perception and acceptance of indoor air quality, such as air temperature, humidity, noise, lighting, and psychological stress".

In summary, while in principle building sciences sees comfort as a subjective state of the mind, in practice this concept ends up being a much simpler and more objective construct. Specifically, building science commonly quantifies comfort through equations that depend only on objectively measurable variables of the environment and physical personal elements. The following section argues that this has been possible by consciously disregarding some phenomena documented in the literature. This argument will be presented while explaining the process through which comfort develops.

\subsection{Understanding how comfort develops with and without "the mind"}

The previous section demonstrated that in the bulk of building science literature, comfort is argued to be a state of the mind but that, in practice, its working definition tries to ignore "the mind" as much as possible. That section also stated that this is carried out by developing models that depend only on variables that can be relatively easily measured and/or quantified. This paradigm is implicit in a great portion of the literature and often shapes it. For instance, Bluyssen's (2009) book about indoor environment has a section on Human Perception and Reception in which the discussion about human cognition is dwarfed by that of physiological processes. Similarly, Bean's (2020, p. 114) book on thermal comfort has a chapter dedicated to thermal Comfort and Human Physiology and Psychology which greatly emphasizes the human body's thermoregulatory system but almost completely ignores "the mind".

But, if comfort is a state of the mind, how does the environment manage to shape such a state? Moreover, if comfort is a state of the mind, how can research produce useful knowledge about comfort while ignoring the mind? This section explains the process through which people's comfort develops, according to the literature. This review reveals how building science makes sense of comfort while virtually ignoring the mind. It also identifies the elements that are known to influence people's comfort, information that was very helpful for designing this research.

\subsubsection{Sensations: from the environment into the human body}

The literature appears to agree that the first stage in the development of comfort involves a set of sensory receptors that can transform environmental variables into signals that the 
human brain can interpret. This first contact between environment and body is known as sensation (Coren, Ward, \& Enns, 2004), a terminology that this dissertation will continue using.

As an example, Bluyssen (2009, p. 4) argues that "the human senses... are basically the instruments we have to report or indicate whether we feel comfortable... in terms of acceptability with respect to heat, cold, smell, noise, darkness, flickering light, etc.". Furthermore, Bluyssen states that "through sensory receptors, via nerves (the spinal cord and cranial), the senses can provide information to the brain, which is processed and used to send messages with prescribed actions to the relevant parts of the human body" (2009, p. 9). Within the lighting domain, Reinhart (2014) emphasizes this connection between the environment and the human body by dedicating one chapter to the source (i.e., the sun and sky) and another to the sensor (i.e., the human eye). In the acoustic domain, Egan (1988, p. 21) states that "sound intensity is not perceived directly at the ear; rather it is transferred by the complex hearing mechanism to the brain where acoustical sensations can be interpreted"; and Cowan (1994, p. 25), that "each stimulated cell sends an electrical signal to the auditory nerve. The actual frequency interpretation takes place after the stimulated hair cells send their respective electrical signals through the auditory nerve to the brain."

Outside of building science, Schiffman (2000, p. 1) states that "virtually everything we know about the environment is based on a pattern of physical energies that directly affect our sensory receptors". Likewise, Feher (2012) explains that people's sensory systems connect the central nervous system with the events outside of it. This includes not only the external world but also the receptors that inform the central nervous systems about what is happening inside the human body. Also, Li and Wong (2006, p. 9) argue that physical processes provide signals that people's sensory receptors translate into neurophysiological impulses. It is worth mentioning that this stage is purely physiological. That is to say, even if sensations might fluctuate from person to person, these differences are the result of physical/physiological variability across individuals and of their non-physical personal factors.

\subsubsection{Perceptions: getting into the brain}

After people's sensory receptors produce a sensation, their brain will often generate a perception; that is, an awareness of the external stimulus (Feher, 2012) that people will interpret (de Dear, 2011). This implies that, contrary to sensations, perceptions require cognition (Coren et al., 2004) and thus they possess a strong psychological component. This is clear, for instance, in the framework about adaptive thermal comfort developed by Luo 
(2020) and the model by Yao et al. (2009), that distinguish between the physiological and psychological response to the stimulus from the thermal environment. The fact that sensations precede perceptions is also patent in the psycho-physiological model of thermal comfort presented by Auliciems $(1981,1983)$, where the physiological thermoregulation is considered an intermediate step between satisfaction and the thermal environment. What these models do not consider, however, is that not all sensations have an intensity capable of eliciting perceptions (Feher, 2012). This is a well-known fact that underlies the concept of absolute threshold, which refers to the minimum stimulus necessary for an organism to detect it. Stimuli weaker than this threshold is called subliminal and will not be noticed (Schiffman, 2000, p. 24). The acoustic domain does acknowledge the existence of an absolute threshold, which is evidenced by the fact the decibel scale it uses is anchored (e.g., the value of zero is defined to be at) at the minimum perceivable sound pressure (Cowan, 1994, p. 31).

Perhaps because they fail to consider the existence of the absolute threshold—which would mean that all sensations necessarily elicit perceptions-building scientists tend to use these concepts interchangeably (a common misunderstanding, according to Coren et al., 2004, p. 8). For instance, since it is the result of a cognitive process that interprets sensory data to describe a space as being "too hot" or "too cold", thermal sensation should be called thermal perception. Despite this, the lack of precise language does not necessarily imply that the building science literature completely ignores the differences between these two concepts. For instance, studying thermal comfort in situations where different parts of people's body are exposed to different temperatures implies understanding that it is the brain who will, at some point, aggregate all these "local thermal sensations" into a single "whole-body thermal sensation" (Arens, Zhang, \& Huizenga, 2006a, 2006b; Zhang, 2003; Zhang, Arens, Huizenga, \& Han, 2010b, 2010a). Thus, this deficiency probably relates more to a lack of clear and precise terminology than to a lack of knowledge.

Despite knowing the difference between perceptions and sensations, building scientists often treat them-amalgamated into a single concept—as if they were nearly objective (e.g., Djongyang et al., 2010; Fanger, 1989; Schweiker et al., 2017). For instance, several metrics have been developed for quantifying glare Jakubiec \& Reinhart, 2012; Konstantzos \& Tzempelikos, 2017; Sarey Khanie et al., 2017; Sawicki \& Wolska, 2015; Wienold, 2009; Wienold \& Christoffersen, 2006), a perception which is said to be "caused by excessive light or contrast in a field of view" (Jakubiec \& Reinhart, 2012, p. 150), disregarding other potential sources of variability. This means that, even if building science knows that perceptions do not only depend on sensations but also other more cognitively-driven factors, its models of comfort have mostly ignored all elements that cannot relatively easily be treated as objective. 
Similarly, people's thermal sensation is known to be affected by non-thermally-relevant factors like the amount of daylight in a space (Chinazzo, Wienold, \& Andersen, 2019), the colour of the light (Chinazzo, Wienold, et al., 2018a; Golasi, Salata, Vollaro, \& Peña-García, 2019), and by the physical appearance and the knowledge of the internal temperature of a room (Rohles, 2007). Likewise, people seem to interpret noise differently depending on what it represents (Angevine, 1972) (e.g., music vs speech, according to Vardaxis and Bard, 2018b). For instance, "a child practicing the piano may be a musical treat to his father while the neighbours contemplate calling the police” (Doelle, 1972, p. 137). Similarly, air temperature and people's psychological stress seem to influence the perception of air quality (ASHRAE, 1999), and the control that people have over the environment can affect both thermal and visual perceptions (Luo et al., 2014; Veitch, 2001). However, even if building scientists seem to know all this, these components are not explicitly part of current comfort models or frameworks, which might partially explain why these model's predictions are not as precise as they could be (Cheung, Schiavon, Parkinson, Li, \& Brager, 2019; Pierson et al., 2018). The loss of accuracy induced by ignoring this factor, however, is unknown. For instance, it is unclear whether the influence of the colour of daylight and appearance of a room over people's thermal sensation is noticeable under standard conditions in which the colour of the light and the appearance of spaces do not change that much (G. Chinazzo, personal communication, February 27, 2019).

It is worth mentioning that some frameworks do acknowledge that "the mind" affects perceptions. However, they do this without any explicit theoretical foundation in their models. For example, trying to predict people's overall comfort or satisfaction based on environmental stimuli (as done by Buratti, Belloni, Merli, \& Ricciardi, 2018; Franzitta et al., 2014; Ricciardi \& Buratti, 2018) do not make sense unless there is a psychological component. Despite this, these efforts seem to rely more on correlations and statistical tests than on the validation of models that reflect causal relationships between variables and thus "the majority of [these] studies appear to practically start from scratch" (Schweiker, Ampatzi, et al., 2020, p. 15). Studies like the ones by Kim and de Dear (2012) and Martellotta et al. (2016) could potentially be considered exceptions to this because they use Kano's model of satisfaction (Sauerwein, Bailom, Matzler, \& Hinterhuber, 1996). However, Kano's model is not a comfort model and thus no causal relationship between different components of comfort is ever justified. On the contrary, these studies use Kano's model almost exclusively to argue that the effect of the different comfort factors - e.g., glare, thermal sensation, etc.over people's overall satisfaction does not have to be linear. For instance, Kim and de Dear (2012) found that the negative impact of an unpleasant temperature is greater than the 
positive impact of a pleasant temperature. This is similar to what Arens et al. (2006b) suggest when they stated that people tend to evaluate "thermally neutral conditions" only as "comfortable" and not as "very comfortable". Despite the relevance of these findings, there seems to be no underlying comfort model or theory behind these studies.

Likewise, adaptive thermal comfort models include a component of adaptation and expectation because they are calibrated through extensive field survey data (Brager \& de Dear, 1998; Humphreys et al., 2016; Nicol et al., 2012), and thus they capture the tendency of people to adapt themselves and to adjust the environment in which they are immersed in. For instance, people modify their clothing (Humphreys, 1979) and expectations (Luo et al., 2019), and also operate the windows and heating devices when they are feeling uncomfortable. Despite this, adaptive comfort models pretend to incorporate all these components_-behavioural, psychological and physiological—through a simple relationship between physical quantities. The result is a statistical model-i.e., an equation representing how comfort temperatures vary with exterior temperature (Humphreys et al., 2016; Nicol et al., 2012) — that neither explicitly incorporates individual variables as inputs nor explains how "the mind" operates.

\subsubsection{Comfort: from the brain to the mind}

The final stage in the development of comfort is the stage in which the brain "indicate[s] whether we feel comfortable... in terms of acceptability with respect to heat, cold, smell, noise, darkness, flickering light, etc.” (Bluyssen, 2009, p. 9). In simple words, the development of comfort itself.

As suggested earlier, building science literature very often interprets people's comfort as the result of perceptions alone or simply the direct consequence of environmental stimuli (implicitly supporting the existence of sensations). For instance, visual comfort has been defined in terms of environmental conditions and perceptions as "the absence of discomfort, such as glare, insufficient visual contrast or the presence of visible direct sunlight" (Jakubiec \& Reinhart, 2015, p. 2).

That definition reveals that visual comfort is not related to the quantity but the quality of light in a space. This means that there needs to be sufficient daylight to provide enough visual contrast, while also preventing glare. To ensure the former, researchers have proposed different metrics, all depending exclusively on objectively quantifiable physical factors (Andersen et al., 2013; Dogan \& Park, 2017; IESNA, 2012; Nabil \& Mardaljevic, 2005, 2006; Reinhart et al., 2006; Rockcastle \& Andersen, 2012). Likewise, plenty of models intend to 
estimate glare (Jakubiec \& Reinhart, 2012; Konstantzos \& Tzempelikos, 2017; Sarey Khanie et al., 2017; Wienold \& Christoffersen, 2006), all of which depend solely on objectively quantifiable physical factors (Pierson et al., 2018). Furthermore, since predicting glare is often computationally expensive, it is a common practice to use illuminance-based metrics as proxies for comfort indicators (Wasilewski, Grobe, Wienold, \& Andersen, 2019) which, again, rely solely on physical quantities of the environment.

These two sets of indices have often been combined into single guidelines. For instance, both the IES LM-83 (2012) and the EN17037 (2018) standards intend to balance the availability of enough daylight while minimizing glare. One difference between the two is that, while the latter considers that the presence of some direct sunlight is a good thing, the former suggests avoiding it as much as possible. This last strategy is not rare. In fact, "the most common design recommendation is to 'avoid direct sunlight" (Jakubiec \& Reinhart, 2015, p. 2).

As acknowledged by the EN17037 (2018) standard, avoiding direct sunlight altogether is a questionable decision. For instance, it is known that the availability of contrast and direct sunlight can make a space look more interesting and appealing (Chamilothori et al., 2019; Dogan \& Park, 2019; Rockcastle et al., 2017; Rockcastle \& Andersen, 2012). Based on this idea, the framework described by Dogan and Park (2017, 2019) and Park and Dogan (2019) adapts the LM-83 (2012) standard to acknowledge that, within residential contexts, direct sunlight contributes to the attractiveness of spaces. However, this approach does not fit easily within the definition of visual comfort. It ensures that some direct sunlight is available in the space, without any coherent theoretical explanation of why that particular amount or how it affects people's subjective comfort. Something similar happens with the EN17037 (2018) standard, whose goal is to help providing a subjectively good daylight (assessed through quantifiable factors) and views and, "in addition, recommendations for the duration of sunshine exposure" (2018, p. 5).

In contrast to visual comfort definitions, which mainly rely on physical factors and perceptions, thermal comfort definitions often quite strongly embrace "the mind" (e.g., ASHRAE, 2010, p. 3; de Dear, 2011, p. 111; Hensen, 1991, p. 2.4; ISO, 1984, p. 4). Despite this, it seems to be a common practice to disregard all subjectivity by equating thermal comfort to a neutral thermal sensation. For instance, models built from the "rational" approach to thermal comfort (Djongyang et al., 2010) mathematically estimate the average thermal sensation of large groups of people and, from it, the percentage of the population that might feel uncomfortable (Carlucci, Bai, de Dear, \& Yang, 2018). These models literally 
posit that people's thermal sensation is only the result of thermodynamic balances or imbalances that prompt neutral and non-neutral thermal sensations, respectively. In other words, these models not only assume that a neutral thermal sensation implies thermal comfort, but that this sensation is the result of only thermodynamic phenomena. Probably the most notable exception to this was the extension of the original model to account for people's expectations (Fanger \& Toftum, 2002). Still, rational models still assume that people whose thermal sensation is within the "slightly warm" to "slightly cold" range are comfortable.

The adaptive approach to thermal comfort (Brager \& de Dear, 1998; Humphreys et al., 2016; Nicol et al., 2012) — which is the alternative to the rational (i.e., thermodynamically driven) approach to it-incorporates much more of the psychological elements of comfort. While this is a great step towards the incorporation of "the mind" (de Dear, 2004), adaptive comfort models still often focus on finding "thermal neutralities (comfort temperatures)... by regressing thermal sensation votes on concurrent indoor air temperatures" (Parkinson et al., 2020, p. 6). It would seem, then, that while adaptive comfort models have incorporated psychological phenomena, they still rely almost exclusively on thermal sensation (i.e., a perception) for predicting thermal comfort. In fact, the official website for the ASHRAE Global Thermal Comfort Database II (Földváry Ličina et al., 2018) which contains field data gathered by a variety of scientists from all around the world (de Dear, 1998; Földváry Ličina et al., 2018) warns that less than $30 \%$ of the records contain information about people's subjective comfort or thermal acceptability (ASHRAE, 2021).

In the acoustic and air quality domains, on their part, comfort tends to be vaguely defined and thus the absence of unwanted sound (Vardaxis et al., 2018) and indoor pollutants (ASHRAE, 1999; Fanger, 1989) are used as indicators, respectively. For instance, acoustic comfort is associated with "conditions with little noise and disturbances in a certain space" (Vardaxis et al., 2018, p. 152). Likewise, noise exposure seems to be used as a proxy for acoustic discomfort even if factors such as demographic and societal factors are known to matter (Andargie, Touchie, \& O’Brien, 2020; Laszlo, McRobie, Stansfeld, \& Hansell, 2012). An acceptable indoor air quality, in its part, is defined in ASHRAE standard 62 (1999, p. 2) as one with "no known contaminants at harmful concentrations... and with which a substantial majority ( $80 \%$ or more) of the people exposed do not express dissatisfaction". This conceptualization of comfortable air quality as an absence of pollutants is mainstream (Fanger, 1989; Mickaël et al., 2014).

So, in summary, it would seem that building science's conceptualization of comfort has 
disregarded most of the non-physical personal variables and cognitive processes in order to favour more objective elements. Specifically, comfort models often depend exclusively—or at least too strongly - on perceptions that can be estimated from physical quantities of the environment and treated as if they were objective. The following section posits that a likely root for this simplification is the fear of the potential chaos that embracing cognition can bring to building science's research and practice.

\subsection{Why "the mind" is mostly ignored}

Section 2.1 argued that, while in principle "comfort is a state of the mind", building science commonly simplifies this definition. As argued in Section 2.2.3, this discipline tends to adopt a definition of comfort that too strongly depends on relatively more objective perceptions or just on environmental conditions. This effectively creates two conflicting paradigms: either comfort is a state of the mind, or it just depends on objectively measurable elements of the environment.

It would seem that these two paradigms have managed to coexist within the same discipline only because one is considered the simplified version of the other. One corresponds to "the real world" and the other is the result of a process of simplification of "the real world", natural to the development of any model. The purpose of this simplified model is to help perform research and practice better. As stated by Hellinga (2013, p. 2), "researchers try to limit the number of variables in each experiment, because it is more difficult to detect cause and effect relationships between many different variables".

In the case of daylight research, for instance, there seems to be "fear that a quality assessment of e.g., the 'visual interest of a space' leaves too much room for subjective interpretation" (Reinhart, 2014, p. 27). Likewise, thermal comfort research focuses much more on thermal neutrality (e.g., feeling neither cold nor hot) than on subjective comfort. The reason for this might be, in the words of Djongyang, Tchinda and Njomo (2010, p. 2627), that "satisfaction with the thermal environment is a complex subjective response to several interacting and less tangible variables. In other words, there is really no absolute standard for thermal comfort. [However] in general comfort occurs when... physiological regulation is minimized". Similarly, Auliciems (1981, p. 113) argues that "under thermally near neutral laboratory conditions, minimally experienced temperature sensation, discomfort, and, an even more integrated response of 'unpleasantness'... are coincident". Finally, Egan (1988, p. 21) explains that sensitivity to noise depends on several factors and yet he presents a table that serves as a guide to explain how sound increases and decreases in different architectural situations. 
In the end, all models are simplified representations of "the real world", meaning that they disregard some relevant inputs and thus their predictive power is always limited. For instance, O’Brien, Gunay, Tahmasebi, \& Mahdavi (2017) argue that, when modelling occupants, building science models often suppress diversity by focusing solely on representative individuals. This is also evident in the ASHRAE standards 55 and 62 which claim that some people might not find the environmental conditions comfortable due to occupant diversity (ASHRAE, 1999, 2010). This reveals that quantitative comfort models tend to estimate how comfortable an average individual would be in certain scenarios, knowing that some people will not be represented by its results. For example, Lawrence Race states that "decisions on design conditions are made harder by the fact that comfort is a very subjective response with different people having different comfort levels; so the main aim is literally to 'keep most of the people happy most of the time"' (2006, p. 1). In other words, "due to individual differences it is impossible to specify a thermal environment that will satisfy everybody... There will always be a percentage of dissatisfied occupants" (ISO, 1984, p. 4). However, "it is possible to specify environments known to be acceptable by a certain percentage of the occupants." (ISO, 1984, p. 4). Similarly, Egan (1988, p. 29) provides curves of loudness level for different frequencies, warning that "because variation occurs from individual to individual, the curves represent averages for many subjects".

It is worth noticing that the predominant building science approach to developing comfort models is similar to the one that other disciplines take for different kinds of problems. For instance, Train (2002, p. 3) states that, since some factors that affect people's choices are unobserved, these are "not deterministic and cannot be predicted exactly. Instead, the probability of any particular outcome is derived". However, this still presents a contradiction because, after the great emphasis given to "subjectivity" and "the mind" (see Section 2.1), comfort models in building science seem to suggest that both the subject and the mind can be ignored without great loss of predictive capacity.

To summarize, it seems to be that the reason why building science has disregarded "the mind" is not its lack of rigour, but pragmatism. Models are simplified representations of the real world, and thus modelling will always carry a trade-off between the complexity and usability of the model. Specifically, it would seem that models often do not consider "the mind" because they would just become too complex and because it would depend on several factors that are impossible to observe. Nevertheless, it is not necessarily true that embracing "the mind" will introduce chaos. The next section argues that models that consider cognitive processes are likely to be more complex, but they will not necessarily be unusable. 


\subsection{Embracing "the mind" through exploratory research}

building science has historically modelled comfort based on objectively quantifiable physical factors (e.g., ASHRAE, 2010; Bean, 2020; Wienold et al., 2019). This association effectively reduces subjectivity and disregards most of "the mind"-i.e., the non-physical personal factors and relevant cognitive processes-from the concept of comfort. This is a simplification that, as concluded in Section 2.2, building science carries out by explaining comfort almost exclusively in terms of human perceptions driven by environmental stimuli with people's comfort (e.g., glare, thermal sensation, loudness, etc.). However, Section 2.3 concluded that this simplification is made not due to a lack of rigour but due to pragmatism, meaning that the complex concept of comfort is simplified to make comfort models usable. This section discusses how this simplification is reflected in building science's comfort research. It argues that it is possible to embrace more of "the mind" through more exploratory and qualitative methods and that doing so might not result in overly complicated models and overwhelmingly chaotic and incoherent data.

\subsubsection{Current practices: confirmatory vs exploratory research}

building science's comfort research tends to be confirmatory because it tends to focus on building correlations between people's responses to questionnaires and a set of measured environmental conditions (e.g., de Dear et al., 2016; Wienold \& Christoffersen, 2006), or by — in the context of an experiment—asking them to adapt predefined aspects of a room to make it more comfortable (e.g., Van Den Wymelenberg \& Inanici, 2009). This approach is not only common to single-domain studies (i.e., focusing on either thermal, visual, acoustic or air quality) but also on multi-domain studies (Schweiker, Ampatzi, et al., 2020). This practice is confirmatory because scientists need to choose, a priori, the factors that explain comfort and then confirm - through mathematical methods and statistical tests-whether these factors affect comfort, and estimate the magnitude of their influence. Grounded in this kind of procedure, it is uncommon to see building science research that relies on asking open-ended questions to non-experts. For example, Mahdavi et al. (2021) found that only $11 \%$ of the articles documenting the role of people on the difference between the estimated and actual energy consumption of buildings utilized interviews. Most of them relied on surveys, observations or sensor-based measurements.

For instance-instead of letting non-experts speak freely-thermal comfort research generally involves asking participants to answer "how they feel right here right now" through a simple bipolar adjectives item that goes from 'hot' to 'cold' passing through 'neutral' (e.g., de Dear, Kim, \& Parkinson, 2018; Song et al., 2018). The reason for asking this question is 
just that there is no other way of acquiring (e.g., measuring) such information (Luo, 2020, p. 7). This self-reported subjective perception is known as thermal sensation and has the advantage of being "a rational experience that can be described as being directed towards an objective world" (Hensen, 1991, p. 2.4). In other words, even if it is self-reported-i.e., subjective-building scientists tend to treat people's self-reported thermal sensation as an objective value because the question used to assess it leaves very little room for people's subjective interpretations (e.g., Djongyang et al., 2010; Fanger, 1989; Schweiker et al., 2017). This is the case for both the rational (Auliciems \& Szokolay, 1997; Cheung et al., 2019) and adaptive (Brager \& de Dear, 1998; Humphreys et al., 2016; Nicol et al., 2012) approaches to thermal comfort and also for comfort research in other domains. For instance, research about glare (e.g., Konstantzos \& Tzempelikos, 2017; Sarey Khanie et al., 2017; Wienold et al., 2019) and even studies that involve a high subjective component (e.g., Cauwerts \& Bodart, 2011; Chamilothori et al., 2019; Newsham, Richardson, Blanchet, \& Veitch, 2005; Rockcastle et al., 2017) tend to use Likert-scale questions for gathering data with which to test mathematical models and equations.

Unfortunately, confirmatory research methods are not the most effective for understanding the role of "the mind" in the development of people's comfort. What should researchers ask in their questionnaires? What variables should they measure or vary in their experiments? The answer to these questions can be obtained through exploratory research. That is to say, through methods that allow gathering data that researchers were not expecting. For example, by asking people open-ended questions.

While uncommon, some building science studies have successfully asked open-ended questions to non-experts as part of their methodology. For instance, Attia (2020) used indepth interviews to understand how people's behaviour could explain the mismatch between estimated and actual energy savings after residential energy efficiency improvements. Similarly, Bend and Sunikka-Blank (2015) used semi-structured interviews to understand the variety and complexity of people's heating behaviour. Outside of building science, on the contrary, asking open-ended questions to non-experts is a common practice (Fontana \& Frey, 2005; Johnstone, 2017; Trocchia, Swanson, \& Orlitzky, 2007). For instance, interviews and focus groups can be useful for exploring phenomena before developing surveys that allow performing quantitative analyses (Creswell, 2014). One example of this is the use of interviews for eliciting a population's commonly held Attitudes, Subjective Norms and Perceived Control associated with a certain behaviour (Francis et al., 2004). These findings can later be translated into a questionnaire based on the Theory of Planned Behaviour (Ajzen, 1985, 1988, 1991). Similarly, semi-structured interviews have been useful for understanding 
what people know and how they reason, including the environmental cues and strategies they use for making decisions (Crandall \& Hoffman, 2013). Also, and perhaps more closely related to the topic of this research, in-depth interviews have been used for getting insight into people's perceptions of quality and value (Zeithaml, 1988); and focus groups, for understanding why people's attitudes and self-reported behaviour do not always match their actual behaviour (Johnstone \& Tan, 2015).

Despite the existence of some exceptions (e.g., Attia, 2020; Ben \& Sunikka-Blank, 2015) and of it being routine in other disciplines, allowing non-experts to express themselves and articulate what they want or need is an uncommon practice in building science. As sometimes evidenced (e.g., Tamas, O’Brien, \& Gunay, 2018), "self-reported behavior may not always match observed behavior" (Yan \& Hong, 2018, p. 13). Even worse, "regardless of the accuracy of self-reporting in surveys, participants may not have the insight or knowledge required to identify the subtle building characteristics that are known by researchers to influence comfort" (O’Brien, Schweiker, \& Day, 2019, p. Introduction). So, in summary, building science sees people's responses as highly naïve, unreliable and/or untruthful.

This attitude towards non-experts' responses is partly justified. After all, by definition, non-experts do not have any theoretical knowledge about building physics or comfort (Crandall \& Hoffman, 2013) and thus their responses might be based on their oftenunreliable intuition (Kahneman, 2003). However, it is important to distinguish between the theoretical knowledge that explains why or how something happens from the intuitive knowledge that can be used to predict whether something will happen. For instance, while it is sensible not to expect lay-people to provide knowledge on the former, they might be very capable of offering insights on the latter because intuitive judgments can, under certain conditions, be trusted (Bago \& De Neys, 2018; Crandall \& Hoffman, 2013; Kahneman \& Klein, 2009). The remainder of this section will formalize the definition of intuition that this dissertation uses (Section 2.4.2) and also explains why people's intuitive judgments of what makes them comfortable are likely to be trustworthy (Section 2.4.3). (Which, as suggested earlier, does not mean that their responses related to why or how they become comfortable can be trusted.)

\subsubsection{A formal definition of intuition}

By reviewing several previous studies, Stanovich and West (2000) found that several theorists in the discipline of psychology had proposed the coexistence of two human reasoning systems. The first system-System 1-is mostly automatic, largely unconscious, and requires low cognitive effort; while the second system-System 2-relates to the analytic 
intelligence that has most traditionally been studied (Kahneman, 2003; Stanovich \& West, 2000). Systems 1 and 2 are also called intuition and reasoning (Kahneman, 2003), automatic and reflective systems (Thaler \& Sunstein, 2008), and some other names by others (Evans, 2003, 2008). Although "System 1" and "System 2" are the most formal names (Evans, 2003), this dissertation will use the more explicit names of intuition and reasoning. This makes it easier for the reader to remember the characteristics of each system. An intuitive impression of the characteristics of both systems might be inferred from the fact that intuition is often said to be present in both humans and animals, while reasoning is supposed to be unique to humans (Evans, 2003).

While fast and easy to build, intuitive judgments cannot always be trusted. These judgments are often emotionally charged and governed by habits, thus difficult to control and modify (Kahneman, 2003). Even worse, people do not know which cues they are using to build these judgments, and subjective confidence is not a reliable indicator of their validity (Kahneman \& Klein, 2009). For instance, there is a classic mathematical problem that states: "a bat and a ball together cost $\$ 1.10$. The bat costs $\$ 1$ more than the ball. How much does the ball cost?" Most people's intuitive system jumps to answer this question by saying that the ball costs 10 cents (Bago \& De Neys, 2018; Kahneman, 2003; Thaler \& Sunstein, 2008), which is not the correct answer. This not only suggests the existence of a highly impulsive intuition but also shows that a great number of people will not check their intuitive judgments for validity (Kahneman, 2003).

While not explicit, the role of intuition is present in comfort research. For instance, Bluyssen (2009, p. 9) argues that "the human senses are essentially the instruments we have to report or indicate whether we feel healthy and/or comfortable, knowingly (consciously) or unknowingly (unconsciously)". Likewise, Rohles (2007) reports the result of an experiment in which people reported different thermal sensations when standing in two rooms at the same temperature. He explains the results by stating that "Chamber B was an $8 \mathrm{ft}$ by $10 \mathrm{ft}(2.4 \mathrm{~m}$ by $3 \mathrm{~m})$ walk-in commercial refrigerator and anyone knows (recall the role of past experience and state of mind) that when you go into a refrigerator you feel cold"(2007, p. 22). Rohles (2007) also explains how people changed their thermal sensation depending on what temperature they were told the room was at. This can be interpreted as people's brains sending signals, suggesting that "it should be cold".

But, if people's intuitive responses are often wrong, why would building scientists trust the information given by them? One reason is that intuition is not necessarily always wrong (Bago \& De Neys, 2018). As discussed in the next section, people's intuition can be quite a 
reliable source of knowledge for the building science discipline. This implies that people's will often agree on an answer-arguably the correct one-and thus asking people questions will not necessarily lead to an infinite number of interpretations of comfort. In other words, embracing "the mind" will not necessarily result in overly complex models of comfort that are impossible to use in practice.

\subsubsection{Why embracing "the mind" will not necessarily result in chaos}

As mentioned earlier, people often make intuitive judgments that are difficult to control and modify (Kahneman, 2003). However, and more importantly, these judgments are not always wrong (Bago \& De Neys, 2018). Intuition can be trusted when trained through prolonged practice and feedback in what Kahneman and Klein (2009) call "high-validity environments". That is to say, environments in which there is a stable relationship between objectively identifiable cues and the subsequent events. These systems with stable causeeffect relationships (like most physical systems that people interact with) allow individuals to learn their governing rules. On the contrary, intuition should not be trusted in low-validity environments where the causes and effects are much more difficult to predict. In those systems, the hypothetical thinking capabilities of the reasoning system are required. For example, it is impossible to "learn by experience to avoid disasters such as nuclear war or the effects of uncontrolled global warming" (Evans, 2003, p. 454).

Based on Kahneman and Klein's (2009) description, the built environment is a high validity one. After all, the way it behaves and its causal relationships respond to the highly stable laws of physics. Moreover, since people spend a long time within buildings, people are likely to have a highly trained intuition about how the built environment works. This includes knowledge about how the built environment interacts with people's bodies, and therefore they may be able to articulate what is it that they find acceptable, prefer, dislike, or find intolerable. Therefore, people's intuition can be trusted, at least to a certain point. For instance, Schweiker, Rissetto and Wagner (2020) found that people's thermal expectations are fulfilled the majority of the time, suggesting that people can read the cues on the environment to make relatively accurate inferences about it.

It is important to notice, however, that people's explanations of why or how the built environment behaves the way it does will not necessarily be correct. The reason for this is that, while people's highly trained intuition might enable them to appropriately identify which features cause which effects (Kahneman \& Klein, 2009), they will not likely have the socalled "book knowledge" (Crandall \& Hoffman, 2013). In other words, they might lack the knowledge about the theory and models behind physical phenomena in buildings and thus 
they are not expected to know how or why something happens, even if they are sure that they know it (Crandall \& Hoffman, 2013).

So, if people's intuition about what might happen in the built environment are likely to be correct, then they will mostly point in the same direction (i.e., towards what really happens). This implies that asking people to elaborate on what makes them comfortable and when will not result in an infinite amount of different responses. On the contrary, it should lead to a relatively small number of factors that affect people's comfort.

\subsection{Conclusions and chapter summary}

Through a literature review, this chapter has argued that there are two coexisting paradigms of comfort within building science research. In the first one, comfort is a subjective state of the mind that depends only on physical quantities and environmental factors. In the second one, however, comfort is a much simpler concept-almost objective-that depends mostly on environmental parameters and other physically relevant factors. As shown in Section 2.1, these two paradigms not only coexist within the same discipline but often within the same document or even sentence. It seems to be that the second paradigm — the simplest one-dominates both research and practice. In other words, while comfort is, in principle, "a state of the mind", it has been the mainstream practice within the discipline of building science to ignore "the mind"; namely, the non-physical personal factors and the cognitive processes that affect people's comfort.

By studying the process through which comfort develops, Section 2.2 concluded that building science has managed to disregard "the mind" by strongly relying on perceptionsamalgamated with sensations into a single concept-to explain comfort. In other words, even if perceptions depend on cognitive processes, building science treats them as if they depended only on physical factors (e.g., models of glare only depend on light distribution in the field of view) and in the process hide_-or even ignore-perceptions rooted in cognitive processes (e.g., expectations).

Section 2.3 argued that the reason these two conflicting paradigms have managed to coexist is because one of them is seen as an acceptable simplification of the other. In other words, every model is a simplification of reality that needs to choose between being more accurate and being easier to use. It appears that building science has favoured the usability of models but, as argued earlier, at the cost of sending a conflicting message: comfort is a subjective state of the mind but "the subject" and "the mind" can be ignored entirely without much loss the model's predictive capacity. 
Finally, Section 2.4 argues that intuitive judgments-i.e., those strong, automatic, and automatic sentences that people often make-are likely to affect how comfortable people feel. Furthermore, that section also argues that people's intuition is highly trained on the way building physics work and thus it can be trusted to some extent in comfort research contexts. This means that performing more exploratory research-i.e., in which researchers can ask people open-ended questions about what makes them comfortable and when (as opposed to the physical explanations of $w h y$ or how this happens) — will not necessarily result in a chaotic variety of answers, but a manageable number of consistent and agreed upon factors that affect comfort. Asking people open-ended questions, then, is likely to be an appropriate tool for embracing more of "the mind" in comfort research. 


\section{THEORETICAL FRAMEWORK}

"Whether real or imaginary, pain is pain and pleasure is pleasure"

BENJAMIN FRANKLIN, THE ART OF PROCURING PLEASANT DREAMS, 1786

Chapter 2 reviewed the literature and identified a contradiction within building science's comfort research: comfort is known to be subjective, building science disregards most of the complexity of the subject by focusing almost exclusively on physical personal factors (e.g., ASHRAE, 2010). Similarly, while comfort is known to be a state of the mind, most building science comfort models ignore cognition as much as possible treating perceptions as if they were objective (e.g., Djongyang et al., 2010; Fanger, 1989; Schweiker et al., 2017). In other words, while comfort is supposed to be a state of the mind, comfort research has tried to ignore "the mind"-i.e., cognitive processes and relevant non-physical personal factors-as much as possible ${ }^{2}$.

This contradiction leads to the coexistence of two conflicting paradigms: comfort as a state of the mind versus comfort as the result of mostly physical/physiological phenomena. This second paradigm dominates research and practice even if it is known to be overly simplistic. While very often implicit, de Dear and Brager (2002, p. 549) explicitly expose this contradiction when stating that 'acceptability' is rarely defined, but that " it is commonly agreed within the thermal comfort research community that 'acceptable' is synonymous with 'satisfaction', and that 'satisfaction' is associated with thermal sensations of 'slightly warm', 'neutral', and 'slightly cool"'.

The following section develops a theoretical framework that can help to better understand this contradiction. Specifically, it identifies which variables influence the development of people's comfort and the relationships between these variables, emphasizing which elements have been ignored from comfort models, and those that have been implicitly and explicitly incorporated. From this framework, three research questions are formulated.

\footnotetext{
2 As explained in section 1.5, this dissertation uses the term "the mind" to refer to the cognitive processes and the non-physical personal factors that affect people's comfort
} 


\subsection{A framework for understanding how comfort develops}

This section explains the process through which comfort develops, according to the literature. In other words, if comfort is "a state of mind", this section explains how is it that the physical environment manages to affect such a state. Figure 2 shows a visual representation of this process.

\subsubsection{The elements that determine people's comfort}

The first contact between the physical world and people's bodies is called sensation (Coren et al., 2004) and it is the result of sensory receptors transforming external stimuli into signals that the human brain can interpret. Bluyssen (2009, p. 9) explains this process by stating that "through sensory receptors, via nerves (the spinal cord and cranial), the senses can provide information to the brain, which is processed and used to send messages with prescribed actions to the relevant parts of the human body". Sensations, then, depend only on the physical characteristics of individuals and other physical parameters. Figure 2 represents this relationship-widely considered in building science's comfort models-as an effect of the physical world on people's sensations, mediated by the latter's physical characteristics.

After sensory receptors emit a sensation, the brain will interpret the signal developing a perception (de Dear, 2011). While often confused and amalgamated, the main difference between sensations and perceptions is that the latter require cognition (Coren et al., 2004) and thus they can possess a strong psychological component. The distinction between sensations and perceptions - as well as the order in which they occur-has been captured by building scientists (Auliciems, 1981, 1983; Luo, 2020; Yao et al., 2009); although it is not a usual practice. For instance, since it is the result of a cognitive process that interprets sensory data to describe a space as "too hot" or "too cold", thermal sensation should be called thermal perception.

Perhaps partly because of this lack of appropriate language, building scientists often treat perceptions and sensations - merged into a single concept—as if they were nearly objective (e.g., Djongyang et al., 2010; Fanger, 1989; Schweiker et al., 2017). Moreover, the perceptions that have been studied are mainly those that can be easily associated with sensory receptors. For instance, glare (Jakubiec \& Reinhart, 2012; Konstantzos \& Tzempelikos, 2017; Sarey Khanie et al., 2017; Sawicki \& Wolska, 2015; Wienold, 2009; Wienold \& Christoffersen, 2006), thermal sensation (ASHRAE, 2010; ISO, 1984), and loudness (Cowan, 1994; Doelle, 1972; Egan, 1988) can be associated to people’s eyes, skin and ears, respectively. 
Treating these sensation-driven perceptions as objective (i.e., as sensations) might be a good approximation at times. Nevertheless, the literature reveals at least two situations in which this assumption will probably induce great error. The first one corresponds to the influence of physical factors of one domain on the perceptions of another domain. For instance, people's thermal sensation is known to be affected by non-thermally-relevant factors like the colour of the light within a space (Chinazzo, Wienold, et al., 2018a; Golasi et al., 2019). These cross-sensorial effects appear to be driven by cognition. In fact, Auliciems (1981, p. 116) argues that integrating psychological components into comfort models "provides a rationale for the otherwise nonsensical association between thermal perception and colour of surroundings".

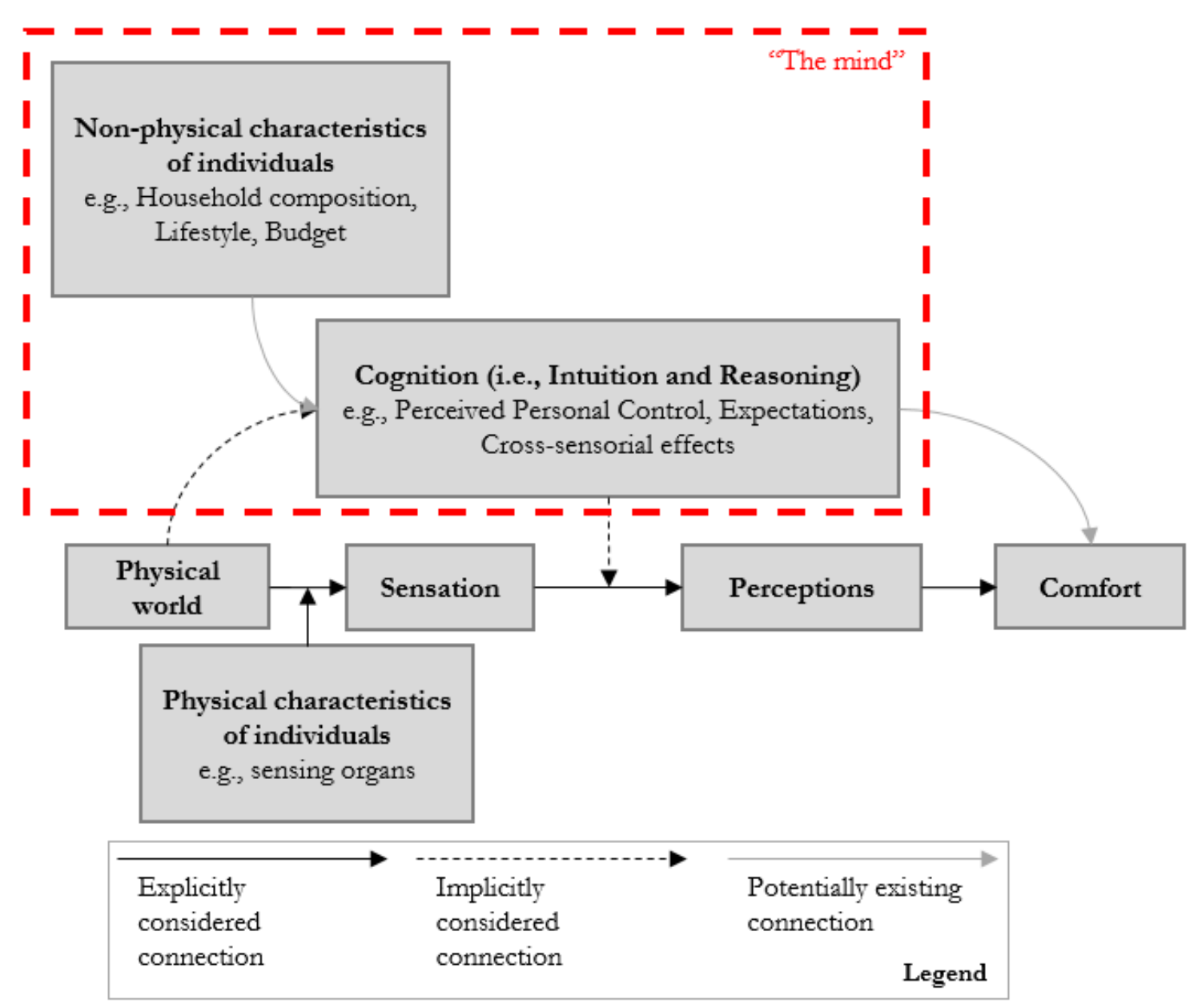

Figure 2: How comfort develops according to the literature

It is worth mentioning that the effect of cross-sensorial elements has been captured in some indices that try to predict people's overall satisfaction with the built environment (e.g., Buratti et al., 2018; Franzitta et al., 2014; Ricciardi \& Buratti, 2018). However, these efforts seem to rely on correlations and statistical tests, and do not always have an underlying theoretical model that reflects causal relationships between variables (Schweiker, Ampatzi, et 
al., 2020). The studies by Kim and de Dear (2012) and Martellotta et al. (2016) are, to some degree, exceptions because they use Kano's model of satisfaction (Sauerwein et al., 1996). However, that model is rooted in the Marketing discipline and thus does not present any causal relationship between different components of comfort.

The second situation in which assuming perceptions to be objective might induce a great error is when they are affected by factors that cannot be easily associated with a sensing organ. For instance, people report different thermal sensations depending on the physical appearance of the space and the knowledge of its internal temperature (Rohles, 2007), and their expectations about it (Luo et al., 2016). Likewise, people seem to interpret noise differently depending on what the noise represents (Angevine, 1972; Doelle, 1972), the control that people have over the environment can affect both thermal and visual perceptions (Luo et al., 2014; Veitch, 2001), and the ASHRAE standard 62 (1999) states that-even if it disregards their effect-psychological stress and other variables can influence the perception of air quality. Moreover, past experiences have been shown to shape people's preferred architectural spaces, stressing the importance of understanding the role that non-physical personal factors play in the development of people's comfort (Roetzel et al., 2020).

Some models have partially acknowledged the effect of cognition over perceptions. For instance, adaptive thermal comfort models (Humphreys et al., 2016; Nicol et al., 2012) acknowledge that perceptions involve some cognition, as they depend not only on behavioural and physiological adaptation but also on psychological ones (Brager \& de Dear, 1998). Despite that, these models do not explicitly include these cognitive processes in their equation. That is to say, these models place all three mechanisms of adaptation behind a correlation that depends solely on physical parameters, effectively putting "the mind" in a black box.

The existence of these two situations - in which cognition seems to significantly affect self-reported perceptions - suggests the relevance of cognition and of "the mind" in the development of people's feeling of comfort. Figure 2 presents cognition as the mediator factor between sensations and perceptions. Examples of the effect of cognition over perceptions are the perceived personal control (Luo et al., 2014; Veitch, 2001), expectations (Luo et al., 2016; Schweiker, Rissetto, et al., 2020) and cross-sensorial factors (Chinazzo, Wienold, et al., 2018a; Golasi et al., 2019). As argued earlier in this section, the effect of sensations over perceptions has been acknowledged by building science's comfort models. Nevertheless, only some models have acknowledged the fact that cognition mediates between the two and have done it only implicitly. 
Finally, building science tends to assume that comfort is the result only of perceptions (Schweiker, André, et al., 2020). For instance, visual comfort has been defined as "the absence of discomfort, such as glare, insufficient visual contrast or the presence of visible direct sunlight” (Jakubiec \& Reinhart, 2015, p. 2). Similarly, “it is commonly agreed within the thermal comfort research community that 'acceptable' is synonymous with 'satisfaction', and that 'satisfaction' is associated with thermal sensations of 'slightly warm', 'neutral', and 'slightly cool”' (de Dear \& Brager, 2002, p. 549). Indeed, at least until recently, "very few studies ha[d] addressed the distinction between sensation and comfort" (Zhang, 2003, p. 3). This also happens within the domain of acoustic comfort (Vardaxis et al., 2018, p. 152).

It is worth asking, however, if it is reasonable to assume that comfort-i.e., a subjective state of the mind-depends only on here-and-now perceptions such as glare, loudness, and thermal sensation. Furthermore, it seems reasonable to question whether people's comfort can be predicted exclusively from here-and-now environmental factors (e.g., de Dear et al., 2016). Figure 2 posits this question by presenting a potentially existing effect of cognition directly into comfort. Similarly, Figure 2 also expresses the fact that some non-physical characteristics of individuals will affect comfort going through cognition. While the fact that some personal psychological elements can affect people's cognitive processes is not very controversial (e.g., the role of past experience identified by Roetzel et al., 2020), these effects have been mostly disregarded in building science's comfort models.

As shown in Figure 2, building science's models of comfort have mostly ignored the non-physical characteristics of individuals and have only considered cognition implicitly and somehow reluctantly. These two components are what this thesis calls "the mind". Since cognition plays a relevant role in this theoretical framework, the following section will provide more details about it.

\subsubsection{Cognition and the relevance of intuition}

As suggested by Figure 2, cognition plays a relevant role in the theoretical framework of this thesis and thus this section introduces some concepts of cognition that will later shape the way this research is carried out. Great focus is put on formally defining the concept of Intuition.

By reviewing the literature, Stanovich and West (2000) found that several theorists in the discipline of psychology had proposed the coexistence of two different cognitive systems ${ }^{3}$.

\footnotetext{
${ }^{3}$ While Stanovich and West (2000) referred to these systems as "System 1" and "System 2", this dissertation adopted the easier-to-remember terminology used by Kahneman (Kahneman, 2003)
} 
The first one is called "intuition" and is mostly automatic, largely unconscious and quick. The second one is called "reasoning" and relates to the analytic intelligence that has more traditionally been studied (Kahneman, 2003; Stanovich \& West, 2000). An impression of the characteristics of both systems might be inferred from the fact that intuition is often said to be present in both humans and animals, while reasoning is supposed to be unique to humans (Evans, 2003).

While not explicitly expressed, intuition has been present in comfort research. For instance, Bluyssen (2009, p. 9) argues that people assess comfort both "knowingly (consciously) or unknowingly (unconsciously)". Likewise, Rohles (2007) found that people reported different thermal sensations when standing in two rooms at the same temperature but that looked different. He explains the results by stating that "Chamber B was an $8 \mathrm{ft}$ by $10 \mathrm{ft}(2.4 \mathrm{~m}$ by $3 \mathrm{~m})$ walk-in commercial refrigerator and anyone knows (recall the role of past experience and state of mind) that when you go into a refrigerator you feel cold"(2007, p. 22).

Rohles' (2007) experiment reveals how intuition seems to play a significant role in the development of comfort (i.e., through unconscious cognitive processes). Moreover, it seems to confirm the fact that intuitive judgments are difficult to control and modify (Kahneman, 2003) and that people might not know why they are making certain judgments (Kahneman \& Klein, 2009). For instance, people just felt colder and they did not necessarily know that the reason was related to how such a space looked.

But, even if they are often seen as unstable and unreliable, intuitive judgments are not necessarily always wrong (Bago \& De Neys, 2018). On the contrary, intuition can be trusted when trained through prolonged practice and feedback in environments in which there is a stable relationship between objectively identifiable cues and subsequent events (Kahneman \& Klein, 2009). These systems with stable cause-effect relationships (like most physical systems that people interact with) allow individuals to learn their governing rules. Consequently, since the built environment is governed by the highly stable laws of physics, it seems reasonable to assume that people are reasonably capable of intuitively predicting what will happen in the built environment, at least in the short term. For instance, Schweiker, Rissetto and Wagner (2020) found that people's thermal expectations are fulfilled the majority of the time, suggesting that people can read the cues on the environment to make relatively accurate inferences about it. This implies that the effect of cognitive processes on people's comfort is not chaotic. On the contrary, there might be a stable cause-effect relationship between comfort and people's cognition. 


\subsection{Research questions}

The theoretical framework developed in Section 3.1-graphically presented in Figure 2-reveals that building science's models of comfort have mostly ignored the Non-physical characteristics of individuals and have only considered Cognition implicitly and somehow reluctantly. These two components-cognitive processes and non-physical personal factors-are what this dissertation calls "the mind".

It should be said that some models and frameworks have incorporated cognition under the name of expectations (Luo et al., 2019), adaptability (de Dear, 2004; Nicol \& Humphreys, 2002), personal control (Luo et al., 2014; Veitch, 2001), and through the effect of elements of one domain into another (Chinazzo, Wienold, et al., 2018a; Golasi et al., 2019). However, these theoretical approaches often fail to provide a comprehensive list of the elements that make up "the mind" and their role in the determination of comfort. For instance, indices that try to predict people's overall satisfaction by considering more than one comfort domain (e.g., Buratti et al., 2018; Franzitta et al., 2014; Ricciardi \& Buratti, 2018) seem to rely on correlations and statistical analysis often lack an underlying model that reflects the causal relationship between variables (Schweiker, Ampatzi, et al., 2020). Based on this lack of understanding, this dissertation intends to answer the following questions: What are the main non-physical personal factors and cognitive processes that affect people's comfort? What is the role of these factors in the development of people's comfort?

Research question 1: What are the main non-physical personal factors and cognitive processes that affect people's comfort?

Research question 2: What is the role of the non-physical personal factors and the cognitive processes in the development of people's comfort?

It seems to be, however, that the reason building science has mostly disregarded "the mind" does not relate to a real lack of rigour, but pragmatism. In other words, comfort models need to be usable and incorporating "the mind" would imply dealing with the subjectivity and complexity that it carries. According to Reinhart (2014, p. 27), building scientists are afraid of leaving too much room for subjectivity.

As simplified representations of the concept of comfort, quantitative models of comfort will undoubtedly disregard some relevant factors and therefore they cannot be perfectly accurate. Building science understands this. For instance, thermal comfort models 
acknowledge that "due to individual differences it is impossible to specify a thermal environment that will satisfy everybody... There will always be a percentage of dissatisfied occupants" (ISO, 1984, p. 4). However, "it is possible to specify environments known to be acceptable by a certain percentage of the occupants." (ISO, 1984, p. 4). In other words, quantitative models of comfort attribute their errors to the existence of unobserved or disregarded factors that affect people's comfort.

Building science's approach is far from unique, as other disciplines that approach human behaviour and cognition from a quantitative tradition are also aware of the factors they disregard and thus present their results in terms of probabilities. For instance, Train (2002, p. 3) states that, since some factors that affect people's choices are unobserved, these are "not deterministic and cannot be predicted exactly. Instead, the probability of any particular outcome is derived". However, contrary to the discrete choice models presented by Train, utilizing this approach in comfort research does imply a contradiction because, after the great emphasis given to "subjectivity" and "the mind" (see Section 2.1), comfort models in building science seem to suggest that "the subject" and "the mind" can be ignored (i.e., treated as unobserved) without great loss of predictive capacity. Thus, this thesis also intends to answer the following question: How can predictive models of comfort incorporate people's non-physical characteristics and their cognitive processes?

Research question 3: How can predictive models of comfort incorporate people's non-physical characteristics and their cognitive processes?

\subsection{Conclusions}

This chapter drew on the literature of several disciplines to develop an understanding of how people's comfort develops, and a language utilized in the rest of this dissertation. A key insight derived from this chapter is that people's cognition and their non-physical personal factors-i.e., what this dissertation calls "the mind"-are known to affect the development of people's Comfort, but they still tend to be disregarded by building scientists. This is considered a contradiction that this research aims to mitigate by exploring the "the mind" in the context of people's comfort and by proposing ways in which it can be better incorporated into building science's research and practice. Exploring "the mind" often implies using qualitative and exploratory research. The following chapter explains the research design in detail. 


\section{METHODOLOGY}

"It's the only mind we've got, and we have no choice but to take its intuitions seriously"

SteVEn Pinker, THE Blank SLate

This research aimed to explore the "the mind" in the context of people's comfort and to propose ways in which it can be better incorporated into building science's research and practice. As explained in Section 2.1, this goal was justified by the fact that even if building science knows that comfort is a state of the mind, its comfort research tries to avoid "the mind" as much as possible. Section 2.3 argued that building science seems to disregard "the mind" to avoid the intrinsic complexity of subjectivity, which cannot be appropriately studied through the (mostly quantitative and confirmatory) methods that building science has historically utilized for studying comfort. This suggests that this research—which attempted to explore the elements traditionally disregarded by this disciplinetraditional building science methodologies. This chapter provides details on how this research was conducted and explains the rationale behind the methodology, methods, and paradigm utilized.

\subsection{Research paradigm}

Section 2.4.1 argued that building science performs comfort research mostly through quantitative and confirmatory methods. However, Sections 2.4.2 and 2.4.3 advocated for a more qualitative and exploratory approach to comfort research, and to embrace "the mind" in a much more explicit manner. These two changes - in the methods utilized and in the conceptual frameworks used to design research and to interpret its results-can be seen as a proposition to change the paradigm through which building science's comfort research is performed. This section explains the paradigm this research utilized.

The concept of paradigm, as understood within science and research, was popularized by Kuhn (2012) in his book "The Structure of Scientific Revolutions". In that book, the concept is specifically defined in two main different ways, which will shape the structure of this section: 
"On the one hand, it stands for the entire constellation of beliefs, values, techniques, and so on shared by the members of a given community. On the other, it denotes one sort of element in that constellation, the concrete puzzlesolutions which, employed as models or examples, can replace explicit rules as a basis for the solution of the remaining puzzles of normal science"

(Kuhn, 2012, p. 174)

The first connotation of the word paradigm defines it as a set of virtually unquestioned assumptions that scientists of a certain discipline share. On its second connotation, the word paradigm is an exemplar or a remarkably good and instructive example (Hacking, 2012) that serves as guidance for the performance of the so-called normal science. That is to say, the kind of research that is "firmly based upon one or more past scientific achievements, achievements that some particular scientific community acknowledges for a time as supplying the foundation for its further practice" (Kuhn, 2012, p. 10). Typical exemplars are “Aristotle's Physica, Ptolemy's Almagest, Newton's Principia and Opticks, Franklin's Electricity, Lavoisier's Chemistry, and Lyell's Geology... [which] served... to define the legitimate problems and methods of a research field for succeeding generations of practitioners" (Kuhn, 2012, p. 10). In simple terms, the paradigm that a set of researchers adhere to not only defines the beliefs that exist behind their work but also which methods these researchers will use and how they utilize such methods.

Within the different scientific disciplines, research paradigms are often organized in a continuum that lies between the two opposite ends: those that use qualitative methods and those that use quantitative methods (Morgan, 2007). The two ends of this continuum can be seen as-after an oversimplification made in favour of clarity-paradigms that "approach research through words only" and those that do so "through numbers only", respectively. Within this continuum, researchers may position themselves closer to one end or the other, or in the middle. In the case of the latter, such researchers would often adhere to a third paradigm, the mixed methods approach (Creswell, 2014).

This thesis could neither use "words only" nor "numbers only". It seemed rather inappropriate to use purely numerical methods to understand what people expect from-or understand by - "a comfortable home", considering that comfort is often associated with the highly qualitative concepts of wellbeing, pleasure and happiness (Altomonte et al., 2020; Gagge et al., 1967). Similarly, it was considered to be quite hard only to use words to solve the equations that represent the physical state of the dwelling that produces the stimuli that people might interpret as (dis)comfort. Therefore, this research stood in the middle of the qualitative/quantitative spectrum. It is worth mentioning that, even if some textbooks 
associate this position with the use of a mixed-methods methodology (Creswell, 2014; Morgan, 2014), this dissertation gathered data only through qualitative ones. The reason for this is that qualitative methods were considered the most appropriate ones for answering the research questions formulated in this dissertation. The reasons for this are described in Section 4.3.

This attitude towards research—using "what works" for each task—is often associated with a research paradigm called pragmatism (Kivunja \& Kuyini, 2017; Morgan, 2014; Parvaiz, Mufti, \& Wahab, 2016), which is the research paradigm in which this researched dwelled. Nevertheless, a paradigm not only defines the set of methods researchers use but also a set of beliefs they hold. This means that simply stating what kind of methods were used is not enough. The following paragraphs explain this thesis's underlying beliefs, both related to the nature of being (i.e., ontology) and to how can we gather knowledge about these beings (i.e., epistemology).

According to Creswell (2014), pragmatism is not strictly committed to a particular philosophical system, which implies that researchers have the freedom to choose their position in the qualitative/quantitative spectrum. In the case of this thesis, the approach was driven by the description of pragmatism provided by Morgan (2014), which emphasises the thought of Dewey (1965). As these sources do, this research understood that reality exists regardless of humans, and thus human experiences are constrained to the nature of this reality (Morgan, 2014). People's understanding of the world, on its part, is limited to the interpretations of their experiences within the world (Morgan, 2014). Thus, different people will have a different perception of the world based on their current and past experiences, but it is not possible to say that every possible interpretation of the world is equally reasonable. Some of them will simply not be consistent with the nature of the world, which is why it is important to ground all knowledge in observations. As stated by Dewey (1965, p. 21), "theory may intervene in a long course of reasoning, many portions of which are remote from what is directly experienced. But the vine of pendant theory is attached at both ends to the pillars of observed subject-matter".

This "observed subject-matter" over which theory is supported is not necessarily constrained to physical objects and measurable quantities. On the contrary, nature includes anything that can be experienced, such as aesthetic and moral traits (Dewey, 1965, p. 25). Acknowledging this helps to understand why the means for acquiring knowledge cannot be categorically limited to quantitative or qualitative. The methods to use should be those deemed more appropriate for studying the investigated phenomenon (Kivunja \& Kuyini, 
2017). Instruments and sensors are very successful at gathering quantifiable and measurable data, while interviews may help to acquire information on experiences, perceptions and other more abstract elements of nature.

It was mentioned earlier that, according to Dewey (1965, p. 21), "theory may intervene in a long course of reasoning, many portions of which are remote from what is directly experienced. But the vine of pendant theory is attached at both ends to the pillars of observed subject-matter". This statement suggests two things. First, whatever theory is utilized needs to match what is experienced (i.e., to match the facts). And second, that the utilized theories and models may include counterintuitive portions that seem incorrect and even absurd. In those cases, "the most useful thing to do... is not to criticize its assumptions but rather to test its theorems. If the theorems fit the data, then the theory has at least heuristic merit" (Edwards, 1954, p. 382).

However, researchers will often find the situation in which no existing theory "fits the data" well enough. In such a case, Kuhn (2012, p. 146) suggests that "it makes a great deal of sense to ask which of two actual and competing theories fits the facts better" because, as a matter of fact, "all historically significant theories have agreed with the facts, but only more or less" (2012, p. 146). Of course, the researcher will need to decide, based on his or her own experience, whether the disagreement between a certain theory and the observed facts is small enough for such a theory to have "at least heuristic merit". In such a situation, the researcher must try to anticipate how others will judge such decisions and choices from their own experience (Morgan, 2014). For instance, through a rigorous peer-reviewing process.

In addition to their accuracy, the research documented in this dissertation also emphasized the fact that the researcher must evaluate whether the models or theories utilized are useful in the context of the specific research or application. This implies not only understanding whether all the relevant concepts and phenomena being studied are incorporated in such models and theories, but also whether they are simple and mature enough to serve the purpose of the discipline that will use them. For instance, the model of the Economic Man has been historically a very valuable model for economists despite being highly simplistic. According to Thaler (2018a)—whose work has focused on replacing the Economic Man with a less simplistic model of humans — such a model became a standard mainly because it was usable. It served the purpose of allowing scientists to create theories and theorems about phenomena that would be otherwise impossibly complex. Kahneman (2003, p. 1449) expresses this by positing that "economists often criticize psychological research for its propensity to generate lists of errors and biases, and for its failure to offer a 
coherent alternative to the rational-agent model". This suggests that the arguably more realistic models originating in Psychology were not useful in an Economic Science context because they could not deliver what Economists were looking for when using them. Something similar may be occurring in the case of building science and comfort because, as explained in Section 2.3, the usability of the models is often favoured over their comprehensiveness. While this does not mean that these models are completely wrongbecause they can still have heuristic merit in some situations-it significantly limits their generalizability. For instance, comfort models that do not incorporate psychological factors are not likely to be accurate in situations when psychology plays a dominant role.

Oversimplification, however, is the other end, and the research approach of this thesis intended to avoid it. Kahneman (2003, p. 1449) agrees with this position when stating that the Economists' argument against the complex models grounded in psychology "is only partly justified: psychological theories of intuitive thinking cannot match the elegance and precision of formal normative models of belief and choice, but this is just another way of saying that rational models are psychologically unrealistic". Thus, in the pursuit of elegance and simplicity, predictions of models and theories can dramatically deviate from the observations. For instance, assuming that all consumers are perfectly informed may help simplify models, but sometimes at the cost of a profound effect on the nature of the market equilibriums (Akerlof, 1970; Rothschild \& Stiglitz, 1976; Stiglitz, 2002). Thus, this assumption needs to be rejected not because it looks counterintuitive, but because it may cause the model's predictions to critically deviate from the observed facts.

In conclusion, this dissertation does not reject qualitative research approaches. On the contrary, the lack of precision of qualitative data-sometimes feared by quantitative researchers - can become a strength at the time of investigating intrinsically imprecise topics, such as comfort. In the same way, this dissertation does not overlook the elegance, precision, and predictive capacity of quantitative models. However, it understands these models are simplified representations of a much more complex - and often vague and imprecisereality. Consequently, a proper understanding of reality must precede the development of quantitative models.

\subsection{Research methodology}

Section 4.1 argued that, according to Kuhn (2012), a research paradigm not only defines the beliefs that exist behind scientists' work but also which methods they will use and how they utilize them. In the specific case of building science, "research on occupant comfortbe it thermal, visual, or acoustic - is typically performed in either a controlled laboratory 
environment or in situ" (O'Brien et al., 2019, p. Introduction). Within these two settings, non-experts are asked to fill questionnaires that are simple enough for the answers to be treated as objective data (Schweiker et al., 2017). Building scientists apparently justify this tradition based on the belief that non-expert's opinions are unreliable. For example, Da Yan (2018, p. 13) argues that "self-reported behavior may not always match observed behavior" (as confirmed by Tamas, O’Brien and Gunay, 2018). Similarly, O’Brien et al. (2019, p. Introduction) explain that "regardless of the accuracy of self-reporting in surveys, participants may not have the insight or knowledge required to identify the subtle building characteristics that are known by researchers to influence comfort". Thus, building science's comfort research tends to utilize approaches that are mostly quantitative and confirmatory. These approaches are quantitative because they often rely on correlations, mathematical models, and/or statistical tests (Schweiker, Ampatzi, et al., 2020); and they are confirmatory because these analyses use data gathered from a pre-defined list of relevant elements (e.g., Haldi \& Robinson, 2010).

Within the thermal domain, for instance, people are regularly asked about how they feel right here and right now through a simple bipolar adjectives item that goes from 'hot' to 'cold' going through 'neutral' (de Dear et al., 2018; Schweiker et al., 2017; Song et al., 2018). The reason for asking this question is simple: there is no way of acquiring (i.e., measuring) such information (Luo, 2020, p. 7). This self-reported subjective perception is known as thermal sensation and has the advantage of being "a rational experience that can be described as being directed towards an objective world" (Hensen, 1991, p. 2.4). In other words, even if it is self-reported-therefore, subjective-the question leaves so little room for people's opinion that its response can be treated as an objective value (e.g., Djongyang et al., 2010; Schweiker et al., 2017). This is the case for both the rational (Auliciems \& Szokolay, 1997; Cheung et al., 2019) and adaptive (Brager \& de Dear, 1998; Humphreys et al., 2016; Nicol et al., 2012) approaches to thermal comfort. The same procedures are present in research about the daylighting domain. For instance, research about glare (Konstantzos \& Tzempelikos, 2017; Sarey Khanie et al., 2017; Wienold et al., 2019) and even studies about more qualitative constructs (Cauwerts \& Bodart, 2011; Chamilothori et al., 2019; Newsham et al., 2005; Rockcastle et al., 2017) tend to use Likert-scale questions for gathering data with which to test mathematical models and equations.

While they have proved useful in many cases, these confirmatory approaches to research were not appropriate to answer the questions that drove this research. The reason for this was that trying to understand — with very little previous knowledge — what are the main nonphysical personal factors and cognitive processes affecting people's comfort (i.e., Research 
Question 1) and the role they play (i.e., Research Question 2) is not a confirmatory process, but an exploratory one. Furthermore, since the phenomenon studied in this research-the development of people's Feeling of Comfort-is highly qualitative, the method to use not only had to be exploratory but qualitative. Then, only after developing a good understanding of the phenomenon being studied, it was deemed appropriate to enter into the quantitative stage of this research (i.e., Research Question 3).

Exploratory research is often performed in two stages, the first one often being qualitative (Creswell, 2014). For example, it is possible to start with a round of interviews that allow eliciting a population's commonly held beliefs and attitudes associated with a certain behaviour (Francis et al., 2004), and then translate these findings into a questionnaire to perform quantitative analysis of a formal theory (Ajzen, 1985, 1988, 1991). As explained in the next section (4.3), this research utilized a similar strategy.

The practice of asking open-ended questions to non-experts-i.e., to gather qualitative data from non-experts-is uncommon within building science, although not inexistent. For instance, Attia (2020) used in-depth interviews to investigate the effect of people's behaviour on residential energy savings after retrofitting dwellings. Similarly, Bend and Sunikka-Blank (2015) used semi-structured interviews to understand the variety and complexity of people's heating behaviour. Qualitative data is especially useful when attempting to understand what and how people are thinking in different situations. Partly because of these benefits, gathering non-experts' responses to open-ended questions is routine for other disciplines (Fontana \& Frey, 2005; Johnstone, 2017; Trocchia et al., 2007). For instance, interviews have been useful for understanding what people know and how they reason, and which environmental cues they notice when making decisions (Crandall \& Hoffman, 2013). Similarly, in-depth interviews have proved useful for understanding people's perceptions of quality and value (Zeithaml, 1988); and focus groups, for explaining why people's behaviour does not match their attitudes and self-reported behaviour (Johnstone \& Tan, 2015). In summary, qualitative research has proved useful for investigating different aspects of "the mind". Consequently, since this research focused on "the mind" (as concluded by Chapter 3), its approach was mainly qualitative.

\subsection{Research strategy}

Chapter 3 introduced the three questions that this research answered:

1. What are the main non-physical personal factors and cognitive processes that affect people's comfort? 
2. What is the role of non-physical personal factors and cognitive processes in the development of people's comfort?

3. How can predictive models of comfort incorporate people's non-physical characteristics and their cognitive processes?

From a research design perspective, these three questions can be interpreted as (1) what are the variables that explain people's comfort, (2) how these variables relate to each other, and (3) how this information can be operationalized. This reinterpretation helped to design a two-stage research strategy. First, an exploration of the concept of comfort helped answer research questions 1 and 2 by helping to develop an understanding of comfort which is not constrained by the need for usability or for being quantifiable. Then, the second stage operationalized this understanding of comfort by modelling it mathematically and by performing building performance simulations that take this into account. Figure 3 shows this process graphically.

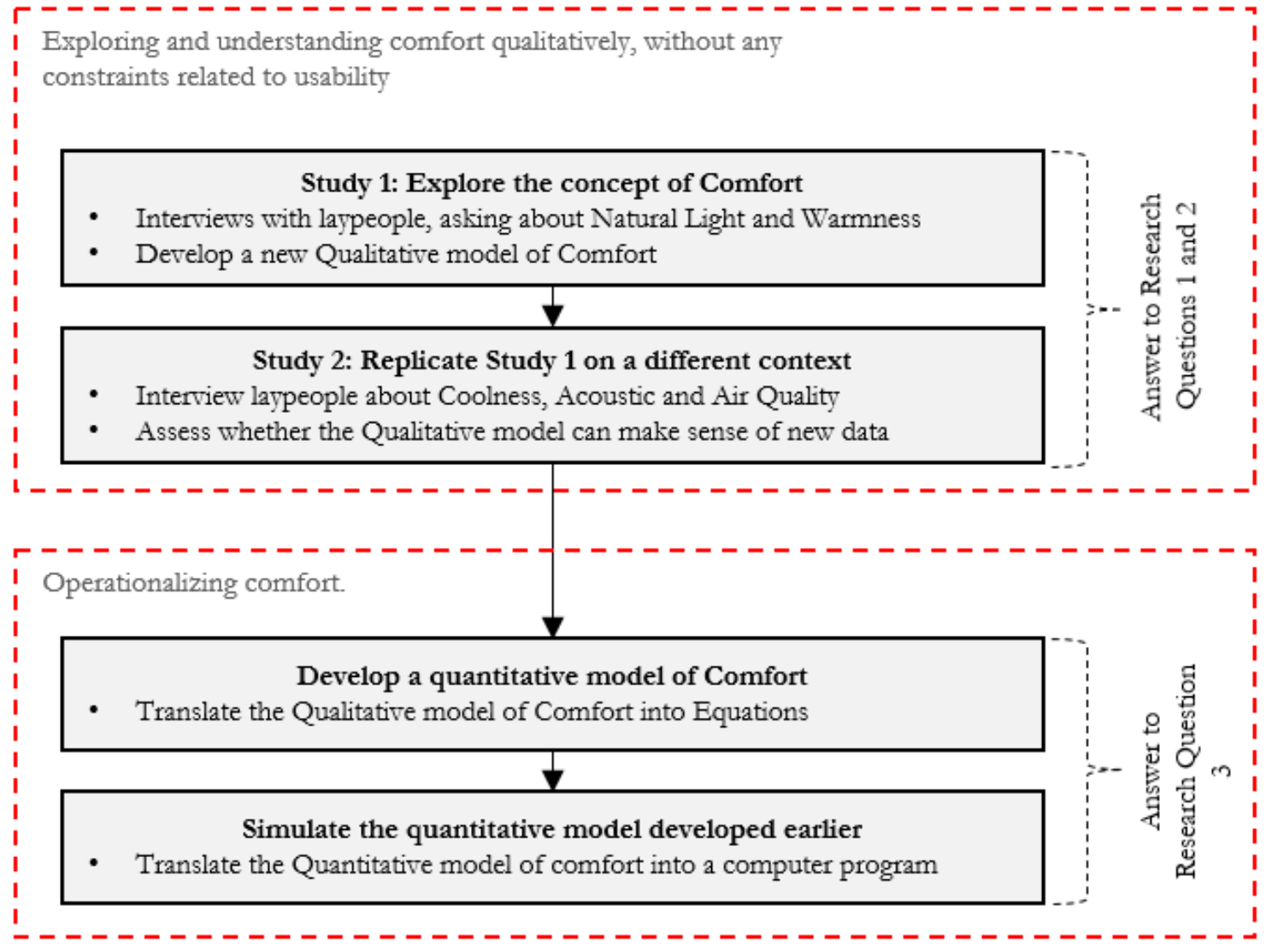

Figure 3: Strategy utilized in this research

The first stage of this research — in which research questions 1 and 2 were answeredwas carried out through two studies. Both studies involved gathering data through interviews in which respondents-i.e., people from Chile and New Zealand with no expertise in 
building science or other related disciplines-described a comfortable home in their own words. The difference between the two studies are the sub-areas of comfort they focused on, and the purpose of the data they produced within this research. Specifically, people interviewed in Study 1 described "a warm dwelling" and "a dwelling with good daylight", and the data was utilized to develop a qualitative model of comfort. Study 2 was focused on "coolness", "acoustic comfort" and "air quality", and the data it produced was utilized to see whether the qualitative model developed earlier was also capable of making sense of this new data.

It should be noted that, when combined, the two studies performed in stage 1 of this research investigated the four main elements of indoor environmental quality; that is to say, thermal, visual and acoustic comfort, and air quality (Andargie et al., 2019). Thermal comfort, however, was separated into coolness and warmness for two reasons. First, to relax the assumption that high and low temperatures are equally pleasant/unpleasant; and second, because people who live in locations with different climates are likely to be much more familiar with one than the other. For instance, people who live in colder areas were not expected to be familiar with the issue of temperatures being too high and thus their descriptions of "a thermally comfortable home" would overemphasize one side of it.

After developing an understanding of what comfort is, how it develops, and what the factors and cognitive processes influence it, this research entered a second stage. The purpose of this second stage was to operationalize the understanding of comfort developed in the first stage and, in the process, to answer research question 3. This stage was based purely on the qualitative model of comfort developed in stage 1 and thus it did not involve gathering new data. On the contrary, this stage involved developing a mathematical model of comfort and then prototyping a building performance simulation tool that could incorporate it.

\subsection{The method for collecting data}

As suggested in Section 3.2, the questions that this research intended to answer require exploratory and qualitative data gathering techniques. Based on this requirement, the method used was a semi-structured interview. The advantage of interviews is that they allow documenting the different visions that people have of the same situation or topic Johnstone, 2017). This characteristic was particularly appropriate for the topic of this research; i.e., comfort as a subjective state of the mind.

According to Fontana and Frey (2005, p. 697), "interviewing is one of the most common and powerful ways in which we try to understand our fellow humans" and the interview can 
have different forms. For instance, structured interviews involve asking the same questions to all respondents and constraining their answers to a predefined set of options (Fontana \& Frey, 2005). This approach, however, was not appropriate for this research because, at the time of gathering data, it was not clear which factors of "the mind" is relevant in the determination of comfort and thus it was impossible to compile a list of potential answers. Another form of interview is the unstructured one, where there are no predefined questions and the respondent becomes the driver of the conversation (Johnstone, 2017). This format was not appropriate for this topic either, as the purpose was to gather data related to a specific topic, which is comfort. Thus, a combination of the two was used: semi-structured interviews where a set of questions is predefined but new questions may be asked when the interviewer finds it necessary (Johnstone, 2017). This format fulfils the requirement of being exploratory because the open-ended nature of the questions asked allows for the confirmation or contradiction of what researchers already know about comfort. Also, semi-structured interviews were particularly appropriate for this research because they can gather data about the cues and contextual factors that drive people's judgments and decisions (Crandall \& Hoffman, 2013; Kahneman \& Klein, 2009).

The following section introduces a semi-structured interview designed to elicit factors that, according to the respondents, are relevant in the determination of comfort. This same interview was used in both stages of data collection, explained in Section 4.3.

\subsubsection{Interview design and general strategy}

People today spend most of their lives in or around buildings. This implies that, at a certain age, they will have occupied a great number of dwellings, hotels, workplaces, and other examples of the built environment. By adulthood, people will probably know that some materials feel warmer than others when touching them, and even that some windows are more likely to cause condensation. In other words, this extensive use of buildings will have probably trained adults' intuitive systems (Kahneman \& Klein, 2009), allowing them to unconsciously read cues from the environment to make inferences about the quality of the built environment. When people get into a room, they have the impression of being or simply feel comfortable or uncomfortable.

Intuition allows people to make unconscious judgments of spaces based on multiple environmental cues. For instance, it is possible that the reason why colours of light affect people's thermal sensations-predominantly through psychological processes (Chinazzo, Wienold, et al., 2018a, 2018b; Golasi et al., 2019)—is related to people's intuition and the history that has trained it. Similarly, since "anyone knows... that when you go into a 
refrigerator you feel cold" (Rohles, 2007, p. 22), a room that looks like a refrigerator actually feels cold. Likewise, since everyone knows that certain temperatures feel cold, knowing the temperature of a room affects people's thermal sensation (Rohles, 2007).

The design of this interview intends to exploit people's trained intuition about the built environment by asking them to describe-in their own words-a dwelling that was particularly comfortable in a specific domain (i.e., warmness, coolness, acoustic, air quality, daylight). An informal tone was encouraged to prevent respondents from overthinking their answers, which might stop them from expressing their intuitive impressions. Within their description of a comfortable home, respondents were asked to express both physical features that make a dwelling comfortable (e.g., "dwellings with good natural light have big windows") as well as what they think their quality of life would be in it (e.g., "a dwelling with good natural light looks better"). Such a description contains information about what people mean by comfort, including the factors and cognitive processes that are relevant in the development of comfort.

While respondents were often aware of certain causal relationships, this interview purposefully did not discuss those topics. The reason for this is that, while respondents might have appropriately identified which features cause which effects (because of their highly trained intuition) (Kahneman \& Klein, 2009), they were specifically chosen not to have what is known as "book knowledge" (Crandall \& Hoffman, 2013); i.e., the knowledge about the theory and models behind building physics. Without this knowledge, respondents do not know how or why something happens, even if they are sure that they know it (Crandall \& Hoffman, 2013). Moreover, if respondents start to think about why or bow something happens, they may doubt their intuition and even avoid saying some features they think are important. Therefore, understanding and verifying the existence of the causal relationships suggested by respondents is the task of researchers.

A final consideration comes from the fact that the concepts retrieved from memory are not often the most relevant or reliable, but simply the most easily retrievable (Wyer, 2008). For this particular reason, this research prioritized people who had recently gone through a home-searching process (less than 3 years). This ensured that respondents had a fresh memory of their expectations and the features they use for assessing how comfortable dwellings were.

In any case, researchers should not expect respondents to provide a complete list of all the relevant factors that affect comfort along with their consequences. On the contrary, respondents are very likely to provide information that is not necessarily relevant, and also 
to fail to provide information considered relevant by themselves. Thus, gathering a comprehensive set of factors implies interviewing enough people to reach theoretical saturation. That is, to reach the point at which performing more interviews does not provide more information (Bowen, 2008; Corbin \& Strauss, 2008; Francis et al., 2010; Rowlands, Waddell, \& McKenna, 2015).

The interview guide_-detailing the procedure and structure-is available in Appendix I.

\subsection{Data collection procedure}

As explained in Section 4.3, data was collected in two stages. The first one involved interviewing 18 people (the first interview being on the 27 of June 2019 and the last one on the 6 of August 2019) and the second one 24 people (the first interview being on the 28 of April of 2020 and the last one on the 20 of October 2020). Respondents were interviewed one by one, either in person (in a public place) or remotely (through phone or Zoom or other). Interviews generally lasted between 25 to 40 minutes. The medium used for the interview-face to face, phone or audio-visual means of remote communication-did not seem to make any difference. Likewise, even if the second stage of interviews took place in periods of Covid-19 lockdown, nothing in people's responses suggest that the pandemic affected the data gathered in any significant way. (One of the conclusions of this research is that people seem to be good at distinguishing between temporal factors that affect their comfort-e.g., constructions, heat waves, pandemics-and permanent factors associated with "normal" situations—e.g., cold winters, noisy neighbours.)

Both stages utilized the same interview-introduced in Section 4.4.1, and explained in detail in Appendix I-but focused on different domains of comfort. Specifically, respondents of the first stage were asked to "describe a warm home" and later to "describe a home with good natural light". In contrast, respondents of the second stage were first asked to "describe a cool dwelling", then a "dwelling with good air quality", and finally "a dwelling with a good acoustic performance". Respondents from Chile and New Zealand (predominately from the cities of Santiago and Wellington, respectively) were recruited for both stages, and the interviews were performed either in Spanish or English, depending on the first language of the respondent. The interviews were translated by the researcher, who speaks both Spanish and English. The translation aimed to keep a description of what, according to the respondent, comfortable situations are. For example, the term "fresh air" in Chilean Spanish does not necessarily mean "clean air" but "cool/cold air". The translation, then, focused not on the cleanliness of the air but its relative temperature. As will be explained in more detail later in this section, a copy of the outcome of each interview was 
sent to every respondent for them to check.

Recruiting implied sending the respondents a project information sheet outlining the research aims and objectives as well as interview requirements (see Appendix II), and a form intended to obtain informed consent (see Appendix III). They had to agree with these documents before the interview. People were recruited through direct contact with, social media posts asking for volunteers, and a snowball effect with multiple starting points (i.e., friends and respondents helped by recruiting people their contacts). The value of using multiple starting points is that it reduces the selection bias (Atkinson, Flint, \& Gilbert, 2001), meaning that it helps recruit respondents who are not socially connected. The details of the sample in each stage are available in Section 4.6. Respondents were not given any incentives to participate in this research.

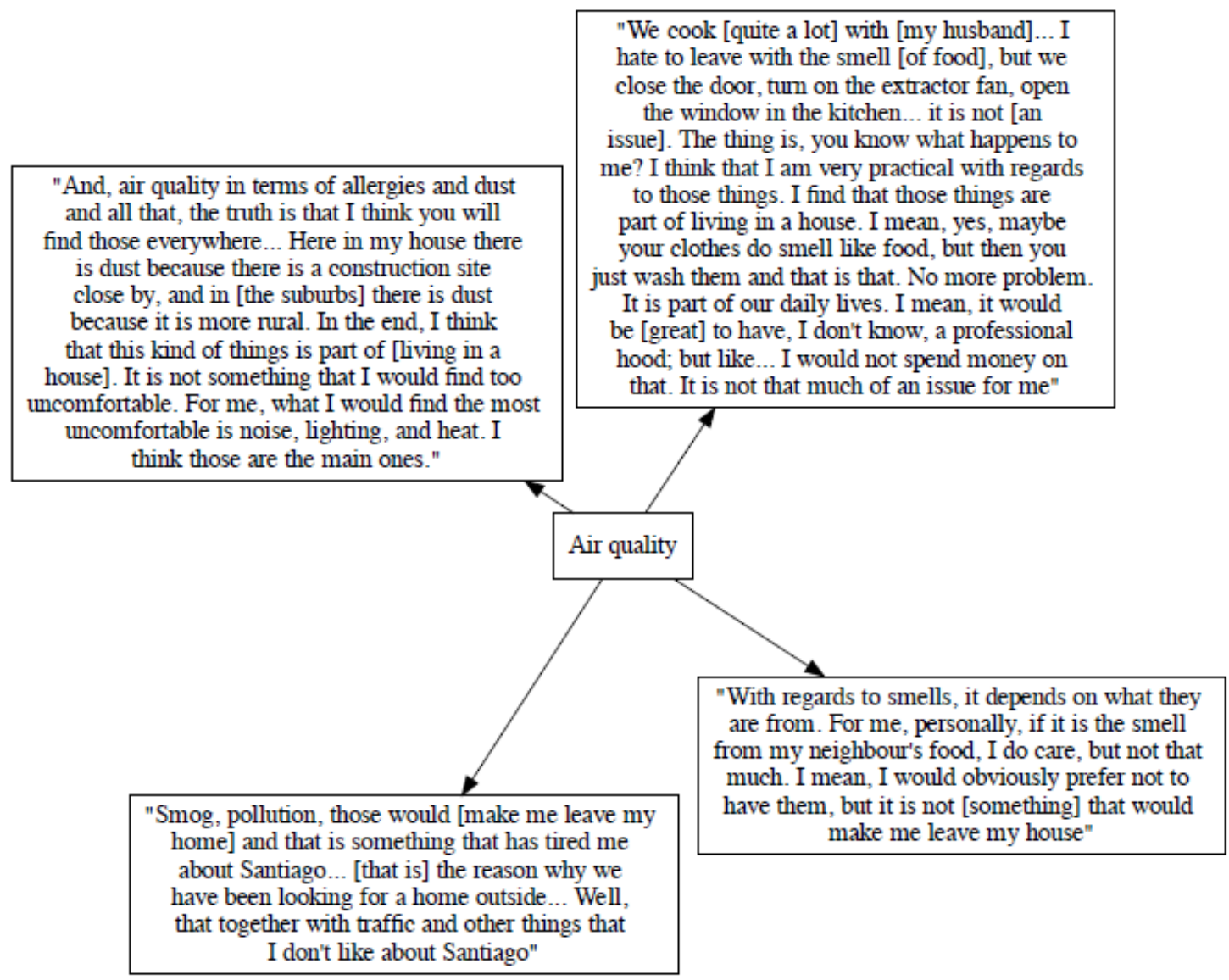

Figure 4: A small example of the mind map that resulted from an interview about air quality

Even if the topic of the interview was not likely to generate any controversy, respondents were assured that all the conversations were confidential. Also, the conversations were recorded, but such records were only used for analysing the data and never published. On the contrary, the data gathered from the interview was reduced to an anonymised mind map (see an example in Figure 4) and uploaded to a permanent online repository. 
The main outcome of each interview was a mind map relating housing attributes that may be physical features or more experiential ones (e.g., what the dwelling affords to whoever lives in it). Inspired by the Meaning Structure method (Coolen, 2008), such a structure was to be built during the interview. Even more, if the interview was carried face-to-face, respondents were welcome to comment, participate, and make amendments to what the interviewer is writing down. This was seen as beneficial for four key reasons. First, the respondents get to check the data during the interview. Second, because the structure allows to easily find gaps and contradictions in the information, thus highlighting areas that need to be further questioned. Thirdly, because these structures do not contain too much irrelevant information (informal conversations often involve stories and anecdotes), thus they could be digitalized relatively fast and therefore allowed to gather a larger sample. And fourth, this form of representation is convenient because people's description of a comfortable home was not always linear, and therefore having a non-linear concept structure (as opposed to a chronologically ordered text) makes the data easier to read and analyse. After the interview was finished, the concepts in the mind map were replaced with their corresponding quotes. This effectively became a pseudo-transcription that allowed for the communication of the results in a much more expressive way. Figure 4 shows an example of the result.

Even if respondents who participated face to face were welcome to participate in the development of the mind map during the interview, this did not happen often (although the notes were still taken openly and transparently). This implies that the respondents did not rigorously check the data being gathered during the interview. To ensure reliability, the data—after being pseudo-transcribed—was sent to the respondents for them to check and propose any changes. Details about these checks are provided in Section 4.8.

\subsection{Sample}

The target population of this study included people who complied with three main requirements:

1. Having searched for a home relatively recently or being currently searching for one.

2. Having done it for living in it themselves

3. Having performed their search in Chile or New Zealand.

It is known that this target population is not representative of all people belonging to all demographic segments and cultures within their respective countries. For instance, people who intend to purchase homes probably have an economic status that does not illustrate the 
whole population of Chile or New Zealand. Interviewing people from other demographic, economic, and cultural backgrounds is proposed as future research. In a similar manner, since this research targeted residential settings-i.e., people searching for homes were asked to describe comfortable homes - the results of this research might not be directly applicable to other settings. For instance, it is unclear whether the models presented in this dissertation also represent the way people think about comfort in office buildings or even in airports, train stations, supermarkets, etc.

Table 1: Respondents who participated in Study 1

\begin{tabular}{|c|c|c|c|c|c|}
\hline ID & Country of search & Age & Gender & Marital status & Has children? \\
\hline 1 & Chile & 41 & Male & Married & Yes \\
\hline 2 & Chile & 34 & Male & Married & Yes \\
\hline 3 & Chile & 36 & Female & Single & No \\
\hline 4 & Chile & 33 & Male & Married & Yes \\
\hline 5 & Chile & 32 & Male & Married & No \\
\hline 6 & Chile & 37 & Female & Single & No \\
\hline 7 & Chile & 29 & Male & Single & No \\
\hline 8 & Chile & 40 & Female & Single & No \\
\hline 9 & Chile & 29 & Male & Single & No \\
\hline 10 & New Zealand & 28 & Male & Single & No \\
\hline 11 & New Zealand & 61 & Female & Single & No \\
\hline 12 & New Zealand & 29 & Female & Married & No \\
\hline 13 & New Zealand & 33 & Male & Married & No \\
\hline 14 & New Zealand & 29 & Male & Single & No \\
\hline 15 & New Zealand & 38 & Male & De Facto & No \\
\hline 16 & New Zealand & 27 & Female & Single & No \\
\hline 17 & New Zealand & 29 & Female & Engaged & No \\
\hline 18 & New Zealand & 66 & Female & Widow & No \\
\hline
\end{tabular}

The first requirement for the target population was defined based on the argument in Section 4.4.1. Specifically, on the need for respondents to have a fresh memory of the 
expectation they had about a comfortable home and the cues they used to make judgments about it. The second requirement relates to the differences in motivations and the reasons for searching that different people have. People who are searching as an investment or on behalf of someone else might have different motivations and thus their expectations will be different. Finally, the reason for focusing on Chile and New Zealand is that they are significantly different from each other and yet they are easy to reach by the researcher-who is Chilean currently living in New Zealand. Examples of differences between New Zealand and Chile are the population and density of their cities, per capita income, climate, and culture, among others. The existence of these differences counteracts some of the representativeness issues mentioned earlier because, even if the target population is not representative of all the people in each country, putting together the target populations of Chile and New Zealand significantly increases the diversity within the sample. The existence of this cross-cultural diversity suggests, for instance, that the results might be replicated in a third country or culture, or even in other population segments within these two countries. Again, verifying this is proposed as future research.

To capture as many different views about comfort as possible within the target population, this research tried to maximize the diversity of the participants. Nevertheless, the nature of this study made it difficult to define what diversity truly means. The reason for this difficulty is that this was an exploratory investigation that, among other objectives, intended to determine some of the non-physical personal factors and cognitive processes that affect people's comfort (i.e., research question 1). Thus, defining beforehand which attributes should be varied was impossible.

Likewise, focusing on participants who had searched for a new home relatively recently (the threshold utilized was three years ago) made it difficult to know whether the sample was representative of the target population. For instance, it is known that not all age groups are equally likely to search for a new home (Clark, 2013; Clark \& Lisowski, 2018). Consequently, it is hard to say whether the fact that the interviewees of this research tend to be relatively young corresponds to recruitment biases or to the natural demographics of the target population (i.e., of the people who search homes for living themselves) ${ }^{4}$.

\footnotetext{
${ }^{4}$ Note that this discussion is about whether the respondents of this research were representative of the target population and not whether the results of this research could be generalized to the whole target population. In fact, the purpose of the interviews performed during this research was not to produce generalizable results but simply to gather the different views about comfort that people have.
} 
Table 2: Respondents who participated in Study 2

\begin{tabular}{|c|c|c|c|c|c|}
\hline ID & Country of search & Age & Gender & Marital status & Has children? \\
\hline 1 & Chile & 32 & Male & Married & Yes \\
\hline 2 & Chile & 34 & Male & Married & Yes \\
\hline 3 & Chile & 33 & Male & Married & Yes \\
\hline 4 & Chile & 27 & Female & Married & Yes \\
\hline 5 & Chile & 30 & Male & Married & No \\
\hline 6 & Chile & 31 & Male & Single & No \\
\hline 7 & Chile & 30 & Male & Married & Yes \\
\hline 8 & Chile & 31 & Female & Married & Yes \\
\hline 9 & Chile & 32 & Male & Married & Yes \\
\hline 10 & Chile & 30 & Female & Married & Yes \\
\hline 11 & Chile & 30 & Female & Married & Yes \\
\hline 12 & Chile & 32 & Female & Married & Yes \\
\hline 13 & Chile & 26 & Female & Married & No \\
\hline 14 & New Zealand & 42 & Male & Married & Yes \\
\hline 15 & New Zealand & 42 & Female & Married & Yes \\
\hline 16 & New Zealand & 26 & Female & In a relationship & No \\
\hline 17 & New Zealand & 59 & Female & Married & Yes \\
\hline 18 & New Zealand & 28 & Female & In a relationship & No \\
\hline 19 & New Zealand & 36 & Female & Divorced & No \\
\hline 20 & New Zealand & 36 & Female & Single & No \\
\hline 21 & New Zealand & 34 & Male & Married & Yes \\
\hline 22 & New Zealand & 27 & Male & Engaged & No \\
\hline 23 & New Zealand & 42 & Female & De facto relationship & Yes \\
\hline 24 & New Zealand & 25 & Female & In a relationship & $\mathrm{No}$ \\
\hline
\end{tabular}


Despite the difficulty in defining what diversity is in terms of demographic or personal traits, the sampling process did try to maintain diversity in characteristics such as gender, age, and country of search (New Zealand vs Chile). Similarly, an effort was made to recruit respondents who were not directly related to the researcher. This was done through social media posts, by asking people who did not participate in the study to recruit their contacts, and by asking respondents to recruit as well. This created a snowball effect with multiple starting points that significantly helped with the recruitment process while maintaining a certain diversity.

It is worth noticing that the results of Study 1 (see sample in Table 1) suggested that household composition (particularly, living with children) was an attribute that produced high variability in people's responses. Thus, an active effort was made to recruit more people who had children for Study 2 (see sample in ) which is why the sample in the two stages are relatively different in this regard.

As shown by Figures 5, to 9, the data collection in stages 1 and 2 continued until theoretical saturation was achieved (Bowen, 2008; Francis et al., 2010; Rowlands et al., 2015), meaning that the data gathering process continued until new interviews did not provide any new information. The amount of information was defined to be the number of codes that explained the dataset (plotted in Figures 5, to 9). The process of defining codes is explained in Section 4.7.1.

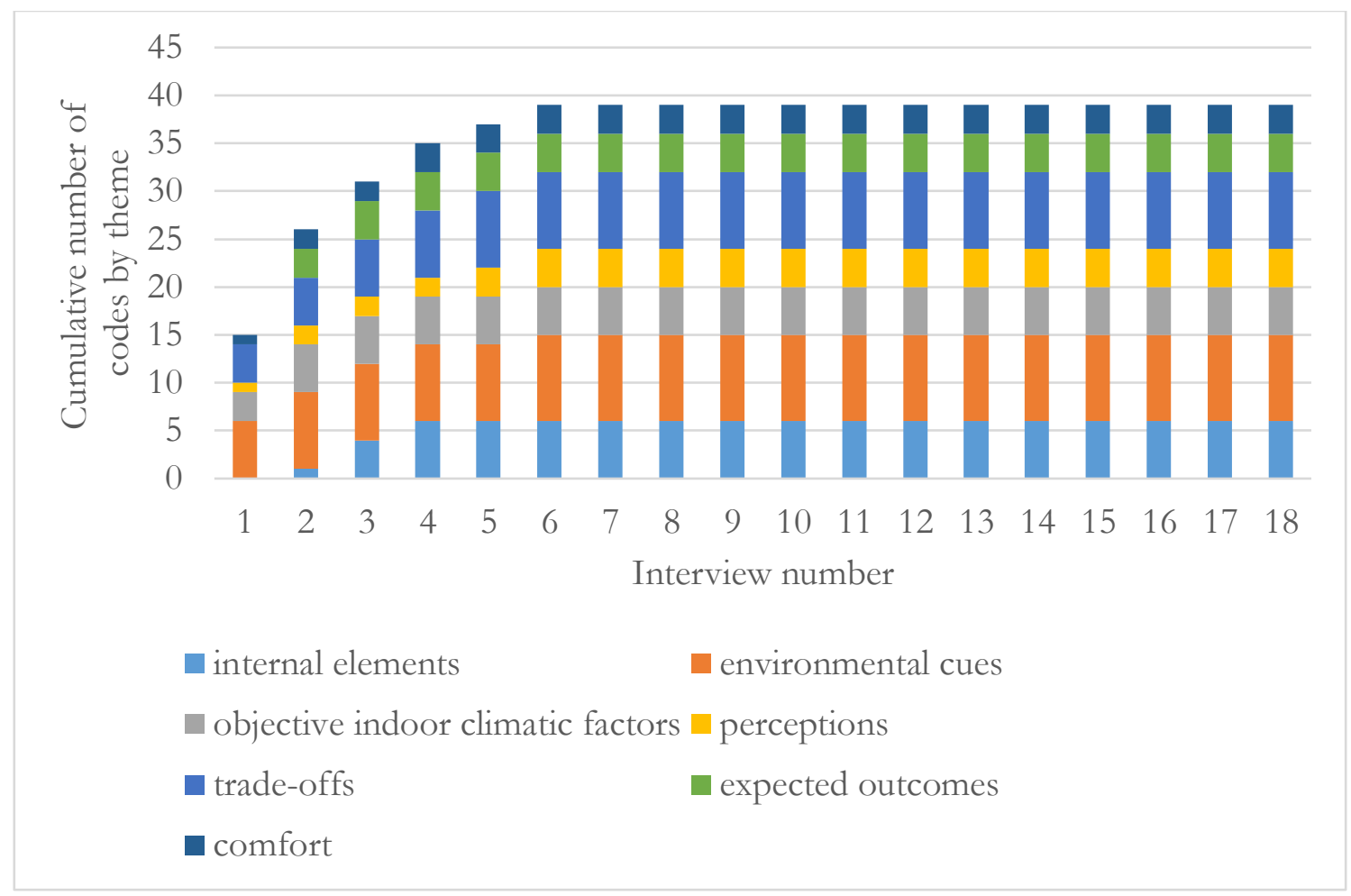


Figure 5: Cumulative number of codes related to daylight for each interview

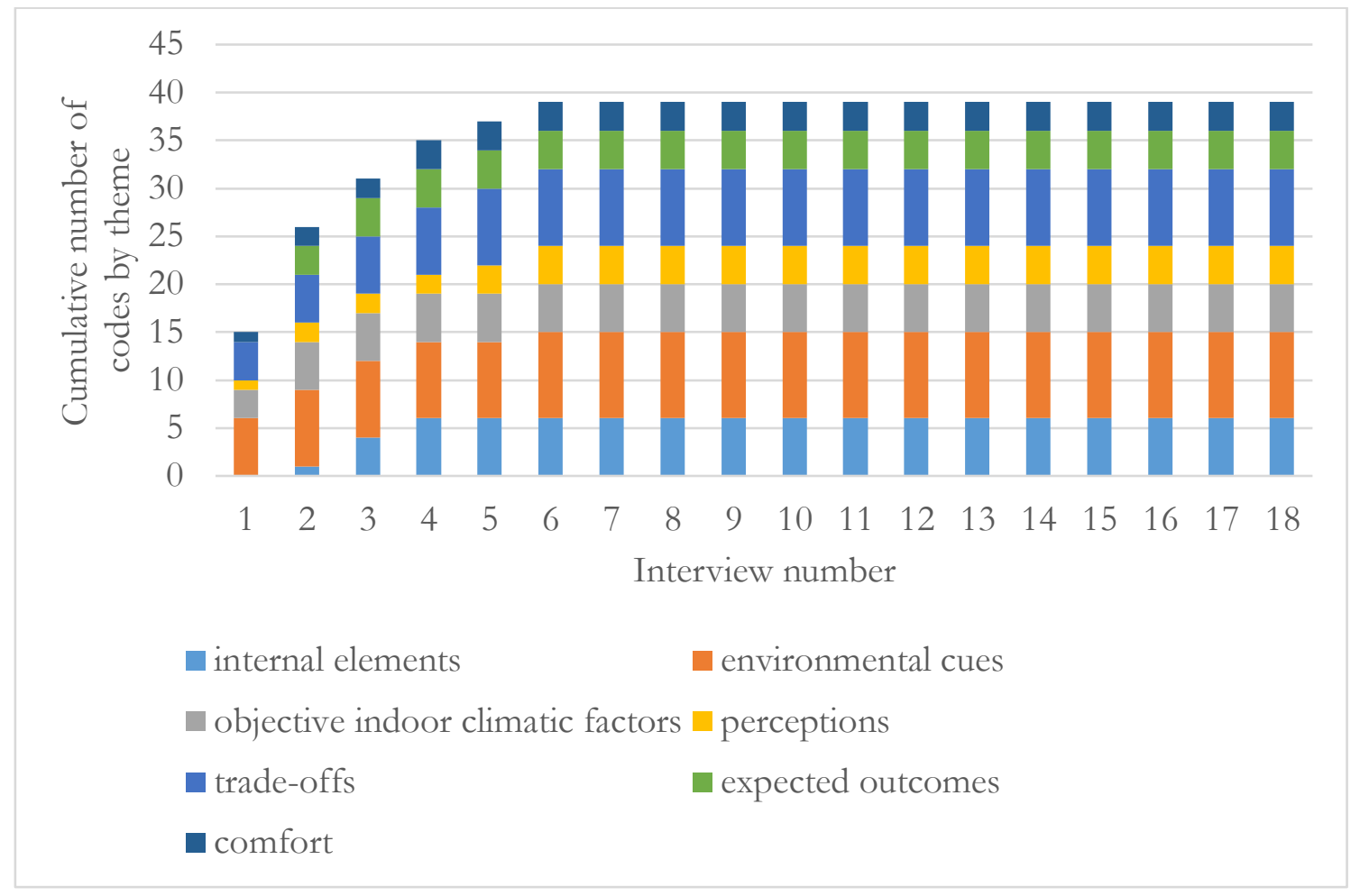

Figure 6: Cumulative number of codes related to warmness for each interview

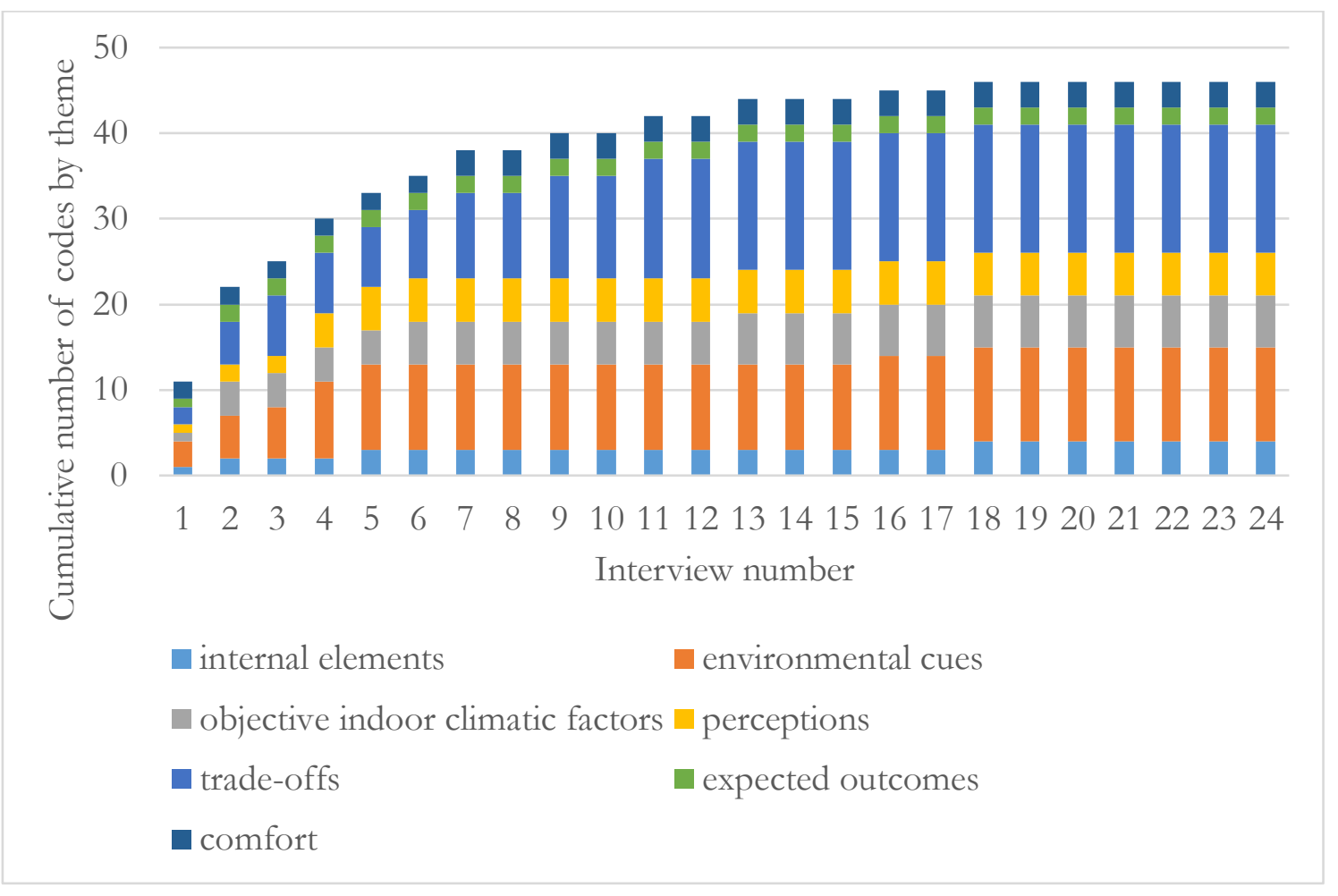

Figure 7: Cumulative number of codes related to coolness for each interview 


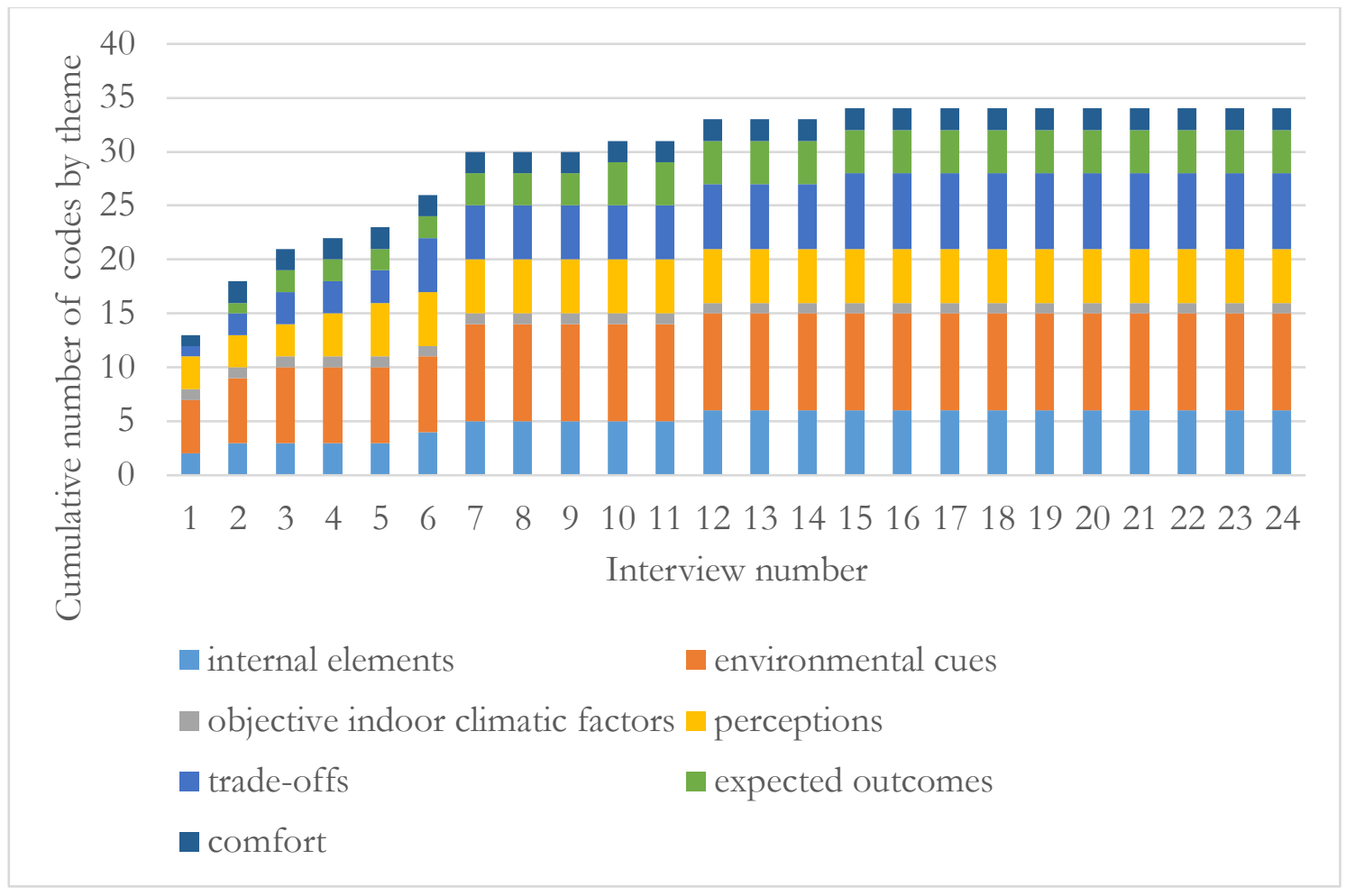

Figure 8: Cumulative number of codes related to acoustic performance for each interview

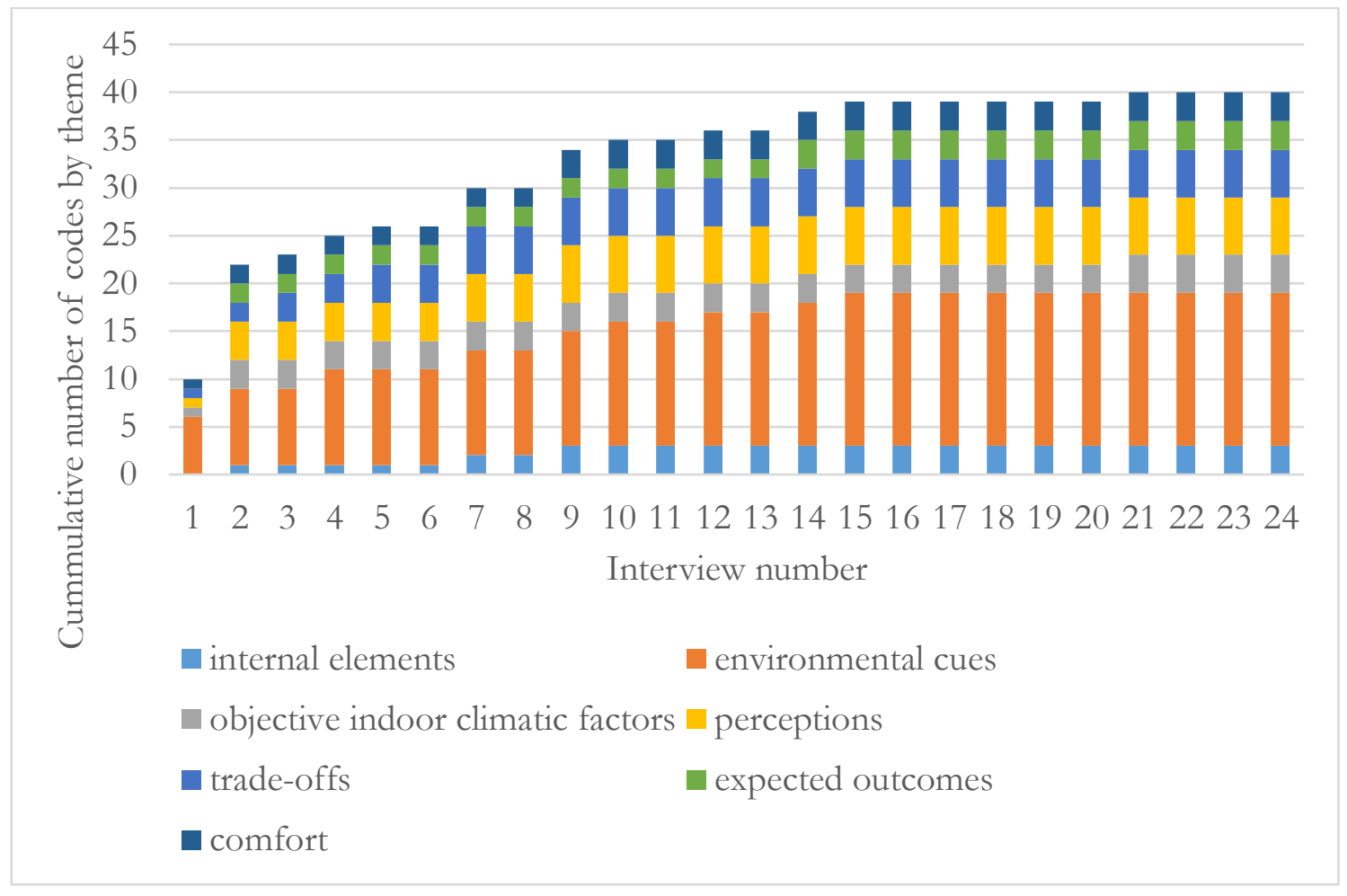

Figure 9: Cumulative number of codes related to air quality for each interview 


\subsection{Analysis of the data}

This section explains the method used to analyse the data gathered utilizing the interview described in Section 4.4. The data analysis method explained below was applied separately to each of the five domains of comfort—-warmness, coolness, air quality, acoustic, daylightwhich means that there are five datasets. However, the datasets associated with Stages 1 and 2 were analysed in slightly different ways (because the results of Study 1 were used to develop a qualitative model of comfort, while the results of Study 2 were used to verify whether new data fit in such a model). The differences are explicitly described in this section.

\subsubsection{Procedure for analysing the data}

The data - in the form of mind maps (see Figure 4)—was analysed employing a Thematic Analysis method as explained by Braun and Clarke (2006, 2012). This method proved to be a reliable system for determining patterns that explained the data and thus useful for determining the role of "the mind" in the development of comfort (i.e., research question 2). Within this research, the six steps described in Braun and Clarke (2006, 2012) were adapted as follows:

1. Familiarizing with the data: Since the data was analysed by the same person who carried out the interviews, this stage was already advanced before starting with data analysis. Proper familiarization with the data was acquired after the process of pseudo-transcription (i.e., the construction of the mind map, as shown in see Figure 4).

2. Generating initial codes: According to Braun and Clarke (2006, p. 87), this step implies "coding interesting features of the data in a systematic fashion across the entire data set". Thus, each concept or phrase-representing a node in the mind maps - was assigned one or more codes. It should be noted that, as opposed to transcribed interviews, the data generated in this research was comprised of concepts or relatively short quotes which become nodes of a mind map. Consequently, this step did not reduce the size of the data to analyse.

3. Searching for themes: After coding the data, the analysis moved up one level, and codes were sorted into themes. "A theme captures something important about the data in relation to the research question, and represents some level of patterned response or meaning within the data set" (Braun \& Clarke, 2006, p. 82). Braun and Clarke recommend using some sort of visual aid or creating a thematic map. In the case of the data for Study 1, the visual aid became the structure of the qualitative model of comfort being developed. In the case of the data gathered 
during Study 2, on the contrary, the themes were already identified (because the model had already been developed) and thus this stage was more straightforward.

4. Reviewing themes: This stage involved reviewing the candidate themes and refining them. For instance, a candidate theme might have not been supported by the data, or two candidate themes that seemed to be different might have been a single theme. Just as in the previous step of the data analysis, this was only relevant for the data associated with Study 1.

5. Defining and naming themes: This step implied identifying the essence of each theme to write a detailed analysis of it. Knowing what each theme is was as important as knowing what it was not. Again, this step was only relevant to the data gathered during Study 1.

6. Producing the report: This step is about producing a concise, coherent and logical account of the results (Braun \& Clarke, 2006). In the case of this research, the report was replaced by the model and the results explained in Chapter 5 .

It should be noted that steps 2 to 5 of the data analysis were performed iteratively. Specifically, defining the essence of a theme (step 5) often led to a review and potential redefinition of the codes (step 2) and thus steps 3 and 3 were also performed again.

\subsubsection{Considerations for analysing the data}

The analysis of the data always considered that the information provided by a single respondent was not definitive. The reason for this is that people who volunteer for this research might have mentioned codes that are not relevant to other people, and also that they could have failed to mention codes that are relevant for themselves. Nevertheless, after reaching theoretical saturation, the information acquired should be expected to be a comprehensive list of codes for each theme. For instance, there are low chances of 10 or 20 people-who do not know each other-all forgetting to report the same relevant factor affecting warmness. Thus, the list of codes for each theme very likely contains most of the codes that would be generated if everyone in the target population was interviewed (i.e., all the potentially existing codes).

It should also be noted that the compiled list of codes might contain several elements that are-in practice-irrelevant for most people. While not a definitive test, the number of respondents who mentioned a code can be used as an indicator of its relevance. For instance, since it is unlikely that a dozen respondents mention the same irrelevant attribute, a code identified in the answers of several respondents is not likely to be irrelevant. The opposite, however, is not true because it is plausible that only one in 15 or 20 interviewees mention a 
specific attribute. This means that a code that was identified only once in the data is not necessarily less important than one mentioned by all respondents.

In summary, the result of this interview is an extensive list of factors that can potentially affect—and thus be used to explain—people's feeling of Comfort. Factors mentioned repeatedly by respondents are very likely to be shared by a significant amount of people in the target population, and thus worth studying. Factors mentioned only scarcely must still be considered. Some of the factors identified through this interview might be removed from the list if further experimentation and research prove them to be irrelevant.

\subsection{Checks}

The purpose of the interview introduced in Section 4.4 is to explore how people think about comfort, identifying the factors that are relevant in the determination of comfort and how they relate to each other during the development of comfort. It becomes crucial, then, that each piece of raw data can express what the respondent wanted to say. Two measures were designed to ensure this was the case. These measures effectively transform the respondent into a second analyst who actively triangulates the raw data obtained (Patton, 1999). The first measure was to be transparent with the notes taken during the interview. That is to say, the respondents who were interviewed face to face could always see the notes being taken. Furthermore, as mentioned in Section 4.4.1, these respondents were welcome to critique and even help take notes. For obvious reasons, people who were interviewed remotely did not have that chance. The second measure was that, on top of the transparency during the interview, all respondents received a copy of the final mind map (see Figure 4)-i.e., after the pseudo transcription explained in Section 4.5 took place-for them to propose changes.

Additionally—as described in Section 4.3 (Figure 3)—the transferability (Schwandt, Lincoln, \& Guba, 2007) of the results to different contexts was assessed by performing a replication study. This means that the model was developed at one stage-based on how one group of people described comfortable homes associated with a set of indoor environmental quality domains - and then used to interpret data gathered by asking a different group of people to describe comfortable homes associated with a different set of indoor environmental quality domains. Moreover, the chances that the results of this research are applicable in other contexts are increased by interviewing people from two different countries: Chile and New Zealand. This makes it less likely that the results are highly dependable on socio-cultural factors.

Finally, in order to ensure that the results-i.e., the processed data, with codes and 
themes assigned-were transparently available, all the data of this research was uploaded to an interactive website that allows people to browse and explore the model developed during this research. These results are available at Buildingsforpeople.org.

\subsection{Ethics}

This project was reviewed and approved by the Victoria University of Wellington Human Ethics Committee (ID 0000027329). 


\section{A QUALITATIVE FEELING OF COMFORT MODEL}

"When you are lost in information, an information map is kind of useful"

DAVID MCCANDLESS, TED 2010

Chapters 2 and 3 of this thesis argued that even if building science often introduces the concept of comfort as a subjective state of the mind (e.g., Auliciems, 1981, 1983; de Dear et al., 2016), its models of comfort tend to depend almost exclusively on objective physical factors (e.g., Fountain \& Huizenga, 1996; Jakubiec \& Reinhart, 2012; Sarey Khanie, Stoll, Einhäuser, Wienold, \& Andersen, 2017; van Hoof, Mazej, \& Hensen, 2010; Wienold \& Christoffersen, 2006; Wienold et al., 2019). The reason for this seems to be pragmatism, meaning that building scientists have disregarded "the mind" motivated by the fear of the complexity it might introduce to their research and practice (e.g., Reinhart, 2014, p. 27). This chapter introduces a new qualitative model of comfort-the model of the Feeling of Comfort - which has the advantage of embracing the fact that comfort is a state of the mind while being simple enough to serve as a framework for approaching comfort research and practice, and as a map for understanding how the Feeling of Comfort develops. This model was developed from the data gathered through the interviews performed in Study 1, in which respondents described a "warm dwelling" and a "dwelling with good natural light".

It is worth mentioning that the data presented in what remains of this chapter correspond to phrases and statements contained in the respondents' descriptions of warm dwellings and dwellings with good natural light. Because showing all the data would make this chapter exceedingly long and painful to read, only selected quotes are shown in the text. These quotes were chosen based on their representativeness of the argument being given, and because they were expressed by respondents of different countries, gender, household compositions, or other attributes. A list with all the codes identified in all the responses is available in Appendix IV. A copy of all the qualitative data can be made available by request to the author. 


\subsection{The Feeling of Comfort}

Building scientists mostly express comfort as the absence of physiological effort. For instance, thermal comfort is commonly reported through scales that go from "cold" to "hot" and have a single "comfortable" or "neutral" rate that represents the optimal condition (Auliciems \& Szokolay, 1997; Gagge et al., 1967; Rohles, 2007). This is supported by the fact that "under thermally near neutral laboratory conditions, minimally experienced temperature sensation, discomfort, and, an even more integrated response of 'unpleasantness'... are coincident" (Auliciems, 1981, p. 113). Similarly, visual comfort is mainly understood as the absence of uncomfortable lighting conditions (Jakubiec \& Reinhart, 2012; Sawicki \& Wolska, 2015; Wienold et al., 2019). Buildings must, therefore, provide enough daylight to be able to see while avoiding glare and overheating of the space (Mardaljevic, 1995; Nabil \& Mardaljevic, 2006). Thus, the de facto definition of comfort has mostly focused on what is not uncomfortable, as opposed to what is comfortable.

While the focus on what is "not uncomfortable" can potentially make sense for officeswhere the environmental conditions often prevent people from performing their duties-it does not seem to be appropriate for residential settings. On the contrary, respondents' descriptions of "a warm dwelling" and "a dwelling with good natural light" suggested that comfort is a goal in itself and not a means for being productive:

"I guess it is about quality of life" ... "II] cannot put my finger in anything different..." ... "[A cold house] is just uncomfortable"

Respondent 13 - Study 1, New Zealand

"There is nothing better than a warm floor"... "You want to be in a warm house"

Respondent 6 - Study 1, Chile

"[The difference between a cold and a warm apartment is] mainly space, comfort, luminosity and tranquillity"

Respondent 9 - Study 1, Chile

"I personally really like daylight"

Respondent 12 - Study 1, New Zealand

"For me, one of the most important things in buying a house was having a place that is bright because otherwise you go inside and it just feels depressing"

Respondent 14 - Study 1, New Zealand 
Moreover, respondents' descriptions reflected how comfortable homes are not just "not uncomfortable" or "neutral" but can reach the point at which they become a place where people actively want to go to. This positive connotation of comfort is sometimes called “delight” (Heschong, 1979; Steemers \& Steane, 2004, p. 6) and was expressed by respondents who mentioned that comfort can reach the realm of happiness, quality of life, friendship and belonging. Thus, even if, in principle, people's physiological system can only go from neutral (i.e., no physiological efforts) to unpleasant (i.e., high physiological effort) ${ }^{5}$, "the mind" seems to be able to interpret comfort as a positive construct. This further emphasizes the need for incorporating "the mind" in comfort research, as focusing almost exclusively on physiological elements implies omitting the potentially positive effects of the built environment over people's wellbeing.

"In the warm house, in winter more than in other seasons, it gives you a surplus. A wellbeing surplus that you appreciate" ... "You actually want to get [to a warm house]"

Respondent 4 - Study 1, Chile

"There is nothing nicer than using the natural light... I love it"

Respondent 6 - Study 1, Chile

"In terms of guests, you know, you feel that people are comfortable [in a warm house ]"... "I I associate light with] Life and energy and activity"

Respondent 11 - Study 1, New Zealand

"We are happier now in a warmer house"

Respondent 10 - Study 1, New Zealand

It was also possible to infer, from respondents' answers, that their assessment of the indoor environment was often based on automatic, overwhelming, and full-body experiences that happen to them and that are often hard to explain in words. This was more evident in the case of discomfort than comfort because respondents described the former as an absolute full-body negative experience and the latter as a milder-yet positive and hardly explainable-experience. The fact that discomfort has a stronger effect on people's dissatisfaction than comfort on people's satisfaction is supported by the results from Kin and de Dear (2012). Some of the respondents expressed this as follows:

\footnotetext{
5 The phenomenon of Alliesthesia-i.e., when sensations produce pleasant feelings-will be discussed in section 5.2.3, when describing the role of Perceptions
} 
"When it is cold I cannot think... I cannot focus" ... "I am annoyed... restless"

Respondent 8 - Study 1, Chile

"If it is comfortable, or if it is warm... then I just do not focus on the temperature"... "I focus more on the temperature if it is cold"

Respondent 12 - Study 1, New Zealand

"Heat [destroys] me, it [ruins] my head"

Respondent 2 - Study 1, Chile

"For me, a part of being warm is feeling warm, and feeling warm is like a perception"

Respondent 14 - Study 1, New Zealand

"[You need to turn the lights on] when you can't see well, I mean, you need to turn on the lights when it bothers you, like... I do not know how to explain"

Respondent 15 - Study 1, New Zealand

"Daylight helps... not for the decoration, but for the vision of... I don't know"

Respondent 6 - Study 1, Chile

"I think [having good daylight] is not so much what you can do, it is how you feel. It is about the feeling" ... "[Because for functioning] you can always switch the lights on"

Respondent 18 - Study 1, New Zealand

The traits reflected in the quotes above-where comfort/discomfort is seen as an automatic, overwhelming and full-body experience-are usually associated with emotions (Barrett, Ochsner, \& Gross, 2007; Mauss, McCarter, Levenson, Wilhelm, \& Gross, 2005). This insight inspired the label Feeling of Comfort.

\subsection{How people's Feeling of Comfort develops}

This section formally introduces a model of the Feeling of Comfort and explains its different components. This model attempts to explain the process through which the Feeling of Comfort develops. As in other models (e.g., Aries et al., 2010, p. 534; Auliciems, 1981; Luo, 2020, p. 132), the model introduced in this chapter posits that the development of the Feeling of Comfort starts from the relationship between the built environment and the individual, goes through a perception layer and ends in the Feeling of Comfort itself. However, this model differs from traditional models in two ways. First, in that not only Perceptions influence comfort, but also Expected Outcomes and Trade-Offs; and second, in that perceptions are understood to be the result of cognitive processes and are not directly 
associated with sensations (e.g., perceptions are not necessarily limited to thermal sensation and glare).

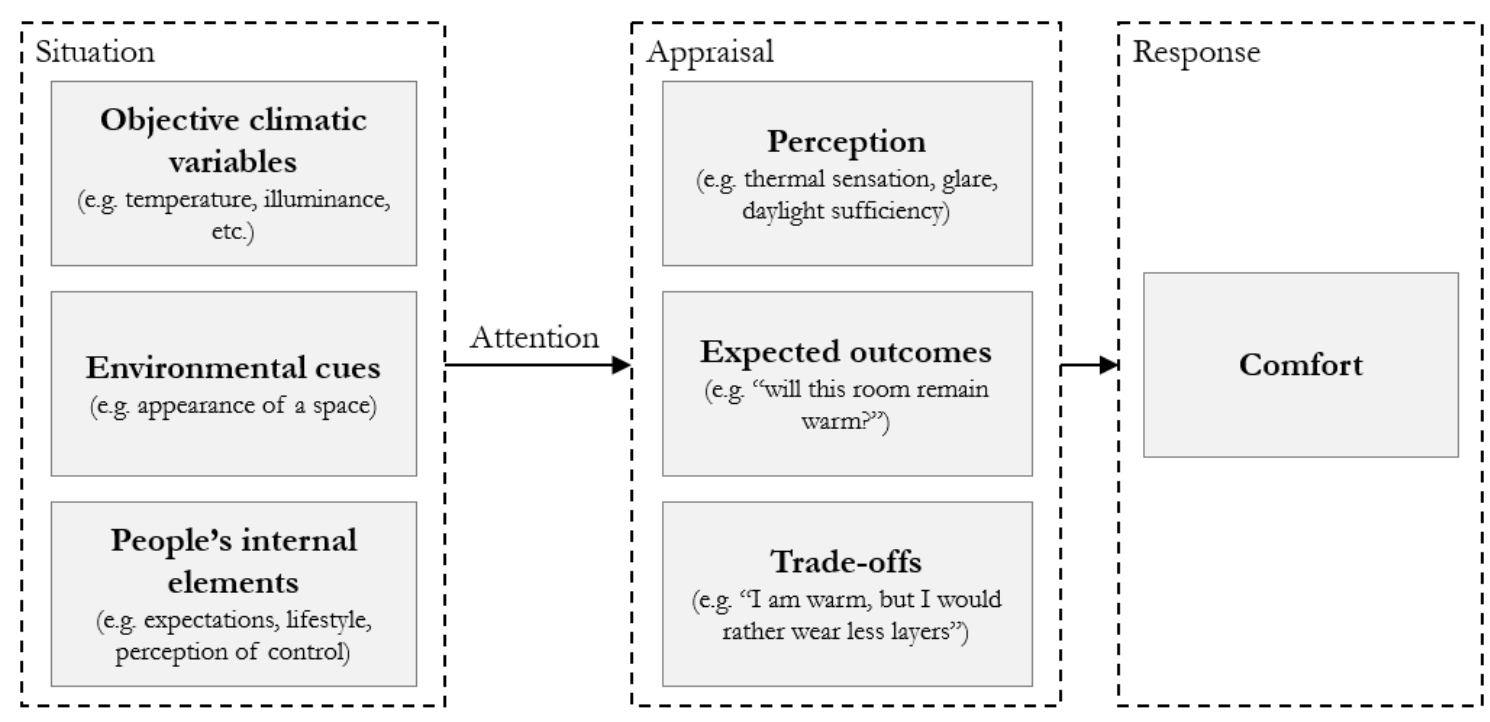

Figure 10: The qualitative model of the Feeling of Comfort

What remains of this chapter explains each of the elements shown in Figure 10 (e.g., Perception, Trade-offs, etc.), showing examples of sub-elements within each of these blocks and presenting some of the data that allowed identifying each element. It is worth mentioning that these sub-elements are not presumed to be universal. On the contrary, they are expected to change from culture to culture and from climate to climate. For instance, respondents from Chile — particularly from the, during summer, hot and sunny Santiago—seemed to be more aware of the nuisances that excessive daylight can cause than those from New Zealand.

"I do not like being exposed to the sun. I like daylight to get in, but not to be myself under the sun. I mean, not being there burning, getting a suntan inside the house"

Respondent 1 - Study 1, Chile

"I do not find the sun pleasant, generally. The truth is, I do not like to be exposed to the direct sun. Not in summer"... "I love big windows, but hopefully, the sun will not get inside"

Respondent 2 - Study 1, Chile

"I get there in the evening, and I can read a book or drink a beer, and the sun is over me"

Respondent 15 - Study 1, New Zealand

Despite these differences, the descriptions given by respondents from both countries contained the main elements of the model. That is to say, Respondents from both countries mentioned Objective climatic variables, Environmental Cues, Internal Elements, 
Perceptions, Expected outcomes and Trade-offs. Consequently, this chapter argues that the main elements of the model are likely to be universal, and thus they should always be considered when performing comfort research. The list of sub-elements highlighted in each of these sections, on the contrary, is simply an extensive collection of exemplars that helps to explain the model through more concrete experiences and words. Also, since it was compiled from data from two different countries, this list of exemplars can be used as the basis for new research and practice. In other words, researchers and designers that want to use the model of the Feeling of Comfort select the sub-elements that seem appropriate for their context, exclude those deemed irrelevant and create new ones that seem to be needed.

\subsubsection{The situation}

The situation is the starting point for the development of the Feeling of Comfort. It includes not only physical factors (e.g., temperature, humidity, size of the windows, and colour of walls) but also peoples internal (i.e., non-physical) elements (e.g., lifestyle, knowledge and experience about similar situations). In other words, the situation includes all the psychologically relevant factors and not simply the physical surroundings, thus holding the same connotation as in some studies of emotions (Gross, 2013).

This model further divides the physical world into what de Dear et al. (2016) call objective indoor climatic variables (e.g., temperature, illuminance, humidity) and other nonquantifiable and even non-directly-related environmental cues (e.g., the appearance of a room or the views outside). The distinction between these two groups intends to respect traditional building science's models of comfort, which mainly focus on "objective indoor climatic variables".

\section{Objective climatic variables}

Objective indoor climatic variables are those physical aspects of the environment that building scientists have traditionally measured and utilized for comfort models. These factors are a consequence of the interaction between a dwelling's design (e.g., geometry and materials), its surroundings, placement (e.g., orientation), and the climate of its location. The main characteristic of these variables is that they are quantifiable in physical terms and human physiological systems can often sense them. Thus, objective climatic factors can affect people's comfort through the path of sensations and perceptions; that is to say, through physical/physiological mechanisms.

Table 3 shows the Objective indoor climatic variables identified as relevant for warmness and daylight. As stated in Section 4.7, the variables in Table 3 were identified through the 
Thematic Analysis. In the language of such a method, these variables are codes; in other words, relevant ideas that one or more respondents mentioned during the interviews.

Table 3: Objective indoor climatic variables identified for daylight and warmness

\begin{tabular}{|c|c|}
\hline Warmness & Daylight \\
\hline - Air temperature & - Direction of light \\
\hline - Exposure to solar radiation & - Direct vs diffuse light \\
\hline - Floor contact temperature & \\
\hline - $\quad$ Air leakage & \\
\hline - Bed temperature & \\
\hline
\end{tabular}

Note: Descriptions of all these elements are available in Appendix IV

Respondents mentioned Objective indoor climatic variables in different ways when describing comfortable homes. For example, when describing a dwelling with good natural light, people reported that the proportion of direct and diffuse daylight was a relevant variable in their assessment of the daylight quality. For instance, while daylight is considered a good thing, the strength of direct sunlight can be seen as detrimental to people's overall experience. As some participants mentioned:

"I love big windows, but hopefully, the sun will not get inside"

Respondent 2 - Study 1, Chile

"[Direct sun] is good, but light is much more important to me"

Respondent 16 - Study 1, New Zealand

"It does not have to be straight sun, or powerful sun all the time... Because I am thinking it is going to get hotter and hotter in the world"

Respondent 18 - Study 1, New Zealand

While the floor contact temperature has been mentioned in the literature (e.g., ASHRAE, 2010), this variable has not often received significant attention. Respondents, however, explained how the floor contact temperature is a relevant factor in their assessment of warmness:

"[We] sacrificed warmness because it looked good. But if we had preferred warmness, we would have put carpet over the floor... a wooden floor normally feels cold"

Respondent 10 - Study 1, New Zealand 
"You can walk around barefoot. Or, ok, with slippers or something like that, but not with ski boots inside the house. Or with thermal socks"

Respondent 3 - Study 1, Chile

The results of this study show that the Objective climatic factors most often utilized by building science's literature are, indeed, those that the respondents of this research consider relevant. Nevertheless, the results also revealed two factors that have apparently not been considered: the direction of daylight, and the temperature of the bed. Specifically, people seem to notice when the light comes from above and assess it differently (not necessarily better) to lateral light. Likewise, going to sleep in a cold bed seems to be a significantly unpleasant situation, significantly affecting the overall experience in a home.

\section{"[I want daylight everywhere,] even if the light comes from the ceiling"}

Respondent 1 - Study 1, Chile

"Light from above"

Respondent 6 - Study 1, Chile

"The fact that the sheets are cold is something that took some time for me to get used to... in my old house, I mean, I was scared of going to bed"

Respondent 10 - Study 1, New Zealand

\section{Environmental cues}

Environmental cues are those elements of the environment that-even if hard or impossible to quantify, or apparently unrelated to the phenomenon being studied-are used by people both consciously and unconsciously to judge whether a dwelling is comfortable or not. Contrary to objective climatic factors-which can influence comfort through physical/physiological mechanisms-environmental cues contribute to the determination of comfort through cognition. An example of this happens when the physical factors of one domain affect the perceptions of another domain. For instance, people's thermal sensation and visual comfort and perceptions are known to be interrelated (Chinazzo et al., 2019; Chinazzo, Wienold, \& Andersen, 2020; Chinazzo, Wienold, et al., 2018a; Golasi et al., 2019).

Table 4 shows the Environmental cues identified as relevant for the domains of daylight and warmness. 


\begin{tabular}{lll}
\hline \multicolumn{1}{c}{ Warmness } & \multicolumn{1}{c}{ Daylight } \\
\hline - Site characteristics & $\bullet \quad$ Site characteristics \\
- Size of the space & $\bullet \quad$ Size of the space \\
- Orientation & $\bullet \quad$ Orientation \\
- Condensation and mould & $\bullet \quad$ Colour of the space \\
- Thermal insulation & $\bullet \quad$ Availability of windows and skylights \\
- Heating and cooling system & \\
- Affective attributes & \\
- Materials that feel warm & & \\
\hline & Protection from moisture & \\
\hline
\end{tabular}

Note: Descriptions of all these elements are available in Appendix IV

Apart from some cross-domain interactions mentioned earlier, environmental cues can influence people's comfort by informing the inferences they make about what will happen in the future (see Section 5.2.3 for the relevance of Expected outcomes). For instance, the fact that a dwelling has a heating system seems to help people feel more comfortable because they know that, if it gets cold, they will be able to keep the dwelling warm.

"[A warm dwelling] has a heat pump... There is no other option in Wellington”

Respondent 10 - Study 1, New Zealand

"[In a warm home] there is also heating in each room... In each room I have installed [what] I think you would call it a panel heater"

Respondent 11 - Study 1, New Zealand

"[If a house is warm it] implies as well that it has some way of heating itself up"

Respondent 14 - Study 1, New Zealand

Environmental Cues seem to be relevant for people when making long-term inferences as well. For instance, when searching for a new home, people often take note of the orientation and site characteristics to judge and compare each dwelling with others. While these inferences are very long-term, their existence still reflects how people are constantly aware of the future when assessing the quality and comfort of a space. This is discussed in more detail in Section 5.2.3 when explaining the role of Expected outcomes. Participants 
expressed this as follows:

"The most important thing is the orientation of the house... if it is facing South, then it is going to be impossible to have a lot of daylight"

Respondent 14 - Study 1, New Zealand

"More windows lead to more [not necessarily better] light [as] it depends on the direction the window is facing"

Respondent 2 - Study 1, Chile

"Unobstructed windows... I mean, that nothing is stopping the light from coming into the house"... "Not too much vegetation in the surroundings"... "Not having a retaining wall close by, ideally"

Respondent 10 - Study 1, New Zealand

The code of affective attributes gathers environmental cues that describe how welcoming and liveable a home is. While this would require some research, these elements likely arise from the fact that the word "warm" is given this meaning in Latin American Spanish (i.e., cálido or cálida). However, despite the plausibility explanation, a respondent in New Zealand also used affective attributes to describe a warm home.

"It is impossible not to relate it with the feeling of the liveability of the place... I will not necessarily evaluate the issue of temperature"... "It can involve something more, like belonging"

Respondent 6 - Study 1, Chile

"I picture it with big, with high ceilings"... "It is much more beautiful with high ceilings and a warm house does not need to be small, necessarily"

Respondent 3 - Study 1, Chile

"I do like to feel that it is, hmmm, that there is an outlook. So when I look out of the window, there is something to see"... "I want some sense of space"... "I quite like having neighbours, even though [when] moving I did not know who they would be. I did not know what kind of people they would be. But I wanted to have some human contact"

Respondent 18 - Study 1, New Zealand

This kind of linguistic and cultural difference stresses the need for scrutinizing the questionnaire-driven approach to research. For instance, it is known that people's responses to thermal sensation scales vary depending on the type of scale being used and that the different verbal anchors available for describing thermal sensation (e.g., cool, cold, slightly warm) are not equidistant (Schweiker et al., 2017). Moreover, it is also known that different 
people perceive scales differently and that their responses vary with contextual factors such as climate and language (Schweiker, André, et al., 2020).

\section{People's internal elements}

Comfort is a subjective state of the mind and therefore its starting point should be the individual. Nevertheless, as discussed in Section 2.3, comfort studies and theories have mostly ignored the non-physical attributes of people. These non-physical personal attributes are what the Feeling of Comfort model calls "Internal elements" which, together with the cognitive processes explained in Section 5.2.2, comprise what the theoretical framework in Figure 2 calls "the mind".

Internal elements are personal attributes that shape the meaning people give meaning to specific situations and thus they are crucial for determining people's Feeling of Comfort. These elements are the ones that introduce subjectivity to the model presented in this chapter. Table 5 lists the internal elements identified as relevant for the domains of warmness and daylight.

Table 5: Internal elements identified for daylight and warmness

\begin{tabular}{lll}
\hline \multicolumn{1}{c}{ Warmness } & \multicolumn{1}{c}{ Daylight } \\
\hline - & \multicolumn{1}{c}{ Times of occupancy } & \\
- & Dwelling typology occupancy \\
- & Household composition \\
- & Lifestyle \\
- Thermal preference & \\
- Budget
\end{tabular}

Note: Descriptions of all these elements are available in Appendix IV

One example of an Internal Element is people's lifestyle. For instance, some respondents mentioned that they like to socialize with families (including children) while others do not, and some people are used to wearing more clothing than others.

"I do not wear a $t$-shirt in the winter. But again, that is cultural. We are used to wearing a pullover in the winter. But, yeah no, but I mean, I come home, I turn on the heat pump and then I will go on doing what I am doing and it is fine”

Respondent 11 - Study 1, New Zealand

"Where children can play... not a museum-like apartment" 
Another example is the typology of the dwelling people are looking for. This element only applies when people are searching for a new home, meaning that it is used by people to make long term inferences to choose a comfortable property. For example, one respondent said the following:

"When I searched for my apartment, Yes, [orientation] was crucial. In houses, in general, since houses are detached, you have some freedom to find daylight or windows where you want to"

Respondent 2 - Study 1, Chile

Also, people seem to have a predefined budget for utility bills which can significantly affect people's behaviour and experience in their homes. For example, some respondents mentioned that they were simply not willing to pay all the money necessary to maintain their homes at a pleasant temperature. Others, on the contrary, are willing to pay as much as they need to.

"It would be ideal not to turn [the heater] on because it is expensive, but I think it is worth it"

Respondent 4 - Study 1, Chile

"Well, I guess in features, [in a warm home] there is a form of heating which is economical... because if it is not economical I am not going to use it"

Respondent 18 - Study 1, New Zealand

"[Heating in] my building is very expensive, outrageous. In the one before I used to use it. But here, in the end, since I live alone, I do not share expenses anymore... But still, it is important to me... I could not live without a heater... today I bought a heater"

Respondent 6 - Study 1, Chile

It might be important to notice that the budget attribute was identified in this research even though it targeted people who are not within demographics usually associated with energy-poor people (i.e., it targeted people who can afford to purchase a dwelling). Thus, people's Budget seems to be more associated with "mental accounting" (Thaler, 1999); i.e., with the fact that people group expenditures into categories (i.e., accounts) and control the budget of each of them separately.

It can be seen in Table 5 that the internal elements identified are not very many. On the contrary, it is a list of a very manageable size. This is relevant because it implies that embracing some of the subjectivity that is intrinsic to comfort will not necessarily result in overly complex models. For example, the elements listed in Table 5 can be used for 
performing more focusing comfort studies (e.g., addressing "Times of occupancy" by targeting only people who work from home, or "household composition" by recruiting people who live by themselves).

\subsubsection{The attention}

As explained in Section 2.2.2, Perceptions are how people's minds interpret sensations, thus they are the result of cognitive processes. A requirement for these cognitive processes to happen is that "the mind" needs to be aware of the stimuli it is supposed to interpret. In other words, the situation — as described in Section 5.2.1-needs to capture "the mind's" attention.

It is known that some external stimuli are not strong enough to produce perceptions (Feher, 2012; Schiffman, 2000, p. 24). This might partly explain why respondents' suggested that they are not always attending to the situations they are involved in. On the contrary, it would seem that situations whose environmental conditions offer a relatively neutral set of perceptions can sometimes go unnoticed while adverse ones are more likely to be noticed.

"If it is comfortable, or if it is warm... then I just do not focus on the temperature"

Respondent 12 - Study 1, New Zealand

"When it is cold I cannot think... I cannot focus"... "I am annoyed, I am restless"

Respondent 8 - Study 1, Chile

"You need to turn on lights when it bothers you, like... I do not know how to explain"

Respondent 15 - Study 1, New Zealand

But not only unpleasant situations can capture people's attention. On the contrary, it would seem that relatively abrupt changes in the environmental conditions of the situation a person is involved in-e.g., when moving from one space to another-can force people to attend to their new situation. This can happen not only when the environmental conditions become more adverse but also in the opposite direction. Respondents expressed this as follows: 
"The feeling of arrival to the house is crucial"... "You open the door and feel the temperature change and say Aaaah, nice, how pleasant"

Respondent 4 - Study 1, Chile

"[A place is too dark when...] it is like the feeling you get when you go in. That, you step out of the bright sunshine outside, and you step into a place... which feels significantly darker"

Respondent 14 - Study 1, New Zealand

So far, everything that this section has mentioned about attention can be relatively easily associated with physiology and thus it supports building science's current practices. Specifically, the fact that unpleasant situations are more likely to evoke attention is consistent with the widespread idea that comfort can be equated to physiological neutrality or lack of nuisances (e.g., ASHRAE, 1999, 2010; Jakubiec \& Reinhart, 2015). Likewise, these abrupt positive changes in a person's situation with alliesthesia (Cabanac, 1971; de Dear, 2011; Parkinson \& de Dear, 2015); that is to say, the fact that, if a certain variable in a person's body has been displaced from its set-point, then returning it to its natural state through external stimuli will generate the feeling of pleasure (de Dear, 2011). However, it is important to remember that the situation includes a strong psychological component and thus that not only physical/physiological factors can force people to attend a situation. This is consistent, for instance, with the literature on emotions (Gross, 1998, 2015) and with the fact that respondents mentioned how a situation can capture their attention by making them worry about other people.

"[A warm home] allows me not to be [scared] of the children getting uncovered during the night"

Respondent 1 - Study 1, Chile

Additionally, it is well known that indoor environmental quality can influence people's productivity (e.g., Leaman \& Bordass, 1999; Lowry, 2018; Mujan et al., 2019), a functional connotation of comfort can be easily found in building science's comfort literature. Respondents seem to agree with this, as some of them mentioned that some situations, even if not particularly unpleasant, are likely to capture their attention if they make it difficult to achieve their goals.

"Your own shadow darkens your view... You are opening a yoghurt [in my kitchen] and your own shadow bothers you" ... "With natural light that usually does not happen"

Respondent 4 - Study 1, Chile 
"If I am already wearing glasses, if I am trying to read and I cannot really see because the light is not good, then that is just an added kind of nuisance"

Respondent 11 - Study 1, New Zealand

In summary, it would seem that people are less likely to attend situations in which the indoor environmental conditions evoke a neutral set of perceptions (e.g., lack of nuisances) than others. This has a physiological basis, as not all external stimuli are strong enough to evoke Perceptions (Feher, 2012; Schiffman, 2000, p. 24), and thus neither can they start the development of the Feeling of Comfort. However, relatively abrupt changes in the environmental conditions of the situation seem to be able to force attention, even if the change ultimately offers a more pleasant situation. Finally, but probably not less important, it is necessary to acknowledge that not all arguably similar environmental conditions (or changes in them) are equally likely to capture a person's attention. This might happen, for instance, because people's goals differ (e.g., reading a book vs enjoying a beer) and thus these situations also differ; and second, because people might pay attention to a situation due to psychological reasons. E.g., hearing an elderly cough or seeing a child wearing no shoes might force attention to the temperature in the room.

\subsubsection{The appraisal}

After a situation captures a person's attention, it will result in the feeling of a certain degree of comfort. Being a state of the mind, comfort is seen as the result of cognitive processes or appraisals. These appraisals will be based on the elements in the Situation and then produce the Feeling of Comfort.

Probably the biggest difference between traditional building science's comfort models and the model presented in this chapter lies in the fact that, while the former tend to rely only on Perceptions, the latter adds two new appraisals people make when developing their Feeling of Comfort. The first one-Expected outcomes-represents the future and reflects the fact that people are always thinking ahead of the present. The second one-assessing the Trade-offs they have made-represents the influence of past actions on the present, and reflects the fact that people always put comfort in context and are aware that improving one aspect of their lives can affect others. These two additions are complementary to people's Perceptions, which represent the here and now and reflect the fact that people can sense their environment.

\section{Perceptions}

Perceptions are the meaning people give to sensations (de Dear, 2011, p. 110). In other 
words, sensations are the first contact between environment and body, while perceptions require cognition (Coren et al., 2004). For instance, being the result of a cognitive process that gives meaning to the sensory data, the description of a room as being "too hot" or "too dark" should formally be called perception. Nevertheless, thermal comfort studies often use the concept of thermal sensation (Djongyang et al., 2010; Schweiker et al., 2017). This is not necessarily surprising because these concepts are often treated as synonyms (Coren et al., 2004, p. 8).

Table 6: Perceptions identified for daylight and warmness

\begin{tabular}{lll}
\hline \multicolumn{1}{c}{ Warmness } & \multicolumn{1}{c}{ Daylight } \\
\hline - Daylight sufficiency & $\bullet$ & Daylight sufficiency \\
- Sensation when entering a space & $\bullet$ & Sensation when entering a space \\
- Thermal sensation & $\bullet$ & Feeling of confinement \\
& $\bullet$ & Cleanliness of the space \\
& $\bullet$ & Sense of connection with the exterior \\
& $\bullet$ & Attractiveness of the space \\
& $\bullet$ & Glare \\
\hline
\end{tabular}

Note: Descriptions of all these elements are available in Appendix IV

People's Perceptions indicate how acceptable is the environment, and thus they represent the here and now. Recognizing this, building science's comfort research has acknowledged perceptions for a long time. However, it has mostly focused on those that can be directly associated with sensing organs such as thermal sensation (in people's skin), glare (in people's eyes), loudness (in people's ears). The model presented in this chapter recognizes all these perceptions and posits the existence of a second group that is much more related to cognitive processes. For instance, the Feeling of Confinement-mentioned by people when describing a dwelling with good daylight—cannot be easily associated with people's physiological system. Table 6 shows the perceptions identified as relevant for daylight and warmness.

Perceptions are affected by all the elements of the situation. Of course, as widely studied, objective climatic variables affect people's thermal and visual comfort through sensations. In other words, objective climatic variables can affect perceptions through physical and physiological phenomena. For instance, drafts affect people's thermal sensation, and the amount of light affects the perception of daylight sufficiency, as described by some participants: 
"It has no drafts. Drafts are unbearable. You can be warm and when drafts come in [it is horrible]"

Respondent 3 - Study 1, Chile

"Probably the ideal amount of light would mean that I can, like, read my book without turning the lights on. Not a Kindle, an actual book"

Respondent 16 - Study 1, New Zealand

In contrast, the mechanism through which environmental cues affect perceptions may be understood as the effect of intuitive judgments (Evans, 2003, 2008; Kahneman, 2003; Stanovich \& West, 2000; Thaler \& Sunstein, 2008). For instance, a room that looks like a refrigerator may feel cold only because people's brains are trained to associate refrigerators with cold (Rohles, 2007). Similarly, the colour and amount of the light in a room changes people's thermal sensation (Chinazzo et al., 2019; Chinazzo, Wienold, et al., 2018a), although Chinazzo (personal communication, 27 of February 2019) warns that her results are valid for extreme colour stimuli and thus might not be applicable for normal architectural contexts. Despite this warning, respondents expressed the importance of elements that are not related to warmness with their thermal sensation.

"Feeling like there is a separation [between inside and outside environments], makes it feel warmer"

Respondent 12 - Study 1, New Zealand

"It is important for me that, for a house to feel warm, that it has got a lot of light... I think that a house feels warmer if it is bright. It is a perception"

Respondent 14 - Study 1, New Zealand

The effect of Internal elements over perceptions changes the way the situation is assessed. For instance, living with children or clothing preferences can greatly determine when a room is considered to be "too cold" or "too hot".

"The thing is that, when you do not have children, cold does not matter... I do not care about cold if I am not with my kids"

Respondent 2 - Study 1, Chile

"I do not wear a t-shirt in the winter. But again, that is cultural. We are used to wearing a pullover in the winter... I come home, I turn on the heat pump and then I will go on doing what I am doing and it is fine"

Respondent 11 - Study 1, New Zealand

On the other hand, the mechanism through which perceptions affect comfort has been the main focus of building science's comfort research. In fact, comfort is often assumed to 
exist when people's physiological responses are minimized (i.e., physiological neutrality). Some respondents specifically mentioned that perceptions are, indeed, relevant and that they can be detrimental to their Feeling of Comfort.

For instance, respondents mentioned the fact that thermal sensation-expressed in terms such as "not too cold and not too hot", "pleasant temperature" or "nice temperature"-is relevant in the determination of warmness. Similarly, people often mentioned that having enough contrast and a lack of glare was relevant for the determination of the quality of daylight.

"[A warm dwelling] should not be too hot in summer or too cold in winter" Respondent 1 - Study 1, Chile

"For example, in the kitchen. In our old house, we did not cook too much because it was cold and on a cold day we would just eat quickly and go hiding into our room"

Respondent 10 - Study 1, New Zealand

Thus, perception directly influences comfort, and it is clear that it can do so in a negative way. This, however, does not rule out the possibility of perceptions affecting comfort positively, which can happen in at least two ways. The first one is through their effect on people's inferences about the future (which the next section discusses in detail); and the second one, through perceptions that are not limited to "neutrality" in the sense that they can generate pleasure.

One example of perception (or, perhaps, sensation) generating pleasure is alliesthesia (Cabanac, 1971; de Dear, 2011; Parkinson \& de Dear, 2015). That is to say, the fact that, if a certain variable in a person's body has been displaced from its set-point, then returning it to its natural state through external stimuli will generate the feeling of pleasure (de Dear, 2011). For instance, getting into a warm bathtub when feeling cold feels pleasant for a short time. Doing the same when feeling hot may generate the opposite feeling.

Alliesthesia is induced by continuously displacing and restoring internal variables—such as core body temperature - and thus utilizing it to create comfortable dwellings is not always practical. However, it is documented in the literature that, when it is hot, a breeze in naturally ventilated buildings induces pleasure (Candido \& de Dear, 2012; de Dear, 2011). Besides this situation, respondents suggested that, even though the pleasure generated by alliesthesia is only momentary, it might serve as a first impression that lasts longer in people's Feeling of Comfort. Specifically, some respondents mentioned the importance of what people perceive 
or sense when entering a space.

"The feeling of arrival to the house is crucial" ... "You open the door and feel the temperature change and say 'Aaaah, nice, how pleasant"

Respondent 4 - Study 1, Chile

"People often say that you come in on a cold day into the kitchen in the back and [they say] 'this is warm'... it is the light, it is the windows, it is the amount of sunshine, so when it is closed and the sun is coming in, it heats it up"

Respondent 11 - Study 1, New Zealand

It is worth mentioning that the perceptions in Table 6 are not necessarily all different. For instance, most of the perceptions associated with daylight might be, in fact, a single continuous variable/perception that depends only on the brightness (or another indicator) of the space. For example, it is possible that daylight sufficiency-i.e., having enough daylight to be able to operate-is a minimum that, when exceeded, will trigger the perception of Attractiveness of the Space. Likewise, the cleanliness of the space may be a subcomponent of the attractiveness of the space. More research is required for distinguishing properly between these different perceptions. Similarly, it is unclear whether these perceptions depend only on the quantity of daylight (e.g., illuminance or luminance) or if they also depend on contrast and the percentages of direct/diffuse daylight (e.g., Rockcastle et al., 2017; Rockcastle \& Andersen, 2013).

\section{Expected outcomes}

The inferences people make about their future-trying to predict the likelihood of getting involved in certain situations-can influence their assessment of the environment. The concept of Expected outcomes reflects the fact that people are constantly aware of the future and that this awareness partly determines their comfort and their behaviour (although people's behaviour is, strictly speaking, out of the scope of this research).

Table 7: Criteria used to infer expected outcomes identified for daylight and warmness

\begin{tabular}{lll}
\hline \multicolumn{1}{c}{ Warmness } & \multicolumn{1}{c}{ Daylight } \\
\hline - & Passive design & Passive design \\
- Effective and simple systems & \\
- Systems that do not fail & \\
\hline
\end{tabular}

Note: Descriptions of all these elements are available in Appendix IV 
As shown in Table 7, respondents seemed to use three main criteria when inferring what might happen in the future. The best possible future scenario relates to dwellings with a passive design, related to those dwellings that can keep themselves comfortable without the need for people's intervention ${ }^{6}$. Passive design relates to dwellings that will constantly offer a pleasant here and now experience. The availability of effective and simple systems relates to dwellings whose environmental quality can potentially decay but that offer the means for successfully addressing the issue. Similarly, dwellings with systems that do not fail offer some certainty about the future. On the contrary, having unreliable systems reduces comfort regardless of how effective they are when they do not fail.

It can be noticed in Table 7 that respondents did not use the criteria of "effective and simple systems" and "systems that do not fail" when assessing daylight quality. The reason for this is that they consider daylight to be different from electric lighting and thus switching the lights on-while effective and simple and reliable—is not seen as a solution to the lack of daylight.

"Artificial light is different from daylight"

Respondent 13 - Study 1, New Zealand

"I believe that the natural light is more pleasant for living than the fake one"

Respondent 2 - Study 1, Chile

"I much prefer natural light to artificial lighting"... "Daylight is great and it is awesome. I love it!”

Respondent 16 - Study 1, New Zealand

The inferences people make are based on all the elements in the situation, including environmental cues and objective indoor climatic variables (Rohles, 2007), previous experience (Liu et al., 2017; Luo et al., 2016, 2019; Schweiker, Rissetto, et al., 2020) and the adaptive opportunities available. For example, the kind of heating device available in a home or how the roof sounds when it is raining will help infer how easy it will be to keep the dwelling warm.

\footnotetext{
${ }^{6}$ The effect of automatic systems over people's comfort is out of the scope of this research. However, the model introduced in this chapter would predict that different people, i.e., depending on internal elements, will be more or less accepting of this kind of system and thus they will foresee more positive or negative outcomes. In other words, a requisite for a design to be called "passive" is that it must produce satisfactory outcomes. Thus, whether an automatic system is considered to be "passive design" depends on the outcomes that different people think it will produce.
} 
"If you hear all this wind and rain and weather outside, hmmm, it feels closer? ... I like [to feel the rain] as long as I know it is not going to affect me"

Respondent 12 - Study 1, New Zealand

"[A warm dwelling is temperate] without the need for cutting wood and putting it and waiting for it to dry. Or turning the heater on and waiting who knows how long until it is warm... [The heating system] should be very practical and quick"

Respondent 3 - Study 1, Chile

The mechanism through which Expected outcomes affect the Feeling of Comfort is by directly unsettling or soothing people's minds; that is to say, the better the expected outcome, the more comfortable a person will feel. Through this simple mechanism, the model presented here explains the well-known effect of personal control over people's comfort (Lolli, Nocente, \& Grynning, 2020; Luo et al., 2014; Veitch \& Newsham, 2000; Zhou, Ouyang, Zhu, Feng, \& Zhang, 2014). From the perspective of this model, personal control improves people's comfort because, when in control, they know they can fix potentially unpleasant situations. In the opposite case-when there are no effective mechanisms to fix a potentially unpleasant situation-people tend to experience a negative feeling. Thus, when determining the Feeling of Comfort, it is not the control that matters but the expected outcomes. This supports the fact that, in situations where they think automatic systems or other people can deliver better outcomes, people might choose to relinquish control (Burger, 1989).

\section{Trade-offs}

Just like Perceptions reflect an awareness of the here and now and Expected Outcomes reflect an awareness of the future, the concept of Trade-Offs reflects the fact that past choices affect the present. More specifically, choices made in the past might improve certain aspects that determine people's overall subjective satisfaction with a room while deteriorating others. For example, the actions people engage in for improving their satisfaction with one domain (e.g., achieving a neutral thermal sensation by turning the heater on) will often affect other aspects of people's lives (e.g., spending money on heating). These actions result in transactions that do not necessarily improve people's overall experience in the built environment.

Table 8 shows the trade-offs identified as relevant for the domains of daylight and warmness. It is worth mentioning that two kinds of trade-offs can be distinguished in it: those that transfer nuisances within indoor environmental quality domains (e.g., daylight vs 
thermal comfort) and those that affect areas outside of the traditional building science discipline (e.g., thermal comfort vs safety). This implies that respondents tend to put comfort in a much broader context. For instance, people know that thermal comfort is only a part of their overall comfort and that their overall comfort is only a part of their lives. Thus, since people understand that turning a heater on can result in a more acceptable thermal sensation but also increased utility bills, people might sometimes choose to keep the heater off and tolerate cold. In an ideal case, a home should not force people to make these choices.

Table 8: Trade-offs identified for daylight and warmness

\begin{tabular}{|c|c|}
\hline Warmness & Daylight \\
\hline $\begin{array}{l}\text { - Daylight vs thermal comfort } \\
\text { - Utility bills } \\
\text { - } \text { Aesthetics } \\
\text { - Nuisances and lack of safety } \\
\text { - } \text { Thermal comfort vs air quality } \\
\text { - Uncomfortable amount of clothing } \\
\text { - Coolness vs warmness } \\
\text { - Energy efficiency }\end{array}$ & $\begin{array}{l}\text { - Daylight vs thermal comfort } \\
\text { - Utility bills } \\
\text { - Loss of views } \\
\text { - } \quad \text { Increased maintenance and reduced } \\
\text { durability } \\
\text { - } \quad \text { Loss of visual privacy } \\
\text { - } \quad \text { Damage to objects in the house }\end{array}$ \\
\hline
\end{tabular}

Note: Descriptions of all these elements are available in Appendix IV

An example of a Trade-Off was foreshadowed when discussing how some people seem to have a pre-allocated Budget for heating (an internal element, discussed in Section 5.2.1). Specifically, how some respondents expressed that they would simply not use their heatersregardless of the temperature-if that implied high utility bills. Likewise, a common TradeOff seems to be the choice between using an uncomfortable amount of clothing and feeling cold.

"To me, a warm house is one in which, if I want to be barefoot, I can do it without freezing to death" ... "you feel comfortable and do not need to wear too many layers"

Respondent 9 - Study 1, Chile

"[In a warm house] you do not have to wear more than two layers of clothing in the middle of winter"

Respondent 14 - Study 1, New Zealand

"In the cold house... you will need to put a jacket on and put double socks on. Awful." 
Within the literature, evidence of Trade-Offs was documented by Luo et al. (2014). Such a study reports that people who did not have control over the temperature set point in their dwellings tried to avoid thermal discomfort by (1) drinking hot and cold drinks, (2) operating windows, and (3) wearing more or fewer clothes. On the contrary, people who did have control over their temperature set points did not need to rely as much on these three strategies because they could effectively control the indoor environment. In other words, the first group was able to tolerate a wider range of temperatures not because they found them acceptable but because they had no choice other than to exercise the agency they had left. Similarly, Attia (2020) found that, after implementing energy-saving measures in their homes, people often raised the thermostat set point or decided to heat the totality of their dwelling as opposed to only one section. This suggests that, before the intervention, these people were choosing to sacrifice comfort (perhaps due to the potentially high utility bills) and that the retrofit allowed them not to make this sacrifice. In other words, that they could finally heat the totality of the dwelling to their preferred temperature.

Other studies have, from the perspective of the model presented in this chapter, misinterpreted their data because they failed to recognize Trade-Offs. For instance, de Dear et al. (2018, p. 1297) argue that some temperatures "may be regarded by residents as perfectly normal and acceptable in their homes, where usage of heating and cooling equipment comes at a personal financial cost, yet those same temperatures can potentially be deemed unacceptable in the context of the office". From the point of view of the model presented in this chapter, tolerating a wider range of temperatures motivated by the fact that the alternative is to spend an unacceptable amount of money on energy does not reflect a comfortable situation. On the contrary, it reflects a decision to sacrifice comfort in favour of something else. A comfortable home would eliminate the need for this kind of dilemma.

\subsubsection{The Response: the Feeling of Comfort}

The development of comfort is the final stage in the model presented in this chapter. Based on the elements identified from the qualitative data gathered, comfort directly depends on Perceptions, Trade-offs, and Expected Outcomes. In other words, comfort is the state of the mind felt by individuals who:

- Find the bere and now situation to be perfectly pleasant (i.e., perceptions)

- Do not anticipate that they will be involved in unpleasant situations in their future (expected outcomes), and

- Have not made any significant trade-offs to get into the current pleasant situation 
(trade-offs).

A dwelling that meets these three conditions is said to be comfortable, which implies it will offer three main benefits: mental wellbeing, physical wellbeing, and the freedom to maintain a certain lifestyle without the dwelling being a barrier to it. As shown in Table 9, these three components were present in both warmness and daylight.

Table 9: Elements that define comfort for daylight and warmness

\begin{tabular}{lll}
\hline \multicolumn{1}{c}{ Warmness } & \multicolumn{1}{c}{ Daylight } \\
\hline - $\begin{array}{l}\text { Freedom to do what you want wherever } \\
\text { you want } \\
\text { - Mental wellbeing }\end{array}$ & $\bullet \quad \begin{array}{l}\text { Freedom to do what you want wherever } \\
\text { you want } \\
\text { - Physical wellbeing }\end{array}$ & $\bullet \quad$ Mental wellbeing \\
& $\bullet \quad$ Physical wellbeing \\
\hline
\end{tabular}

Note: Descriptions of all these elements are available in Appendix IV

The relationship between warmness and physical wellbeing is relatively straightforward, as warmness is known to reduce the risk of getting respiratory and other medical issues (Howden-Chapman et al., 2005, 2007; Telfar-Barnard et al., 2017). Similarly, daylight is known to improve people's physical wellbeing in at least two ways: encouraging the production of serotonin and Vitamin D (Beute, 2014). Good Physical wellbeing, then, is greatly associated with dwellings that consistently provide beneficial here and now conditions for a living (i.e., perceptions). Respondents seem to know and feel this, as they associated daylight with physical wellbeing through its ability to reduce moisture and mould (by heating the space) and through its capacity of triggering Vitamin D synthesis.

"It is crucial for my children because of a health issue... a warm house allows them to go through the winter better"

Respondent 2 - Study 1, Chile

"You do not get sick in so easily [in a warm house]"

Respondent 14 - Study 1, New Zealand

"[Daylight] gives you life, Vitamin D... what else, lots of things!"

Respondent 15 - Study 1, New Zealand

Similarly, respondents expressed the relationship between daylight quality and mental wellbeing by suggesting that daylight makes them happy and gives them energy, that it makes homes cosier and more liveable, and that not having it is "just depressing". This is consistent with medical findings that suggest that daylight affects people's circadian rhythm 
thus affecting their overall reported wellbeing (Beute \& de Kort, 2014; Cajochen, 2007; Gochenour \& Andersen, 2009; Konis, 2017). This is also consistent with the fact that sunlight patterns and contrast can make a room feel more pleasant, interesting, and exciting (Chamilothori et al., 2019) and with the fact that daylight can have beneficial effects on the stress levels of individuals with depression (Beute \& de Kort, 2018). Likewise, respondents often mentioned that warm dwellings help them not be worried about their children getting sick-which suggest a preoccupation based on Internal Elements-and that they have less initiative when living in cold homes. Thus, not only Perceptions affect people's mental wellbeing, but also the Expected outcomes. That is to say, spaces can feel pleasing here and now, but people's concerns often arise from what they think will happen later. Respondents mentioned this as follows:

"In a cold house, I have less initiative to do stuff inside the house... We are happier now in a warmer house"

Respondent 10 - Study 1, New Zealand

"[Daylight] is good for your body and your soul"

Respondent 8 - Study 1, Chile

"[Daylight] directly impacts your quality of life... Even issues like your mood... It gives you the feeling of liveability, of wanting to be in the house"

Respondent 6 - Study 1, Chile

"[In a warm you do not worry about your children being cold, so it] allows you to sleep well. It is an issue of security and health"

Respondent 1 - Study 1, Chile

Finally, an important benefit of comfortable homes is that they allow people to live their lives and maintain their lifestyle without the opposition of the dwelling itself. When expressing this benefit, respondents often talked in terms of possibilities. That is to say, they want to know that they can do certain things, even if they do not always engage in such behaviour. Consequently, Trade-offs_-which can prevent people from taking actions that would otherwise be considered beneficial—can significantly reduce people's perceived freedom in a dwelling. For example, the participants of this research mentioned the following:

"You can get in and start working right away without the need for, like... I mean, it is already temperate... [there is also] no need for running from one place to another because you freeze" 
"[In our previous house] we decided which room are we going to use today, and that is the room we are going to keep heated"

Respondent 13 - Study 1, New Zealand

"Outside there is a huge deck... so when it is sunny... you can sit outside if you want, enjoy the outdoors... I like to be able to have that option"

Respondent 11 - Study 1, New Zealand

"Your own shadow darkens your view... you are opening a yoghurt and your own shadow bothers you"

Respondent 4 - Study 1, Chile

"If I want to read a book and sit on the sofa, and not having to turn on the lights, that to me is a plus."

Respondent 4 - Study 10, New Zealand

\subsection{Conclusions}

Within building science, the concept of comfort is often introduced as a subjective state of the mind (e.g., Auliciems, 1981, 1983; de Dear et al., 2016). Nevertheless, the models used for assessing it often depend exclusively on physical quantities and on perceptions that can be directly associated with sensing organs in the human body (e.g., Fountain \& Huizenga, 1996; Jakubiec \& Reinhart, 2012; Sarey Khanie et al., 2017; van Hoof et al., 2010; Wienold \& Christoffersen, 2006; Wienold et al., 2019). This is a contradiction: While comfort is defined as subjective, models of comfort avoid dealing with the complexity of the subject by focusing only on physical personal factors (e.g., ASHRAE, 2010). Similarly, while comfort is considered to be a state of the mind, most models ignore cognition as much as possible by directly associating comfort with perceptions that can be treated as if they were objective (e.g., Djongyang et al., 2010; Fanger, 1989; Schweiker et al., 2017).

This chapter utilized the descriptions of a "warm dwelling" and of a "dwelling with good natural light" - data gathered through interviews, as explained in Section 4.4.1- to develop a new conceptualization of comfort - the Feeling of Comfort-and a qualitative model for representing it. The benefit of this development is that it integrates more of the complexity of the subjects and their cognitive processes in a relatively organized way, making it a usable framework for performing comfort research and practice.

The model introduced here does not neglect traditional models of comfort. On the contrary, it acknowledges the relevant role of objective climatic variables in the determination of comfort through sensations and perceptions. However, the new model differs from traditional ones in that it emphasizes the fact that the Feeling of Comfort is a state of the 
mind and thus it is the result of cognitive processes that depend on people's non-physical factors as well as physical ones. Within this new model, the non-physical personal factors are called Internal Elements and include the time in which people occupy their dwellings, their household composition, and the amount of money they are willing to pay in utility bills, among others. Internal elements, along with the elements people can perceive from the physical world, shape the meaning that people give to the different situations they get involved in. In other words, a combination of the physical world and people's Internal Elements will lead—through cognition— to the development of the Feeling of Comfort.

The model of the Feeling of Comfort posits that the cognitive processes utilized by people when assessing a space can be summarized into three main appraisals. The first one is based on Perceptions and is about the here and now. In other words, Perceptions allow people to determine how good or bad the current situation is. On top of that, people are always aware of their future, which means that the Expected outcomes they can think of also influence their Feeling of Comfort. For instance, when people are in control of the environment, they know that they can fix potentially uncomfortable situations and thus they feel more comfortable. This explains the well-known beneficial effect of people's perception of control over their comfort (Hellwig, Schweiker, \& Boerstra, 2020; Lolli et al., 2020; Luo et al., 2014; Veitch \& Newsham, 2000; Zhou et al., 2014).

Finally, people seem to always put comfort into a broader context. They understand that improving their comfort can affect other aspects of their overall wellbeing, and thus the third appraisal that determines people's Feeling of Comfort relates to the Trade-Offs they made to achieve the current situation.

A significant difference between this new concept of comfort and traditional ones is that it does not hold a negative definition. In other words, instead of focusing on what people do not find unpleasant (i.e., in the absence of discomfort), this study focused as much as possible on what people find pleasant (i.e., the existence of comfort). Based on the three appraisals mentioned above, an ideal state of comfort is only achieved when:

- Individuals find the here and now situation to be perfectly pleasant,

- Do not anticipate that they will be involved in unpleasant situations in their future, and

- Had not made any significant trade-offs to get into the current pleasant situation.

Two main implications derive from this new definition of comfort. The first one relates to the fact that people's Feeling of Comfort does not depend exclusively on the here and now. 
On the contrary, it is also determined by what people think will happen in the future andthrough Trade-offs - by the consequences of people's past actions. The second implication is that respondents, contrary to researchers, always put comfort in the broader context of their lives. For example, respondents see thermal comfort as a subcomponent of their comfort, and their comfort as a subcomponent of their overall wellbeing. Consequently, they seem to think holistically when assessing situations and choosing their behaviour (or lack thereof). This not only highlights the importance of performing comfort research more holistically (as opposed to focusing only on single domains), but it also suggests that comfort research should consider elements that are often considered foreign to building science (e.g., privacy, the comfort of clothes, financial concerns, etc.).

The following chapter focuses on assessing how well this model can make sense of data related to other domains of comfort. That is to say, it will check whether people's descriptions of "a dwelling with a good acoustic performance", "a dwelling with good air quality" and "a dwelling that is pleasantly cold (i.e., cool)" can also be understood from the new concept of comfort developed in this chapter. 


\title{
6 ASSESSING THE REPLICABILITY OF THE STUDY
}

\author{
"There are two words in their language on which these people pride themselves, and which they \\ say cannot be translated. Home is the one, by which an Englishman means his house... The \\ other word is comfort; it means all the enjoyments and privileges of home"
}

ROBERT SOUTHEY, LETTERS FROM ENGLAND: BY DON MANUEL ALVAREZ ESPRIELLA (1807) AS CITED BY CROWLEY (1999)

The previous chapter introduced a new understanding of comfort—called the Feeling of Comfort-and a model that represents the process leading to its formation. This model was developed from the data gathered during Study 1 of this research. This means that it is rooted in the respondent's descriptions of dwellings that are "warm" and have "good natural light". This chapter discusses the data gathered through the interviews performed during Study 2 of this research, where respondents described "a pleasantly cold dwelling", "a dwelling with a good acoustic performance" and "a dwelling with good air quality".

The purpose of this chapter is to assess the ability of the model introduced in Chapter 5 to make sense of data beyond that utilized to develop it. In other words, this chapter intends to see whether the model of the Feeling of Comfort is only valid for the domains of daylight and warmness (i.e., those that allowed developing it) or if it also applies to the domains of acoustics, coolness and air quality. This chapter mirrors the structure of Chapter 5, discussing how the model of the Feeling of Comfort can help make sense of the new data, gathered during Study 2 of this research.

\subsection{The Feeling of Comfort}

Section 5.1 argued that, contrary to traditional building scientists, respondents not only expressed comfort in negative terms but also positive ones. The positive connotation of comfort is impossible to capture through models that overemphasize human physiology because equating comfort to a lack of physiological efforts directly implies that the best achievable state is "neutral" (i.e., no effort), and no "good" exists. Despite this, it seems to be that "the mind", through cognitive processes, can give Comfort a positive connotation. 
This was reflected in the descriptions of a warm dwelling and one with good natural light (see Section 5.1) and is also reflected in the descriptions of a dwelling with good air quality, good acoustic performance, and a pleasantly cool home.

For example, respondents expressed how absolute silence-which implies the minimum physiological effort-is not interpreted as a positive situation. On the contrary, positive situations are those that expose people to "good noises". This reflects how comfort and lack of physiological effort can be decoupled and that it is "the mind" that interprets comfort as a positive construct. As the following respondents describe:

"Now I have the option of hearing pleasant noises... Birds... I don't know, now I have a garden, so I can hear the leaves. You have the option of enjoying different sounds"

Respondent 8 - Study 2, Chile

"I love [hearing] the sea"

Respondent 13 - Study 2, Chile

"My evaluation of quiet is 'can you hear the birds?"”... "Quiet, for me, is still, I guess. So like, if there is no noise, it is quiet, it is still, and it is calm"... "I just quite like the quiet. It is calming and I think there is so much noise in our life, figuratively and physically noise, that it is nice to have a quiet, calmed space"

Respondent 16 - Study 2, New Zealand

"We live on a road with just about six houses. And so, it is-and they are very nice people-so it is nice noises. You know, children laughing, people talking in the garden, people playing music."... "Happy noises are good"

Respondent 17 - Study 2, New Zealand

Also, a dwelling that is not excessively hot-i.e., a cool dwelling-produces a sense of satisfaction that goes beyond thermal neutrality. A cool dwelling is not only "not uncomfortable" but it is a place where people actively want to go to. Similarly, respondents mentioned that a dwelling with good air quality is inviting, pleasant and attractive and that the absence of all kinds of smell is not the best situation because there are, indeed, good smells.

"I feel that a [cool] house, comfortable, is also more welcoming. Like, you want to be there, to live there, with more people. It is nice to be [in it]"

Respondent 8 - Study 2, Chile 
"I think that the biggest change is that, when you are outside and there is an infinite number of hells there, you get into the house and it is refreshing. You will want to be there, and it will not be like the oven outside"

Respondent 5 - Study 2, Chile

"[A dwelling with good air quality] is a welcoming house, in the first place... it is a wide concept, but from my point of view, the house should invite you to have a good experience"

Respondent 1 - Study 2, Chile

"Except good smells... It is the smell of oil and other heavy odours that bothers me. Because if I make a cake, I love that smell to be everywhere in the house"

Respondent 2 - Study 2, Chile

Section 5.1 also argued that respondents often expressed comfort as an overwhelming and automatic experience that can be difficult to express in words. Said section also suggested that discomfort seems to be more overwhelming than comfort. The data gathered in Study 2 seems to support these propositions. Specifically, respondents expressed how discomfort produces a strong and overwhelming negative experience:

"My parents' house actually, talking about sound, is horrible... the noise in there is just tedious... I can't stand it"

Respondent 15 - Study 2, New Zealand

"I would rather die. Even if I have armoured windows to stop the noise, I could not live [facing the street]"

Respondent 4 - Study 2, Chile

"I have not lived with children that really make you say 'I truly [need them to shut up]","

Respondent 6 - Study 2, Chile

"I am going to exaggerate a bit, but: [When it is too hot] my head hurts, I want to get out of the place, I want to be outside... So, what can you do? In my apartment, I would be naked spraying myself with water"

Respondent 2 - Study 2, Chile

"We moved into a more modern house and it was just horrible. It was so hot!" Respondent 18 - Study 2, New Zealand 
"You can feel it, right? when you walk in. It is not something you can describe per se, but when you walk in, if the house is too hot, you will feel this heatwave coming or you feel like you can't breathe"

Respondent 19 - Study 2, New Zealand

"I love [Melbourne and Sydney, but they] have multiple days of like 42 degrees. The idea of 42 degrees just freaks me the*\&(\#@\$*\& out. Like, it freaks me out and I still don't know how people survive in there",

Respondent 23 - Study 2, New Zealand

"I am thinking of the experience I have had with covid-19. Like, the other day I went for a vaccine and, afterwards, I felt dirty even if I know I cannot feel the virus... It is something very much related to perception. I think you cannot really feel when the air is dirty... Ifeel like it is purely emotional",

Respondent 2 - Study 2, Chile

"Odours. I have a big issue with them. I mean, I would never in my life purchase an apartment with an open kitchen... I would rather die before having a cushion in the living room smell of onion",

Respondent 13 - Study 2, Chile

Consequently, the Feeling of Comfort associated with warmness and quality of daylight (explained in Section 5.1 and developed from the data gathered in Study 1 of this research) seem to be similar to that associated with acoustic performance, coolness and air quality.

\subsection{Making sense of new data with the Feeling of Comfort model}

As shown in Figure 10, the model of the Feeling of Comfort introduced in Chapter 5 starts from a (1) Situation that needs to capture a person's (2) Attention which will lead to (3) an Appraisal which will cause a (4) Response in the form of a Feeling of Comfort. What remains of this chapter explains how the model developed in the previous chapter also helps to make sense of the data gathered during Study 2-about coolness, air quality and acoustic performance.

\subsubsection{The situation}

Section 5.2.1 introduced The Situation as the starting point of the development of the Feeling of Comfort. It comprises not only objectively measurable climatic variables but also non-measurable environmental cues and people's internal elements. These affect people's Feeling of Comfort through cognitive and physiological mechanisms.

\section{Objective climatic variables}

The data gathered in Study 2 allowed identifying several objective climatic variables 
related to the domains of acoustic, coolness and air quality. These will affect people's Feeling of Comfort through physical/physiological mechanisms. Table 10 shows the objective indoor climatic variables identified as relevant for acoustics, coolness and air quality.

Table 10: Objective indoor climatic factors identified for acoustics, coolness and air quality

\begin{tabular}{|c|c|c|}
\hline Acoustics & Coolness & Air quality \\
\hline - Acoustic absorption & $\begin{array}{ll}\text { - } & \text { Breezes } \\
\text { - } & \text { Air temperature } \\
\text { - } & \text { Relative humidity } \\
\text { - } & \begin{array}{l}\text { Exposure to solar } \\
\text { radiation }\end{array} \\
\text { - } & \begin{array}{l}\text { Floor contact } \\
\text { temperature }\end{array} \\
\text { - } & \begin{array}{l}\text { Mean radiant } \\
\text { temperature }\end{array}\end{array}$ & $\begin{array}{ll}\text { - } & \text { Breezes } \\
\text { - } & \text { Air temperature } \\
\text { - } & \text { Relative humidity } \\
\text { - } & \begin{array}{l}\text { Exposure to solar } \\
\text { radiation }\end{array}\end{array}$ \\
\hline
\end{tabular}

Note: Descriptions of all these elements are available in Appendix IV

For instance, some respondents seem to strongly associate a lack of acoustic absorption with acoustic discomfort:

"[There is an] Asian fusion restaurant, it has a concrete floor and a ceiling that is loud. The space, if it is a busy night, is very loud. I guess, conversely, my experience with this house-which is carpeted and wooden ceilings-is, even if we have a party... sound doesn't reverberate, you can hear things clearly"

Respondent 22 - Study 2, New Zealand

"When there is a lot of people talking-I don't know, 20 people in a relatively small space - do you know how noisy it is?... In general, dwellings are not designed for acoustic performance... I sometimes do not like it when a number of people are talking or screaming"

Respondent 2 - Study 2, Chile

Likewise, respondents mentioned how exposure to solar radiation can significantly affect the perception of coolness in a room, and breezes are understood to help improve a space's air quality.

"We have stopped using the balcony because, since our apartment faces East, the sun strikes over it in the mornings"... "When we got to this apartment, kind of romantic, [we said] 'let's have breakfast in the balcony' [but it was] unbearable. You cannot be there. The sun just gets directly to your face"

Respondent 7 - Study 2, Chile 
"Blocking out the sun sometimes would probably help that too"

Respondent 20 - Study 2, New Zealand

"Ventilation is key, again. An airflow, so you can truly change the air of the house. Like, renew it. I care a lot about this"

Respondent 8 - Study 2, Chile

"Also, when you dry your clothes with the door shut in a room, that is going to attract mould. So, really, drying clothes and stuff should be done outside when possible."

Respondent 16 - Study 2, New Zealand

\section{Environmental cues}

Environmental cues are hard to quantify, but they can affect people's Feeling of Comfort through cognitive mechanisms. That is to say, they help people make inferences about the future and even interpret the here and now, affecting perceptions. The environmental cues identified for acoustics, coolness and air quality are shown in Table 11.

Examples of environmental cues associated with acoustics are the existence of doors between spaces, the proximity to neighbours and the presence of pets. Specifically, being too close to neighbours who have pets implies that people might encounter noisy situations at any moment. Likewise, having good doors between spaces assures people that, if one space becomes noisy, they can isolate themselves from it. Respondents mentioned this as follows:

"When you are in your balcony, no matter the size of it, you would hopefully not face another balcony. Or, your bedroom should not face another balcony, so you can sleep"

Respondent 1 - Study 2, Chile

"I don't want to hear my neighbours. I mean, I am happy sometimes to hear it. You know, I don't need it to be deafening quiet. But I don't really want to hear what they're doing and they don't want to hear what I'm doing'"

Respondent 23 - Study 2, New Zealand

"Far away from the neighbour's dog. Of everything. It must be a big piece of land"

Respondent 13 - Study 2, Chile

"Neighbour's dogs and that kind of thing [don't help] ... it is irritating."

Respondent 24 - Study 2, New Zealand 
"To avoid [bothering my wife], so she sleeps better, I go to the living room when I cannot sleep... I go there to read so I don't bother her. It is good, I think, to have more than one space, at least"

Respondent 7 - Study 2, Chile

"Most houses these days, like new builds, are pretty good. So, double glazed and so, you know, have insulation on the outside. But, the inside is the problem... because all the wooden doors are now, like, pinewood and that sort of stuff"

Respondent 21 - Study 2, New Zealand

Table 11: Environmental cues identified for acoustics, coolness and air quality

\begin{tabular}{|c|c|c|}
\hline Acoustics & Coolness & Air quality \\
\hline $\begin{array}{l}\text { - } \text { Site characteristics } \\
\text { - } \\
\text { - } \\
\text { - } \text { Proximity to neighbours } \\
\text { - } \text { Acoustic insulation } \\
\text { - } \text { Streets nearby } \\
\text { - } \text { Size of the space } \\
\text { - Neighbourhood } \\
\text { - } \begin{array}{l}\text { Nemographics } \\
\text { characteristics }\end{array}\end{array}$ & $\begin{array}{l}\text { - } \text { Orientation } \\
\text { - } \quad \text { Size of the space } \\
\text { - } \quad \text { Heating and cooling } \\
\text { - } \quad \text { Thstem } \\
\text { - } \quad \text { Thermal insulation } \\
\text { - } \quad \text { Affective attributes } \\
\text { - } \quad \text { Materials that feel warm } \\
\text { - } \quad \text { Availability of windows } \\
\text { - } \quad \text { and skylights } \\
\text { - } \quad \text { Floor number of the space }\end{array}$ & 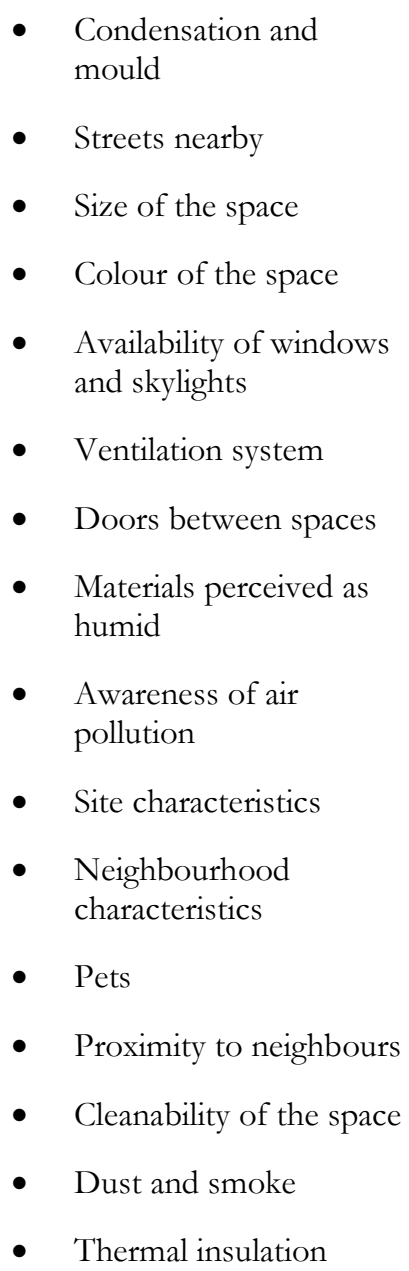 \\
\hline
\end{tabular}

Note: Descriptions of all these elements are available in Appendix IV

Another example of environmental cues, but related to the domain of coolness, is the floor on which an apartment or room is located within the dwelling. Specifically, it would seem that people perceive upper storeys to be hotter than lower ones. While this probably has a physical explanation, there seems to be a direct association between upper 
storeys and excessively hot spaces, as outlined by respondents:

"[The] rooms upstairs are the ones that you need to worry the most if you are looking for a two-storey house"

Respondent 3 - Study 2, Chile

"Our upstairs rooms are a little bit like that. It gets so hot!"

Respondent 18 - Study 2, New Zealand

"The upstairs in some houses. In fact... every house? Heat raises. Upstairs gets hot... I struggle more to keep the upstairs cool than downstairs"

Respondent 20 - Study 2, New Zealand

Similarly, respondents expressed how the availability of windows and/or ventilation systems is a great indicator of what the air quality in a room will be. Depending on the respondent, some systems are thought to be more effective than others:

"Bathrooms for visitors generally do not have windows, they just put an extractor fan. I worry about that [a lot]. That all bathrooms have windows... the bathroom needs, to me, natural ventilation"

Respondent 3 - Study 2, Chile

"I think [HRV] does [help]... It doesn't help in terms of heating, I guess. I mean, some people argue because the air is dryer. But I do think, in terms of mould or something like that, it helps."

Respondent 21 - Study 2, New Zealand

"An extractor fan? No, No. I associate that with a kitchen or a butchery, I don't know... For instance [if you made me choose between] a bathroom with an extraction fan or a window, I choose the window 100\%. The air gets out, you ventilate quickly, the smell goes out. Even if the extractor fan makes it more efficient, I think there is a visual thing there"

Respondent 2 - Study 2, Chile

"All the rooms should have windows so that you can air the rooms. I like opening windows to every room daily. I just like to have some sort of air come through just to air the house"

Respondent 19 - Study 2, New Zealand

It is worth mentioning that affective attributes were mentioned again by a Chilean participant. The explanation is given in Section 5.2.1 (Environmental Cues section) is also valid here. 
"So that every bedroom has access to a garden and you can also go to the living room... also access to a terrace, for instance. It connects both sides of the house [the more public and the more private one] quickly"

Respondent 5 - Study 2, Chile

\section{People's internal elements}

People's internal elements are those that introduce most of the subjectivity to Comfort in the model of the Feeling of Comfort. They are the lens through which people interpret the situation, and thus they have a significant role in the determination of people's Feeling of Comfort. Table 12 shows the internal elements identified in the data gathered in Study 2 of this study.

Table 12: Internal elements identified for acoustics, coolness and air quality

\begin{tabular}{lllll}
\hline \multicolumn{1}{c}{ Acoustics } & Coolness & Air quality \\
\hline - Household composition & $\bullet$ & Household composition & $\bullet$ & Household composition \\
- Lifestyle & $\bullet$ & Lifestyle & $\bullet$ & Lifestyle \\
- Dwelling typology & $\bullet$ & Thermal preference & $\bullet$ & Sensitivity to odours \\
- Times of occupancy & $\bullet$ & Times of occupancy \\
- Love for music & & & \\
$\bullet \quad$ Sensitivity to noise & & & \\
\hline
\end{tabular}

Note: Descriptions of all these elements are available in Appendix IV

Probably the most important internal element identified in this research-because it was already present in the domain of warmness and was also found in the three domains covered by this chapter-is people's household composition. Participants below reflect on the fact that people do not only care about themselves but also about other people who live with them.

"[A house with good acoustic performance] has no kids... and if there are kids, it is, probably, reasonably big. As, even if you have good insulation or whatever to stop the noise, our kids are pretty loud, so you need them faraway"

Respondent 14 - Study 2, New Zealand

"It is a bigger issue in my case because... my older son has [some] sensorial issues. He is hypersensitive to certain noises... so, he needs environments that are as homogeneous as possible in terms of noise"

Respondent 8 - Study 2, Chile 
"Children can nap properly. Without... waking up due to the heat, which happens with [babies]. That happened to us in our previous apartment, with [my son], he woke up due to heat and slept worse during summer because of that... Children's naps, very important. Write that down!"

Respondent 10 - Study 2, Chile

"I don't care so much about the dust and stuff, although I imagine if I had children or a spouse that is asthmatic or something, I probably would be more concerned"

Respondent 15 - Study 2, New Zealand

"Now, if I have [trees that produce allergies] outside my balcony, I will say no [to that place]. Because that affects my air quality because we are very allergic. [My son] too... [He] is asthmatic because of allergies",

Respondent 10 - Study 2, Chile

Also, just like in Study 1, people who are searching for a new home will utilize different elements to infer how comfortable the dwelling is, depending on the typology of the dwelling they are looking for.

"When you are in a place where there are more people, you feel the noises more. In apartments, this is quite notorious."

Respondent 8 - Study 2, Chile

"It doesn't have to be standalone [to have a good acoustic performance]... [If] it has like neighbours [and] good walls in between the two, you don't hear your neighbours so much"

Respondent 20 - Study 2, New Zealand

\subsubsection{The attention}

Three main propositions related to attention were derived from Study 1 (Section 5.2.2). First, it was suggested that people are less likely to attend to situations in which the environmental conditions produce neutral perceptions. Second, that relatively abrupt changes in the environmental conditions can force attention, even if the change ultimately offers a more beneficial situation. And third, that situations can capture a person's attention not only through physical/physiological mechanisms but also psychological ones. Similar patterns seem to be reflected in the data gathered in Study 2.

For instance, respondents mentioned again that they were not always attending to the situations they are involved in and that, apparently, situations that produce non-neutral perceptions are more likely to capture people's attention than others. 
"I have never even noticed [my neighbours], so yea, they are quiet"

Respondent 15 - Study 2, New Zealand

"I do not hear my neighbours often [in my current apartment]. I am actually not sure if I have neighbours downstairs. I don't think so, I have never known, but you can barely hear anything"

Respondent 9 - Study 2, Chile

"There was a guy who went to [a university close by] in his skateboard, and I heard him. It was unbearable"

$$
\text { Respondent } 7 \text { - Study 2, Chile }
$$

"With respect to odours, I have a bad sense of smell. Thus, the truth is that this is not a driver for me at all."

Respondent 6-Study 2, Chile

"I would want to avoid the nuisance from smells from the city around me. Like cars exhaust and smoke and stuff. I wouldn't want to smell anything from outside except fresh air, if I could keep that in."

Respondent 18 - Study 2, New Zealand

And again, relatively abrupt changes in environmental conditions can make people attend to a situation, even if these changes are beneficial. Respondents mentioned that these changes can be caused by moving in or out of a room, but also by breezes, sudden noises, and others.

"It was a little bit stale when we came in, so I really wanted to get that kind of airflow moving and, does that count as air quality?"

Respondent 23 - Study 2, New Zealand

"in a cooler house, where there are some breezes, I immediately say 'How nice, clean air"'

Respondent 2 - Study 2, Chile

"It is an unpleasant noise because it is random. It works at 2 am during the week, at 3 am... those noises bother me"

Respondent 3 - Study 2, Chile

"Neighbour's dogs and that kind of thing [don't help]... It is irritating. In my current flat, there's a neighbour next door who laughs very loudly all the time and has a dog outside most of the day, barking and yapping."

Respondent 24 - Study 2, New Zealand 
"I think that the biggest change is that, when you are outside and there is an infinite number of hells there, you get into the house and it is refreshing."

Respondent 5 - Study 2, Chile

"You can feel it, right? when you walk in. It is not something you can describe per se, but when you walk in, if the house is too hot, you will feel this heat wave coming or you feel like you can't breathe"

Respondent 19 - Study 2, New Zealand

Finally, this study seems to confirm that the reasons to attend to a situation are not necessarily physiological/physical but also psychological. For instance, environmental cues and the awareness caused by the media can induce attention towards air quality. Similarly, the meaning that different sounds represent to people (as stated by Angevine, 1972) and the concern of bothering others can make people attend to situations. Respondents expressed this as follows:

"I think you cannot really feel when the air is dirty. Like in Santiago, I am thinking about the smog. On the contrary, there are two things that will condition you... You read the newspaper and you are told that your neighbourhood has very polluted air... and looking outside and seeing [pollution, smog] on the horizon, you cannot see the hills"

Respondent 2 - Study 2, Chile

"My neighbour in my previous flat, he was quite worried about that we lived relatively close to a main road. He was one house closer in. One of the reasons he said he was moving was because, when he changed the air filter in their air conditioner or heat pump or whatever it was, it was quite black... And I hadn't even noticed an air quality issue, whereas he was quite particular about the air quality at his house. And I don't know whether it was that difference or just him being 50 meters closer to that main road was meaning he was getting a lot of vehicle fumes that we weren't. Maybe it had dissipated by the time it got to us."

Respondent 23 - Study 2, New Zealand

"In our previous apartment, which was a much bigger building [in which] we had neighbours in every direction, sometimes [my son] would not stop crying and you said... 'they are truly going to ask us to leave the apartment'. Because they could hear"

Respondent 10 - Study 2, Chile

"[but birds make noises] Yeah but, that is hmmm, yeah to me that is quiet, though."

Respondent 16 - Study 2, New Zealand 
"I worry [about my children], but they do not suffer [when it is too hot]. You know what happens? You put them to bed in summer and then you find them at 11 pm all sweat. So, they catch a cold later"

Respondent 3 - Study 2, Chile

\subsubsection{The appraisal}

The appraisal stage is when people give meaning to a situation they are already attending to. As stated in Section 5.2.3, this stage introduced the largest theoretical difference between the model of the Feeling of Comfort and traditional Comfort models because it reflects the fact that not only here and now Perceptions affect people's Feeling of Comfort. On the contrary, the model introduced in Chapter 5 posits that people's Feeling of Comfort also depends on what they think will happen later (i.e., Expected Outcomes) and the sacrifices they made to achieve their current situation (i.e., Trade-offs). This section discusses the Perceptions, Trade-offs and Expected Outcomes identified for the domains of acoustics, coolness and air quality.

\section{Perceptions}

Perceptions are how people interpret their current situation. They are people's interpretations of all the stimuli they receive from the environment. Perception's here-and-now nature implies that they represent the influence of the present over peoples' comfort (as opposed to expected outcomes, which represent the influence of the future over people's comfort). They indicate to people whether the immediate environment is acceptable or not.

As mentioned in Section 5.2.3, building science's comfort research has for years acknowledged the role of perceptions. However, most of the studied perceptions are directly relatable to sensing organs and thus are sensation-driven (e.g., loudness relates to people's ears, and glare to their eyes). Section 5.2.3 also explains how the data gathered in Study 1 suggested the existence and importance of perceptions that are more cognition-driven and that do not directly relate to sensing organs. For instance, respondents expressed how a space with good daylight reduced people's feeling of confinement.

Just as in the case of warmness and daylight, the perceptions identified for the domains of acoustics, coolness and air quality are not all directly relatable to sensing organs and they often involve a large cognitive component. For example, the perception of bothering others (associated with acoustics and air quality) is completely foreign to people's own bodies, and thus it can only affect their comfort through cognitive mechanisms. Table 13 shows the Perceptions identified for the domains of acoustics, coolness and air quality. 


\begin{tabular}{|c|c|c|}
\hline Acoustics & Coolness & Air quality \\
\hline $\begin{array}{l}\text { - } \quad \text { Loudness vs origin } \\
\text { - } \text { Bothering others } \\
\text { - } \text { Acoustic privacy } \\
\text { - } \quad \text { The sound of silence } \\
\text { - } \quad \text { Sense of community }\end{array}$ & $\begin{array}{l}\text { - } \quad \text { Daylight sufficiency } \\
\text { - } \quad \text { Thermal sensation } \\
\text { - } \quad \text { Sensation when entering } \\
\text { a space } \\
\text { - } \quad \text { Feeling of confinement } \\
\text { - } \quad \text { Cleanliness of the space }\end{array}$ & $\begin{array}{l}\text { - } \text { Feeling of confinement } \\
\text { - } \text { Cleanliness of the space } \\
\text { - } \text { Daylight sufficiency } \\
\text { - } \quad \text { Thermal sensation } \\
\text { - Odours }\end{array}$ \\
\hline
\end{tabular}

Note: Descriptions of all these elements are available in Appendix IV

Another example of a cognitive-driven perception-even if it has historically been strongly associated with physiology — is loudness. Specifically, respondents tended to always express loudness attached to the origin of the sound, suggesting that the same level of sound might be acceptable or not depending on its source and the meaning given to it. For instance, the noise heard from the toilet is not necessarily loud, and yet respondents seemed to dislike it. Also, respondents expressed that the noise from traffic and cars is very annoying and that noises from nature are pleasant as long as they do not suggest any danger:

"Sometimes you hear everything that happens in the toilet. It might be a bit inevitable, but I have been in places where that does not happen"

Respondent 1 - Study 2, Chile

"Maybe [I can stand] natural noises. 'Some' natural noises, and constant noises. But human noises, not that much."... "I also dislike... for instance, in my current house... everything creaks"... "I tend to associate them with wear and tear. Like the house is old"

Respondent 2 - Study 2, Chile

"Like, when you hear the trees blowing in the wind, regardless of where you are, you know that is creating sound in your home... I don't mind it, but the thing is that, when you hear stuff like that, you start to think about, like, what the weather is doing and start to panic... But, I would much rather hear that than ... the motorway traffic. It is a much easier noise to be able to relax in vs hearing cars and trucks"

Respondent 15 - Study 2, New Zealand

Just as in the case of warmness, respondents seemed to positively remember the sensation of entering a cool space on hot days. At first sight, this can be associated with alliesthesia (Cabanac, 1971; de Dear, 2011; Parkinson \& de Dear, 2015). However, it would seem that some respondents remembered this sensation beyond the brief time in which they 
felt it, suggesting that the moment of entering a space can influence how people assess spaces. In other words, even if alliesthesia is a brief sensation, the liberating experience of entering a comfortable space after being in an uncomfortable exterior environment seems to leave a more permanent impression that changes people's assessment of the built environment. For example, some respondents said:

"My parent's former house was very cool. In terms of temperature and also in the sense of feeling comfortable when getting in there"

Respondent 7 - Study 2, Chile

"We have found it crucial to have air conditioning... We get home, outside it is $37^{\circ} \mathrm{C} \ldots$ and you have a wave of cold air. I swear it changes your whole life!"

Respondent 4 - Study 2, Chile

"When you walk in, it should feel comfortable... It shouldn't be this wave of hot air that heats you. You shouldn't get a 'heatwave thing' through",

Respondent 19 - Study 2, New Zealand

Respondents also mentioned how their thermal sensation can affect the perceived quality of the air. Specifically, it would seem that some people associate hot air with dirty air; and cold air with clean air. Nevertheless — when combined with other stimuli (e.g., humidity and moisture) — cold air can also be associated with mould and poor air quality.

"I associate, I don't know why, heat with more polluted air. Maybe because you feel a bit suffocated... The experience of a relatively cool house makes you feel like you are breathing cleaner air, even if it is the same air"

Respondent 2 - Study 2, Chile

"I strongly associate clean air with cool air."

Respondent 4 - Study 2, Chile

"You can feel the coldness. When I was house-hunting, one of the things that I would roll out a house for was that [it] felt cold. Like, it didn't feel cold as if... it felt wet, it felt like it was a damp home"

Respondent 15 - Study 2, New Zealand

\section{Expected outcomes}

People are always aware of their future, and thus the actions they engage in are always taking into account what they think will happen later. These Expected Outcomes can influence people's Feeling of Comfort through cognitive mechanisms. Section 5.2.3 outlined three main criteria people utilized by respondents when inferring what might happen in the 
future. These three criteria were also mentioned by respondents of Study 2 and correspond to passive design (i.e., the dwelling will keep itself comfortable without any intervention), effective and simple systems (i.e., if the dwelling becomes uncomfortable it can be solved), and systems that do not fail (i.e., the systems in place will not stop working anytime soon).

Apart from the three shared criteria identified in both Study 1 and 2, the latter revealed a new criterion utilized by respondents for inferring the future: the fact that a nuisance is temporary. Respondents associated this criterion to situations in which the dwelling does not have a "passive design" and it offers no "effective and simple systems" to solve discomfort, forcing them to simply hope that the nuisances finish over. While absent from the data, it is possible to imagine how this criterion would be useful when people expect to be exposed to nuisances only for a short time (e.g., when briefly entering a room to pick up something), in which case they might decide not to take action. Table 14 shows the criteria identified for the domains of acoustics, coolness and air quality.

Table 14: Criteria used to infer expected outcomes for acoustics, coolness and air quality

\begin{tabular}{|c|c|c|}
\hline Acoustics & Coolness & Air quality \\
\hline - $\quad$ Passive design & - $\quad$ Passive design & - $\quad$ Passive design \\
\hline $\begin{array}{l}\text { Effective and simple } \\
\text { systems }\end{array}$ & $\begin{array}{l}\text { - Effective and simple } \\
\text { systems }\end{array}$ & $\begin{array}{l}\text { - Effective and simple } \\
\text { systems }\end{array}$ \\
\hline - Systems that do not fail & & - Temporary \\
\hline - Temporary & & \\
\hline
\end{tabular}

Note: Descriptions of all these elements are available in Appendix IV

The reason this new criterion-that a nuisance is temporary-was mentioned in the quotes below within the domains of acoustics and air quality probably relates to the fact that they are, generally, the least controllable. This happens because nuisances related to these domains—e.g., noise and odours—can come from neighbours or the street.

"That noise is annoying because, at least, you know that a party will be over at some point. But this one is constant. If you move any furniture upstairs, we can hear it"

Respondent 3 - Study 2, Chile

"We now have a construction in front of us. That has been noisier, but I know it is temporary. So I do not [take it] too much into consideration. It has been quite unpleasant sometimes, but there is not so much you can do about it"

Respondent 6 - Study 2, Chile 
"We are having buildings works done... so there is quite a bit of dust at the moment, but normally it is not a dusty house... it is just minor work"

Respondent 17 - Study 2, New Zealand

"Smell and things probably come down to just hygiene of the house... Houses always have a smell of the people that would be living in them... I feel like, that is something that would change. So if you buy a house and it has a certain smell of the people who were living in there before, you clean it... I do not think that is a big deal... But if I find like a bad smell, like, that might be the result of mould or something like that ... that is something that you would have to address"

Respondent 16 - Study 2, New Zealand

\section{Trade-offs}

Trade-offs reflect how improving one aspect of people's lives can deteriorate other aspects and thus have no benefit over people's overall wellbeing. There are at least two kinds of Trade-offs. The first one comprises those trade-offs that reflect transactions between subdomains of comfort; for instance, opening windows might cool down a room sometimes, often at the cost of allowing noise to come in. The second group of trade-offs comprises transactions between comfort domains and areas that are not part of traditional building science; for instance, opening windows to cool down a room might expose people to dangers they are not willing to take. As shown in

, this same pattern can be identified from the data gathered in Study 2, relating to acoustics, coolness and air quality.

One trade-off that was identified for the domain of warmness and that repeats itself in the domain of coolness and air quality is called nuisances and lack of safety. This Tradeoff represents circumstances in which, even if people have potentially effective systems for improving their environmental quality in one domain, they hesitate to use them due to their negative consequences on other areas of their lives. For example-within the warmness domain - one respondent mentioned that some heating systems could put elderly people at risk of burning themselves. In the case of air quality, some respondents expressed that heat pumps excessively reduce the relative humidity of the air and that some systems can reduce air quality. In the case of coolness, on the other hand, respondents mentioned this kind of Trade-off when expressing that some swimming pools (seen as a system to cope with heat) are often too cold to be pleasant, that fans are too noisy, and that air conditioning makes them sick. 


\begin{tabular}{|c|c|c|}
\hline Acoustics & Coolness & Air quality \\
\hline $\begin{array}{l}\text { - } \begin{array}{l}\text { Air quality vs acoustic } \\
\text { performance }\end{array} \\
\text { - } \quad \begin{array}{l}\text { Thermal comfort vs } \\
\text { acoustic performance }\end{array} \\
\text { - } \quad \begin{array}{l}\text { Complete silence vs } \\
\text { sound of nature }\end{array} \\
\text { - } \quad \text { Loss of views } \\
\text { - } \text { Openness of the space } \\
\text { - Safety } \\
\text { Location }\end{array}$ & 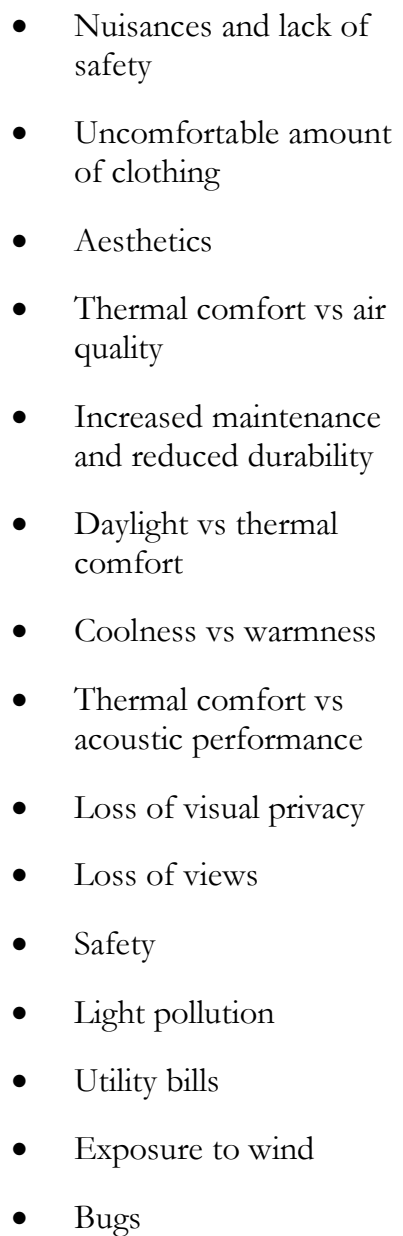 & 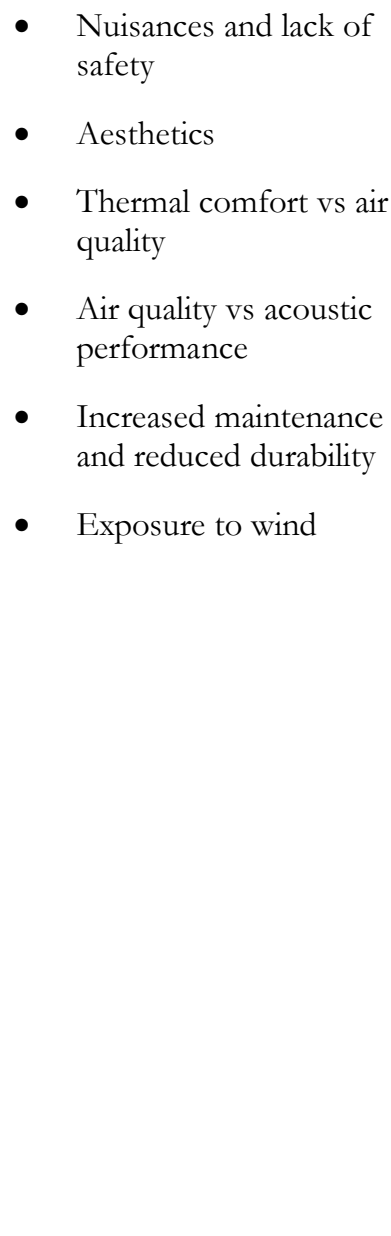 \\
\hline
\end{tabular}

Note: Descriptions of all these elements are available in Appendix IV

As the following respondents explain:

"We have got a heat pump here as well. And I think that that is supposed to help. But I don't really like the heat pump air that much... it is just dry. Too dry"

Respondent 19 - Study 2, New Zealand

"Air conditioning, which blows air, for me is not air quality. On the contrary, I associate it with poor air quality, with something recycled. They sometimes leak, produce humidity"

Respondent 2 - Study 2, Chile

"I know there are a lot of, some people, who are not fans of the HRV systems because they worry about the filters and the air quality in the roof"

Respondent 20 - Study 2, New Zealand 
"[My wife] uses my parent's pool every time we go there and she never uses this one because it is cold."

Respondent 3 - Study 2, Chile

"[I would rather not use air conditioning] because it makes me sick, it gives me a cold... my wife likes air conditioning, but I do not. In fact, I even try not to use it in my car."

Respondent 9 - Study 2, Chile

"[Fans work fine] but they're quite noisy... for example... I like to sleep with the fan, but then [my partner] can't sleep because of the noise of the fan. So, then he turns it off and then I wake up in the middle of the night like sweating"

Respondent 23 - Study 2, New Zealand

\subsubsection{The Response: the Feeling of Comfort}

Section 5.2.4 described the Feeling of Comfort as a state of the mind felt by individuals who:

- Find the bere and now situation to be pleasant (i.e., perceptions)

- Do not anticipate that they will be involved in unpleasant situations in their future (expected outcomes), and

- Have not made any significant trade-offs to get into the current pleasant situation (trade-offs).

Then, that section mentioned that dwellings that meet these three conditions would offer three benefits: mental wellbeing, physical wellbeing, and the freedom to do what people want wherever they want. These three benefits were shared by both the warmness and daylight domains and, as shown in Table 16, also by the domains of acoustics, coolness and air quality. The only exception is the domain of acoustics, which does not seem to offer physical wellbeing. The cause of this exception probably relates to the fact that the noises to which people are exposed to without their consent are not loud enough to produce any physical harm (Cowan, 1994, p. ix). 
Table 16: Elements that define comfort for acoustics, coolness and air quality

\begin{tabular}{|c|c|c|}
\hline Acoustics & Coolness & Air quality \\
\hline $\begin{array}{l}\text { - Freedom to do what } \\
\text { you want wherever you } \\
\text { want }\end{array}$ & $\begin{array}{l}\text { Freedom to do what } \\
\text { you want wherever you } \\
\text { want }\end{array}$ & $\begin{array}{l}\text { - Freedom to do what } \\
\text { you want wherever you } \\
\text { want }\end{array}$ \\
\hline \multirow[t]{2}{*}{ - Mental wellbeing } & - $\quad$ Mental wellbeing & - Mental wellbeing \\
\hline & - $\quad$ Physical wellbeing & - $\quad$ Physical wellbeing \\
\hline
\end{tabular}

Note: Descriptions of all these elements are available in Appendix IV

Even if they did not mention it when describing a dwelling with a good acoustic performance, respondents below expressed how cool dwellings and those with good air quality allow them to avoid suffocation and sweating without the need for unnatural and unhealthy air conditioning systems. Additionally, dwellings with good air quality allow people to avoid dust, allergies, mould, and dampness.

"I also worry that, when you start putting, like, heat pumps and air conditioning things on, that your air is not quite the same. It is not quite natural and your body can get sicker. I find that when you change the temperatures too much"

Respondent 15 - Study 2, New Zealand

"[Children] should not feel cold, but they should not sweat either. Looking for that intermediate point is more difficult"

Respondent 8 - Study 2, Chile

"You feel suffocated [by the heat]. You do not know where to move to, I feel" Respondent 13 - Study 2, Chile

"[Dust] implies allergies, implies a series of nuisances. Here, dust is annoying"

Respondent 7 - Study 2, Chile

"I don't think [dust] is very good for your lungs. Plus I am a compulsive tidier and everything is cleaned in this house like every day"

Respondent 18 - Study 2, New Zealand

"Here I have a sliding door, and one of those air extractor fans, but the shower is too powerful. So I have to shower with the door open because, truly, Ifeel asphyxiated"

Respondent 4 - Study 2, Chile

Respondents expressed the relationship between the freedom to do what you want 
wherever you want and acoustics mainly in terms of acoustically separating spaces, allowing people in one area to do things without bothering other people or being bothered. Similarly, respondents expressed their desire of being able to sleep and work without noise being a problem. In terms of coolness, respondents expressed how everything becomes harder and unpleasant when the temperatures are excessively high. Some spaces become unusable, sleeping becomes difficult, and the lifestyle needs to be adapted to use the right areas of the dwellings at the right moment of the day and year. Finally, within the domain of air quality, respondents focused on the fact that — when a dwelling had good performance-they were able to cook without fearing the consequences. All this is summarized by the following quotes:

"I would not like to be in the kitchen, using some appliance, and that the noise can be heard everywhere"... "It is, really, a nuisance for the rest. Hopefully what I do, which I enjoy doing, [does not bother the rest of the people]"

Respondent 5 - Study 2, Chile

'My parents' house actually, talking about sound, is horrible. So, in the kitchen/living space, the TV has to be so loud to be able to hear it properly, that in the kitchen (which is part of that space) it is soloud. And so you are always in the kitchen telling the person watching TV to turn it down"

Respondent 15 - Study 2, New Zealand

"Now, for instance, when experiencing home office, it has been uncomfortable for Zoom meetings. You hear the cars outside, you cannot hear well."... "If it is a noisy house, you cannot sleep. I mean, it gets really hard to do it"

Respondent 11 - Study 2, Chile

"As I get older and I am finding [that] background noises-of the kitchen and stuff like that-[makes it] harder to hear the TV"... "I work in an office where the tenant had no insulation... It's an old warehouse building and I find the, you know, when it's heavily raining you just can't hear anything",

Respondent 23 - Study 2, New Zealand

"If you did have kids, then you'd want the ability to still have other people around at your house without needing to worry that your kids will wake up, or partners, or whatever"

Respondent 24 - Study 2, New Zealand

"A cool house, just to summarize, allows you to be in your home whenever you want to be in it... sometimes you are forced to go out, and you find that that the heat is worse outside."

Respondent 1 - Study 2, Chile 
"[When it is hot] you do not want to do the stuffyou have to do in your home... spend time with your family, cook, clean. Like, daily activities that we practice in our house, a hot house makes it much more unpleasant. So, in the end, you spend the whole day on a sofa, with a fan, hopefully. Or next to a window. You would never think of cooking... although you need to do it anyway "

Respondent 11 - Study 2, Chile

"It is a limitation. I mean, you do not want to be in that place on a Sunday or Saturday morning. But well, you can simply avoid using the balcony at that time... in the end, we still use with no problem after $12 \mathrm{pm}$ "

Respondent 7 - Study 2, Chile

"If you are going to cook something stinky and you will bother all your neighbours, you might not do it. But, for me, no. The worst that can happen is that you need to open the windows and in five minutes it is all forgotten"

Respondent 7 - Study 2, Chile

"Also having a good extractor fan in the kitchen. You know, because we make lots of cups of tea... [we also have] one really large window and one small window"

Respondent 17 - Study 2, New Zealand

The relationship between Mental wellbeing and air Quality was expressed in terms of respondents' concerns with regards to the durability of the dwelling (i.e., rotting) and also in terms of how welcoming the dwelling is.

"I am actually more concerned about my house rather than my health. I don't want my house to deteriorate... I care about my health too, but if my home is damp and causes issues (e.g., rot) then that is potentially a high cost and that is of more concern to me. It actually comes back to my own health, as if there are issues caused by having a damp home, then I will stress about it (mainly to do with financial costs of repairs)"

Respondent 15 - Study 2, New Zealand

"It is a cosy house, in the first place... it is a wide concept, but from my point of view, the house should invite you to have a good experience"

Respondent 1 - Study 2, Chile

The relationship between mental wellbeing and both the acoustic and the coolness domains, on its part, relates mostly to the extreme feelings they can produce. On the one hand, there is peace and calm; and on the other, there is an overwhelmingly stressful experience that affects not only the individual but extends to other members of the household as well: 
"[Noise is] my enemy number 2 [the first one is heat]"... "I cannot stand the street"... "The other day I went to a friend's apartment, and I could not believe it. I tried to make [my daughter] sleep and it was impossible. You did not even know whether the windows were open or not due to the noise outside"

Respondent 1 - Study 2, Chile

"[Quiet] gives you tranquillity, peace"

Respondent 13 - Study 2, Chile

"This house... you can hear absolutely everything, which is not good. Hmm, and I really hate that."

Respondent 18 - Study 2, New Zealand

"High temperatures destroy you when you are trying to sleep at night"

Respondent 3 - Study 2, Chile

"For me, the worst that can happen is to suffer from the heat. To sweat. You feel sticky, I don't know. A series of unpleasant things. It is a totally personal and subjective feeling. I, generally, like it cold"

Respondent 7 - Study 2, Chile

\subsection{Conclusions}

Chapter 5 introduced a qualitative model representing the Feeling of Comfort, developed from how respondents—interviewed during Study 1 of this research—described a warm dwelling and a dwelling with good daylight. The purpose of this chapter was to assess whether the model introduced in Chapter 5 was limited to the domains that allowed to develop it, or if it could also be applied to other domains. The main conclusion of this chapter is that the Feeling of Comfort model can explain the data gathered during Study 2 of this research—about coolness, acoustic performance and air quality—without the need for any significant changes. These results further support the definition of Comfort and the Feeling of Comfort model introduced by Chapter 5 .

The only modification to the model introduced in Chapter 5 was the addition of a new criterion people use for inferring the future, associated with the domains of acoustic and air quality. This criterion consists of hoping or focusing on the fact that a certain unpleasant situation is only temporary. The reason for this association is speculated to be that the nuisances related to these two domains-e.g., odours, smoke and noise-are often originated by neighbours, which cannot be controlled and thus people are left helpless. Apart from this addition, the whole dataset of this study seemed to fit within the Feeling of Comfort. 


\section{A MATHEMATICAL MODEL OF THE FEELING OF COMFORT IN RESIDENTIAL ENVIRONMENTS}

"I am old enough to know that you can never say for sure what someone else will do"

LAGERTHA, VIKINGS

The past two chapters proposed a new qualitative model of comfort that emphasizes the fact that comfort is a state of the mind-a model of the Feeling of Comfort. This model emphasizes that people's cognitive processes and non-physical attributes play an important role in the determination of people's comfort. This chapter proposes a mathematical representation of the Feeling of Comfort which building performance simulation tools can incorporate. The value of doing this is that this kind of tool is the standard tool utilized for evaluating building designs and estimating the performance of buildings (Ayoub, 2019; Crawley, Hand, Kummert, \& Griffith, 2008; Mazzarella \& Pasini, 2009).

The mathematical representation presented in this chapter is the first part of a direct response to Research Question 3 introduced in Section 3.2-i.e., how can predictive models of comfort incorporate people's non-physical characteristics and their cognitive processes? (Chapter 8 contains the second part of the response.) It is worth mentioning that the mathematical representation introduced in this chapter does not pretend to be a fully finished mathematical model but to express some ways in which the Feeling of Comfort can be modelled mathematically.

Thus, this chapter introduces a set of mathematical equations that can help represent the Feeling of Comfort. However, before presenting all these equations, this chapter narrows down the scope of the model proposed in Chapter 5, making it applicable only to people's own homes or other highly familiar environments. Doing this allows significant simplification of the problem without the need for completely disregarding "the mind". 


\subsection{The Feeling of Comfort at home}

The model introduced in Chapter 5 includes three elements that are part of what this thesis has called "the mind". First, people's Internal Elements; in other words, their nonphysical personal factors. Second, Trade-Offs; which are the sacrifices people made to achieve the current level of comfort. And third, Expected Outcomes; reflecting what people think will happen to them later. Trying to develop a general mathematical model of this third component is a challenging task. However, if the scope of the model is limited to environments that are highly familiar to people_-such as their homes_estimating what people think will happen becomes much easier because people's predictions are based on their vast experience in those environments as opposed to wild guesses.

As explained in Sections 2.4.2 and 2.4.3, people's intuitive predictions about the built environment are likely to be accurate (especially when their predictions are about building they know well). For instance, Schweiker, Rissetto and Wagner (2020) found that people's thermal expectations are fulfilled the majority of the time, which suggests that people can read environmental cues to make relatively accurate inferences about it. Likewise, research about long-term comfort or performance sometimes relies on people's knowledge of how buildings they are familiar with behave (e.g., Jakubiec \& Reinhart, 2015; Reinhart, 2014, p. 97). Additionally, Kahneman and Klein (2009) posit that intuition can be trusted when trained through prolonged practice in environments in which there is a stable relationship between objectively identifiable cues and subsequent events. Since people spend a great portion of their lives at home, it is possible to state that their intuition can be trusted when talking about how their own home behaves. Participants of this research, for instance, described the behaviour of their dwellings in great detail:

"There is a heat pump that was previously there, which is in the main room and the lounge, which is astonishingly effective"

Respondent 11 - Study 1, New Zealand

"I think West is unbearable in summer and in winter it is not good at all... My [previous] apartment had a very unpleasant section facing West"

Respondent 2 - Study 1, Chile

"In my current house it is, it can be, cold when I wake up, but I have got a central heating thing. So, if I want to take a cup of tea and then go back to bed and read the newspaper, it is warm enough. It is as warm in my room as anywhere else in the house" 
"The house that I am renting now, for instance, has a south-facing dining room, and... I turn the lights on even for having lunch. I do not have enough light and that is quite unpleasant"

Respondent 1 - Study 1, Chile

"[In my kitchen, which is dark] you need to turn the lights on much earlier. If in a bedroom you need to turn the lights on at 19:00, here you need to do it at 17:30"

Respondent 4 - Study 1, Chile

"I live in an apartment built in 2018, so it has double glazing and I never hear anything. I mean, you hear it, but feels like it is in the distance. I can sleep. If someone starts drilling two blocks away, I might hear it, but it is [not an issue]. Also, here I am one block away from [a very big avenue], which in general is quite noisy... [but] you almost cannot hear a thing. You would obviously hear the sirens and horns but like far away"

Respondent 4 - Study 2, Chile

"The [dance] studio is directly below this room. And you can hear it echoing up through...[the] bathroom ... it is not as easy to hear [that noise] when that door is shut, but you can hear it - when that door is open-really hardly."

Respondent 18 - Study 2, New Zealand

"In Santiago, the breeze starts blowing in the evening"

Respondent 1 - Study 2, Chile

"That happens to me in my current house... in fact, it is often a bit cold... it is hard to temperate"

Respondent 4 - Study 2, Chile

The fact that people's inferences of the future are likely to be accurate leads to the first assumption and the first restriction of the mathematical model presented in this chapter. Specifically, the expected outcomes can be approximated by estimations of what will happen in the built environment. This assumption only applies to environments that are familiar to the subject whose Feeling of Comfort is being estimated. While this can be seen as a limitation, it considerably reduces the complexity of the problem because the mathematical models can now focus on well-known and quantifiable building physics (i.e., what will happen) as opposed to arguably obscure estimations based on non-quantifiable factors.

Having incorporated the role of Environmental Cues in the development of Expected Outcomes (through the approximation just mentioned), it becomes necessary to account for their effect on people's perceptions. For instance, to account for the fact that the appearance 
of a room can affect people's thermal sensation (Rohles, 2007) or that thermal sensation can be affected by the colour of the light (Chinazzo, Pastore, Wienold, \& Andersen, 2018; Chinazzo et al., 2019; Chinazzo, Wienold, \& Andersen, 2020; Chinazzo, Wienold, et al., 2018a; Chinazzo, Wienold, Andersen, \& Wienold, 2020). However, even if these effects have been reproduced through experiments, it is unclear whether they are noticeable under standard conditions in which the colour of the light and the appearance of spaces do not change that much (G. Chinazzo, personal communication, February 27, 2019). This motivates the introduction of a second assumption into this mathematical model: the effect of environmental cues on people's perceptions can be neglected without a significant loss of accuracy.

After these two assumptions, the need for incorporating Environmental Cues in the mathematical model is eliminated. Figure 11 shows the model of the Feeling of Comfort after introducing these two assumptions.

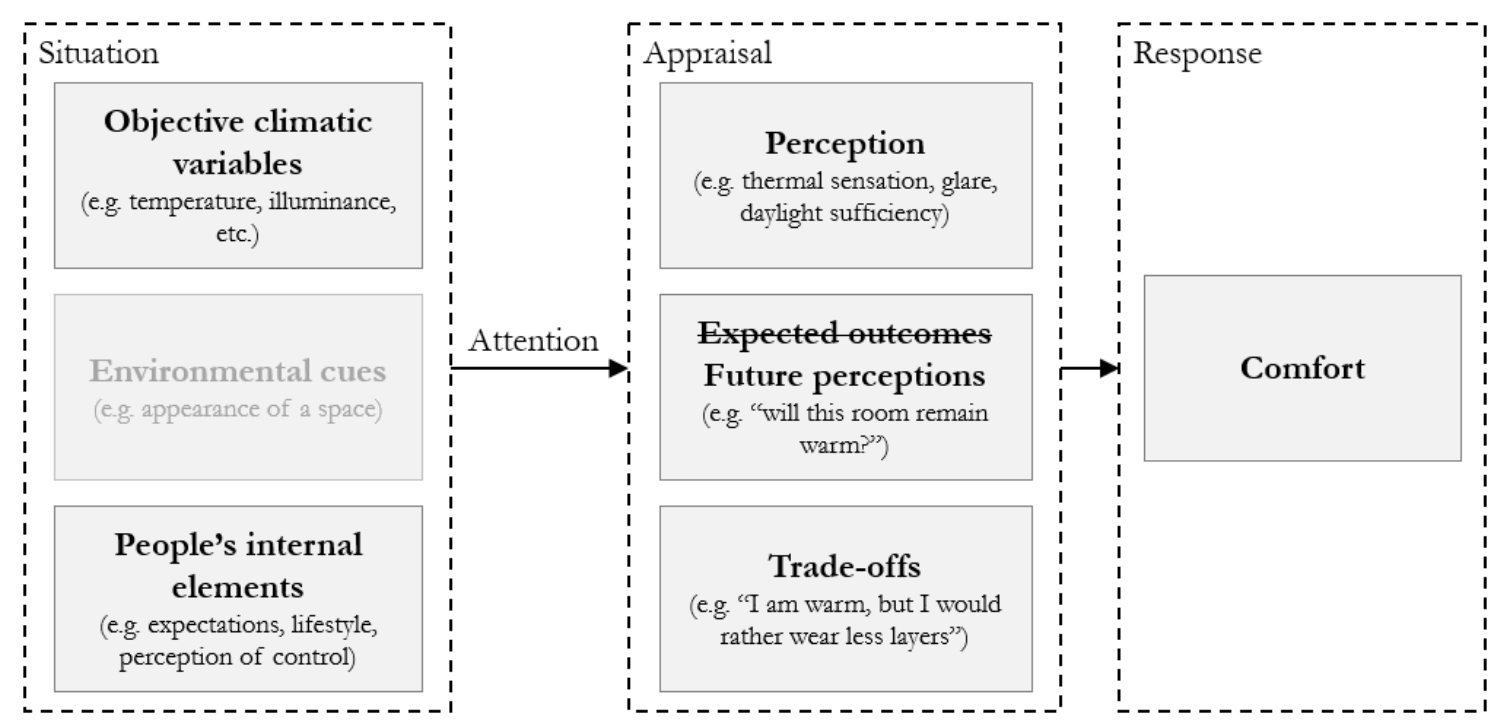

Figure 11: The Feeling of Comfort model adapted for its quantitative representation

What remains of this chapter will build - on top of these two assumptions-a set of mathematical equations that can be used to represent people's Feeling of Comfort in their homes. The development of this model utilizes a bottom-up approach. Specifically, it starts by defining a function that represents the "immediate satisfaction with a room" which can be used to derive the "immediate satisfaction with the dwelling". This last equation is used to derive a function representing the Feeling of Comfort that considers not only the here and now but the whole dwelling and also the future.

\subsection{Modelling the immediate satisfaction with the room}

The "immediate satisfaction with the room" is defined, within the context of this model, 
as the result of the cognitive process through which people's brains aggregate the meaning given to different aspects of a room into a single state of satisfaction. The factors that determine this satisfaction include all the elements that affect the present. That is to say, all the perceptions identified in chapters 5 and 6 as well as the influence of past choices over the present (i.e., the effect of trade-offs). The immediate satisfaction with the room is represented by the function $s(\vec{m})$, where $\vec{m}$ is the list containing the perceptions and the factors outside of the realm of building science that are relevant for people's wellbeing (i.e., those affected by Trade-offs, e.g., safety concerns). Through their behaviour, people will change the state of the dwelling and thus try to improve their immediate satisfaction.

The function $s(\vec{m})$ will be different for each individual, and thus it is this function that introduces most of the subjectivity to the model. The identification of this function requires careful consideration. A plausible way for doing so is to replicate the methods utilized by other comfort studies (Schweiker, Ampatzi, et al., 2020); that is, to correlate people's selfreported perceptions and satisfaction.

This approach was criticised in Section 2.3 because it gathered all individuals in the same group and thus ignored all their non-physical personal factors. However, chapters 5 and 6 identified a set of Internal Elements that explain part of the variability in people's satisfaction with the environment. This information can help to group individuals who-due to their non-physical personal factors-share a similar satisfaction function. This would lead to a set of more precise and customized equations representing the "immediate satisfaction with the room" for different individuals $(s(\vec{m}))$ that incorporate "the mind" as a factor that influences people's comfort. Unfortunately, this process will lead to functions that are representative of an average individual within a larger population (O’Brien et al., 2017), meaning that using it for designing homes does guarantee the satisfaction of every single person (e.g., ASHRAE, 1999; de Dear \& Brager, 2002). Despite this, it would be expected that the increased customization will lead to the satisfaction of a larger fraction of the population.

The elements in vector $\vec{m}$ can be estimated based on the set of quantifiable elements of the physical world $\vec{q}$ (note that environmental cues—often impossible to quantify-are assumed to be irrelevant). $\vec{q}$ not only contains variables that can be sensed by organs in the human body_-such as temperature and illuminance-but also other variables, such as the amount of clothing and the monetary cost of energy.

\subsubsection{Modelling perceptions}

As stated in Section 5.2.2, perceptions are how people interpret the stimuli received from 
the physical world and are often seen as the main drivers of comfort. Thus, perceptions can be directly considered as part of vector $\vec{m}$, and the elements that determine them can be directly incorporated into vector $\vec{q}$.

It is worth mentioning that chapters 5 and 6 proposed the existence of some new perceptions. For instance, the cleanliness of the space (for the domain of daylighting) and the perceptions of being a bother to others (in acoustics and air quality). Thus, incorporating these perceptions into vector $\vec{m}$ requires some research. Specifically, it requires the development of equations and models for estimating them as well as the determination of the relevant factors that should be incorporated into $\vec{q}$.

Table 17: Perceptions that affect the immediate satisfaction with a room

\begin{tabular}{ll}
\hline Perception & Domains \\
\hline Feeling of confinement & D, C, IAQ \\
Cleanliness of the space & D, C, IAQ \\
Daylight sufficiency & D, C, IAQ, W \\
Thermal sensation & W, C, IAQ \\
Odours & IAQ \\
Sensation when entering the space & W, C, D \\
Sense of connection with the exterior & D \\
Attractiveness of the space & D \\
Glare & D \\
Loudness & A \\
Bothering others & A , IAQ \\
A & A
\end{tabular}

\subsubsection{Modelling trade-offs}

Trade-offs reflect the fact that past choices affect the present by transferring nuisances from one domain to another one. As shown in Table 18, three different kinds of trade-offs should be modelled differently. Said table reveals that all of the trade-offs identified in chapters 5 and 6 can be included in one way or another within the calculation, although some 
of them require further research.

The first group includes those trade-offs that connect different domains of indoor environmental quality (e.g., daylight vs thermal comfort). These cross-domain trade-offs have the advantage of being implicit in the physical interaction of different domains of comfort. For instance, opening a window will impact the acoustic domain (e.g., some noise will come into the space), the indoor air quality domain (e.g., it will let air come into the building), the daylight domain (e.g., the amount of daylight transmitted through the façade changes) and so on. Thus, any tool capable of simulating all these effects simultaneously is already capable of accounting for the cross-domain trade-offs. The capability to estimate the relevant perceptions of each domain would have to be added if not readily available.

The second group includes all those trade-offs that connect indoor environmental quality domains with elements outside of common building science research. For instance, when improving thermal comfort results in increased utility bills (e.g., by turning a heater on) that might worry people. Contrary to the trade-offs in the first group, those in this one will have to be purposefully incorporated into assessment tools. For instance, the trade-off of utility bills can be incorporated into models as a perception depending on the expected monthly bill for the month. Likewise, the trade-off called an uncomfortable amount of clothing can be incorporated through a function that represents the pleasantness/unpleasantness of wearing a certain number of layers. Incorporating these trade-offs implies determining the functions that represent the Perceptions representing their corresponding level of annoyance.

The third group of trade-offs is a subgroup of the second group, including only those trade-offs that have very little impact on the everyday operation of dwellings. For instance, people do not need, on a daily basis, to choose between a more beautiful but colder floor and an, in comparison, uglier but warmer one. Likewise, after purchasing a dwelling, people are no longer capable of choosing between a quiet but remote location and a noisy but central one. The significance of this is that these trade-offs reflect choices that have already been made and that are unlikely to be unmade within the timeframe of the simulations. In other words, these trade-offs are special because they are not able to trigger behaviour. Furthermore, they are not able to suggest the possibility of behaviour, which means that they will not affect the "expected outcomes". So, by not reflecting any possible choice, these trade-offs can be considered to be unmodifiable parts of the environment. 
Table 18: How trade-offs can be incorporated into building performance simulation

\section{Trade-off}

Air quality vs acoustic performance

Thermal comfort vs acoustic performance

Thermal comfort vs air quality

Daylight vs thermal comfort

Coolness vs warmness

Light pollution

Complete silence vs sounds of nature

Loss of visual privacy

Utility bills

Uncomfortable amount of clothing

Energy efficiency

Exposure to wind

Bugs

Safety

Nuisances and lack of safety

Loss of views

Openness of the space

Sense of community vs acoustic performance

Location

Aesthetics

Increased maintenance and reduced durability

Damage to objects in the house

\section{Modelling method}

Already accounted for in simulations that consider both the air quality and the Acoustic domains.

Already accounted for in simulations that consider both the thermal and the acoustic domains.

Already accounted for in simulations that consider both the air quality and the thermal domains.

Already accounted for in simulations that consider both the daylight and the thermal domains.

Already accounted for in thermal simulations.

Already accounted for in lighting simulations.

Already accounted for in acoustic simulations.

Can be accounted for by explicitly including it in $\vec{m}$. Research needed.

Can be accounted for by explicitly including it in $\vec{m}$. Research needed.

Can be accounted for by explicitly including it in $\vec{m}$. Research needed.

Can be accounted for by explicitly including it in $\vec{m}$. Research needed.

Can be accounted for by explicitly including it in $\vec{m}$. Research needed.

Can be accounted for by explicitly including it in $\vec{m}$. Research needed.

Can be accounted for by explicitly including it in $\vec{m}$. Research needed.

Noise and indoor pollutants can be part of a multi-domain simulation. Since they are constant during the simulation, multiple scenarios can appropriately account for safety, ease of use, etc.

Can be included with regards to the operation of curtains and shading. Since they are constant during the simulation, multiple scenarios can appropriately account for the orientation and location of the dwellings.

Constant during simulation. Multiple scenarios can appropriately account for this. E.g., show performance and floorplans.

Constant during simulation. Multiple scenarios can appropriately account for this. E.g., show performance and characteristics of the neighbourhood.

Constant during simulation. Multiple scenarios can appropriately account for this. E.g., show performance and characteristics of the neighbourhood.

Constant during simulation. Multiple scenarios can appropriately account for this. E.g., show performance and renders of the dwelling.

Constant during simulation. Multiple scenarios can appropriately account for this. E.g., show performance, durability, and the need for maintenance.

Constant during simulation. Multiple scenarios can appropriately account for this. E.g., show performance and explain the potential damage to the objects in the dwelling. 
The fact that some trade-offs become part of the unmodifiable environment can help to reduce the complexity of the model because, very often, the best way of modelling the fixed characteristics of the environment is by not representing them at all within the math. On the contrary, these factors are often better represented through different media. For example, instead of developing the (probably, extremely complex) mathematical function representing the aesthetic value of a carpet, it is easier to simply simulate and compare two different scenarios. The first scenario will include a floor with the thermal properties of carpet; the second one, one with the thermal properties of timber. The simulation will provide data on how "warm" the two scenarios are, and the designer will then have to decide whether the extra aesthetic value of one-shown through pictures, videos, or other methods-is worth the extra nuisances caused by it. This kind of technique is commonly used today and thus this is not seen as a limitation of the model. Moreover, it might be the best way of combining the complexity of trade-offs and the Feeling of Comfort with the advantages of quantitative tools.

\subsection{Modelling the immediate satisfaction with the $d w e l l i n g$}

Section 7.2 discussed how to estimate the immediate satisfaction within a room considering the effect of trade-offs. This, however, only represents the satisfaction with the here and now and is not able to account for the whole dwelling. This limitation has to be addressed because respondents expressed an awareness of other areas of the dwellings, meaning that they perceive an immediate satisfaction with the dwelling as a whole as well. Specifically, they want to know that they can go to another room and still be comfortable. All rooms of an ideal home would be always comfortable.

"I would prefer to be able to, like, wear the same thing everywhere in my house and no need to like put on a jersey if I go to my bedroom or need a blanket on the couch because is cold, you know?"

Respondent 16 - Study 1, New Zealand

"I suppose that a warm house is one where, like, all the rooms in the house are warm. So, there is heat transfer between spaces"

Respondent 17 - Study 1, New Zealand

"[It means that] all spaces in general... wherever you go in the house you will have light, no matter how deep in the house you are"

Respondent 5 - Study 1, Chile 
"It is hard for me to think of a space that is not made better by having a window"

However, some responses also suggested that, if there was any need for prioritizing, that would likely be done based on the chances of going into different spaces.

"Living room and bedrooms are the most important [must have daylight]... Because it is there where you spend the most time... I do not care if the kitchen is naturally illuminated or not... You spend less time there, thus it is not a priority"

Respondent 7 - Study 1, Chile

"You generally are in the same places. And you need to take care of keeping those places warm"

Respondent 15 - Study 1, Chile

This suggests that the sense of immediate satisfaction with the dwelling as a wholerepresented by $\sigma$-can be modelled as the average immediate satisfaction of the different rooms or spaces, weighted by the importance of each of them at a certain time-represented by $p_{r}$ ( $r$ being the room number) — at a specific moment in time. This is shown in Equation 1.

$$
\sigma=\frac{\sum_{\text {rooms }} p_{r} \times s_{r}}{\sum_{\text {rooms }} p_{r}}
$$

It is important to notice that people not only care about their own comfort but that they also worry about other people's comfort. This implies that an individual's comfort can be determined by the conditions of the space he or she is in, as well as the conditions of the spaces where his or her family members are. This implies that the importance of different rooms is not mutually exclusive probabilities of visiting them (i.e., a person's "mind" can be in two rooms at the same time). Most of the variability of the factors $p_{r}$ will vary depending on the internal elements identified in chapters 5 and 6 , the most relevant probably being household composition (determining which rooms are relevant), and times of occupancy and lifestyle (determining when rooms are relevant).

\subsection{Modelling expected outcomes}

During the interviews, respondents expressed a constant awareness of the future. For instance, how a situation is appraised can vary depending on how long the pleasant or unpleasant elements in it are expected to last. Likewise, having or not access to mechanisms 
that can help to fix potential future nuisances might affect how comfortable a dwelling is perceived to be.

"We now have a construction in front of us. That has been noisier, but I know it is temporary. So, I do not [take it] too much into consideration"

Respondent 6 - Study 2, Chile

"In the summer [the train is] very noisy cause we have the bedroom window open and it is quite close. And it seems to have a lot of - it is a freight trainit seems to have a lot of carriages. So it goes for a long time."

Respondent 17 - Study 2, New Zealand

"Where you turn a heater on and it quickly heats everything up, in case you need it"

Respondent 15 - Study 1, New Zealand

"I prefer the cold [than the heat]. I like being able to go and warm myself up and snuggle up. I like the heat, actually, a lot as well... [I like the heat because it gives me] the ability to go swim in the river, I guess"

Respondent 18 - Study 2, New Zealand

"I prefer feeling cold [than hot]... I am not sure [why], I just suffer less. Also, you can always put on some more clothes when it is cold, but you cannot take all the clothes off",

Respondent 9 - Study 2, Chile

Consequently, people's Feeling of Comfort is affected by what they expect from the future. In other words, the "immediate satisfaction with the dwelling" $(\sigma)$ that individuals expect to experience a certain amount of time in the future will have an impact on their current Feeling of Comfort. (As stated in Section 7.1, what they expect can be approximated by what the future really will be). Mathematically, this can be represented as shown in equation 2, where $\kappa(t)$ is the Feeling of Comfort at time $t$, the function $\varphi$ is the impact of the future on people's current state of mind, and $\tau$ represents how much further into the future. As was done with $\sigma$, the value of $\kappa(t)$ is normalized (i.e., divided by $\left.\int_{0}^{\infty} \varphi(\tau) d \tau\right)$ so that its magnitude is comparable with those of $s$ and $\sigma$.

$$
\kappa(t)=\frac{\int_{0}^{\infty} \varphi(\tau) \sigma(t+\tau) d \tau}{\int_{0}^{\infty} \varphi(\tau) d \tau}
$$

But people are not likely to look too far into the future and not all future situations will be regarded as equally important. For instance, people are not expected to worry about how 
comfortable their home will be in seven days. They certainly care, however, about their comfort right now and, very likely, about how comfortable they will be in 30 more minutes. Based on this logic, it is expected that people consider the present to be more important than the near future, and the near future to be more important than the distant future. Thus, the impact of people's future satisfaction with their dwellings is expected to be the highest at the present and get lower with time. In other words, $\varphi$ is expected to be a monotonically decreasing function.

The function shown in Equation 3 fulfils this requirement and has the advantage of being flexible enough to represent different potential situations, including a quick or slow decay, the closer or further away in the future. Figure 12 shows how the value of $h_{1}$ can be varied to change the time at which the impact of the future starts to decay, and how $h_{2}$ represents the time after which the future has no impact on people's current Feeling of Comfort. The value of $\varepsilon$, on its part, is the value of $\varphi\left(h_{2}\right)$ and it should be small enough to be considered insignificant. The reason to include this parameter is that the function $\varphi$ never reaches 0 , and thus there is no real value for $h_{2}$ that will make the future irrelevant. Therefore, the value of $\varepsilon$ is needed to represent what the modeller means by the future being irrelevant $(\varepsilon=0.01$ was used for plotting Figure 12).

$$
\varphi\left(\tau, h_{1}, h_{2}\right)=\frac{1}{1+\left(\frac{1-\varepsilon}{\varepsilon}\right)^{\frac{2 \tau-\left(h_{2}+h_{1}\right)}{h_{2}-h_{1}}}}
$$

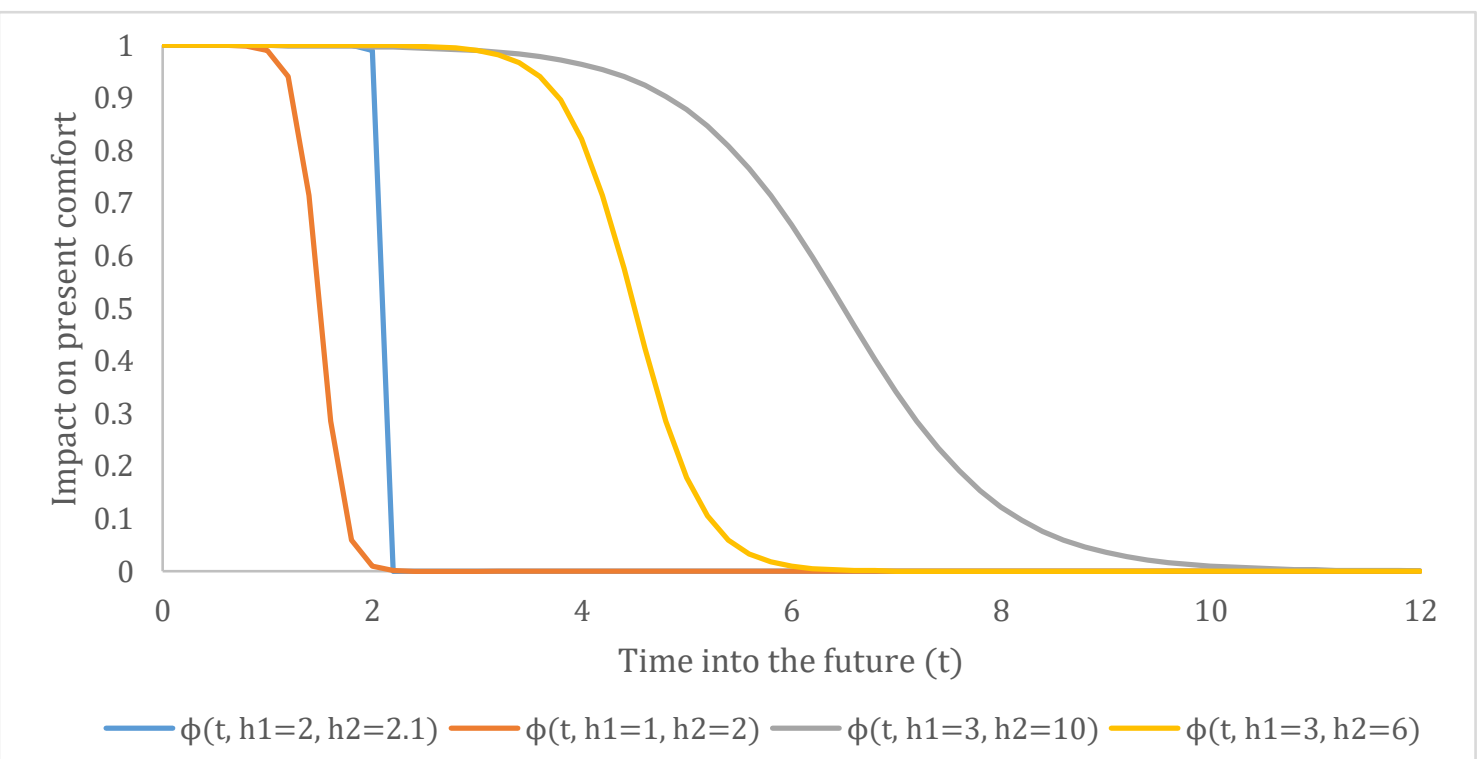

Note: This function is independent of the unit of time utilized. Meaning that as long as $h 1, h 2$ and $t$ are measured in the same units (e.g., days, hours, or seconds), this function remains valid.

Figure 12: Impact of future over the present Feeling of Comfort, according to Equation 3 
It is worth mentioning that the function $\kappa(t)$, introduced by equation 3 , acknowledges the effect of the future on people's present Feeling of Comfort, but it is limited to only one future. In real life, however, people have the option of choosing their future through the operation of the building (e.g., curtains and lights) and through adaptation (e.g., wearing more clothes). Moreover, systems — such as heaters or even windows and doors—can fail and thus they might be seen as unreliable. This implies the existence of several potential futures, all candidates to shape people's current Feeling of Comfort. There is even room for uncertainty, in which case more than one potential future might affect people's current state of the mind.

\section{"I like [to feel the rain] as long as I know it is not going to affect me"}

Respondent 12 - Study 1, New Zealand

"We had the hail the other night on the windows. And, I mean, it is a cool sound. But I do start to wonder whether or not my house can withstand it"

Respondent 15 - Study 2, New Zealand

Sections 7.4.1 and 7.4.2 explain how the systems' probability of failure (i.e., their reliability) and people's agency can be represented mathematically and introduced in the model.

\subsubsection{Incorporating systems' reliability}

Even if the first assumption introduced in Section 7.1 indicates that people tend to be relatively accurate at predicting what will happen, such a statement only applies to the physics of the building. This means that it does not include unpredictable events, such as the failure of a heating system or a window. However, even if people do not know when a system will fail, they have an idea of how likely it is that such a system will fail. In other words, people can infer the expected comfort. This can be mathematically represented as an expected value of $K(t)$, as shown in equation 4 . This equation considers the scenario in which the system fails $\left(\kappa_{f}\right)$, the perceived probability of it happening $\left(f_{p}\right)$, and the scenario in which the system does not fail $\left(\kappa_{n f}\right)$.

$$
\mathrm{K}(t)=f_{p} \times \kappa_{f}(t)+\left(1-f_{p}\right) \times \kappa_{n f}(t)
$$

It is worth mentioning that people's estimation of probabilities is often biased. For instance, people tend to overweight small probabilities and underweight moderate and large ones (Kahneman \& Tversky, 1979; Tversky \& Kahneman, 1992). Consequently, the value of $f_{p}$ will not necessarily be equal to the real probability of a system failing. For instance, people do not seem to expect any issue when switching the lights on, even if there must be a small 
but finite probability of failure. Similarly, the owner of a heater that fails two out of every three attempts to turn it on might perceive that the system always fails. Further research is required to determine whether the real probability of failure is a good approximation for the perceived one within the range of normal well-functioning systems.

It is also worth mentioning that evaluating $\mathrm{K}(t)$ does not require any stochastic component because knowing the probability of failure is enough. However marching forward through time within a simulation algorithm requires determining whether or not the system failed (using the real probability of failure, not the perceived one). Introducing this within a stochastic simulation is relatively simple.

\subsubsection{Incorporating people's agency}

Assuming that people know what will happen (and disregarding extraordinary events that may raise uncertainty), the effect of the expected outcomes over their current state of mind will depend exclusively on the expected comfort - $\mathrm{K}(t)$ —of one future scenario. And, since people will use their agency to maintain themselves as comfortable as they can, said scenario will be the most comfortable one they can achieve, identified as $\mathrm{K}_{b e s t}(t)$. For instance, when people see that sunset is coming-i.e., their homes will soon be dark and potentially cold_they will probably very quickly focus on that they can switch the lights and a heater on, and be reassured that they will still be comfortable?

Finding $\mathrm{K}_{\text {best }}(t)$ is very challenging because the number of possible futures to choose from increases non-linearly with the number of spaces in the dwelling, with the number of systems in each of them that can be operated, and with the opportunities for adaptations. As a result, the number of states that the system-i.e., the dwelling plus the occupant-can adopt will be unmanageably large for common situations (see Table 19 for some examples). Thus finding $\mathrm{K}_{\text {best }}(t)$ implies solving a highly complex mathematical problem on each simulation time-step.

\footnotetext{
7 The word "can" is emphasized because, even if people know they can turn heaters and lights on, this does not mean they will do it. This mathematical model represents cognition, not behaviour. However, if the occupants of a dwelling were to engage in action, such action would match the one represented by $\mathrm{K}_{\text {best }}(t)$.
} 
Table 19: Number of potential future scenarios for different dwelling/occupant settings

Dwelling/occupant being analysed

Number of potential future scenarios

One space with no systems to operate. No room for personal adaptation.

One space with no systems to operate. People can take clothes on and off.

One space with switchable lights. People can take clothes on and off.

Two spaces, each with switchable lights. People can take clothes on and off.

Two spaces, each with switchable lights and openable windows. People can take clothes on and off.

Three spaces, each with switchable lights and heaters, and openable curtains and windows. People can take clothes on and off.

Three spaces, each with switchable lights, ventilation and heaters, and openable curtains and windows. People can take clothes on and off.

Note: All states are presumed binary (e.g., windows are either open or closed) and manually operated.

Fortunately, people cannot be expected to solve this optimization problem during their everyday lives. On the contrary, people have bounded rationality (March, 1978; Simon, 1955; Thaler \& Sunstein, 2008), which means that they are more likely to use heuristics that help them achieve satisfying results than to pursue the best possible scenario. Consequently, incorporating these heuristics not only alleviates the computational intensity of the problem but is also a more realistic representation of people's thinking. Further research is required for understanding how exactly people select what they perceive to be the best possible future scenario (i.e., $\left.\mathrm{K}_{\text {best }}(t)\right)$. What remains of this section will present one example of an algorithm that helps to identify a handful of potential actions that should improve people's comfort. This algorithm first identifies the most worrying component affecting people's current Feeling of Comfort as well as the space where the issue is located. After this step, the number of future scenarios left to simulate is much more manageable and thus it is possible to estimate their outcome and choose the best one. The only restriction that this algorithm introduces is the assumption that $s(\vec{m})$ - people's immediate satisfaction with the room as a function of the relevant Perceptions that are part of $\vec{m}$-is linear in the parameters ${ }^{8}$. This means that that people's immediate satisfaction with a room at a certain time $t$ can be written

\footnotetext{
${ }^{8} \mathrm{It}$ is worth mentioning that this is not a great limitation because, according to Taylor's theorem, all smooth functions can be approximated by polynomials that have the shape of Equation 5. The biggest limitation of this assumption is that domains are assumed to be independent and thus cross-domain effects (e.g., the effect of Daylight over Thermal Sensation) are not accounted for.
} 
as shown in Equation 5.

$$
s(t)=\sum_{i} x_{i} m_{r i}
$$

Combining Equations 1 and 5 allows writing the immediate satisfaction with the dwelling as shown in Equation 6. Written in the form-as a double sum-the satisfaction with the dwelling can be conceptualized as the sum of all the elements of a table, in which rows represent the different rooms of the dwelling $(r)$; and the columns, the different $i$ elements of vector $\vec{m}$. In other words, the different Perceptions felt by the person in the dwelling.

$$
\sigma=\frac{\sum_{\text {rooms }} p_{r} \times \sum_{i} x_{i} m_{r i}}{\sum_{\text {rooms }} p_{r}}=\sum_{\text {rooms }} \sum_{i} \frac{p_{r} x_{i} m_{r i}}{\sum_{\text {rooms }} p_{r}}
$$

Additionally, combining Equations 6 and 2 allows deriving equation 7, which represents the Feeling of Comfort considering the effect of expected outcomes. Equation 7 is also a double sum, meaning that it can also be interpreted as the sum of the elements of a table in which columns are spaces or rooms, and rows are the Perceptions contained in vector $\vec{m}$. Equation 7 is the same as Equation 2, only constraining that people's immediate satisfaction with the room $s(\vec{m})$ conforms to Equation 5 (no assumption was made regarding the form of $s(\vec{m})$ before introducing Equation 5). This implies that Equations 2 and 7 hold the same meaning.

$$
\kappa(t)=\frac{\sum_{\text {rooms }} \sum_{i} \int_{0}^{\infty} \varphi \frac{p_{r} x_{i} m_{r i}}{\sum_{\text {rooms }} p_{r}} d \tau}{\int_{0}^{\infty} \varphi d \tau}
$$

After building this table, the cell with the lower value can be interpreted as the most worrying element affecting people's current Feeling of Comfort. The row and column of this element indicate which element of vector $\vec{m}$ should be improved and where this should take place. After identifying what to fix and where, it is time to choose a single action that will improve people's Feeling of Comfort. This requires understanding which actions have which effects. Fortunately, the data gathered in Studies 1 and 2 (Chapters 5 and 6) allowed identifying a series of actions that allow modifying the different elements of vector $\vec{m}$. Table 20 summarizes this information. Then, since the candidate actions associated with the elements in $\vec{m}$ are not very many, the number of potential futures is now reduced to a much more manageable amount. Consequently, the totality of these potential futures can now be simulated and, from the results, the one that produces the best Feeling of Comfort can be identified. 
As presented so far, the algorithm explained in this section only allows occupants to take a single action at a time. However, people often engage in multiple simultaneous actions (e.g., turn the lights off and open the curtains, opening windows and turning the heater off). This kind of behaviour can be emulated by recursively running the same heuristic several times, building on top of previous runs. For instance, the first run-i.e., the one that reflects the current real state of the dwelling - will indicate that closing the windows of the lounge area is a good idea. The second run-which already incorporates the fact that the windows in the lounge are closed-might then suggest that closing the windows in the bedroom is also a good idea. The third one — which already reflects the fact that all windows are closed—might suggest turning the heater on. The criteria for choosing the number of actions to take can be thought of in two complementary ways. First, it is possible that after some adaptation (e.g., after the windows are closed, and the heaters and lights are on) an extra action will not significantly affect the Feeling of Comfort (e.g., closing the curtains) in which case the occupant will not assess any more actions. The second possibility is to simply limit the number of actions the people can take, as they might not be expected to take too many. Choosing an appropriate threshold for this second criteria requires further research. 
Table 20: Strategies and actions that allow improving comfort

\begin{tabular}{|c|c|c|c|}
\hline Factor & Domains & Strategies to improve & Actions to improve \\
\hline $\begin{array}{l}\text { Feeling of } \\
\text { confinement }\end{array}$ & $\mathrm{D}, \mathrm{C}, \mathrm{IAQ}$ & $\begin{array}{l}\text { Ventilate, let daylight come } \\
\text { in }\end{array}$ & $\begin{array}{l}\text { Open windows, turn mechanical } \\
\text { ventilation on, open curtains }\end{array}$ \\
\hline $\begin{array}{l}\text { Cleanliness of the } \\
\text { space }\end{array}$ & $\mathrm{D}, \mathrm{C}, \mathrm{IAQ}$ & $\begin{array}{l}\text { Ventilate, let daylight come } \\
\text { in }\end{array}$ & $\begin{array}{l}\text { Open windows, turn mechanical } \\
\text { ventilation on, open curtains }\end{array}$ \\
\hline Daylight sufficiency & $\begin{array}{l}\mathrm{D}, \mathrm{C}, \mathrm{IAQ}, \\
\mathrm{W}\end{array}$ & Let daylight come in & Open curtains \\
\hline Thermal sensation & W, C, IAQ & $\begin{array}{l}\text { Warm up or cool down the } \\
\text { space, get more or fewer } \\
\text { clothes }\end{array}$ & $\begin{array}{l}\text { Open windows, turn HVAC } \\
\text { systems or heaters on or off. Add } \\
\text { or remove clothing. }\end{array}$ \\
\hline Odours & IAQ & Ventilate & $\begin{array}{l}\text { Open windows, turn mechanical } \\
\text { ventilation on }\end{array}$ \\
\hline $\begin{array}{l}\text { Sensation when } \\
\text { entering the space }\end{array}$ & W, C, D & $\begin{array}{l}\text { Not affected by building } \\
\text { operation }\end{array}$ & NA \\
\hline $\begin{array}{l}\text { Sense of connection } \\
\text { with the exterior }\end{array}$ & $\mathrm{D}$ & Let daylight come in & Open curtains \\
\hline $\begin{array}{l}\text { Attractiveness of the } \\
\text { space }\end{array}$ & $\mathrm{D}$ & Let daylight come in & Open curtains \\
\hline Glare & $\mathrm{D}$ & Block direct sunlight & Close curtains \\
\hline Loudness & A & Block exterior noise & Close windows \\
\hline Bothering others & A, IAQ & $\begin{array}{l}\text { Not affected by building } \\
\text { operation }\end{array}$ & NA \\
\hline Acoustic privacy & A & $\begin{array}{l}\text { Not affected by building } \\
\text { operation }\end{array}$ & NA \\
\hline Sound of silence & $\mathrm{A}$ & $\begin{array}{l}\text { Not affected by building } \\
\text { operation }\end{array}$ & NA \\
\hline Visual privacy & $\mathrm{D}, \mathrm{C}$ & $\begin{array}{l}\text { Reduce transparency of } \\
\text { façade }\end{array}$ & Close curtains \\
\hline Utility bills & W, C, D & Turn off appliances & $\begin{array}{l}\text { Switch lights and cooling and } \\
\text { heating systems off }\end{array}$ \\
\hline $\begin{array}{l}\text { Uncomfortable } \\
\text { amount of clothing }\end{array}$ & $\mathrm{W}, \mathrm{C}$ & Change clothing & Add or remove clothing \\
\hline Energy efficiency & W, C?, D? & Turn off appliances & $\begin{array}{l}\text { Switch lights and cooling and } \\
\text { heating systems off }\end{array}$ \\
\hline Exposure to wind & $\mathrm{C}$ & Seal the dwelling & Close windows and doors \\
\hline Bugs & $\mathrm{C}$ & Seal the dwelling & Close windows and doors \\
\hline Safety & A, C & Seal the dwelling & Close windows and doors \\
\hline Loss of views & $\mathrm{D}, \mathrm{A}, \mathrm{C}$ & $\begin{array}{l}\text { Increase transparency of the } \\
\text { façade }\end{array}$ & Open curtains \\
\hline
\end{tabular}

$\mathrm{A}=$ Acoustic, $\mathrm{D}=$ Daylight, $\mathrm{IAQ}=$ Air quality, $\mathrm{W}=$ Warmness, $\mathrm{C}=$ Coolness

A question mark next to a domain (e.g., X?) indicates that the code probably would be part of the domain, but was not identified in this particular dataset.

Fixed elements of the environment have been omitted. E.g., some materials and colours are perceived to be cleaner than others, but they are changed during the everyday operation of the dwelling. 


\subsection{Modelling attention}

As explained in Sections 5.2.2 and 6.2.2, the Feeling of Comfort only develops when a certain situation captures a person's attention. This is an important element in the model of the Feeling of Comfort because, if there is no attention, the situation will go unnoticed.

As explained in Sections 5.2.2 and 6.2.2, it would seem that people are less like to attend situations in which the indoor environmental conditions evoke a neutral set of perceptions (e.g., lack of nuisances) than others. However, relatively abrupt changes in the environmental conditions of the situation seem to be able to force attention, even if the change ultimately offers a more pleasant situation. Also, but not less important, arguably similar environmental conditions (or changes in them) are not equally likely to capture a person's attention. This might happen, for instance, because people's goals differ (e.g., reading a book vs enjoying a beer) and thus these situations also differ; and second, because people might pay attention to a situation due to psychological reasons. E.g., hearing an elderly person cough or seeing a child wearing no shoes might force attention to the temperature in the room.

For simplicity's sake, the model of attention introduced in this chapter will assume that the probability of a situation capturing a person's attention $(\alpha)$ is a function of how busy the person $(\beta)$ is and how satisfied is the individual with the space $(s)$. In other words-even if this can potentially be accommodated in a future version of the model—this model ignores the fact that abrupt changes in conditions change people's perceptions and most of the psychological reasons why people pay attention to situations. The reason to keep busyness $\beta$ as a factor is that it seems to have a relatively clear and simple relationship with attention.

\section{"When you are doing things in the house you do not notice [the noise]"}

Respondent 17 - Study 2, New Zealand

The rationale for using $S$ as opposed to the Feeling of Comfort $(\kappa$ or $\mathrm{K})$ is that, if the situation does not capture the individual's attention, no cognitive processes will be triggered and thus no inferences about the future will be built. With these insights, Equation 8 was proposed as a candidate for representing the probability of a person attending to a situation.

$$
\alpha(s, \beta)=\frac{1}{1+\left(\frac{1-\varepsilon}{\varepsilon}\right)^{\frac{2 s}{\beta}+1}}
$$

Equation 8 was designed by algebraically rearranging a Logit model, one of the most widely used models for representing probabilities of binary phenomena (Train, 2002, p. 38). 
The modification had the purpose of giving a specific meaning to the parameter $\beta$ : the subjective dissatisfaction at which it is nearly certain that the person will attend to the situation. In other words, evaluating Equation 8 with a value of $\beta$ equals to $-s$ leads to a probability of attention of nearly 1.0. The word nearly is emphasized because logit models never reach a probability of 1.0, and thus it is necessary to-as in Equation 3-introduce the parameter $\varepsilon$. This parameter represents a value that the modeller considers small enough so that $1-\varepsilon$ can be considered certainty; that is, nearly 1.0 (a value of $\varepsilon=0.01$ was used for plotting Figure 13, meaning that a probability of 0.99 was considered almost the same as a one of 1.0$)^{9}$.

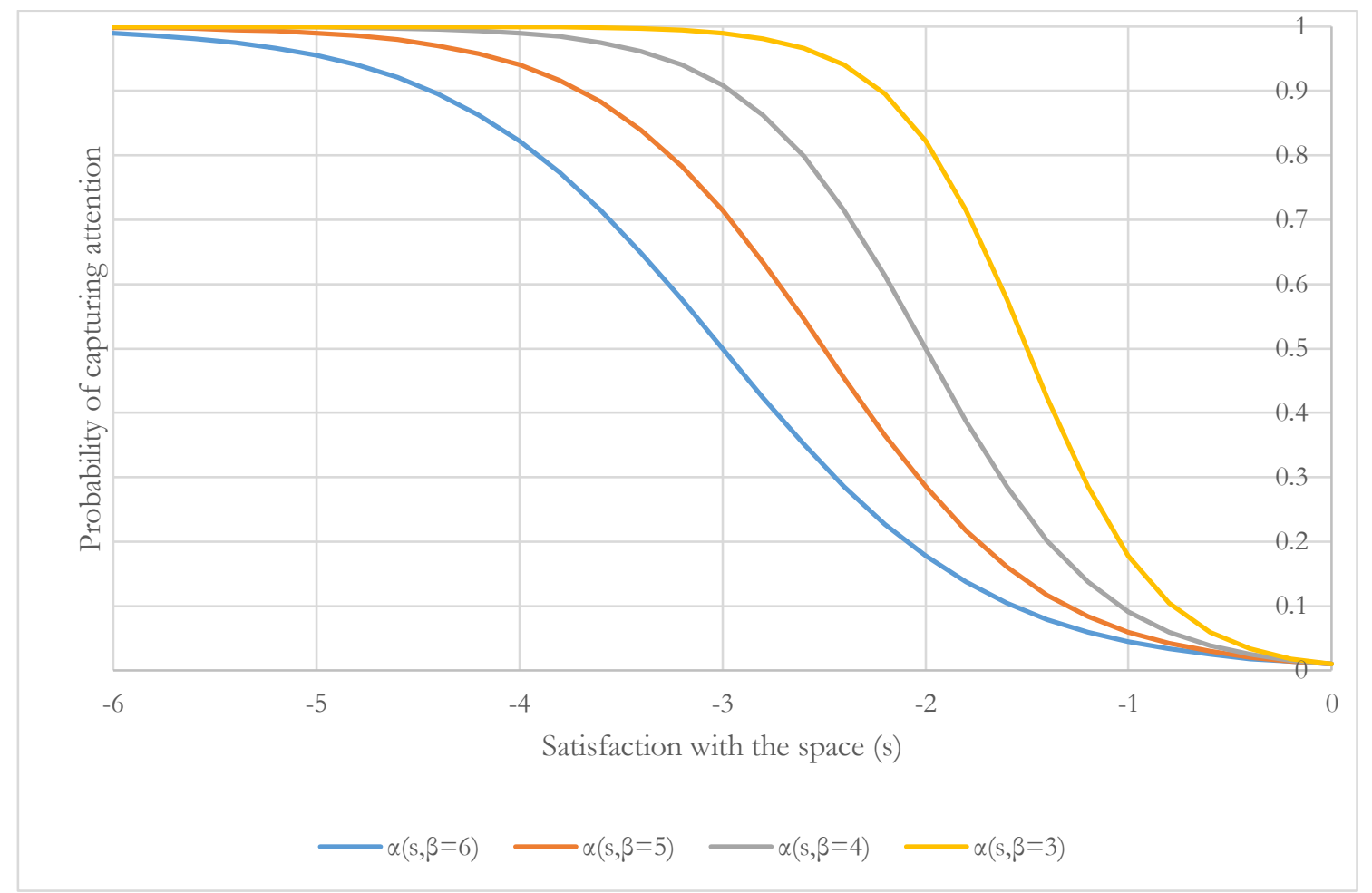

Figure 13: Probability of a person attending to a situation according to Equation 8

\subsection{Modelling behaviour}

Even if this dissertation is not about behaviour (but about cognition), both the qualitative and the quantitative models proposed by it have a big component related to people's actions. Thus, a simple function-Equation 9-is introduced to represent the probability of people engaging in adaptive behaviour. This function depends on the estimated increase in comfort that taking action would result in $(\Delta \mathrm{K})$ and on people's

\footnotetext{
${ }^{9}$ This kind of parameters are highly common in numerical methods. Among other things, they allow working with asymptotic functions (e.g., iterative algorithms, in which the error gets smaller and smaller, but never reaches zero) and comparing numbers that, due to their high precision, are very unlikely to be equal (e.g., is $\pi$ equivalent to 3.1415926 ?).
} 
Indifference $(\pi)$-i.e., an instrumental parameter representing how little a person is interested in fixing her or his discomfort. A value of $\pi=0$ implies that the individual will always behave if there is any benefit. As Equation 8, Equation 9 is designed as a logit model rearranged so that Indifference parameter $\pi$ had a specific meaning: the potential increase in comfort that virtually ensures that the person will engage in adaptive behaviour. In other words, evaluating Equation 9 with a value of $\Delta \mathrm{K}$ equal to $\pi$ leads to a probability of engaging in adaptive behaviour of nearly 1.0. Again, the word nearly is emphasized because logit models never reach a probability of 1.0 , hence the requirement for the parameter $\varepsilon$. This parameter has the same meaning in Equation 9 as that given in Equation 8, explained earlier in this section.

$$
\mathrm{B}(\Delta \mathrm{K}, \pi)=\frac{1}{1+\left(\frac{1-\varepsilon}{\varepsilon}\right)^{\frac{-2 \Delta \mathrm{K}}{\pi}+1}}
$$

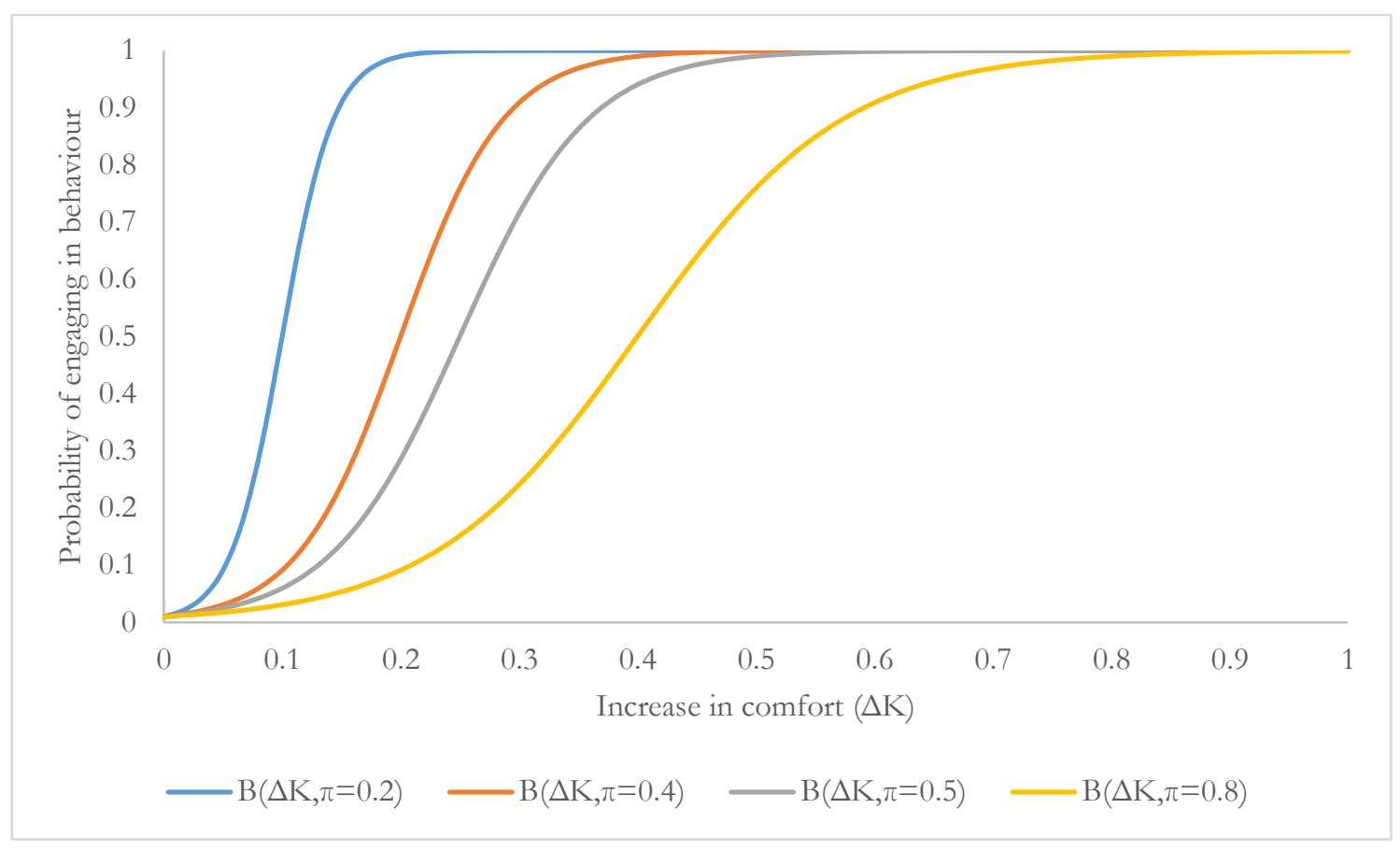

Figure 14: Probability of engaging in a behaviour, according to Equation 9

\subsection{Conclusions}

This chapter discussed how the Feeling of Comfort can be modelled mathematically and introduced a set of equations that could do so. A bottom-up approach was utilized. This means that that the Feeling of Comfort_-including the effect of people's awareness of the future-was composed by starting from a model of the immediate satisfaction with the here and now followed by a model of the immediate satisfaction with the dwelling, and then extended by incorporating the impact of the expected outcomes and people's agency. 
The applicability of this model is limited in two ways. First, it assumes that the expected outcomes that people infer are a reasonably good prediction of the future and thus they can be approximated by the estimations of simulations. This is likely to be a good assumption when modelling the Feeling of Comfort in a highly familiar environment (e.g., people's homes), but the error it can introduce remains to be tested. The second limitation is that the effect of Environmental Cues (Tables 4 and 5) on people's perceptions has been disregarded. This effect is known to exist, but it has only been observed in laboratory experiments representing relatively extreme conditions that might not resemble real architectural settings.

Apart from these limitations, it is important to notice that the mathematical model presented here has not been tested and validated. In fact, due to the objective of this research-to explore "the mind" in the context of people's comfort and propose ways in which it can be better incorporated into building science's research and practice-this mathematical model was never meant to be finished. On the contrary, the purpose of this chapter is to demonstrate how the Feeling of Comfort model can inform building science's research and practice. This demonstration was offered through an example: the development of a mathematical model of comfort and (potentially) behaviour.

Since the purpose of this chapter is not to produce a fully finished mathematical model of comfort but to provide examples of how the Feeling of Comfort model can be used to inform building science research and practice, the equations presented in this chapter should not be seen as definitive. That is to say, there is room for improving and even replacing all the equations that make up the mathematical model. Nevertheless, this chapter has demonstrated that it is possible to incorporate more of "the mind" into building science's comfort research by using the insights offered by the qualitative Feeling of Comfort model. These insights can be used with different objectives as well. For example, artificial intelligence and machine learning can be used instead of the equations presented in this chapter (e.g., Halhoul Merabet et al., 2021), as done when implementing personal comfort models (Joyce Kim, Schiavon, \& Brager, 2018). This kind of development can also use the insights that the qualitative Feeling of Comfort model can offer. For example, the concept of Trade-Offs suggests that people's behaviour does not always lead to a subjectively comfortable indoor environmental quality. This means that the data gathered through sensors or observations should be interpreted carefully. This insight is crucial for implementing artificial intelligence and machine learning approaches, which require an existing dataset to be developed (Fan et al., 2021). Similarly, the Internal Elements of the Feeling of Comfort models can help better categorize and classify the data used to train artificial intelligence modules. For example, the data in Table 5 suggests that the expected behaviour of people is likely to depend on their 
budget and their household composition. This information can be useful for deciding how many models should be developed (e.g., one for young people with no children, one for young people with children, and one for older people). Finally, an important deficiency of this chapter is that, despite offering a set of equations that can help represent the Feeling of Comfort in quantitative terms, it lacks a solution or implementation of these equations. Specifically, these equations are not part of any building performance simulation tools, which is a core element of the toolset utilized in building science research and practice. Therefore, the following chapter discusses the implementation of these equations in building performance simulation tools and also develops a prototype of said implementation. 


\section{TOWARDS A TOOL FOR SIMULATING THE FEELING OF COMFORT}

"Talk is cheap. Show me the code"

LINUS TORVALDS

The previous chapter discussed how the model of the Feeling of Comfort introduced in Chapter 5 can be represented in mathematical terms through a set of equations. However, due to their novelty, these equations are not currently part of any building performance simulation tool. Since these tools play a fundamental role in building science research and practice, equations alone (i.e., without any method for solving them) might be considered to be only a partial answer to Research Question 3 introduced in Section 3.2-i.e., how can predictive models of comfort incorporate people's non-physical characteristics and their cognitive processes?. This chapter addresses this research question. Specifically, it complements Chapter 7 by implementing the equations it introduced into a prototype building simulation tool. Some results produced by this prototype are also shown. Together, these two outcomes-results from simulation and the design of the building performance simulation tool—are part of the response to Research Question 3.

Enabling existing building performance simulation tools to utilize the equations of Chapter 7, however, is unlikely to be an incremental update but a significantly disruptive one (Section 8.1 discusses this in more detail). Because of this, this chapter not only discusses the algorithms required to solve the equations introduced in Chapter 7 but also the requirements and design considerations of any software that intends to implement these algorithms.

It is worth mentioning that the design of the simulation tool introduced in this chapter was produced through an iterative process of testing and prototyping. Therefore, the algorithms, the design, and the source code shown in this chapter do not presume to be the best. On the contrary, they simply present an answer to the question of how can predictive models of comfort incorporate people's non-physical characteristics and their cognitive processes? The purpose of this tool-and this chapter-is to show that the Feeling of 
Comfort model can be incorporated into simulation tools and thus that it could become a useful and practical framework for evaluating building designs.

\subsection{Requirements for the simulation tool}

Building performance simulation tools are different to each other in many ways. For example, some tools are developed for commercial use (Guglielmetti, Macumber, \& Long, 2011; Jakubiec \& Reinhart, 2011) while others were designed with a more experimental purpose in mind and thus they are constrained to specific use cases (Bustamante, Uribe, Vera, \& Molina, 2017; Janak, 1997; Vera, Bustamante, Molina, \& Uribe, 2016). Likewise, it is possible to classify simulation tools based on the domains they can simulate. For example, some tools are only prepared to simulate a single domain, such as daylight (Molina, 2018; Reinhart, 2013; Ward, 1994; Ward \& Shakespeare, 1998); others, mostly focus on one domain and treat another one as secondary (Crawley et al., 2001, 2004); and others, try to emphasize multiple domains equally (Jakubiec \& Reinhart, 2011). Furthermore, building performance simulation tools that are specialized in the same domains also differ from each other. These differences can be noticed in the methods they use and the approaches they take to perform simulation (Ayoub, 2019, 2020; Crawley et al., 2008; Ochoa, Aries, \& Hensen, 2012). It is, therefore, necessary to discuss the requirements that a simulation tool whose purpose is to simulate the Feeling of Comfort needs to comply with. This section examines these requirements.

\subsubsection{It must be able to handle multiple domains simultaneously at runtime}

As explained in Section 5.2.3, one of the three elements that determine the Feeling of Comfort is trade-offs. That is to say, the sacrifices people often need to make on one aspect of their lives to improve another. This suggests that, as opposed to scientists, people do not strictly separate the thermal, lighting, acoustic and air quality domains. Therefore, any software or simulation algorithm that attempts to incorporate the Feeling of Comfort must be able to handle multiple domains simultaneously.

Handling multiple domains simultaneously is not a straightforward task. Part of the reason for this is that there are significant differences in the physical phenomena, the calculation methods, and the mathematical models involved in these different domains (Molina, 2014). Despite this, several examples of these integrated simulations are available in the literature. For instance, it is possible to perform integrated lighting/thermal simulations by running a lighting simulation whose results then serve as inputs to a thermal simulation (Bustamante et al., 2017; Guglielmetti et al., 2011; Jakubiec \& Reinhart, 2011; Molina, Vera, 
\& Bustamante, 2014; Vera et al., 2016; Wienold, Frontini, Herkel, \& Mende, 2011). A limitation of this approach, however, is that the second simulation does not have the chance of influencing the first one. In other words, this approach presumes that all the adaptive behaviour (e.g., opening and closing curtains and windows, switching the lights on and off) is triggered by the domain handled by the first simulation (Vera et al., 2016). This is not acceptable for simulating the Feeling of Comfort.

Overcoming this limitation implies having bi-directional communication between tools. This implies performing calculations on every domain at each time step of the simulation (as opposed to running one after the other), treating the combination of domains as a single physical system. It is possible to do this by combining already existing tools, as shown by Janak (1997), who combined ESP-r (Energy Systems Research Unit, 2021) and Radiance (Ward, 1994) which focus on the thermal and lighting domains, respectively. This practice, however, is rarely straightforward because most of the existing simulation tools have not been designed to interact with each other. For instance, Radiance's source code states that “these [Radiance's] routines are designed to aid the programmer who wishes to call Radiance as a library. Unfortunately, the system was not originally intended to be run this way, and there are some awkward limitations to contend with" (NREL, 2019). The alternative is to develop specific calculation routines and functions that are, from the very beginning, meant to be integrated. This is what Tzempelikos and Athienitis (2007) and Petersend and Svendsen (2010) did in their studies. The tools described by these authors do not seem to be publicly available.

Simulating the Feeling of Comfort implies performing a building simulation thatsimultaneously and at runtime-considers multiple domains. However, the existence of algorithms that handle two domains (e.g., the examples shown in this section) suggest that it is possible to extend these algorithms to multiple domains.

\subsubsection{It must allow travelling back in time}

As explained in Section 5.2.3, the Feeling of Comfort seems also to depend on Expected Outcomes. In other words, it depends on what people think will happen in the future. As argued in Section 7.1, these inferences can be approximated by simulation results if the environment being simulated is highly familiar to the person whose Feeling of Comfort is being estimated. This approximation should hold when simulating people's Feeling of Comfort in their own homes.

Even if it sounds simple, approximating Expected Outcomes by simulation results 
presents a challenge. Specifically, it requires a simulation tool that, at every time step, can advance a few hours into the future (e.g., emulating people's inferences) and then come back to the present as if nothing happened. As far as the author of this dissertation is aware, no building simulation tool is currently able to do this. Furthermore, providing existing building simulation tools of such a capability probably implies performing drastically invasive changes to the way they operate. Therefore, developing a new tool—as undertaken by Tzempelikos and Athienitis (2007) and Petersend and Svendsen (2010)_might be an easier and more effective option.

\subsection{An architecture for simulating the Feeling of Comfort}

As explained in Section 8.1.1, performing multi-domain building simulation is not a simple task. Part of the reason for this is that simulation tools focused on one domain are different from those in other domains because they use different mathematical representations of building elements (e.g., walls and windows), focus on different physical phenomena, and utilize different mathematical methods for solving their problems. Due to these differences, brute-forcing these domains into a single software package (e.g., by copying and pasting parts of the source code of already existing tools, or simply putting together code associated with different domains into a single source-code file) will likely produce an unmaintainable tool. That is to say, the resulting code would have elements associated with different domains scattered all over its files, meaning that researchers and practitioners - whose expertise is often limited to one or two of these domains-would hardly be able to understand it or extend it. As shown in Figure 15, the tool described in this chapter avoids this problem by having a modular architecture that respects the separation of domains that has traditionally existed in building science.

Even if it makes the software easier to maintain, a modular architecture does not address the challenge of integrating the different domains (it, indeed, keeps them separated). The tool described in this chapter addresses this issue through two characteristics. First, this tool requires that all the modules embedded in it adhere to certain communication protocols and functionality. This is sometimes called interface-based architecture (Klabnik \& Nichols, 2021) and can be enforced at compile time. And second, it asks its modules to communicate with each other by reading and writing from and into a single, relatively small, shared data structure- the Simulation State. This enables the different modules to communicate with each other straightforwardly and also to simulate multiple potential outcomes in a somewhat easy and efficient manner. Since it seems to be a new concept in building performance simulation, this second characteristic is explained in more detail in the following section. 


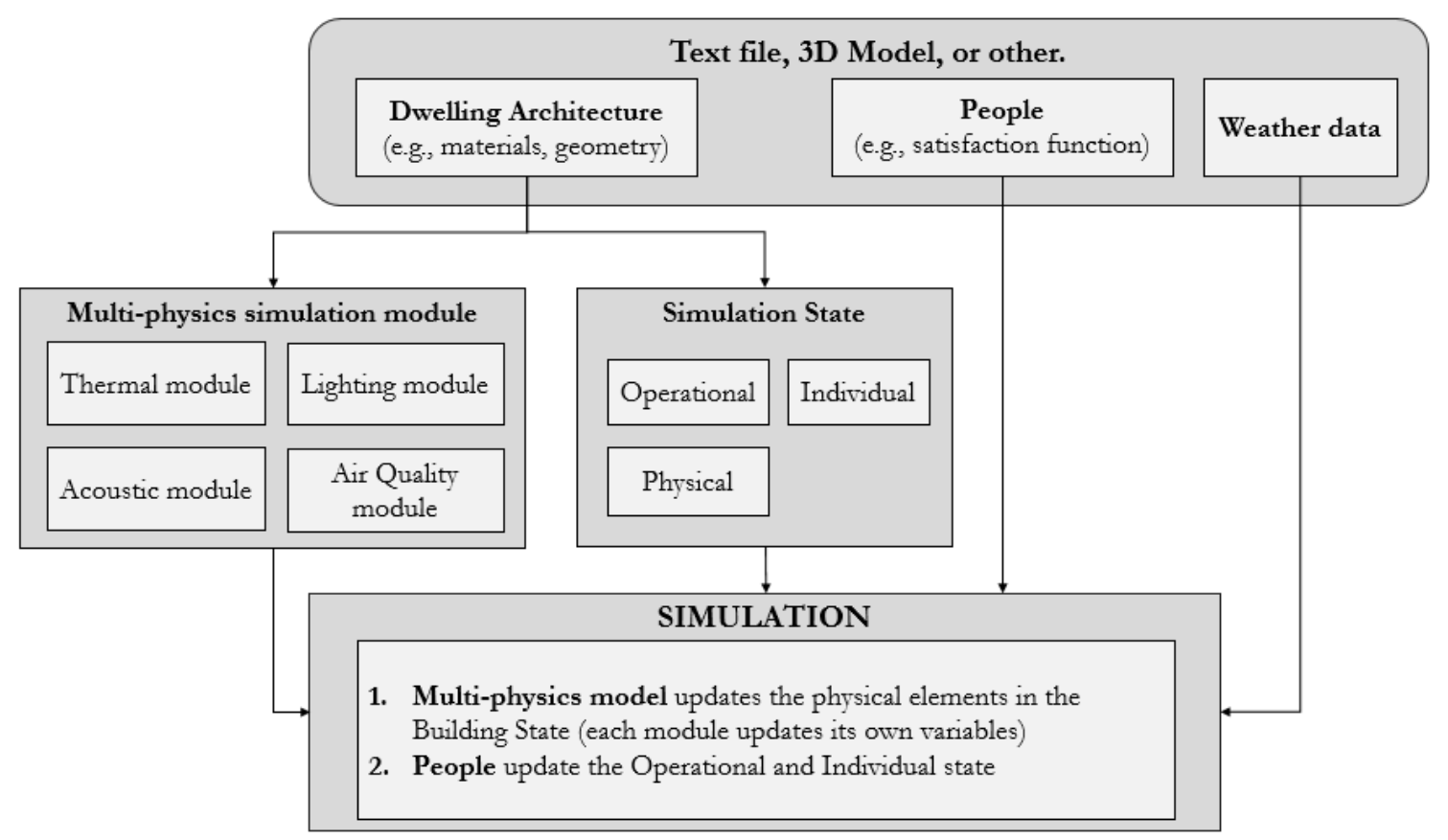

Figure 15: Architecture of tool for simulating the Feeling of Comfort

\subsubsection{Benefits of sharing data through a single and relatively small data structure}

While researchers and practitioners are not expected to have extensive knowledge about all the different domains affecting comfort, they are certainly expected to understand which factors associated with other domains affect the one they are specialized in. For instance, a person capable of developing the thermal simulation module will not necessarily know about lighting simulation; however, such a person will probably know if-and how-the solar radiation over the walls affect the heat transfer in the building. This is the concept that the simulation state-i.e., the shared and relatively small data structure that enables the communication between modules-intends to reflect. This allows a person working on the thermal model to ask for data associated with other modules-e.g., about solar radiationwithout necessarily knowing how such a piece of data was calculated or even how the module that calculated it works.

Operationally, this works as follows: at every time step in the simulation, each module will read some data from the simulation state, perform some calculations, and then write the updated data back into the simulation state. Other modules can then do the same, thus successfully exchanging data at runtime.

Since they focus on the physical phenomena, the thermal, acoustic, lighting and air quality modules will mainly read and write physical elements on the simulation state (e.g., temperature, illuminance). Additionally, these modules also read operational elements, such as the state of the windows and luminaires (e.g., Open/Close, On/Off) and others. People, 
on the other hand, will read from the physical elements (e.g., sensing Temperature) and will write operational and individual (e.g., amount of clothing) elements. Figure 16 graphically shows this.

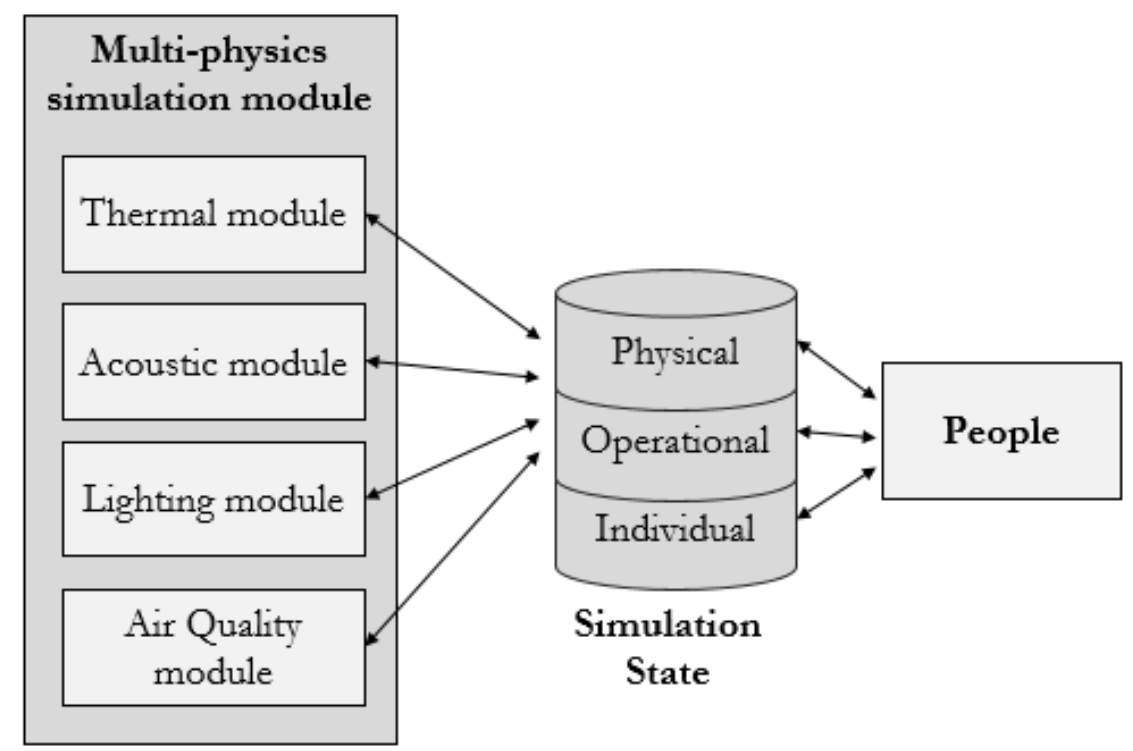

Figure 16: Paths of communication between the different modules

Apart from enabling communication between modules, the simulation state allows for a relatively efficient and clean simulation of potential outcomes. This is possible because of two reasons. First, since the simulation state is rather small in size, it can be quickly copied, cloned and deleted. Second, because it contains all the data required for the simulation modules to perform their calculations, this structure allows the simulation program to keep an untouched copy of the present while simulating various potential futures. Specifically, as shown in Figure 17, this tool relies on disposable copies of the simulation state that represent different potential outcomes. This implies that discarding these outcomes (e.g., because a person decides that the benefit in comfort is not worth the effort or because none of them improves the person's comfort) is just a matter of deleting these new structures and plugging the original one into the simulation. If, on the contrary, one of these outcomes is chosen as the way to go, then such a simulation state can replace the original one and the simulation continues normally. 


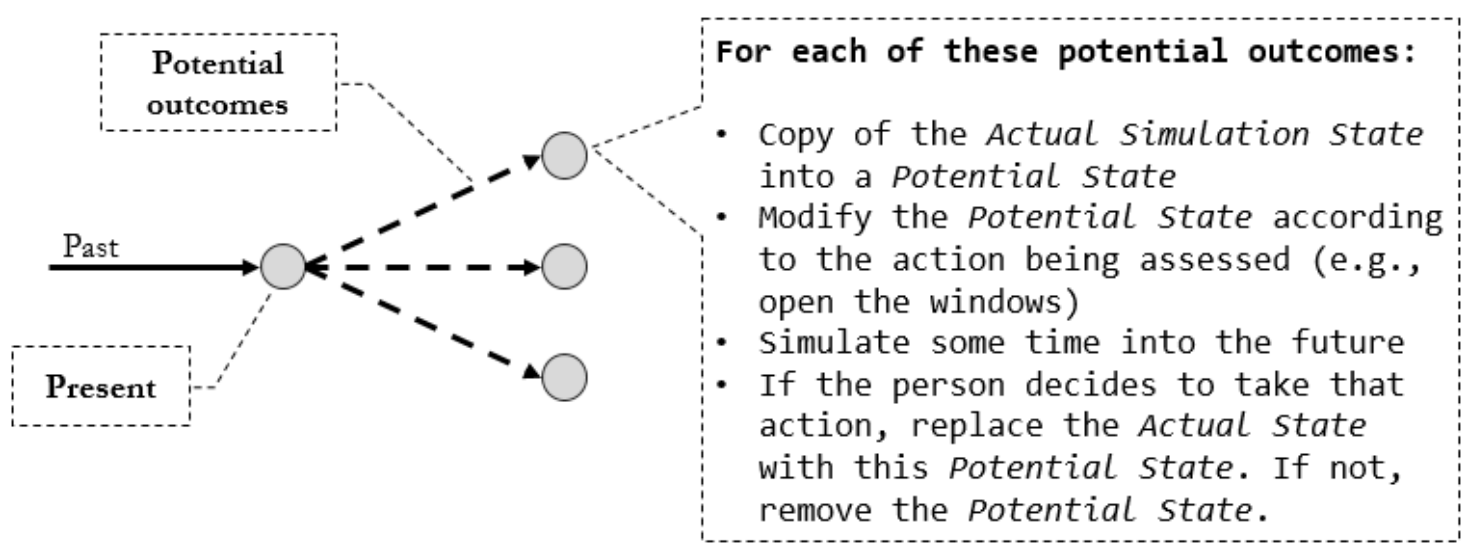

Figure 17: Algorithm that allows for an efficient simulation of potential outcomes

\subsection{Simulating people's Feeling of Comfort and behaviour}

The previous section described a software architecture that allows the simulation of the Feeling of Comfort. In other words, a software architecture that complies with two requirements: it enables the communication between the different simulation domains-e.g., thermal, acoustic, lighting and air quality-at runtime, and allows simulating expected outcomes in a relatively efficient and simple manner. Additionally, Chapter 7 explained how the Feeling of Comfort can be represented mathematically. This section introduces an algorithm that represents, in a simplified manner, the development of the Feeling of Comfort. Figure 18 shows this algorithm, emphasizing how it matches with the model of the Feeling of Comfort as shown in Figure 11.

As explained in Section 7.5, the development of the Feeling of Comfort starts after a person has paid attention to the current situation. Said section also argued that the probability of attending a certain situation-even if further research is required to understand this better-can be modelled as a function of the current satisfaction with the space $(s$, see Equation 8). However, estimating this probability in a simulation environment implies knowing where the person is at each moment in time. While this is potentially possible, the tool introduced in this chapter simplifies this by making the probability of attending a function of the immediate satisfaction with the dwelling ( $\sigma$, see Equation 1$)$. This satisfaction is calculated based on the current Simulation State (e.g., clothing, temperature, illuminance, etc.) as well as the person's satisfaction function (see Equation 5). 


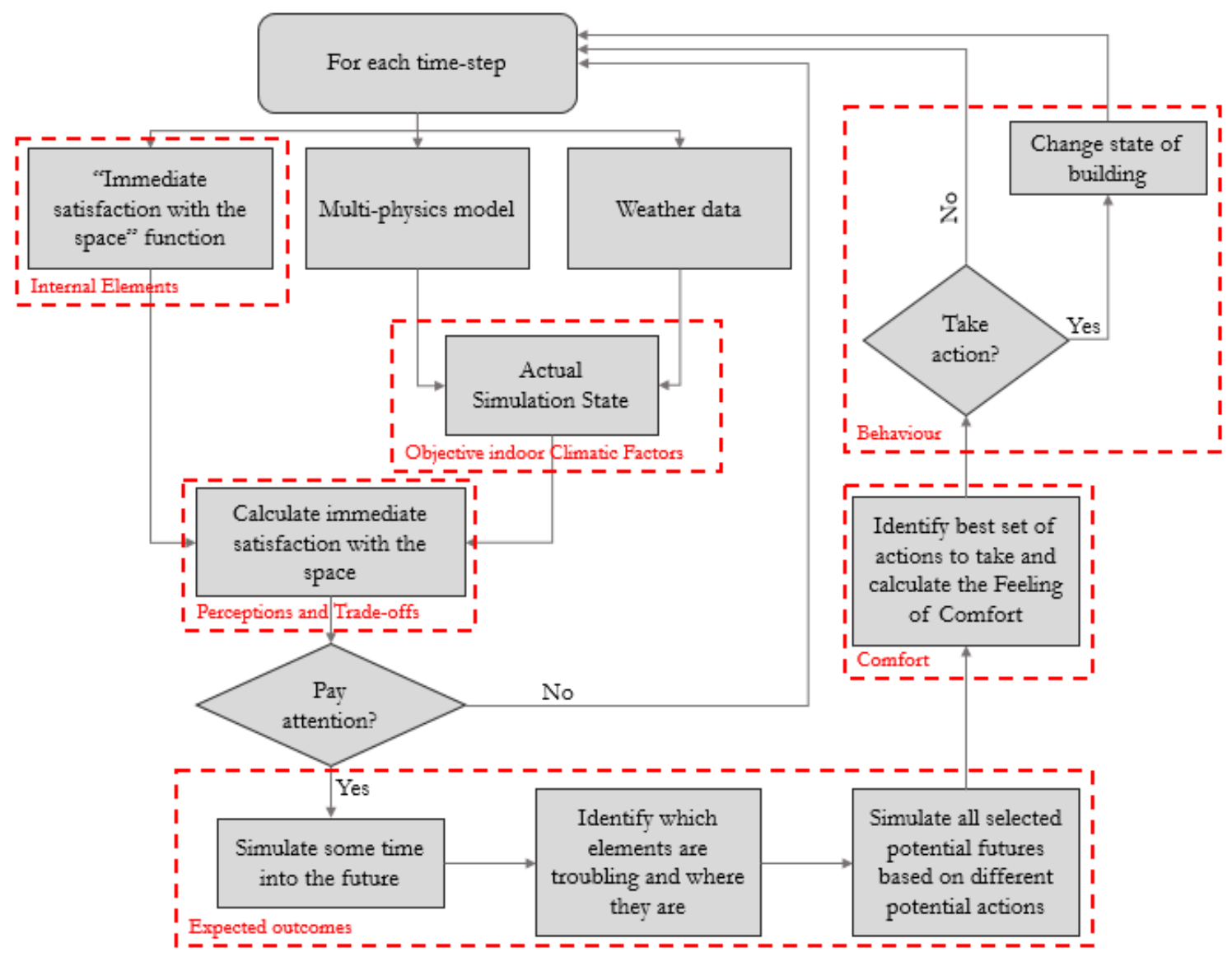

Figure 18: Algorithm used for incorporating the Feeling of Comfort in building simulation

If the person attends the situation, then she or he will foresee the future, identifying which elements should be fixed and where they are in the dwelling (see Equation 7). From this, a set of potential actions can be identified based on common heuristics (e.g., see Table 20). Each of these strategies leads to a different potential outcome, and thus all these actions are simulated according to the algorithm outlined in Figure 17. The comfort associated with the best of these potential outcomes is considered to be the Feeling of Comfort $\left(\mathrm{K}_{\text {best }}\right.$, see Section 7.4.2). As explained in Section 7.4.2, a recursive implementation of this process allows the simulated person to take multiple actions simultaneously (e.g., opening the windows and turning the lights off, both at the same time).

After developing a certain Feeling of Comfort, people will decide whether they will take action or not. This stage is modelled according to Equation 9 (see Section 7.6).

\subsection{Example of a simulation}

Having described the design and architecture of a building performance simulation tool capable of modelling people's Feeling of Comfort, this section will show results produced by a prototype version of said implementations. The purpose of this is twofold. On the one hand, it intends to show that simulations can potentially incorporate the Feeling of Comfort; 
and on the other hand, to explore the implications of the Feeling of Comfort further.

It is worth mentioning, however, that the focus of this prototype tool lies exclusively on the Feeling of Comfort and not on physics. As such, the lighting, thermal and acoustic simulation modules are only mock-ups that emulate physical behaviour and have not been validated. Furthermore, no air quality module has been implemented. Developing better simulation modules is considered future research.

\subsubsection{Inputs to the simulation}

As explained in Section 8.2 (Figure 15), the main inputs to the simulation are the dwelling's Architecture (i.e., geometry, materials, etc.), the People (e.g., their satisfaction function, proactivity, busyness, etc.) and the Weather data. An overview of the code that defined the inputs can be found in Appendix V.

The weather data utilized for the simulations was the Typical Meteorological Year corresponding to Santiago (Chile), acquired from the EnergyPlus website (U.S. Department of Energy, 2021). Since the information about external noise is absent from Typical Meteorological Years—but required by the acoustic model—a daily schedule was provided. This schedule is meant to represent a commuter road noise scenario during a weekday. It is shown in Figure 19. While the translation of external noise into internal Loudness was performed through a mock-up simulation module, this can potentially be improved by using measured traffic noise data and a tool for predicting sound insulation through buildings' facades (INSUL, 2021).

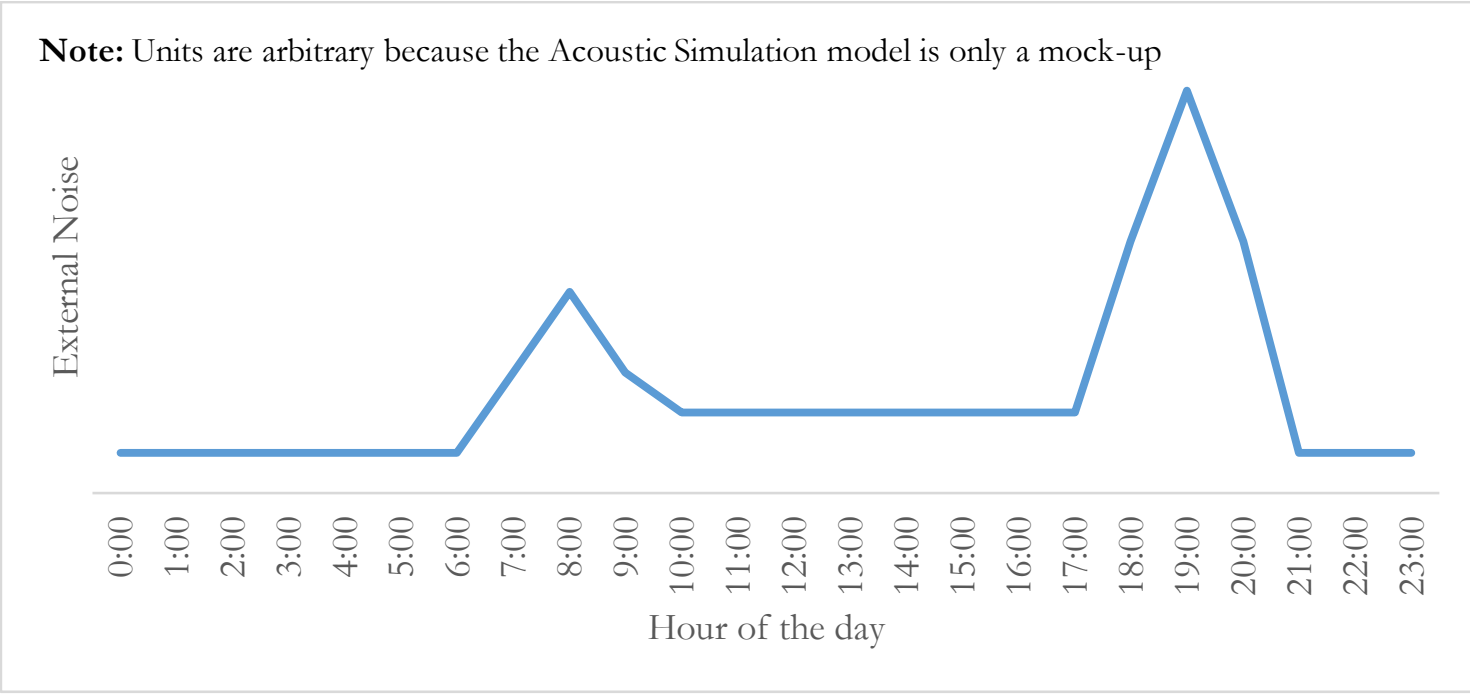

Figure 19: External noise level utilized during the simulation

As per the dwelling's architecture, it is shown in Figure 20. It is a two-bedroom dwelling with a separate Kitchen and Bathroom, and a Hallway that serves as a connection between 
them. From these six spaces, the most important ones for the people who live in this dwelling are the Bedrooms and the Living room. It is worth noting that, while the importance can potentially vary over time, the simulation assumes it to be constant. Table 21 shows the relative importance of the different spaces. Since the focus of this simulation is on people's Feeling of Comfort and thus the physical components of the simulation are only mock-ups, the details of the physical properties of the dwelling are not considered relevant.

Table 21: Relative importance of the different spaces

\begin{tabular}{lr}
\hline Space & Relative importance \\
\hline Bedroom 1 & 1.0 \\
Bedroom 2 & 1.0 \\
Living room & 1.0 \\
Kitchen & 0.1 \\
Bathroom & 0.03 \\
Hallway & 0.01 \\
\hline
\end{tabular}

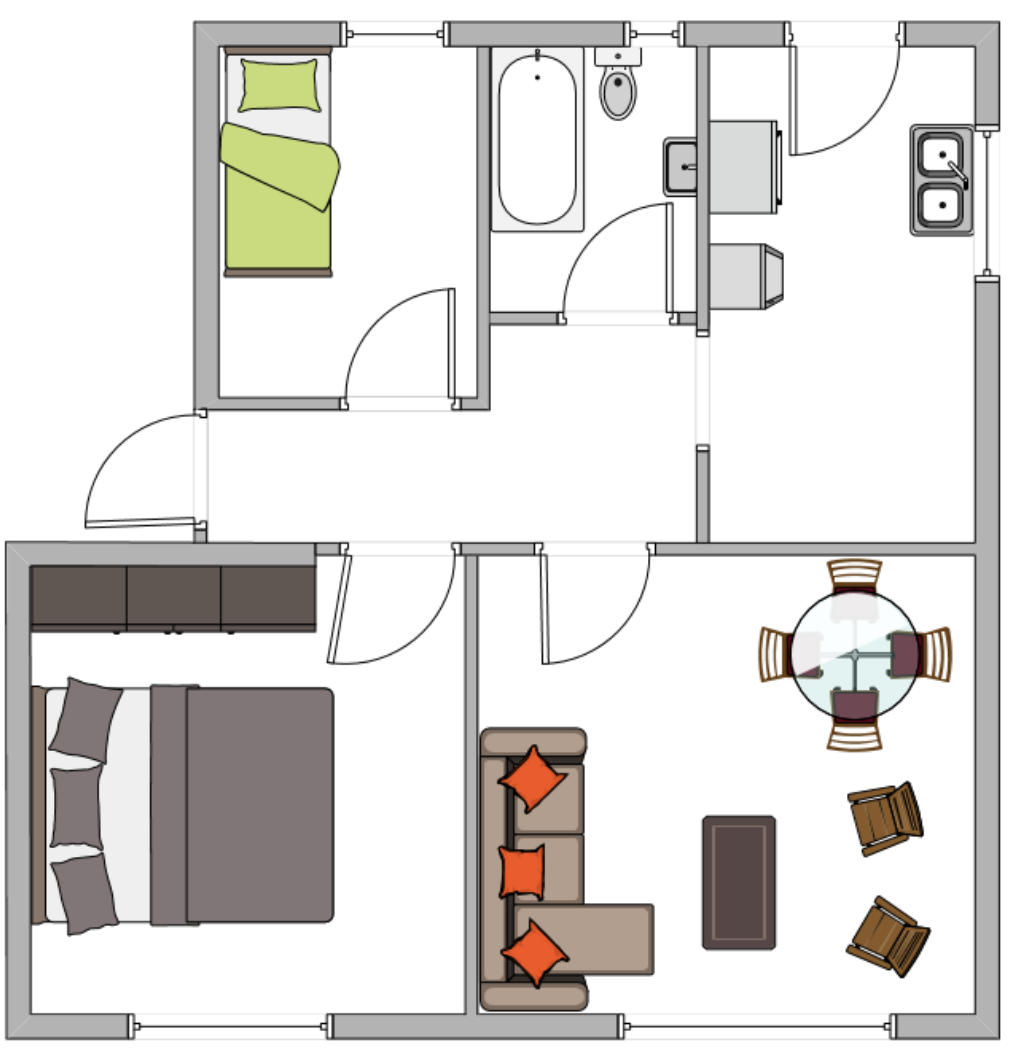

Figure 20: Floorplan of the dwelling modelled through the new simulation tool 
The person living in the dwelling, on the other hand, is concerned with five perceptions: thermal sensation (TS), clothing annoyance (e.g., having too much or too little clothing, Clo), loudness $(L)$, brightness of the space $(B r)$ and the desire of reducing of utility bills $(U B)$. Note that the perceptions associated with daylight-as shown in Table 6-have been summarized in a single "brightness" perception. The reason for this is that, as discussed in Section 5.2.3, the different perceptions identified as relevant for daylight (e.g., attractiveness and cleanliness of the space) may be actually different ways of expressing a single perception that depends only on the "brightness" of a space.

Equation 10 shows the satisfaction function for this individual. While this function was arbitrarily designed, it respects the positive or negative nature of the perceptions. For instance, positive brightness always increases satisfaction and a positive utility bill always decreases it. Similarly, thermal sensation (TS), clothing annoyance $(C l o)$, and loudness $(L)$ are all squared because their best possible value is 0.0 , and they all reduce people's satisfaction when being positive or negative. (This is not meant to suggest that thermal sensation, clothing annoyance or loudness dominate people's Feeling of Comfort). Developing this proof of concept model to a more sophisticated representation of the perceptions is the future research necessary for further development of this concept.

$$
s(t)=5 B r-2 T S^{2}-1.5 C l o^{2}-2 L^{2}-0.4 U B
$$

People's awareness of the future was modelled according to Equation 3. The parameters utilized indicate that people care up to three hours in the future and that the awareness starts decaying right away (i.e., in equation 3, this means that the parameters $h_{1}$ and $h_{2}$ have been set to 0 and 3 hours, respectively).

\subsubsection{Simulating Trade-offs, Perceptions and Expected Outcomes}

As explained in Section 5.2.3, this dissertation proposes that Perceptions, Trade-offs and Expected Outcomes are the determinants of the Feeling of Comfort. Consequently, the simulation tool described in this chapter was designed to incorporate these three elements. Figure 21 shows the results of a simulation performed employing a prototype simulation tool developed according to what has been described in this chapter. Figure 21 evidences that this prototype tool was capable of simulating Perceptions, Trade-offs and Expected Outcomes.

Figure 21 not only shows that this new simulation tool is considering multiple physical (i.e., lighting, thermal and acoustic) and non-physical (e.g., utility bills and clothing annoyance) domains simultaneously, but that they interact with each other at runtime. For 
instance, it reveals that, when the person puts clothes on, his/her thermal sensation and clothing annoyance change without affecting the concern for high utility bills. Similarly, turning on a heater affects the thermal sensation and the utility bills, all this while not affecting anything else.

Another element to notice in the results is that the person's loudness perception starts to decay about three hours before the noisy rush hour (see Figure 19 for details on the external noise). This happens because the person being simulated has an awareness of three hours (modelled according to Equation 3). It is worth mentioning that this does not mean that the person is indeed hearing any noise, but only that his/her mind is already concerned about it.

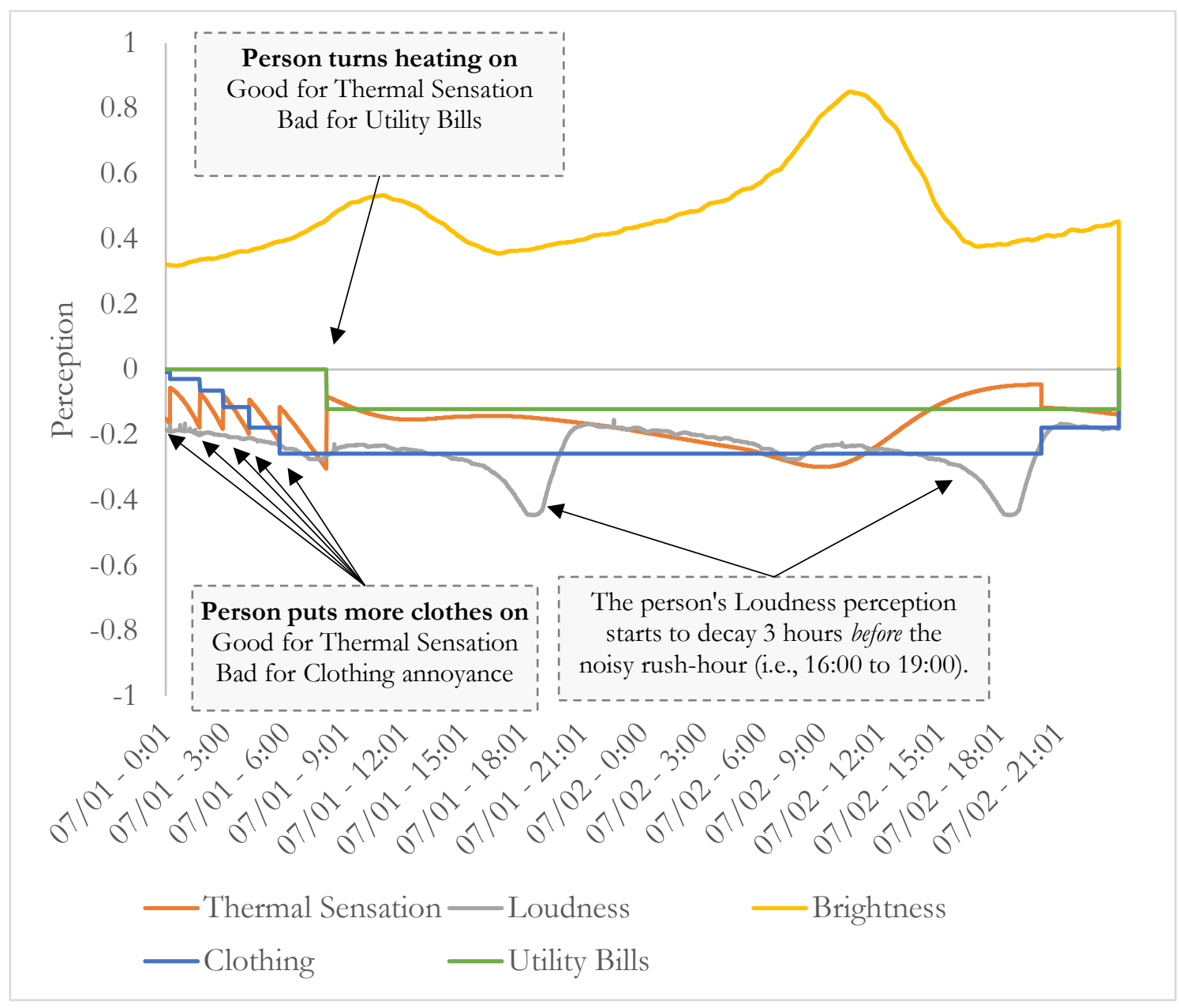

Figure 21: Perceptions felt by the person living in the dwelling according to the simulation

\subsubsection{The effect of Personal Control on people's comfort}

It is well-known that people's control over their environment affects their Feeling of Comfort (Lolli et al., 2020; Luo et al., 2014; Veitch \& Newsham, 2000; Zhou et al., 2014). As explained in Section 5.2.3, the model of comfort introduced by this dissertation explains this phenomenon through the concept of Expected Outcomes. Specifically, it states that, since 
people know they can fix potentially uncomfortable situations (e.g., heating to prevent cold), their mind is less troubled and therefore they feel more comfortable. Figure 22 evidences that the Simulation Tool introduced in this chapter is capable of representing this phenomenon.

These are the results of two distinct simulations. In one of them, the person is free to change his/her clothes and also every room has a heater and luminaires. On the other case, on the contrary, the simulated individual cannot change their clothing, there are no luminaires or heaters in the dwelling, and no windows are openable. Additionally, the simulation was set up so neither of these two individuals ever engaged in any behaviour by setting their Indifference ( $\pi$, in Equation 9 in Section 7.6) to a very high number. This means that the only difference between both simulations is what the individuals know they can do, not the actual physical conditions of the dwelling.

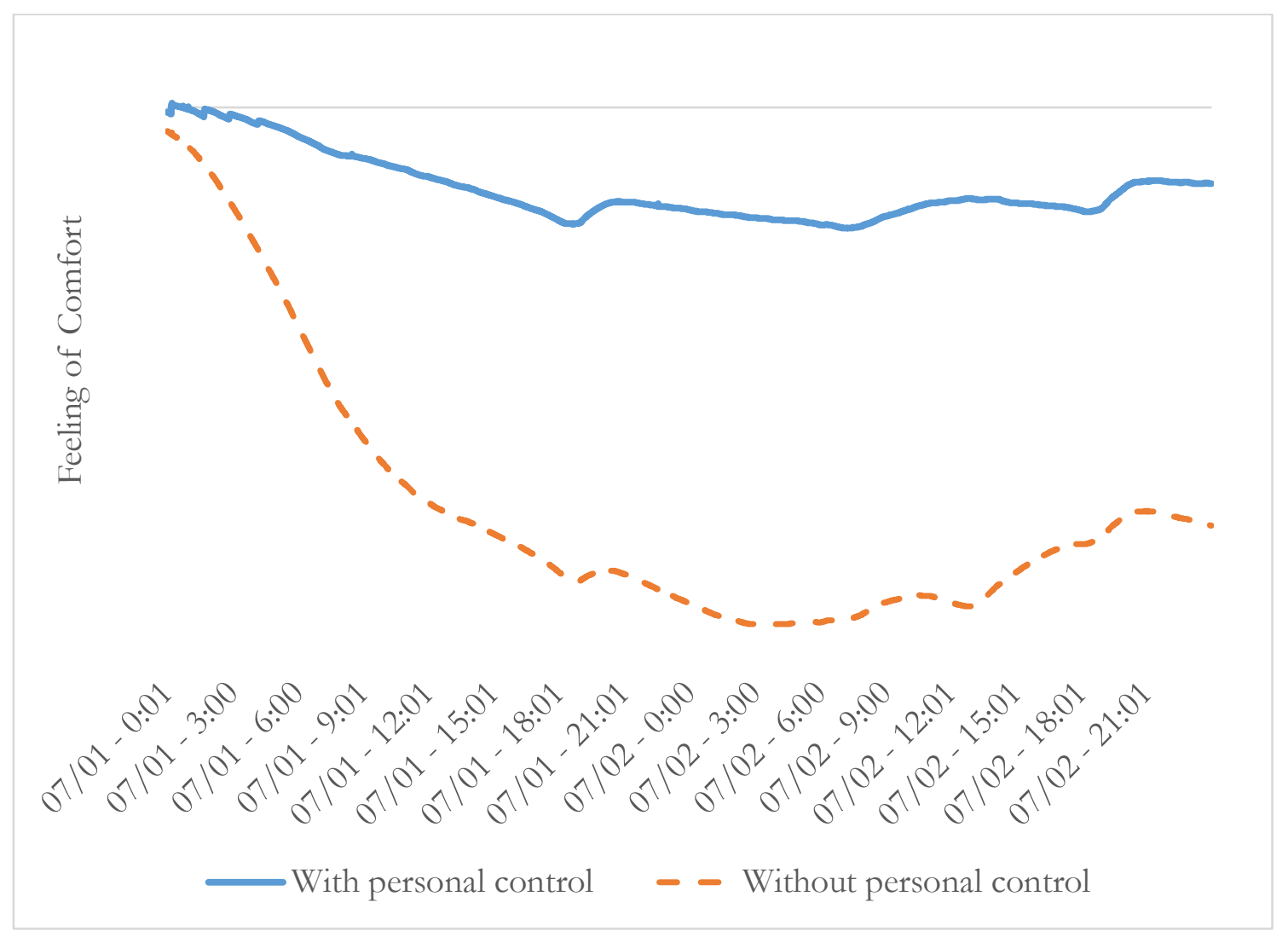

Figure 22: How personal control affects the Feeling of Comfort according to the simulation

\subsubsection{Simulating attention}

As mentioned in Section 5.2.2, the development of the Feeling of Comfort only happens when a situation captures people's attention. Equation 8 models the probability of a person attending a situation $(\alpha)$ as a function of the satisfaction with the environment $(S)$, and a busyness parameter $(\beta)$. That equation states that the probability of attending a situation is greater when said situation becomes more unpleasant, and lower when the person is busy. 
The implication of Attention being a prerequisite for Feeling Comfort—explained in Sections 5.2.2 and 6.2.2 - is that, even if they are exposed to situations whose environmental conditions would allow estimating comfort indices, people very often feel neither comfortable nor uncomfortable. Sometimes the Feeling of Comfort simply does not develop. Figure 23 evidences this, showing two simulations: one in which the person is busy and another one in which he/she is not. The results from the simulation show how the person who is not busy continuously develops a Feeling of Comfort, the one who is busy does not.

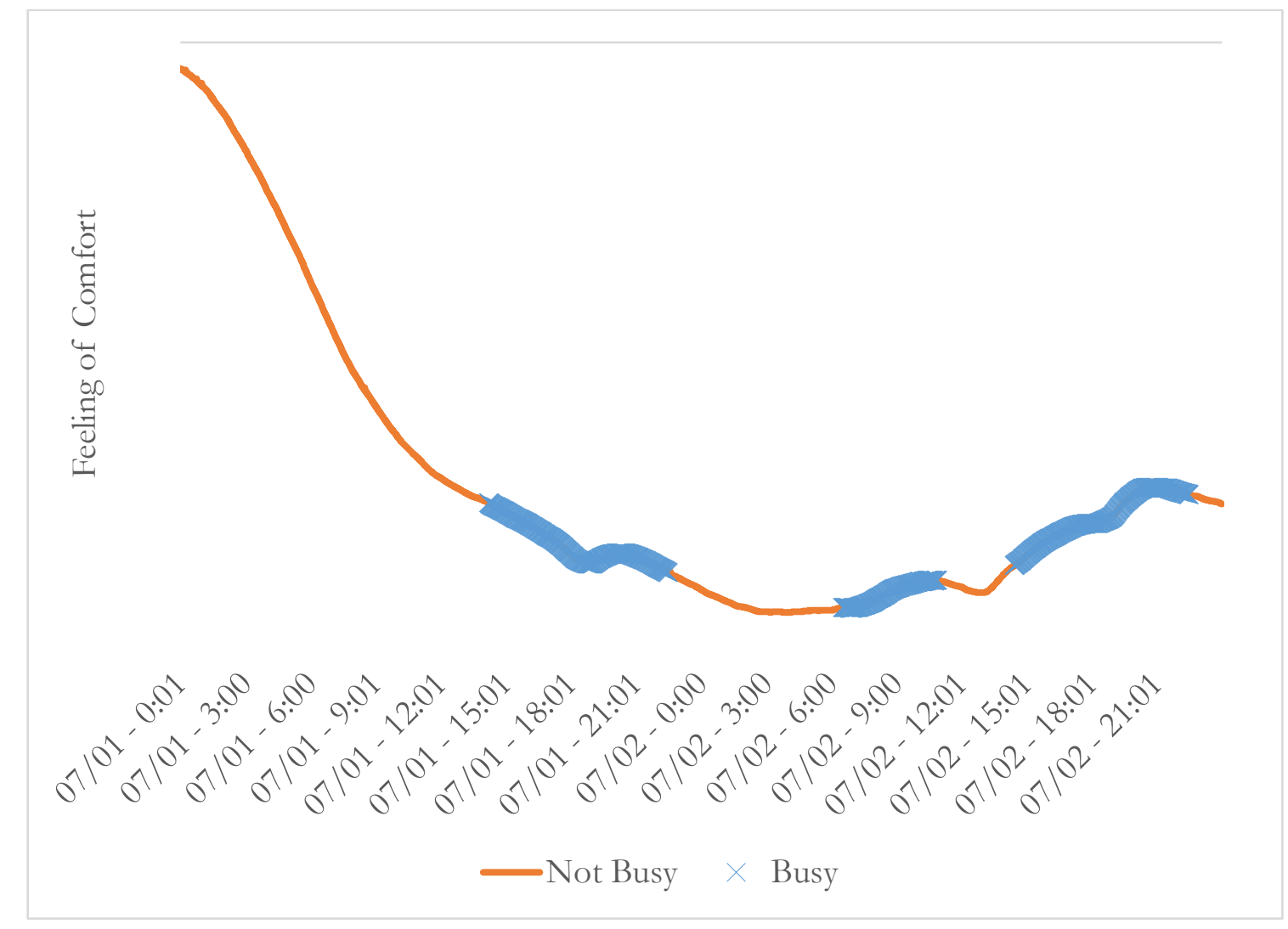

Figure 23: How busyness affects the Feeling of Comfort according to the simulation

\subsection{Conclusions and chapter summary}

After introducing a qualitative model of the Feeling of Comfort (chapters 5 and 6) which was then represented through a set of equations (Chapter 7), this chapter examined whether building performance simulation tools could incorporate the Feeling of Comfort. An iterative process of prototyping and testing allowed identifying an architecture that makes it possible for building performance simulation tools to do so. This chapter discussed this architecture and the results of simulations performed with a prototype tool that uses it. These results show that the Feeling of Comfort and the related behaviours can be plausibly modelled.

It was found during the development process that any building performance simulation 
tool that attempts to model the Feeling of Comfort must comply with two requirements. First - since people do not strictly separate the thermal, lighting, acoustic and air quality domains - it must be able to simulate multiple physical domains simultaneously and at runtime. And second-since people's Feeling of Comfort not only depends on the present but also the future-it must be able to move towards the future and then come back to the present. An architecture that allows simulation tools to comply with these requirements was also described in this chapter.

The results produced by this tool (and shown in this chapter) evidence how it can more appropriately emulate people's Feeling of Comfort than other traditional tools. Specifically, this new tool can not only model Perceptions - the main focus of most traditional toolsbut also Trade-Offs. This is possible because it accounts for multiple domains simultaneously and at runtime during the simulation. Additionally, since this tool is capable of going back in time (i.e., to simulate some hours into the future and then jump back into the present), this tool can emulate peoples' expected outcomes. Specifically, it was shown how this tool can potentially reproduce the beneficial effect that personal control has over people's Feeling of Comfort. 


\section{CONCLUSIONS, IMPLICATIONS AND FURTHER RESEARCH}

'If you ask a bunch of scientists 'how would you like to live 400 years?' everyone says 'Yay!'. And if you ask them why, they say 'Well, I am working on a problem that I might not have time to solve. But if I had 400 years, I bet I could get somewhere on it"”

MARVIn Minsky, THE SOCIETY OF Mind (LECTURE 3, FALL 2011)

This thesis has argued that there is a contradiction within building science's comfort research. Specifically, while this discipline mostly introduces comfort as a subjective state-of-themind (Altomonte et al., 2020; e.g., Auliciems, 1981, 1983; de Dear et al., 2016), its research methods, models, and conceptual frameworks avoid the subject and "the mind" as much as possible by disregarding cognition and non-physical individual characteristics (e.g., Fountain \& Huizenga, 1996; Jakubiec \& Reinhart, 2012; Sarey Khanie, Stoll, Einhäuser, Wienold, \& Andersen, 2017; van Hoof, Mazej, \& Hensen, 2010; Wienold \& Christoffersen, 2006; Wienold et al., 2019). These two often-disregarded elements-cognition and people's nonphysical personal factors_-is what this dissertation calls "the mind".

The relevance of this research lies in the fact that, according to its results, "the mind" plays a significantly relevant role in how people assess the built environment and thus disregarding it can greatly limit the validity of building science's comfort research and practice. Moreover, this research demonstrates that it is possible to mitigate this issue by proposing a new qualitative Feeling of Comfort model that embraces "the mind". This model was introduced in Chapter 5 and the results of the study that helped develop it were replicated in Chapter 6. This model can be used for guiding future comfort research and practice. For instance, it allowed developing a quantitative model of the Feeling of Comfort (Chapter 7) which was incorporated into a prototype building performance simulation tool (Chapter 8).

This thesis also responded to the three research questions proposed in Section 3.2. The answers to these questions are available in chapters 5 to 8 . Specifically, Chapters 5 and 6 contain the answers to questions 1 and 2, asking about the nature and the role of "the mind" in the determination of people's Feeling of Comfort. Similarly, Chapters 7 and 8 contain the 
answer to question 3, related to how quantitative methods commonly used in building science can incorporate "the mind". Having answered these questions, this chapter focuses on outlining the main conclusions, findings, and implications of this research. It also discusses the paths for future research that can branch from this dissertation.

\subsection{Conclusions about the research problem}

This research addressed a contradiction that exists within building science: while comfort is mostly defined as a subjective state of the mind (Altomonte et al., 2020), the methods and conceptual frameworks that drive building science's research and practice tend to disregard "the mind" and to oversimplify the subject. An important characteristic of this contradiction is that, as stated in Section 2.3, it does not seem to be rooted in ignorance but in the fear that embracing these elements might make building science's comfort research and practice overwhelmingly complex.

This dissertation furthers the understanding of this research problem in three ways. First, it suggests that embracing "the mind"-i.e., cognition and non-physical personal factorswould be not only beneficial for building science's research and practice but also crucial for designing buildings that truly respond to people's expectations and needs in terms of comfort. Second, it discredits the belief that embracing "the mind" will result in chaos. On the contrary, it suggests that it is possible to develop theories, conceptual frameworks and models of comfort that are simple enough to drive building science's research and practice. And third, this dissertation posits that embracing "the mind" requires tools and research methods that have not traditionally been used in building science.

\subsubsection{Embracing "the mind" is crucial}

This research suggests that embracing "the mind"-i.e., people's non-physical personal factors and their cognitive processes-would be not only beneficial for the discipline of building science but that it is also critical. The reason for this is that, according to the results of this research, cognition and people's non-physical personal factors play a significantly relevant role in how people assess the built environment.

Regarding cognition, Chapters 5 and 6 identified three main appraisals (i.e., cognitive processes) that respondents seem to use for assessing how comfortable a situation is. Specifically; Perceptions indicate whether people find the here and now pleasant; Expected Outcomes, determine whether they think the future will be pleasant; and Trade-Offs, reflect whether they have made any subjectively significant sacrifices to achieve their current state of comfort. 
It is worth mentioning that building science has managed to study Perceptions by treating them as Sensations; that is to say, by disregarding the fact that the former is the meaning people give to the latter (Coren et al., 2004; de Dear, 2011). However, it is virtually impossible to study Trade-Offs and Expected Outcomes without embracing "the mind" because these two appraisals are nearly impossible to associate with people's physiological systems. Furthermore, ignoring cognition implies consolidating the concepts of Perceptions and Sensations and this research suggests that doing this is inappropriate for two reasons.

First, respondents of this research expressed how the same environmental conditions can be given a different meaning at different times. For instance, respondents stated that, depending on their source, they judge equally loud noises differently. Cracking noises in timber floors and doors are associated with wear and tear. Noises from toilets and pipes, on their part, suggest a lack of privacy. The noise of nature, on the contrary, implies quiet, calm and peace. This is consistent with previous literature suggesting that people interpret noise differently depending on what it represents (Angevine, 1972; Vardaxis \& Bard, 2018b). Likewise-regardless of them being extremely similar-respondents expressed how electric light is not a good substitute for daylight. Specifically, they expressed that, while electric light only allows them to operate properly within the dwelling, daylight also means vitality, happiness, health, and energy. The fact that equal (or very similar) environmental conditions can result in drastically different judgments reveals how inappropriate it is to consolidate perceptions and sensations into a single concept that depends mostly on physical elements.

The second reason that perceptions as sensations should not be confused is that some perceptions do not seem to be strongly related to sensations. On the contrary, this research identified perceptions that are rooted mostly in cognition and that depend only weakly on people's physiology. For instance, respondents revealed how they feel uncomfortable bothering others (associated with acoustics and air quality) or by being exposed to others who can see and/or hear them. Building science seems to have scarcely studied this kind of cognition-driven perception. On the contrary, the perceptions that have been studied are mainly those that can be easily associated with sensing organs. For instance, glare (Jakubiec \& Reinhart, 2012; Konstantzos \& Tzempelikos, 2017; Sarey Khanie et al., 2017; Sawicki \& Wolska, 2015; Wienold, 2009; Wienold \& Christoffersen, 2006), thermal sensation (ASHRAE, 2010; ISO, 1984), and loudness (Egan, 1988).

The importance of considering people's non-physical factors lies in the fact that they shape the meaning people give to the situations they are in. They are the starting point for the development of a Feeling of Comfort. For instance, respondents' household 
composition seems to be relevant because they expressed how their comfort did not depend only on themselves but also on what they think other people are experiencing. This effect is accentuated if these other members of their household are particularly sensitive to environmental conditions. Likewise, respondents' mentioned having a certain-not necessarily specific or clear-budget for utility bills associated with comfort. This budget, however, does not seem to depend exclusively on the respondents' incomes. Evidence of this is that-even if none of the participants of this research happened to be in a demographic segment associated with energy poverty—some of them mentioned that they are willing to pay whatever it takes to keep their homes comfortable, while others would rather sacrifice comfort as long as it keeps the bills within budget. People's budgets seem to be more associated with "mental accounting" (Thaler, 1999), meaning that people group expenditures into categories (i.e., accounts) and control the budget of each of them separately.

It is worth mentioning that non-physical personal factors have mostly been disregarded by building science's comfort research. This is undeniable for models of comfort that are exclusively based on human physiology and physics (e.g., ASHRAE, 2010; Wienold \& Christoffersen, 2006). However, this also seems to be true for models of comfort that incorporate some psychological and/or cognitive elements. This is made possible by, instead of trying to understand the role of different personal factors, treating variability between different people as statistical error or uncertainty (O’Brien et al., 2017). For instance, by assuming that "there will always be a percentage of dissatisfied occupants" (ISO, 1984, p. 4) and thus the goal is to "specify environments known to be acceptable by a certain percentage of the occupants." (ISO, 1984, p. 4). The benefit of identifying the most relevant nonphysical personal factors is that they can be used to create subgroups and thus develop comfort models that, even if they are not fully personalized, can better represent certain groups of the population. In other words, since the variability between people within the same group would be smaller, these models would very likely be able to satisfy larger percentages of the population.

Consequently, ignoring "the mind" necessarily implies ignoring two and a half ${ }^{10}$ out of three appraisals respondents revealed using for determining how comfortable a situation is. Also, ignoring "the mind" implies disregarding the characteristics that determine the meaning people give to the situations they are in. Thus, conceptual frameworks, theories and models of comfort that ignore these factors fail to represent properly the concept of comfort held

\footnotetext{
${ }^{10}$ Because some Perceptions cannot be easily linked to physiology
} 
by the people these buildings are designed for. Designing dwellings by ignoring these factors is likely to result in a significant discrepancy between the level of comfort estimated by standards and calculations and the one experienced by the people who live in them.

\subsubsection{It is not true that embracing "the mind" will necessarily make comfort research overwhelmingly complex}

This dissertation is evidence of how embracing "the mind" will not necessarily make comfort research and practice overwhelmingly complex. That is to say, this research simultaneously embraced "the mind" and successfully gathered, made sense, and utilized qualitative techniques for developing two models of the Feeling of Comfort. These modelsone qualitative (see Chapters 5 and 6) and one quantitative (see Chapter 7)—proved to be simple enough to allow performing building performance simulations (see Chapter 8).

The reason why embracing "the mind" does not lead to chaos seems to be that, even if in theory every person might hold a unique understanding of comfort, in practice people tend to agree with each other. For instance, it is possible to think of an individual who finds it comfortable to live in a humid and dark dwelling maintained at $43^{\circ} \mathrm{C}$ or more. In practice, however, finding this person is likely to be extremely challenging. The extent to which people agree with each other can be deduced from Figures 4 to 8 , which show that only a handful of interviews are required for reaching theoretical saturation. In other words, those figures evidence that the amount of new information gathered by each interview quickly decays after interviewing 10 to 15 people. Consequently, interviewing more people would make little difference in an exploratory analysis like the one performed in this research. This is consistent with several studies in different disciplines (Bowen, 2008; Corbin \& Strauss, 2008; Francis et al., 2010; Rowlands et al., 2015).

\subsubsection{Embracing "the mind" requires tools and research methods that have not been traditionally used in building science}

Unfortunately, embracing "the mind" in comfort research and practice does not seem to be an incremental step. On the contrary, since comfort is an intrinsically qualitative concept, qualitative research methods and analyses-which have not been historically used by building scientists—are required for studying it. It is only after a relatively deep understanding of this qualitative phenomenon is achieved that quantitative models of comfort can be produced and validated. While only a starting point, this dissertation followed this path (see Section 4.3).

Furthermore, this research suggests that people always put comfort in a broader context (see Trade-Offs in Section 5.2.3). This means that, as opposed to many building science 
researchers and practitioners (e.g., Andargie et al., 2019; Bluyssen, 2009), people do not strictly separate different domains of indoor environmental quality and they do not strictly separate indoor environmental quality from the rest of their lives. This undermines the usefulness of building performance simulation tools and of simulation and optimization studies that focus exclusively on one or two indoor environmental quality domains (e.g., Energy Systems Research Unit, 2021; Guglielmetti et al., 2011; Jakubiec \& Reinhart, 2011; Lawrence Berkeley National Laboratory, 2013; Molina, 2018; Roudsari \& Pak, 2013; Ward \& Shakespeare, 1998). This thesis suggests that building science needs to normalize a more holistic approach to comfort research and practice.

Apart from stressing the need for a change in building science's comfort research and practice, this dissertation argues that building scientists need a new kind of building performance simulation tools. The reason for this is twofold. The first one relates to the fact that comfort research and practice needs to be more holistic. Consequently, any building simulation tool that aims to help perform comfort research needs to account for all the indoor environmental quality domains and even other elements beyond building physics. The second reason is associated with the fact that-according to this research-people's comfort depends not only on the present and the past but also on the future (see expected outcomes in Section 5.2.3). Consequently, apart from being able to perform holistic analyses, a tool attempting to help to perform comfort research must be able to, at each time step, infer the future and come back to the present. Unfortunately, there seems to be no existing building performance simulation tool that complies with these two characteristics. Despite this, this dissertation showed that it is possible to develop such a tool by producing a prototype of one (see Chapter 8).

\subsection{Implications for policy and practice}

This research evidences how important it is to embrace "the mind"-i.e., people's nonphysical personal factors and their cognitive processes—when performing comfort research and practice. In other words, this thesis suggests that it is of crucial importance to always distinguish between indoor environmental quality and comfort. In practice, this implies remembering that the models of comfort-embedded in building performance simulation tools and represented in standards-tend to only cover perceptions. Thus, even if this thesis does not attempt to underestimate their value, it is important to always keep in mind that these models of comfort only cover one of the three appraisals that determine people's Feeling of Comfort (see Chapters 5 and 6).

An implication that follows the point above is that, since people's Feeling of Comfort 
goes beyond indoor environmental quality (see trade-offs in Section 5.2.3), then building codes should not focus exclusively on building physics. For instance, if dwellings are to be built in locations where insects or wind might prevent people from opening the windows, this should be taken into consideration. This implies that, if the building code expects people to open the windows, then a solution for the insect and/or the wind issue should be proposed. Alternatively, it may be necessary to design a building that does not expect people to open the windows and modify the requirements accordingly. The same happens with people's safety concerns and traffic noise, which can also prevent people from opening windows. Consequently, building codes should either deal with these problems or they should not rely on people opening their windows. Similarly, since the noise caused by the ventilation systems (e.g., the hood in the kitchen) can prevent people from turning them on, building codes that expect people to use these systems should not only mandate that they are installed but also that they are quiet enough to avoid disturbances. This principle applies to virtually all Trade-Offs in Tables 8 and 15 and also touches other aspects of people's interactions with the built environment. For instance, this research identified that some people do not trust mechanical ventilation because they feel it is less effective than opening windows. Hence, a building code that expects people to use these systems should be accompanied by an understanding of this lack of trust, and solutions to it.

A similar insight can be offered to practitioners. That is to say, while building performance simulations are useful for evaluating design alternatives, the modelling assumptions and the analysis of the results should always consider elements beyond building physics. For example, it is not sensible to model occupants that open windows at times in which insects or noise or safety concerns are known to prevent that from happening. From this perspective, building performance simulation tools-not just the one introduced in Chapter 8 but also the current ones - can be used to evaluate design decisions that seem, at first, unrelated to building physics. For example, by changing how people are assumed to behave (e.g., opening windows or not), it is possible to evaluate how a dwelling's performance would improve after installing flyscreens (i.e., by having or not a solution for the insect problem).

Finally, since people's Feeling of Comfort depends not only on measurable factors, the results of this research suggest that talking to people is the best way of assessing how comfortable their homes are. This is advantageous from a policy-making perspective for two reasons. First, because it is easier and more cost-effective than monitoring the environmental quality (e.g., measuring illuminance, temperature, relative humidity) for extended periods of time. And second, because it also captures the effect of non-observable 
and non-measurable factors (e.g., expected outcomes and trade-offs). Furthermore, it is recommended that —at least at the first stages of this kind of investigation (e.g., in the process of developing a standardized questionnaire)_people are allowed to express their opinion freely (as opposed to asking them questions purely through Likert-scale questionnaires, as commonly recommended, e.g., Stevenson, 2019). The purpose of this is to add an exploratory stage that can identify the kind of responses people are likely to provide and thus can help to develop an adequate questionnaire (e.g., Francis et al., 2004).

\subsection{Implications for methodology}

This section outlines two main kinds of implications associated with research methods and methodology. The first one is a reflection of the specific method utilized. This research utilized qualitative data gathered through semi-structured interviews in which non-expertspeople with no knowledge of the current theories of comfort (i.e., not building scientists, engineers or architects, etc.) — were allowed to speak freely. They were asked to describe a comfortable home on their own terms. While unusual for the discipline of building science, this method proved to be useful for performing comfort research.

The second kind of implication derives from the results of this research, which raise several concerns about the techniques that building science currently uses for investigating comfort. This does not mean that all of the historically utilized methods should be avoided, but some considerations should be taken into account when interpreting their results.

\subsubsection{Reflections on the method utilized}

Perhaps the most important lesson from the methodology used in this research is that exploratory and qualitative comfort research is a valuable and powerful research approach. As evidenced by this research, this approach was capable of providing insights on how people think about comfort and helped identify perceptions that, even if they have virtually never been considered by building scientists, might be relevant in the determination of people's Feeling of Comfort. It revealed, for instance, that people perceive daylight as being beneficial for their health and wellbeing, and that it is preferable to electric light (see Chapter 5). These are well-known scientific facts today (Berson, Dunn, \& Takao, 2002; Beute, 2014; Gochenour \& Andersen, 2009; Zeitzer, Dijk, Kronauer, Brown, \& Czeisler, 2000). The agreement between people's responses and the scientific evidence suggests that asking non-experts about their experiences with the built environment can be a good exploratory starting point for building science's comfort research. The results of these studies can then inform the design of quantitative and confirmatory research. 
After gathering data, it was useful to distinguish between what people said was comfortable and why they think it was comfortable. The rationale behind this is that respondents showed that they were highly knowledgeable about what makes them comfortable and thus their views on this topic should be given considerable attention. This knowledge is different, however, from the theoretical knowledge that would allow them to explain why a building performs in a certain way. Thus, even if they sometimes did offer an explanation (and sometimes it was accurate), this information was not given as much attention.

A final advantage of the methodology adopted in this research was that, by being exploratory, it offered unexpected insights and raised unforeseen questions. This made it possible to capture the concept of comfort held by those individuals for whom buildings are designed, without filtering their responses-through templates and protocols that reflect the researcher's understanding of comfort.

\subsubsection{Implications for currently used methods}

The first implication arising from the results of this research is that, if comfort is a state of the mind, then the data that represents people's behaviour-gathered through sensors or observations-should be interpreted carefully. The reason for this is that people's behaviour does not always lead to a subjectively comfortable indoor environmental quality. On the contrary, while people actions seem to be often motivated by a desire for a better indoor environmental quality, they are also likely to sacrifice it as long as they perceive other benefits. This might be the case, for instance, of people allowing temperatures to go higher or lower than what they would otherwise consider comfortable, motivated by the desire of reducing the cost of utility bills. Similarly, people might sometimes choose idleness in situations with an arguably unpleasant indoor environmental quality as long as they see other benefits. It is thus recommended to combine observation/monitoring studies with some sort of qualitative method that allows capturing the unmeasurable and/or unobservable factors that affect people's comfort and behaviour. For instance, their motivations, aspirations and reasons for doing what they do (e.g., Attia, 2020; Ben \& Sunikka-Blank, 2015).

It follows from the previous paragraph that not all people's actions can (or should) be associated with indoor environmental quality. Thus, methodologies that assume that the latter is the cause of the former might lead to incorrect conclusions. This undermines the usefulness of studies that derive correlations between physical measurements and people's behaviour (e.g., Haldi \& Robinson, 2010) which is a relatively common practice (Gunay, 
O’Brien, \& Beausoleil-Morrison, 2013). Similarly, building performance simulation tools tend to enforce this assumption-i.e., that people's behaviour is triggered by environmental conditions alone-because, unless some special approach is taken, they only allow modelling occupant behaviour based on the data these tools produced during the simulation, which happens to be physical (e.g., Bustamante et al., 2017; Molina et al., 2014; Reinhart, 2004; Vera et al., 2016). This assumption gets even stricter when using building performance simulation tools that only account for a single domain, as these tools will also enforce the assumption that people's actions are motivated by a specific domain within indoor environmental quality.

The last implication for future researchers to consider is that, according to this research, people are not constantly thinking about comfort. On the contrary, they only develop a Feeling of Comfort once they attend to the situation they are in. This is important because surveys-probably the most utilized technique for gathering data representing people’s comfort (Földváry Ličina et al., 2018; Nicol \& Humphreys, 2002; Nicol et al., 2012; Schweiker, Ampatzi, et al., 2020; Schweiker et al., 2017; Wienold \& Christoffersen, 2006)— force attention and thus also the development of their Feeling of Comfort. This introduces great methodological challenges. On the one hand, asking people to assess their situation affects the natural development of their Feeling of Comfort; and on the other, not asking them and relying only on their behaviour can lead to incorrect interpretation of the data because people's actions are not always motivated by a desire for a better indoor environmental quality. A potential workaround to this would be to treat people's comfort as a latent (i.e., unobserved) variable that mediates between the observed situation and people's behaviour. This would imply integrating the model of the Feeling of Comfort with a model of behaviour (e.g., Ajzen, 1985, 1991; Kuhl \& Beckmann, 1985) and then testing this new combined model of behaviour through, for instance, Structural Equation Modelling (Thakkar, 2020).

\subsection{Limitations and opportunities for future research}

Section 1.6 explained the boundaries of this research, all of which offer opportunities for future research.

For instance, by design, only people who intended to purchase a residence in Chile and New Zealand were interviewed for this research. This group of people is not representative of all people belonging to all demographic segments and cultures. However, as explained in Section 1.6, this limitation was addressed in two ways. First, the chances of the results of this dissertation being highly dependent on culture are reduced by interviewing people from two different countries/cultures. And second, the chances of these results being highly 
dependent on the comfort domain being studied are also reduced by performing the replication study whose results are shown in Chapter 6. In any case, further replications studies can be used to assess how/if the results of this research apply to other socioeconomic and cultural contexts. Likewise, and also suggested in Section 1.6, further replication studies can help evaluate whether the results of this research apply to other kinds of buildings (e.g., commercial, institutional, such as schools, prisons and others) and for those situations in which people are visitors (e.g., friends' home, hotel).

It was also mentioned in Section 1.6 that this research was performed from a building science perspective and thus its results are very much dependent on the concept of indoor environmental quality. However, it is not difficult to see how some of these principles (e.g., the effects of trade-offs) could potentially apply to broader connotations of comfort that are not part of building physics (e.g., safety and space layout). This opens the possibility of generalizing or adapting the model of the Feeling of Comfort introduced in this dissertation to factors beyond indoor environmental quality.

Apart from the boundaries mentioned in Section 1.6, some opportunities for future research were identified during this research. For example, the mathematical model presented in Chapter 7 requires calibration and validation. That is to say, the equations presented in said chapter are only placeholders whose purpose is to show that it is possible to represent the Feeling of Comfort (as understood in Chapters 5 and 6) in quantitative terms. While performing this calibration and validation is not considered a limitation but further research, it is possible for new insights to force the model to be significantly restructured.

Also, the model presented in Chapter 7 is limited because, as presented in this thesis, it does not allow for inter-domain effects. Specifically, to enable emulating people's use of heuristics when choosing their actions, Equation 5 introduced the assumption that the different Perceptions affecting people's satisfaction with a room are independent. Therefore, cross-domain effects (e.g., the effect of daylight on people's thermal Sensation) cannot currently be accounted for. It is unclear how limiting this assumption is, or how it affects the predicting power of the model. For example, Chinazzo (personal communication, 27 of February 2019) warns that her results-i.e., the effect of the colour of the light over people's thermal sensation — are valid for extreme colour stimuli and might not be applicable for normal architectural contexts. This limitation can potentially be eliminated by employing a different heuristic algorithm (i.e., one that does not depend on Equation 7). 
Also, Section 7.1 introduced an assumption that needs testing. Specifically, that people's predictions of the short-term future are relatively accurate and thus they can be approximated by what simulation says will happen. Even if Section 7.1 justified this assumption in a relatively extensive way-arguing that, according to Kahneman and Klein (2009), people's intuition can be trusted in this matter-people's predictions cannot be expected to be perfectly accurate. Thus, it is important to verify whether this assumption holds and whether it is a useful approximation for predicting people's Feeling of Comfort and, potentially, their behaviour. It is worth mentioning that this limitation only applies to the results in Chapters 7 and 8 and that the prototype simulation tool introduced in Chapter 8 can potentially help verify the assumption in question. Similarly, this assumption also opens the possibility of studying how accurate are people's predictions in unfamiliar and/or non-residential environments, how these predictions develop, and how people's behaviour differs from that in familiar and residential environments.

The model introduced in Chapter 7 was developed using a bottom-up approach, starting from a function called people's immediate satisfaction with the room (Section 7.2). This function, however, was never properly defined in that process. (Although Section 7.4.2 assumed it to be linear in the parameters.) Thus, it is necessary to properly define one or several (for different populations) functions representing people's immediate satisfaction with the room.

Also related to the model introduced in Chapter 7—specifically, to the inferences people make about the future - an element worth exploring is how does the actual probability of a system failing relates to the perceived probability of failure (Section 7.4.1). Prospect Theory (Kahneman \& Tversky, 1979; Tversky \& Kahneman, 1992) seems to be a good starting point for this research. Likewise, if there is interest in incorporating the model of the Feeling of Comfort with behaviour models, then it is necessary to understand better how people choose their actions when they think that adaptive behaviour is needed. Section 7.4.2 introduced an algorithm that allows selecting one action that can help improve people's comfort, but it is not clear whether it represents how people truly reason.

Other opportunities for further research relate to properly understanding the nature of the perceptions associated with daylight (Table 6), as it is unclear whether they are different magnitudes of the same perception. For instance, people may qualitatively express the brightness of the space as "the space has sufficient daylight" (e.g., low but acceptable brightness), "the space looks clean" (e.g., more brightness), "the space looks attractive" (e.g., even more brightness), and so on. It is also unclear whether these measures depend solely 
on the quantity of daylight (e.g., illuminance or luminance) or if they also vary with contrast and the percentages of direct/diffuse daylight (e.g., Rockcastle et al., 2017; Rockcastle \& Andersen, 2013). Similarly, it seems necessary to study further the more cognitivelydriven perceptions identified in this research (Table 6 and Table 13); for example, the "sense of connection with the exterior" (in the daylight domain), the "feeling of confinement" (in the daylight, air quality and coolness domains), and the perceptions of "bothering others" (in the acoustic and air quality domains). And finally, it is necessary to develop models that allow accounting for the Trade-offs in Table 18. For instance, to develop a model that can estimate the perception of "visual privacy", and the annoyances caused by "wearing an uncomfortable amount of clothing" and by "paying subjectively high utility bills".

\subsection{Utilizing the qualitative Feeling of Comfort model in other contexts}

As mentioned in Sections 1.6 and 9.4, the 42 participants of this research are not likely to be representative of all the people belonging to all demographic segments and cultures. It is, consequently, reasonable to ask whether the Feeling of Comfort model can be appropriately utilized in other contexts and, if so, how. This section elaborates on such a question.

Perhaps the most important implication of the approach used in this research is that it is impossible to guarantee that the model proposed by this dissertation is universally applicable ${ }^{11}$. This means that reusing this model in different cultural, socioeconomic or demographic contexts requires some considerations. Specifically, a verification phase is recommended. For example, people of the specific target population (e.g., people with younger children, elderly, or social housing tenants) can be interviewed to assess whether their understanding of comfort also includes the elements of the Feeling of Comfort model (i.e., those in Figure 10). These attempts can provide new insights that can be used to improve and modify the Feeling of Comfort model to make it, iteratively, applicable to more and more contexts.

Despite this limitation, it is important to notice that not being able to guarantee the universality of the Feeling of Comfort model is not the same as being certain that it is not universal. In fact, nothing in the collected data or the research strategy utilized suggests that the Feeling of Comfort model is not applicable in other contexts (beyond the limitations

\footnotetext{
11 This is why this dissertation has made no such claim. In fact, the only claim related to the universality of this model is made at the beginning of section 5.2, stating that the sub-elements of the model (e.g., glare, floor temperature, etc.) are not universal
} 
explained in Section 9.4). It is possible to say this because, by design, the Feeling of Comfort model was first developed with data contributed by a diverse group of 18 people, including males and females between 27 and 66 years old, from Chile and New Zealand, married and single, renters and owners, with and without children. Such a study was then replicated by recruiting a similarly diverse group of 24 people. In other words, the Feeling of Comfort model proved to be capable of explaining data gathered in two countries, twice. It is, therefore, plausible that the Feeling of Comfort model will allow making sense of a third dataset, gathered in a third country.

It is also important to recognize that the Feeling of Comfort model is not disconnected from currently available knowledge. On the contrary, chapters 5 and 6 present and discuss the results by contrasting them with the literature not only of building science but also of other disciplines. This means that the qualitative model of comfort introduced by this dissertation is coherent with the knowledge of other disciplines, collected and improved by multiple researchers through numerous studies. For example, the concept of the situation is consistent with that of the literature on emotions (Gross, 2013); the element of attention is consistent with the physiological concepts of absolute threshold (Feher, 2012; Schiffman, 2000, p. 24), which suggests that not all stimuli will trigger perceptions. Similarly, people's awareness of the future-represented by the expected outcomes-is at the very core of Economic sciences through the Expected Utility theory (e.g., H. Peters, 2015). This theory has been applied to housing on several occasions (e.g., Clark \& Smith, 1982; Smith \& Clark, 1982) and—even if it has been widely critiqued (e.g., Kahneman \& Tversky, 1979; Marsh \& Gibb, 2011) — the critique is not related to the existence of people's awareness of the future but to their capability of making accurate and unbiased predictions (Thaler, 2018b).

In summary, while nothing guarantees that the Feeling of Comfort model is universally applicable, it can be said that it was able to make sense of data provided by a diverse group of people, that it successfully went through a replication study, and that its insights are coherent with the knowledge of other disciplines. Assuming that the model was applicable, how can it be used in practice? The truth is that it depends on the users and their objectives. It is worth mentioning, however, that the main purpose of the qualitative Feeling of Comfort model is not to allow doing things faster or more effectively but to offer a new approach to comfort that can identify potential issues or opportunities that were previously overlooked.

For instance, the Feeling of Comfort model can help real estate people to create value in the housing market using comfort, a rare marketing strategy (Molina, Johnstone, MacGregor, \& Donn, 2020). Specifically, it can tell them that comfort seems to be a goal in 
itself while energy savings is only a means (i.e., utility bills and energy consumption are Tradeoffs) and thus marketing should emphasize more of the former and less of the latter (Molina, Macgregor, Johnstone, \& Donn, 2021). Also, real estate people from Chile and New Zealand can also utilize the list of Environmental Cues compiled through this research to identify the elements they should show in their marketing images. For example, showing the elements associated with warmth will suggest to homebuyers that the dwelling they are seeing is warm.

The Feeling of Comfort model can also potentially help policy-makers make better decisions. For example (as explained in Section 9.2), the concept of Trade-Off reveals that mandating the installation of ventilation systems might not be an effective policy if the installed devices cause nuisances that might prevent people from using them. Likewise, the concept of Expected Outcomes reveals that, if people were sceptical of the effectiveness of these systems, then they might be more likely to open the windows than to use them. Another example of the effect of Trade-Offs relates to the actual versus the predicted effect of installing insulation in dwellings. Specifically, if the current situation forced people to tolerate low temperatures to keep their utility bills within budget, then insulating will lead people to increase the temperature of their homes, and thus this intervention will not necessarily save energy. While still a good outcome, this is clearly not the expected result of the policy. This effect has been documented by Attia (2020).

This last example allows seeing the value that building modellers can see in the Feeling of Comfort model. That is to say, concepts such as Trade-Offs and Expected Outcomes can help them question their modelling assumptions. For example, identifying the potential factors that can prevent people from opening the windows and/or using heating and cooling devices can help improve how they model occupants' behaviour. This kind of insight can also help improve payback calculations by helping scrutinize the before and after situations. For example, modellers can ask whether it is reasonable to assume that people are currently spending a certain amount of money on heating (i.e., the before situation) and whether it is reasonable to assume that they will not change the set-points of their thermostats after seeing their utility bills reduced.

Finally, architects can use the Feeling of Comfort model to inform their designs. For instance, conversations with their clients can effectively become interviews where they can assess whether their clients' housing aspirations can also be understood through Trade-Offs, Perceptions and Expected Outcomes. Furthermore, such a conversation can help identify the specific elements associated with these factors (e.g., privacy might be more important than daylight in the bedrooms). This information, organized in a model such as Figure 10 
can help architects make design decisions that lead to better homes for their clients. In the case of projects that do not have a specific client but a certain demographic sector, a number of people from such a target population can be interviewed with the same purpose.

\subsection{Closing remarks}

This chapter presented the conclusions of this research, as well as some of their implications, limitations, and the opportunities for future research that derive from them. In broad terms, this dissertation highlights the need for scrutinizing the very meaning of the word "comfort" in building science and proposes one new definition for it. Hopefully, this new definition will also be evaluated and critiqued, justifying further research that proposes other new definitions of comfort and promoting the quest for a better understanding of comfort. 


\section{REFERENCES}

Ajzen, I. (1985). Behavioral Interventions Based on the Theory of Planned Behavior. Action Control, 11-39. https://doi.org/10.1007/978-3-642-69746-3_2

Ajzen, I. (1988). Attitudes, Personality and Behavior. (T. Manstead, Ed.). Milton Keynes : Open University Press.

Ajzen, I. (1991). The theory of planned behavior. Organizational Behavior and Human Decision Processes, 50, 179_ 211. https://doi.org/10.1016/0749-5978(91)90020-T

Akerlof, G. A. (1970). The Market for "Lemons": Quality Uncertainty and the Market Mechanism. The Quarterly Journal of Economics, 84(3), 488. https://doi.org/10.2307/1879431

Altomonte, S., Allen, J., Bluyssen, P. M., Brager, G., Heschong, L., Loder, A., ... Wargocki, P. (2020). Ten questions concerning well-being in the built environment. Building and Environment, 180, 106949. https://doi.org/10.1016/j.buildenv.2020.106949

Andargie, M. S., Touchie, M., \& O’Brien, W. (2019). A review of factors affecting occupant comfort in multiunit residential buildings. Building and Environment, 160(June), 106182. https://doi.org/10.1016/j.buildenv.2019.106182

Andargie, M. S., Touchie, M., \& O’Brien, W. (2020). A Survey of Factors that Impact Noise Exposure and Acoustics Comfort in Multi-Unit Residential Buildings. Canadian Acoustics, 48(3), 25-48. Retrieved from http://dx.doi.org/10.1016/j.soncn.2016.05.005

Andersen, M., Guillemin, A., Amundadottir, M. L., \& Rockcastle, S. (2013). Beyond illumination an interactive simulation framework for non visual and perceptual aspects daylighting performance. In IBPS A.

Angevine, O. L. (1972). Individual differences in the annoyance of noise. The Journal of the Acoustical Society of America, 52(179), 179-179. https://doi.org/10.1121/1.1982126

Arens, E., Zhang, H., \& Huizenga, C. (2006a). Partial- and whole-body thermal sensation and comfort-Part II: Non-uniform environmental conditions, 31(March 2016), 60-66. https://doi.org/10.1016/j.jtherbio.2005.11.027

Arens, E., Zhang, H., \& Huizenga, C. (2006b). Partial- and whole-body thermal sensation and comfort - Part I: Uniform environmental conditions. Journal of Thermal Biology, 31(1-2 SPEC. ISS.), 53-59. https://doi.org/10.1016/j.jtherbio.2005.11.028

Aries, M. B. C., Veitch, J. A., \& Newsham, G. R. (2010). Windows, view, and office characteristics predict physical and psychological discomfort. Journal of Environmental Psychology, 30(4), 533-541. https://doi.org/10.1016/j.jenvp.2009.12.004

ASHRAE. (1999). ANSI/ ASHRAE Standard 62: Ventilation for Acceptable Indoor Air Quality.

ASHRAE. (2010). ANSI/ ASHRAE Standard 55: Thermal Environmental Conditions for Human Occupancy.

ASHRAE. (2021). ASHRAE Global Thermal Comfort Database II. Retrieved August 4, 2021, from http://www.comfortdatabase.com/

Atkinson, R., Flint, F., \& Gilbert, N. (2001). Snowball Research Strategies. Department of Sociology University of Surrey Guildford GU2 5XH United Kingdom., (33), null-null.

Attia, S. (2020). Spatial and Behavioral Thermal Adaptation in Net Zero Energy Buildings: An Exploratory Investigation. Sustainability, 12, 7961. https://doi.org/doi:10.3390/su1297961

Auliciems, A. (1981). Towards a psycho-physiological model of thermal perception. International Journal of Biometeorology, 25(2), 109-122. https://doi.org/10.1007/BF02184458

Auliciems, A. (1983). Psycho-Physiological Criteria for Global Thermal Zones of Building Design. International Journal of Biometeorology (Vol. Complement).

Auliciems, A., \& Szokolay, S. V. (1997). Thermal Comfort. Brisbane: The University of Queensland Printery.

Ayoub, M. (2019). 100 Years of daylighting: A chronological review of daylight prediction and calculation methods. Solar Energy, 194, 360-390. https://doi.org/10.1016/j.solener.2019.10.072

Ayoub, M. (2020). A Review on Light Transport Algorithms and Simulation Tools to Model Daylighting inside Buildings. Solar Energy, 198(January), 623-642. https://doi.org/10.1016/j.solener.2020.02.018

Bago, B., \& De Neys, W. (2018). The smart System 1: Evidence for the intuitive nature of correct responding on the bat-and-ball problem. Thinking \& Reasoning, 0(0), 1-48. https://doi.org/10.1080/13546783.2018.1507949-2

Barrett, L. F., Ochsner, K. N., \& Gross, J. J. (2007). On the automaticity of emotion. In Social psychology and the 
unconscious: The automaticity of higher mental processes (pp. 173-217). New York: Psychology Press.

Bean, R. (2020). Thermal Comfort Principles and Practical Applications for Residential Buildings (1st ed.).

Ben, H., \& Sunikka-Blank, M. (2015). A Socio-Technical Approach To Thermal Comfort and Heating Behaviour in UK Homes. Cisbat 2015, 1-5.

Berson, D. M., Dunn, F. A., \& Takao, M. (2002). Phototransduction by retinal ganglion cells that set the circadian clock. Science, 295(5557), 1070-1073. https://doi.org/10.1126/science.1067262

Beute, F. (2014). Powered by nature: the psychological benefits of natural views and daylight. Technische Universiteit Eindhoven. https://doi.org/10.6100/IR780722

Beute, F., \& de Kort, Y. A. W. (2014). Salutogenic effects of the environment: Review of health protective effects of nature and daylight. Applied Psychology: Health and Well-Being, 6(1), 67-95. https://doi.org/10.1111/aphw.12019

Beute, F., \& de Kort, Y. A. W. (2018). The natural context of wellbeing: Ecological momentary assessment of the influence of nature and daylight on affect and stress for individuals with depression levels varying from none to clinical. Health and Place, 49(February 2017), 7-18. https://doi.org/10.1016/j.healthplace.2017.11.005

Blanchett, D. (2017). The Home as a Risky Asset. Journal of Personal Finance, 16(1), 7-29.

Bluyssen, P. M. (2009). The Indoor Environment Handbook. RIBA Publishing.

Bowen, G. A. (2008). Naturalistic inquiry and the saturation concept: A research note. Qualitative Research, 8(1), 137-152. https://doi.org/10.1177/1468794107085301

Brager, G., \& de Dear, R. (1998). Thermal adaptation in the built environment: a literature review. Energy and Buildings, 27(1), 83-96. https://doi.org/10.1016/S0378-7788(97)00053-4

Braun, V., \& Clarke, V. (2006). Using thematic analysis in psychology. Qualitative Research in Psychology, 3(2), 77-101. https://doi.org/10.1038/sj.ijir.3900760

Braun, V., \& Clarke, V. (2012). Thematic analysis. APA handbook of research methods in psychology, Vol 2: Research designs: Quantitative, qualitative, neuropsychological, and biological. (Vol. 2). https://doi.org/10.1037/13620-004

Buratti, C., Belloni, E., Merli, F., \& Ricciardi, P. (2018). A new index combining thermal, acoustic, and visual comfort of moderate environments in temperate climates. Building and Environment, 139(April), 27-37. https://doi.org/10.1016/j.buildenv.2018.04.038

Burger, J. M. (1989). Negative Reactions to Increases in Perceived Personal Control. Journal of Personality and Social Psychology, 56(2), 246-256. https://doi.org/10.1037/0022-3514.56.2.246

Bustamante, W., Uribe, D., Vera, S., \& Molina, G. (2017). An integrated thermal and lighting simulation tool to support the design process of complex fenestration systems for office buildings. Applied Energy, 198, 36-48. https://doi.org/10.1016/j.apenergy.2017.04.046

Cabanac, M. (1971). Physiological Role of Pleasure. Science, 173, 1103-1107. https://doi.org/10.1126/science.173.4002.1103

Cajochen, C. (2007). Alerting effects of light. Sleep Medicine Reviews, 11(6), 453-464. https://doi.org/10.1016/j.smrv.2007.07.009

Candido, C., \& de Dear, R. (2012). From thermal boredom to thermal pleasure: a brief literature review. Ambiente Construído, 12(1), 81-90. https://doi.org/10.1590/s1678-86212012000100006

Carlucci, S., Bai, L., de Dear, R., \& Yang, L. (2018). Review of adaptive thermal comfort models in built environmental regulatory documents. Building and Environment, 137(March), 73-89. https://doi.org/10.1016/j.buildenv.2018.03.053

Carlucci, S., \& Pagliano, L. (2012). A review of indices for the long-term evaluation of the general thermal comfort conditions in buildings. Energy and Buildings, 53, 194-205. https://doi.org/10.1016/j.enbuild.2012.06.015

Cauwerts, C., \& Bodart, M. (2011). Investigation of 3D projection for qualitative evaluation of daylit spaces. In PLEA 2011 "Architecture and Sustainable development". Retrieved from http://hdl.handle.net/2078.1/156073

Chamilothori, K., Chinazzo, G., Rodrigues, J., Dan-Glauser, E. S., Wienold, J., \& Andersen, M. (2019). Subjective and physiological responses to façade and sunlight pattern geometry in virtual reality. Building and Environment, 150(September 2018), 144-155. https://doi.org/10.1016/j.buildenv.2019.01.009

Cheung, T., Schiavon, S., Parkinson, T., Li, P., \& Brager, G. (2019). Analysis of the accuracy on PMV - PPD model using the ASHRAE Global Thermal Comfort Database II. Building and Environment, 153(December 2018), 205-217. https://doi.org/10.1016/j.buildenv.2019.01.055

Chinazzo, G., Pastore, L., Wienold, J., \& Andersen, M. (2018). A field study investigation on the influence of 
light level on subjective thermal perception in different seasons. Proceedings of 10th Windsor Conference: Retbinking Comfort, 346-356.

Chinazzo, G., Wienold, J., \& Andersen, M. (2018a). Combined effects of daylight transmitted through coloured glazing and indoor temperature on thermal responses and overall comfort. Building and Environment, 144(August), 583-597. https://doi.org/10.1016/j.buildenv.2018.08.045

Chinazzo, G., Wienold, J., \& Andersen, M. (2018b). The Effect of Coloured Glazing on Thermal, Visual and Overall Comfort Evaluation. In AIC Lisboa: colour \& buman comfort. Lisbon, Portugal.

Chinazzo, G., Wienold, J., \& Andersen, M. (2019). Daylight affects human thermal perception. Scientific Reports, 9(1), 1-15. https://doi.org/10.1038/s41598-019-48963-y

Chinazzo, G., Wienold, J., \& Andersen, M. (2020). Influence of indoor temperature and daylight illuminance on visual perception. Lighting Research and Technology, 52(3), 350-370. https://doi.org/10.1177/1477153519859609

Chinazzo, G., Wienold, J., Andersen, M., \& Wienold, J. (2020). Effect of Indoor Temperature and Glazing with Saturated Color on Visual Perception of Daylight. LEUKOS, 00(00), 1-22. https://doi.org/10.1080/15502724.2020.1726182

Clark, W. A. V. (1993). Search and Choice in Urban Housing Markets. In G. and R. G. G. T (Ed.), Behavior and Environment: Psychological and Geographical Approaches (pp. 298-316). Elsevier Science Publishers B.V. https://doi.org/10.1016/S0166-4115(08)60048-5

Clark, W. A. V. (2013). Life course events and residential change: Unpacking age effects on the probability of moving. Journal of Population Research, 30(4), 319-334. https://doi.org/10.1007/s12546-013-9116-y

Clark, W. A. V., \& Lisowski, W. (2018). Examining the life course sequence of intending to move and moving. Population, Space and Place, 24(3), 1-12. https://doi.org/10.1002/psp.2100

Clark, W. A. V., \& Smith, T. R. (1982). Housing market search behavior and expected utility theory: 2. The process of search. Environment and Planning A, 14(1981), 717-737.

Coolen, H. (2008). The meaning of dwelling features: Conceptual and methodological issues. The Netherlands: IOS Press.

Corbin, J., \& Strauss, A. (2008). Basics of Qualitative Research (3rd editio). SAGE.

Coren, S., Ward, L. M., \& Enns, J. T. (2004). Sensation and Perception (6th ed.). John Wiley \& Sons.

Cowan, J. P. (1994). Handbook of Environmental Acoustics. Van Nostrand Reinhold Company.

Crandall, B. W., \& Hoffman, R. R. (2013). Cognitive Task Analysis. In J. D. Lee \& A. Kirlik (Eds.), The Oxford Handbook of Cognitive Engineering (Web). https://doi.org/10.1093/oxfordhb/9780199757183.013.0014

Crawley, D. B., Hand, J. W., Kummert, M., \& Griffith, B. T. (2008). Contrasting the capabilities of building energy performance simulation programs. Building and Environment, 43(4), 661-673. Retrieved from http://www.sciencedirect.com/science/article/pii/S0360132306003234

Crawley, D. B., Lawrie, L. K., Winkelmann, F., Buhl, W. F., Huang, Y. J., Pedersen, C. O., ... Glazer, J. (2001). EnergyPlus : creating a new-generation building energy simulation program. Energy and Buildings, 33, 319-331.

Crawley, D. B., Pedersen, C. O., Winkelmann, F., Witte, M. J., Strand, R. K., Liesen, R. J., \& Buhl, W. F. (2004). EnergyPlus : New, capable and linked. In Proceedings of the SimBuild (Vol. 21). Boulder, CO.

Creswell, J. W. (2014). Research design: Qualitative, quantitative, and mixed methods approaches (4th editio). Sage publications.

Crowley, J. E. (1999). The Sensibility of Comfort. The American Historial Review, 104(3), 749-782.

Cutler, B., Sheng, Y. S., Martin, S., Glaser, D., \& Andersen, M. (2008). Interactive selection of optimal fenestration materials for schematic architectural daylighting design. Automation in Construction, 17(7), 809-823. https://doi.org/10.1016/j.autcon.2008.01.002

de Dear, R. (1998). A global database of thermal comfort field experiments. ASHRAE Transactions. Retrieved from http://search.proquest.com/openview/3954a8858df98e04cf5e76bafffdc1b4/1?pqorigsite $=$ gscholar

de Dear, R. (2004). Thermal comfort in practice. Indoor Air, Supplement, 14(SUPPL. 7), 32-39. https://doi.org/10.1111/j.1600-0668.2004.00270.x

de Dear, R. (2011). Revisiting an old hypothesis of human thermal perception: Alliesthesia. Building Research and Information, 39(2), 108-117. https://doi.org/10.1080/09613218.2011.552269

de Dear, R., \& Brager, G. (2002). Thermal comfort in naturally ventilated buildings: revisions to ASHRAE Standard 55. Energy and Buildings, 34, 549-561.

de Dear, R., Foldvary, V., Zhang, H., Arens, E., Luo, M., Parkinson, T., ... Liu, S. (2016). Comfort Is in the 
Mind of the Beholder: a Review of Progress in Adaptive Thermal Comfort research over the past two decades. In The Fifth International Conference on Human-Environment System. Nagoya.

de Dear, R., Kim, J., \& Parkinson, T. (2018). Residential adaptive comfort in a humid subtropical climateSydney Australia. Energy and Buildings, 158, 1296-1305. https://doi.org/10.1016/j.enbuild.2017.11.028

Dewey, J. (1965). Experience and nature (Kindle Edi). https://doi.org/10.1097/00005053-192701000-00094

Djongyang, N., Tchinda, R., \& Njomo, D. (2010). Thermal comfort: A review paper. Renewable and Sustainable Energy Reviews, 14(9), 2626-2640. https://doi.org/10.1016/j.rser.2010.07.040

Doelle, L. L. (1972). Environmental Acoustics. McGraw-Hill.

Dogan, T., \& Park, Y. C. (2017). A New Framework for Residential Daylight Performance Evaluation. In Building Simulation 2017 (pp. 170-178).

Dogan, T., \& Park, Y. C. (2019). A critical review of daylighting metrics for residential architecture and a new metric for cold and temperate climates. Lighting Research and Technology, 51, 206-230. https://doi.org/10.1177/1477153518755561

Dorizas, P. V., De Groote, M., \& Volt, J. (2018). The inner value of a building: Linking Indoor Environmental Quality and Energy Performance in Building Regulation. Building Performance Institute Europe (BPIE).

Dunning, R. (2016). A Typology of Housing Search Behaviour in the Owner-Occupier Sector. University of Sheffield.

Edwards, W. (1954). The Theory of Decision Making. Psychological Bulletin, 51(4), 380-417. https://doi.org/10.1037/h0053870

Egan, M. D. (1988). Architectural Acoustics. McGraw-Hill.

EN17037. (2018). Daylight in Buildings.

Energy Systems Research Unit. (2021). ESP-r website. Retrieved March 2, 2021, from https://www.strath.ac.uk/research/energysystemsresearchunit/applications/esp-r/

Evans, J. S. B. T. (2003). In two minds: Dual-process accounts of reasoning. Trends in Cognitive Sciences, 7(10), 454-459. https://doi.org/10.1016/j.tics.2003.08.012

Evans, J. S. B. T. (2008). Dual-Processing Accounts of Reasoning, Judgment, and Social Cognition. Annual Review of Psychology, 59, 255-278. https://doi.org/10.1146/annurev.psych.59.103006.093629

Fabbri, K. (2013). Indoor Thermal Comfort Perception (Vol. 83). Springer. https://doi.org/10.1111/ans.12186

Fan, C., Yan, D., Xiao, F., Li, A., An, J., \& Kang, X. (2021). Advanced data analytics for enhancing building performances: From data-driven to big data-driven approaches. Building Simulation, 14(1), 3-24. https://doi.org/10.1007/s12273-020-0723-1

Fanger, P. O. (1989). The new Comfort Equation for indoor Air Quality. ASHRAE Journal, 31(10).

Fanger, P. O., \& Toftum, J. (2002). Extension of the PMV model to non-air-conditioned buildings in warm climates. Energy and Buildings, 34(6), 533-536. https://doi.org/10.1016/S0378-7788(02)00003-8

Feher, J. (2012). Cutaneous Sensory Systems. In Quantitative Human Physiology (pp. 389-399). https://doi.org/10.1016/b978-0-12-800883-6.00035-5

Földváry Ličina, V., Cheung, T., Zhang, H., de Dear, R., Parkinson, T., Arens, E., ... Zhou, X. (2018). Development of the ASHRAE Global Thermal Comfort Database II. Building and Environment, 142(June), 502-512. https://doi.org/10.1016/j.buildenv.2018.06.022

Fontana, A., \& Frey, J. H. (2005). The Interview - From Neutral Stance to Political Involvement. In N. K. Denzin \& Y. S. Lincoln (Eds.), The SAGE Handbook of Qualitative Research (Third, pp. 695-727). SAGE.

Fountain, M. E., \& Huizenga, C. (1996). A Thermal Comfort Prediction Tool. ASHRAE Journal, (September).

Francis, J. J., Eccles, M. P., Johnston, M., Walker, A., Grimshaw, J., Foy, R., ... Bonetti, D. (2004). Constructing questionnaires based on the theory of planned behaviour: A manual for health services researchers. (U. of N. upon T. Newcastle upon Tyne, UK: Centre for Health Services Research, Ed.), Stroke (Vol. 34). Newcastle. https://doi.org/10.1161/01.STR.0000081987.46660.ED

Francis, J. J., Johnston, M., Robertson, C., Glidewell, L., Entwistle, V., Eccles, M. P., \& Grimshaw, J. M. (2010). What is an adequate sample size? Operationalising data saturation for theory-based interview studies. Psychology and Health, 25(10), 1229-1245. https://doi.org/10.1080/08870440903194015

Franzitta, V., Milone, A., Milone, D., Pitruzzella, S., Trapanese, M., \& Viola, A. (2014). A case study to evaluate the indoor global quality. Advanced Materials Research, 864-867, 1054-1058. https://doi.org/10.4028/www.scientific.net/AMR.864-867.1054

Gagge, A. P., Stolwijk, J. A. J., \& Hardy, J. D. (1967). Comfort and thermal sensations and associated 
physiological responses at various ambient temperatures. Environmental Research, 1(1), 1-20. https://doi.org/10.1016/0013-9351(67)90002-3

Gochenour, S., \& Andersen, M. (2009). Circadian Effects of Daylighting in a Residential Environment. In Proceedings LuxEuropa. Istanbul. Retrieved from

http://www.thedaylightsite.com/filebank/Gochenour_Andersen_CircadianLightHousing.pdf

Golasi, I., Salata, F., Vollaro, E. de L., \& Peña-García, A. (2019). Influence of lighting colour temperature on indoor thermal perception: A strategy to save energy from the HVAC installations. Energy and Buildings, 185, 112-122. https://doi.org/10.1016/j.enbuild.2018.12.026

Gross, J. J. (1998). The Emerging Field of Emotion Regulation: An Integrative Review. Review of General Psychology, 2(3), 271-299.

Gross, J. J. (2013). Emotion Regulation: Conceptual and Empirical Foundations. In J. J. Gross (Ed.), Handbook of Emotion Regulation (2nd ed., pp. 3-20). Guilford Publications. https://doi.org/10.1080/00140130600971135

Gross, J. J. (2015). Emotion Regulation: Current Status and Future Prospects. Psychological Inquiry, 26(1), 1-26. https://doi.org/10.1080/1047840X.2014.940781

Guglielmetti, R., Macumber, D., \& Long, N. (2011). OpenStudio: An Open Source Integrated Analysis Platform. In 12th International Conference of International Building Performance Simulation Association (pp. 1-9). Sydney, Australia. Retrieved from http://www.nrel.gov/docs/fy12osti/51836.pdf

Gunay, H. B., O’Brien, W., \& Beausoleil-Morrison, I. (2013). A critical review of observation studies, modeling, and simulation of adaptive occupant behaviors in offices. Building and Environment, 70, 31-47. https://doi.org/10.1016/j.buildenv.2013.07.020

Hacking, I. (2012). Introductory Essay. In University of Chicago Press (Ed.), The Structure of Scientific Revolutions (Fourth Kin). Chicago.

Haldi, F., \& Robinson, D. (2010). Adaptive actions on shading devices in response to local visual stimuli. Journal of Building Performance Simulation, 3(2), 135-153. https://doi.org/10.1080/19401490903580759

Halhoul Merabet, G., Essaaidi, M., Ben Haddou, M., Qolomany, B., Qadir, J., Anan, M., ... Benhaddou, D. (2021). Intelligent building control systems for thermal comfort and energy-efficiency: A systematic review of artificial intelligence-assisted techniques. Renewable and Sustainable Energy Reviews, 144(March), 110969. https://doi.org/10.1016/j.rser.2021.110969

Hellinga, H. (2013). Daylight and View. Journal of Chemical Information and Modeling (Vol. 53). https://doi.org/10.1017/CBO9781107415324.004

Hellinga, H., \& Hordijk, T. (2014). The D\&V analysis method: A method for the analysis of daylight access and view quality. Building and Environment, 79, 101-114. https://doi.org/10.1016/j.buildenv.2014.04.032

Hellwig, R. T., Schweiker, M., \& Boerstra, A. (2020). The ambivalence of personal control over indoor climate - How much personal control is adequate? E3S Web of Conferences, 172. https://doi.org/10.1051/e3sconf/202017206010

Hensen, J. L. M. (1991). On the Thermal Interaction of Building Structure and Heating and Ventilating System. Technische Universiteit Eindhoven.

Heschong, L. (1979). Thermal Delight in Architecture. MIT Press.

Howden-Chapman, P., Crane, J., Matheson, A., Viggers, H., Cunningham, M., Blakely, T., ... Saville-smith, K. (2005). Retrofitting houses with insulation to reduce health inequalities: Aims and methods of a clustered, randomised community-based trial. Social Science and Medicine, 61, 2600-2610. https://doi.org/10.1016/j.socscimed.2005.04.049

Howden-Chapman, P., Matheson, A., Crane, J., Viggers, H., Cunningham, M., Blakely, T., ... Davie, G. (2007). Effect of insulating existing houses on health inequality: cluster randomised study in the community. BMJ, 334(7591), 460. https://doi.org/10.1136/bmj.39070.573032.80

Humphreys, M. A. (1979). The influence of season and ambient temperature on human clothing behaviour. In Proceedings of the First International Indoor climate Symposium (pp. 699-714).

Humphreys, M. A., Nicol, F., \& Roaf, S. (2016). Adaptive thermal comfort: Foundations and Analysis. New York, New York, USA: Taylor \& Francis.

IESNA. (2012). IES Spatial Daylight Autonomy (sDA) and Annual Sunlight Exposure (ASE). New York, New York, USA.

INSUL. (2021). INSUL. Retrieved May 18, 2021, from http://www.insul.co.nz/

Isaacs, N., Camilleri, M., French, L., Pollard, A., Saville-Smith, K., Fraser, R., ... Jowett, J. (2006). Energy Use in New Zealand Households. 
Isaacs, N., Saville-Smith, K., Camilleri, M., \& Burrough, L. (2010). Energy in New Zealand houses: Comfort, physics and consumption. Building Research and Information, 38(5), 470-480. https://doi.org/10.1080/09613218.2010.494383

ISO. (1984). ISO7730:1984(E) Moderate thermal environments - Determination of the PMV and PPD indices and specification of the conditions for thermal comfort.

Jakubiec, J. A., \& Reinhart, C. (2011). DIVA 2.0: Integrating daylight and thermal simulations using rhinoceros 3D, DAYSIM and EnergyPlus. In Proceedings of Building Simulation 2011: 12th Conference of International Building Performance Simulation Association (pp. 2202-2209). Sydney, Australia. https://doi.org/10.1017/CBO9781107415324.004

Jakubiec, J. A., \& Reinhart, C. (2012). The "adaptive zone" - A concept for assessing discomfort glare throughout daylit spaces. Lighting Research and Technology, 44(2), 149-170. https://doi.org/10.1177/1477153511420097

Jakubiec, J. A., \& Reinhart, C. F. (2015). A Concept for Predicting Occupants' Long-Term Visual Comfort within Daylit Spaces. LEUKOS - Journal of Illuminating Engineering Society of North America, 12(4), 185-202. https://doi.org/10.1080/15502724.2015.1090880

Janak, M. (1997). Coupling building energy and lighting simulation. In 5 th International IPBS A Conference. Prague, Czech Republic. Retrieved from http://www.ibpsa.org/\%5Cproceedings\%5CBS1997\%5CBS97_P036.pdf

Johnstone, M.-L. (2017). Depth Interviews and Focus Groups. In Formative Research for Social Marketing: Innovative Methods to Gain Consumer Insights (pp. 67-87).

Johnstone, M.-L., \& Tan, L. P. (2015). Exploring the Gap Between Consumers' Green Rhetoric and Purchasing Behaviour. Journal of Business Ethics, 132(2), 311-328. https://doi.org/10.1007/s10551-0142316-3

Kahneman, D. (2003). Maps of Bounded Rationality: Psychology for Behavioral Economics. The American Economic Review, 93(5), 1449-1475.

Kahneman, D., \& Klein, G. (2009). Conditions for Intuitive Expertise: A Failure to Disagree. American Psychologist, 64(6), 515-526. https://doi.org/10.1037/a0016755

Kahneman, D., \& Tversky, A. (1979). Prospect Theory: An Analysis of Decision under Risk. Econometrica, 47(2), 263-292. https://doi.org/10.2307/1914185

Karmann, C., Schiavon, S., \& Bauman, F. (2017). Thermal comfort in buildings using radiant vs. all-air systems: A critical literature review. Building and Environment, 111, 123-131. https://doi.org/10.1016/j.buildenv.2016.10.020

Kim, Joyce, Schiavon, S., \& Brager, G. (2018). Personal comfort models - A new paradigm in thermal comfort for occupant-centric environmental control. Building and Environment, 132(November 2017), 114-124. https://doi.org/10.1016/j.buildenv.2018.01.023

Kim, Jungsoo, \& de Dear, R. (2012). Nonlinear relationships between individual IEQ factors and overall workspace satisfaction. Building and Environment, 49(1), 33-40. https://doi.org/10.1016/j.buildenv.2011.09.022

Kivunja, C., \& Kuyini, A. B. (2017). Understanding and Applying Research Paradigms in Educational Contexts. International Journal of Higher Education, 6(5), 26. https://doi.org/10.5430/ijhe.v6n5p26

Klabnik, S., \& Nichols, C. (2021). Rust traits. Retrieved February 3, 2021, from https://doc.rustlang.org/book/ch10-02-traits.html

Koklic, M. K., \& Vida, I. (2009). An examination of a strategic household purchase: Consumer home buying behavior. Managing Global Transitions, 7(1), 75-96. https://doi.org/10.1111/j.1470-6431.2010.00953.x

Konis, K. (2017). A novel circadian daylight metric for building design and evaluation. Building and Environment, 113, 22-38. https://doi.org/10.1016/j.buildenv.2016.11.025

Konstantzos, I., \& Tzempelikos, A. (2017). Daylight glare evaluation with the sun in the field of view through window shades. Building and Environment, 113, 65-77. https://doi.org/10.1016/j.buildenv.2016.09.009

Kuhl, J., \& Beckmann, J. (1985). Action control: From cognition to behavior.

Kuhn, T. S. (2012). The Structure of Scientific Revolutions (Fourth Kin). Chicago: University of Chicago Press.

Laszlo, H. E., McRobie, E. S., Stansfeld, S. A., \& Hansell, A. L. (2012). Annoyance and other reaction measures to changes in noise exposure - A review. Science of the Total Environment, 435-436, 551-562. https://doi.org/10.1016/j.scitotenv.2012.06.112

Lawrence Berkeley National Laboratory. (2013). EnergyPlus Engineering Reference The Reference to EnergyPlus Calculations. 
Lawrence Race, G. (2006). Comfort. (J. Roebuck \& K. Butcher, Eds.). London: The Chartered Institution of Building Services Engineers.

Leaman, A., \& Bordass, B. (1999). Productivity in buildings: The "killer" variables. Building Research and Information, 27(1), 4-19. https://doi.org/10.1080/096132199369615

Li, Y., \& Wong, A. S. W. (2006). Psychology and sensory comfort. In Y. Li \& A. S. W. Wong (Eds.), Clothing Biosensory Engineering. Woodhead Publishing Limited.

Liu, Y., Dong, Y., Song, C., Wang, Y., Liu, L., \& Liu, J. (2017). A tracked field study of thermal adaptation during a short-term migration between cold and hot-summer and warm-winter areas of China. Building and Environment, 124, 90-103. https://doi.org/10.1016/j.buildenv.2017.07.022

Lolli, N., Nocente, A., \& Grynning, S. (2020). Perceived control in an office test cell, a case study. Buildings, 10(5). https://doi.org/10.3390/BUILDINGS10050082

Lowry, G. (2018). A comparison of metrics proposed for circadian lighting and the criterion adopted in the WELL Building Standard. CIBSE Technical Symposium, (April), 1-10.

Luo, M. (2020). The Dynamics and Mechanism of Human Thermal Adaptation in Building Environment - A Glimpse to Adaptive Thermal Comfort in Buildings. Springer Theses. https://doi.org/https://doi.org/10.1007/978981-15-1165-3

Luo, M., Cao, B., Zhou, X., Li, M., Zhang, J., Ouyang, Q., \& Zhu, Y. (2014). Can personal control influence human thermal comfort? A field study in residential buildings in China in winter. Energy and Buildings, 72, 411-418. https://doi.org/10.1016/j.enbuild.2013.12.057

Luo, M., de Dear, R., Ji, W., Bin, C., Lin, B., Ouyang, Q., \& Zhu, Y. (2016). The dynamics of thermal comfort expectations: The problem, challenge and implication. Building and Environment, 95, 322-329. https://doi.org/10.1016/j.buildenv.2015.07.015

Luo, M., Ke, Z., Ji, W., Wang, Z., Cao, B., Zhou, X., \& Zhu, Y. (2019). The time-scale of thermal comfort adaptation in heated and unheated buildings. Building and Environment, 151(December 2018), 175-186. https://doi.org/10.1016/j.buildenv.2019.01.042

Mahdavi, A., Berger, C., Amin, H., Ampatzi, E., Andersen, R. K., Azar, E., ... Taheri, M. (2021). The Role of Occupants in Buildings' Energy Performance Gap: Myth or Reality?

March, J. G. (1978). Bounded Rationality, Ambiguity, and the Engineering of Choice. The Bell Journal of Economics, 9(2), 587-608. https://doi.org/10.1016/j.aap.2013.10.028

Mardaljevic, J. (1995). Validation of a lighting simulation program under real sky conditions. Lighting Research and Technology, 27(4), 181-188. https://doi.org/10.1177/14771535950270040701

Marsh, A., \& Gibb, K. (2011). Uncertainty, expectations and behavioural aspects of housing market choices. Housing, Theory and Society, 28(3), 215-235. https://doi.org/10.1080/14036096.2011.599182

Martellotta, F., Simone, A., Della Crociata, S., \& D’Alba, M. (2016). Global comfort and indoor environment quality attributes for workers of a hypermarket in Southern Italy. Building and Environment, 95, 355-364. https://doi.org/10.1016/j.buildenv.2015.09.029

Mauss, I. B., McCarter, L., Levenson, R. W., Wilhelm, F. H., \& Gross, J. J. (2005). The tie that binds? Coherence among emotion experience, behavior, and physiology. Emotion, 5(2), 175-190. https://doi.org/10.1037/1528-3542.5.2.175

Mazzarella, L., \& Pasini, M. (2009). Building energy simulation and object-oriented modelling: review and reflections upon achieved results and further developments. In Eleventh International IBPSA Conference (pp. 638-645). Glasgow.

Mickaël, D., Bruno, B., Valérie, C., Murielle, L., Cécile, P., Jacques, R., \& Severine, K. (2014). Indoor air quality and comfort in seven newly built, energy-efficient houses in France. Building and Environment, 72, 173-187. https://doi.org/10.1016/j.buildenv.2013.10.017

Molina, G. (2014). Integrated thermal and lighting analysis of spaces with controled complex fenestration systems and artificial lighting during the design stage. Pontificia Universidad Católica de Chile.

Molina, G. (2018). GroundhogLighting.com. Retrieved from http://www.groundhoglighting.com

Molina, G., Johnstone, M.-L., MacGregor, C., \& Donn, M. (2020). Disclosing Indoor Environmental Quality to create value in the Residential Real Estate Market. In 26th Annual Pacific Rim Real Estate Society Conference. Canberra.

Molina, G., Macgregor, C., Johnstone, M., \& Donn, M. (2021). What is a comfortable home? — A definition developed from homebuyers' perspective. In 27th Annual Pacific Rim Real Estate Society Conference.

Molina, G., Vera, S., \& Bustamante, W. (2014). A tool for integrated thermal and lighting analysis of spaces with controlled Complex Fenestration Systems and artificial lighting. In eSim 2014. 
Morgan, D. L. (2007). Methodological Implications of Combining Qualitative and Quantitative Methods. Journal of Mixed Methods Research, 1, 48-76. https://doi.org/10.1177/2345678906292462

Morgan, D. L. (2014). Pragmatism as a Paradigm for Social Research. Qualitative Inquiry, 20(8), 1045-1053. https://doi.org/10.1177/1077800413513733

Mujan, I., Anđelković, A., Munćan, V., Kljajić, M., \& Ružić, D. (2019). Influence of indoor environmental quality on human health and productivity - A Review. Journal of Cleaner Production, 217. https://doi.org/10.1016/j.jclepro.2019.01.307

Nabil, A., \& Mardaljevic, J. (2005). Useful daylight illuminance: a new paradigm for assessing daylight in buildings. Lighting Research and Technology, 37(1), 41-57.

Nabil, A., \& Mardaljevic, J. (2006). Useful daylight illuminances: A replacement for daylight factors. Energy and Buildings, 38(7), 905-913. https://doi.org/10.1016/j.enbuild.2006.03.013

Newsham, G. R., Richardson, C., Blanchet, C., \& Veitch, J. A. (2005). Lighting quality research using rendered images of offices. Lighting Research \& Technology, 37(2), 93-112. https://doi.org/10.1191/1365782805li132oa

Nicol, F., \& Humphreys, M. A. (2002). Adaptive thermal comfort and sustainable thermal standards for buildings (Vol. 34).

Nicol, F., Humphreys, M. A., \& Roaf, S. (2012). Adaptive thermal comfort: Principles and practice. New York, New York, USA: Taylor \& Francis.

Nimlyat, P. S. (2018). Indoor environmental quality performance and occupants' satisfaction [IEQPOS] as assessment criteria for green healthcare building rating. Building and Environment, 144(April), 598-610. https://doi.org/10.1016/j.buildenv.2018.09.003

NREL. (2019). raycalls.h source code. Retrieved February 3, 2021, from https://github.com/NREL/Radiance/blob/master/src/rt/raycalls.c

O’Brien, W., Gunay, H. B., Tahmasebi, F., \& Mahdavi, A. (2017). A preliminary study of representing the inter-occupant diversity in occupant modelling. Journal of Building Performance Simulation, 10(5-6), 509_ 526. https://doi.org/10.1080/19401493.2016.1261943

O’Brien, W., Schweiker, M., \& Day, J. K. (2019). Get the picture? Lessons learned from a smartphone-based post-occupancy evaluation. Energy Research and Social Science, 56(June), 101224. https://doi.org/10.1016/j.erss.2019.101224

Ochoa, C. E., Aries, M. B. C., \& Hensen, J. L. M. (2012). State of the art in lighting simulation for building science : a literature review. Journal of Building Performance Simulation, 5(4), 203-233.

Olesen, B. W. (2000). Guidelines for comfort. ASHRAE Journal, 42(8), 41-46.

Park, Y. C., \& Dogan, T. (2019). Adapting the Residential Daylight Score for Arid, Hot, and Humid Climates. In Building Simulation.

Parkinson, T., \& de Dear, R. (2015). Thermal pleasure in built environments: Physiology of alliesthesia. Building Research and Information, 43(3), 288-301. https://doi.org/10.1080/09613218.2015.989662

Parkinson, T., de Dear, R., \& Brager, G. (2020). Nudging the adaptive thermal comfort model. Energy and Buildings, 206, 109559. https://doi.org/10.1016/j.enbuild.2019.109559

Parvaiz, G. S., Mufti, O., \& Wahab, M. (2016). Pragmatism for Mixed Method Research at Higher Education Level. Business \& Economic Review, 8(2), 67-78. https://doi.org/10.22547/BER/8.2.5

Patton, M. Q. (1999). Enhancing the Quality and Credibility of Qualitative Analysis. HSR: Health Services Research, 34(5), 1189-1208. https://doi.org/http://dx.doi.org/10.4135/9781412985727

Peters, H. (2015). Game Theory: A Multi-Leveled Approach (2nd Editio). Springer. https://doi.org/10.1007/9783-662-46950-7

Peters, R. J., Smith, B. J., \& Hollings, M. (2011). Acoustics and Noise Control (3rd editio). Pearson.

Petersen, S., \& Svendsen, S. (2010). Method and simulation program informed decisions in the early stages of building design. Energy and Buildings, 42(7), 1113-1119. https://doi.org/10.1016/j.enbuild.2010.02.002

Pierson, C., Wienold, J., \& Bodart, M. (2018). Review of Factors Influencing Discomfort Glare Perception from Daylight. LEUKOS - Journal of Illuminating Engineering Society of North America, 14(3), 111-148. https://doi.org/10.1080/15502724.2018.1428617

Reinhart, C. (2004). Lightswitch-2002: a model for manual and automated control of electric lighting and blinds. Solar Energy, 77(1), 15-28. https://doi.org/10.1016/j.solener.2004.04.003

Reinhart, C. (2013). http://daysim.ning.com/.

Reinhart, C. (2014). Daylighting Handbook: Fundamentals, Designing with the Sun. (R. Stein, Ed.). 
Reinhart, C., Mardaljevic, J., \& Rogers, Z. (2006). Dynamic Daylight Performance Metrics for Sustainable Building Design. Leukos, 2(1), 7-31. https://doi.org/10.1582/LEUKOS.2006.03.01.001

Ricciardi, P., \& Buratti, C. (2018). Environmental quality of university classrooms: Subjective and objective evaluation of the thermal, acoustic, and lighting comfort conditions. Building and Environment, 127(September 2017), 23-36. https://doi.org/10.1016/j.buildenv.2017.10.030

Rindel, J. H. (2002). Acoustical comfort as a design criterion for dwellings in the future. 16th Biennial Conference of the New Zealand Acoustical Society: Sound in the Built Environment, (November), 1-9. Retrieved from https://www.researchgate.net/profile/Jens_Rindel/publication/276286140_Acoustical_comfort_as_a _design_criterion_for_dwellings_in_the_future/links/5555d71808ae980ca60b30f1/Acousticalcomfort-as-a-design-criterion-for-dwellings-in-the-future.pdf

Rockcastle, S., Amundadottir, M. L., \& Andersen, M. (2017). Contrast measures for predicting perceptual effects of daylight in architectural renderings. Lighting Research and Technology, 49(7), 882-903. https://doi.org/10.1177/1477153516644292

Rockcastle, S., \& Andersen, M. (2012). Dynamic Annual Metrics for Contrast in Daylit Architecture. In SimAUD 2012 - Symposium on Simulation for Architecture and Urban Design. Orlando, FL, USA.

Rockcastle, S., \& Andersen, M. (2013). Celebrating Contrast and Daylight Variability in Contemporary Architectural Design: A Typological Approach. Lux Europa, 17-19 September, 1-6. Retrieved from http://infoscience.epfl.ch/record/186396

Rockcastle, S., \& Andersen, M. (2014). Measuring the dynamics of contrast \& daylight variability in architecture: A proof-of-concept methodology. Building and Environment, 81, 320-333. https://doi.org/10.1016/j.buildenv.2014.06.012

Rodriguez, G., \& Siret, D. (2009). The future of houses: what real-estate ads tell about the evolution of singlefamily dwellings. International Journal of Architectural Research, 3(1), 92-100.

Roetzel, A., DeKay, M., Nakai Kidd, A., Klas, A., Sadick, A. M., Whittem, V., \& Zinkiewicz, L. (2020). Architectural, indoor environmental, personal and cultural influences on students' selection of a preferred place to study. Architectural Science Review, 63(3-4), 275-291. https://doi.org/10.1080/00038628.2019.1691971

Rohles, F. H. (2007). Temperature \& Temperament - A Psychologist Looks at Comfort. ASHRAE Journal, (February), 14-22.

Rothschild, M., \& Stiglitz, J. E. (1976). Equilibrium in Competitive Insurance Markets: An Essay on the Economics of Imperfect Information. The Quarterly Journal of Economics, 90(4), 629-649.

Roudsari, M. S., \& Pak, M. (2013). Ladybug: A parametric environmental plugin for grasshopper to help designers create an environmentally-conscious design. Proceedings of BS 2013: 13th Conference of the International Building Performance Simulation Association, 3128-3135.

Rowlands, T., Waddell, N., \& McKenna, B. (2015). Are we there yet? A technique to determine theoretical saturation. Journal of Computer Information Systems, 56(1), 40-47. https://doi.org/10.1080/08874417.2015.11645799

Sarbu, I., \& Sebarchievici, C. (2013). Aspects of indoor environmental quality assessment in buildings. Energy and Buildings, 60, 410-419. https://doi.org/10.1016/j.enbuild.2013.02.005

Sarey Khanie, M., Stoll, J., Einhäuser, W., Wienold, J., \& Andersen, M. (2017). Gaze and discomfort glare, Part 1: Development of a gaze-driven photometry. Lighting Research and Technology, 49(7), 845-865. https://doi.org/10.1177/1477153516649016

Sauerwein, E., Bailom, F., Matzler, K., \& Hinterhuber, H. H. (1996). The Kano model: How to delight your customers. In IX International Working Seminar on Production Economics (Vol. 1, pp. 313-327). Innsbruck, Austria.

Sawicki, D., \& Wolska, A. (2015). Discomfort glare prediction by different methods. Lighting Research and Technology, 47(6), 658-671. https://doi.org/10.1177/1477153515589773

Schiffman, H. R. (2000). Sensation and Perception: An Integrated Approach (Fifth Edit). John Wiley \& Sons.

Schwandt, T. A., Lincoln, Y. S., \& Guba, E. G. (2007). Judging interpretations: But is it rigorous? Trustworthiness and authenticity in naturalistic evaluation. New Directions for Evaluation, (114), 11-25. https://doi.org/https://doi.org/10.1002/ev.223

Schweiker, M., Ampatzi, E., Andargie, M. S., Andersen, R. K., Azar, E., Barthelmes, V. M., ... Zhang, S. (2020). Review of multi-domain approaches to indoor environmental perception and behaviour. Building and Environment, 106804. https://doi.org/10.1016/j.buildenv.2020.106804

Schweiker, M., André, M., Al-Atrash, F., Al-Khatri, H., Alprianti, R. R., Alsaad, H., ... Zomorodian, Z. S. (2020). Evaluating assumptions of scales for subjective assessment of thermal environments - Do 
laypersons perceive them the way, we researchers believe? Energy and Buildings, 211(March), 109761. https://doi.org/10.1016/j.enbuild.2020.109761

Schweiker, M., Fuchs, X., Becker, S., Shukuya, M., Dovjak, M., Hawighorst, M., \& Kolarik, J. (2017). Challenging the assumptions for thermal sensation scales. Building Research and Information, 45(5), 572589. https://doi.org/10.1080/09613218.2016.1183185

Schweiker, M., Rissetto, R., \& Wagner, A. (2020). Thermal expectation: Influencing factors and its effect on thermal perception. Energy and Buildings, 210. https://doi.org/10.1016/j.enbuild.2019.109729

Simon, H. A. (1955). A Behavioral Model of Rational Choice. The Quarterly Journal of Economics, 69(1), 99-118.

Smith, T. R., \& Clark, W. A. V. (1982). Housing market search behavior and expected utility theory: 1. Measuring preferences for housing. Environment and Planning A, 14, 681-698. https://doi.org/10.1068/a140681

Song, Y., Sun, Y., Luo, S., Tian, Z., Hou, J., Kim, J., ... de Dear, R. (2018). Residential adaptive comfort in a humid continental climate - Tianjin China. Energy and Buildings, 170, 115-121. https://doi.org/10.1016/j.enbuild.2018.03.083

Stanovich, K. E., \& West, R. F. (2000). Individual Differences in Reasoning: Implications for the Rationality Debate. Behavioral and Brain Sciences, 23, 645-726. https://doi.org/10.1017/S0140525X00003435

Steemers, K., \& Steane, M. A. (Eds.). (2004). Environmental Diversity in Architecture. Spon Press.

Stevenson, F. (2019). Embedding building performance evaluation in UK architectural practice and beyond. Building Research and Information, 47(3), 305-317. https://doi.org/10.1080/09613218.2018.1467542

Stiglitz, J. E. (2002). Information and the Change in the Paradigm in Economics. The American Economic Review, 92(3), 460-501.

Sundell, J. (2004). On the history of indoor air quality and health. Indoor Air, Supplement, 14(SUPPL. 7), 51-58. https://doi.org/10.1111/j.1600-0668.2004.00273.x

Tamas, R., O’Brien, W., \& Gunay, B. (2018). Summertime window use behaviour in air-conditioned condominiums, 502-511.

Telfar-Barnard, L., Bennett, J., Howden-Chapman, P., Jacobs, D. E., Ormandy, D., Cutler-Welsh, M., ... Keall, M. (2017). Measuring the effect of housing quality interventions: The case of the New Zealand "rental warrant of fitness." International Journal of Environmental Research and Public Health, 14(11). https://doi.org/10.3390/ijerph14111352

Thakkar, J. J. (2020). Structural Equation Modelling. Springer Nature Singapore Pte Ltd. https://doi.org/https://doi.org/10.1007/978-981-15-3793-6

Thaler, R. H. (1999). Mental accounting matters. Journal of Behavioral Decision Making, 12(3), 183-206.

Thaler, R. H. (2018a). Economia del comportamiento: pasado, presente y futuro. Revista de Economia Institucional, 20(38), 9-43.

Thaler, R. H. (2018b). From Cashews to Nudges: The Evolution of Behavioral Economics. American Economic Review, 108(6), 1265-1287. https://doi.org/10.1257/aer.108.6.1265

Thaler, R. H., \& Sunstein, C. R. (2008). Nudge: Improving decisions about health, wealth, and happiness.

Train, K. E. (2002). Discrete Choice Methods with Simulation. Econometric Reviews. Cambridge university press. https://doi.org/10.1080/07474938.2014.975634

Trocchia, P. J., Swanson, D. L., \& Orlitzky, M. (2007). Digging deeper: The laddering interview, a tool for surfacing values. Journal of Management Education, 31(5), 713-729. https://doi.org/10.1177/1052562906293611

Tversky, A., \& Kahneman, D. (1992). Advances in Prospect Theory: Cumulative Representation of Uncertainty. Journal of Risk and Uncertainty, 5, 297-323. https://doi.org/Doi 10.1007/Bf00122574

Tzempelikos, A., \& Athienitis, A. K. (2007). The impact of shading design and control on building cooling and lighting demand. Solar Energy, 81(3), 369-382. https://doi.org/10.1016/j.solener.2006.06.015

Tzempelikos, A., Athienitis, A. K., \& Karava, P. (2007). Simulation of façade and envelope design options for a new institutional building. Solar Energy, 81(9), 1088-1103. https://doi.org/10.1016/j.solener.2007.02.006

Tzempelikos, A., Bessoudo, M., Athienitis, A. K., \& Zmeureanu, R. (2010). Indoor thermal environmental conditions near glazed facades with shading devices - Part II: Thermal comfort simulation and impact of glazing and shading properties. Building and Environment, 45(11), 2517-2525.

https://doi.org/10.1016/j.buildenv.2010.05.014

U.S. Department of Energy. (2021). EnergyPlus website. 
Van Den Wymelenberg, K., \& Inanici, M. (2009). A study of luminance distribution patterns and occupant preference in daylit offices. PLEA 2009 - Architecture Energy and the Occupant's Perspective: Proceedings of the 26th International Conference on Passive and Low Energy Architecture.

van Hoof, J., Mazej, M., \& Hensen, J. L. M. (2010). Thermal comfort: research and practice. Frontiers in Bioscience, 15(2), 765-788. https://doi.org/10.2741/3645

Vardaxis, N.-G., \& Bard, D. (2018a). Review of acoustic comfort evaluation in dwellings: Part II-impact sound data associated with subjective responses in laboratory tests. Building Acoustics, 25(2), 171-192. https://doi.org/10.1177/1351010X18788685

Vardaxis, N.-G., \& Bard, D. (2018b). Review of acoustic comfort evaluation in dwellings: Part III-airborne sound data associated with subjective responses in laboratory tests. Building Acoustics, 25(4), 289-305. https://doi.org/10.1177/1351010X18788685

Vardaxis, N.-G., Bard, D., \& Persson Waye, K. (2018). Review of acoustic comfort evaluation in dwellingspart I: Associations of acoustic field data to subjective responses from building surveys. Building Acoustics, 25(2), 151-170. https://doi.org/10.1177/1351010x18762687

Veitch, J. A. (2001). Psychological processes influencing lighting quality. Journal of the Illuminating Engineering Society, 30(1), 124-140. https://doi.org/10.1080/00994480.2001.10748341

Veitch, J. A., \& Newsham, G. R. (2000). Exercised control, lighting choices, and energy use: An office simulation experiment. Journal of Environmental Psychology, 20(3), 219-237. https://doi.org/10.1006/jevp.1999.0169

Vera, S., Bustamante, W., Molina, G., \& Uribe, D. (2016). A flexible and time-efficient schedule-based communication tool for integrated lighting and thermal simulations of spaces with controlled artificial lighting and complex fenestration systems. Journal of Building Performance Simulation, 9(4), 382-396. https://doi.org/10.1080/19401493.2015.1062556

Villeneuve, H., \& O’Brien, W. (2020). Listen to the guests: Text-mining Airbnb reviews to explore indoor environmental quality. Building and Environment, 169. https://doi.org/10.1016/j.buildenv.2019.106555

Ward, G. (1994). The RADIANCE lighting simulation and rendering system. Proceedings of the 21st Annual Conference on Computer Graphics and Interactive Techniques - SIGGRAPH '94, 459-472. https://doi.org/10.1145/192161.192286

Ward, G., \& Shakespeare, R. A. (1998). Rendering With Radiance: The Art and Science of lighting visualization.

Wasilewski, S., Grobe, L. O., Wienold, J., \& Andersen, M. (2019). A Critical Literature Review of SpatioTemporal Simulation Methods for Daylight Glare Assessment. SDAR* Journal of Sustainable Design \& Applied Research, 7(1). https://doi.org/https://doi.org/10.21427/87r7-kn41

Wienold, J. (2009). Dynamic Daylight Glare Evaluation. In Eleventh International IBPS A Conference (pp. 944 951).

Wienold, J., \& Christoffersen, J. (2006). Evaluation methods and development of a new glare prediction model for daylight environments with the use of CCD cameras. Energy and Buildings, 38(7), 743-757. https://doi.org/10.1016/j.enbuild.2006.03.017

Wienold, J., Frontini, F., Herkel, S., \& Mende, S. (2011). Climate based simulation of different shading device systems for comfort and energy demand. In 12th Conference of International Building Performance Simulation Association (pp. 14-16). Sydney.

Wienold, J., Iwata, T., Sarey Khanie, M., Erell, E., Kraftan, E., Rodriguez RG, ... Andersen, M. (2019). Cross-validation and robustness of daylight glare metrics. Lighting Research \& Technology, 0, 1-31. https://doi.org/10.1177/1477153519826003

Wyer, R. S. (2008). The Role of Knowledge Accessibility in Cognition and Behavior. In C. P. Haugtvedt, P. M. Herr, \& F. R. Kardes (Eds.), Handbook of Consumer Psychology (pp. 31-76). New York, New York, USA: Taylor \& Francis.

Yan, D., \& Hong, T. (2018). International Energy Agency, EBC Annex 66 - Definition and Simulation of Occupant Behavior in Buildings.

Yao, R., Li, B., \& Liu, J. (2009). A theoretical adaptive model of thermal comfort - Adaptive Predicted Mean Vote (aPMV). Building and Environment, 44(10), 2089-2096. https://doi.org/10.1016/j.buildenv.2009.02.014

Zeithaml, V. A. (1988). Consumer perceptions of price, quality and value: a means-end model and synthesis of evidence. Journal of Marketing, 52(July), 2-22.

Zeitzer, J. M., Dijk, D. J., Kronauer, R., Brown, E., \& Czeisler, C. (2000). Sensitivity of the human circadian pacemaker to nocturnal light: melatonin phase resetting and suppression. The Journal of Physiology, $526 \mathrm{Pt}$ 3(Pt 3), 695-702. https://doi.org/10.1111/j.1469-7793.2000.00695.x 
Zhang, H. (2003). Human Thermal Sensation and Comfort in Transient and Non-Uniform Thermal Environments. University of California, Berkeley.

Zhang, H., Arens, E., Huizenga, C., \& Han, T. (2010a). Thermal sensation and comfort models for nonuniform and transient environments, part II: Local comfort of individual body parts. Building and Environment, 45(2), 389-398. https://doi.org/10.1016/j.buildenv.2009.06.018

Zhang, H., Arens, E., Huizenga, C., \& Han, T. (2010b). Thermal sensation and comfort models for nonuniform and transient environments, part III: Whole-body sensation and comfort. Building and Environment, 45(2), 399-410. https://doi.org/10.1016/j.buildenv.2009.06.020

Zhou, X., Ouyang, Q., Zhu, Y., Feng, C., \& Zhang, X. (2014). Experimental study of the influence of anticipated control on human thermal sensation and thermal comfort. Indoor Air, 24(2), 171-177. https://doi.org/10.1111/ina.12067 
11 APPENDICES 


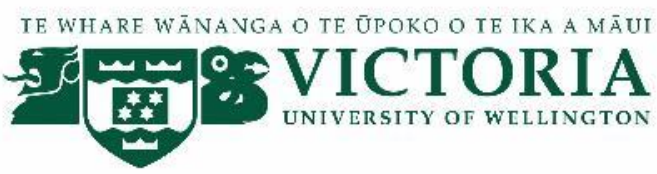

\title{
What do people expect from good dwellings?
}

\author{
Interview Guide \\ Student Researcher: German Molina (PhD student)
}

The purpose of this concise interview is to exploit people's experience in living within built environments in order to design new indoor environmental quality (IEQ) metrics. That is, to ask the respondents how they understand high IEQ within dwellings not only in physical terms (i.e., inputs to new IEQ metrics); but also, in terms of the benefits it affords to them (i.e., outputs to new IEQ metrics). It focuses on a single IEQ attribute at the time. However, since it is relatively short (about 20 minutes), a number of sequential interviews can be made in one session with the same respondent. It is not an in-depth interview.

In general, respondents will be asked to describe a dwelling with a particularly high quality of some IEQ attribute (i.e., "describe a warm dwelling"). Respondents will be interviewed one by one in a public place.

The main outcome of this interview is a concept structure relating housing attributes (physical or not). An example of such structure-obtained through a pilot interview-is found in Figure 1. Inspired on Coolen's Meaning Structure method (Coolen, 2008), such a structure is built during the interview with the respondent. The audio is recorded as backup, and used only in case of uncertainty. No transcription is required (Coolen, 2008).

The process is as follows:

\section{Introduction to convey the interviewee}

- Introduce myself, and thank them for their participation

- Remind them they are free to decline to reply to any question or can end the interview at any time.

- Handle them the information sheet and the informed consent for them to read, and ask them if they have any questions about the research.

- Explain the topic in general terms

- Outline what will happen to the data, especially remind them that all information is confidential and that any publications or reports will not involve any identifying aspects.

- Explain that the notes are meant to be taken by both people. Namely, the respondent is welcome and encouraged to actively participate and correct the interviewee if needed.

- Ask them for consent if they will participate in the research.

- Confirm that they are aware they can withdraw information at any time during data collection, before 31 of December, 2019. 


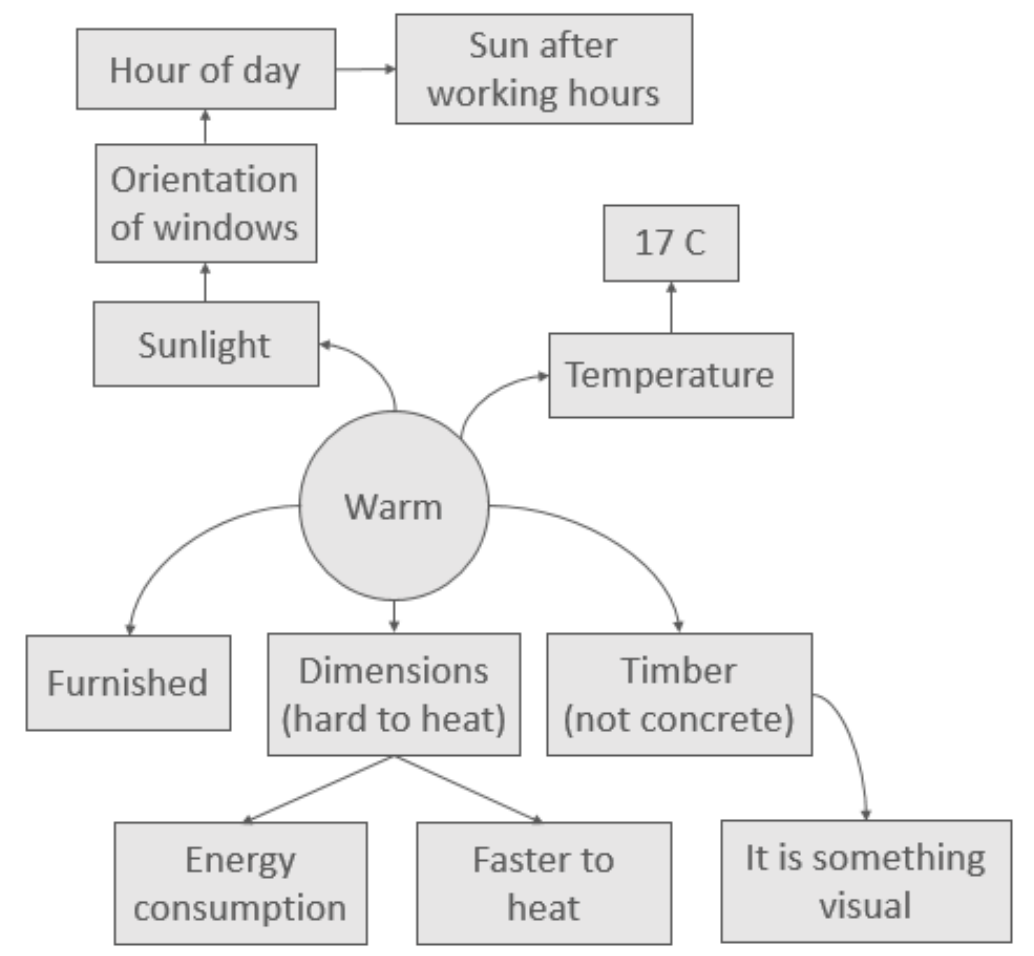

Results from the first test of the proposed interview method

\section{Background and warmup questions}

1. The date, time and location are recorded on tape and in the notes. This information is enough to match audio with notes.

2. How old are you?

3. What is your gender?

4. Who do you live with? (i.e., how many children and adults, what ages)

5. What is your marital status?

6. What is your occupation?

7. Do you own or rent your current home?

8. Are you currently searching for a home?

- If not,

i. When was the last time you did it?

ii. Was that the first time you did it?

- If yes,

i. Is this the first time you search for a new home?

9. What kind of home are/were you looking for? (e.g., apartment, house)

10. Where do you usually get information about available homes? (e.g., internet, newspaper, etc.)

11. How often do you get any information about warmness, natural light, humidity, ventilation and that kind of attributes?

12. Do you trust this information when it is available? Do you trust it more from some sources than others?

13. Would you like to have this information during your search, if it came from a trustworthy source? Are, these attributes, important? 


\section{Main questions}

Besides the general questions of the section above, this interview has only one main question:

"Please describe a warm dwelling. That dwelling does not need to exist. Take into account that you can answer this question in terms of features (i.e., size, materials, etc.) and on what does it afford to you (i.e., what is good about it when you wake up in the morning, or when you come back home from work, etc.)"

(Note that, instead of "warm", other domains might be asked.)

In the pilot interviews, this question was enough. However, there are several probe questions that would allow asking for the more specific information that this interview intends to extract. Namely:

1. How does the respondent understand high IEQ within dwellings in physical terms (i.e., inputs to new IEQ metrics)

a. How about the physical aspect... what are the materials of this dwelling? Is it big? What colours? Is it furnished?

b. When you go see a house, and you want it to be warm (or naturally illuminated), what do you search for?

2. How does the respondent understand high IEQ within dwellings with regards to the benefits it affords to them (i.e., outputs to new IEQ metrics)

a. What is better from a warm (or naturally illuminated) dwelling than from a cold (or dark) one?

b. How would you describe your life in a warm (or naturally illuminated) dwelling?

3. If something is unclear, vague or missing:

a. What do you mean by [term]?

b. Can [these block] be summarized as [a single word or term]?

c. Can you provide an example of [term]?

d. Why is this relevant for you?

e. Is this something you see in all dwellings you visit during your search?

\section{Finishing}

The structure is considered complete when the respondent considers that there is nothing more to add. After reaching such stage, the notes are reviewed and checked by the respondent and the interviewer, and the audio recording is stopped.

\section{Record}

The structure built along with the respondent (Figure 1), and all the annotations in it, are the raw data of this interview. This data has been already checked by the participant by the end of the data collection. 


\section{Questions translated into Spanish}

\section{$\underline{\text { Background and warmup questions }}$}

1. La fecha, hora y ubicación de la entrevista son registrados en las notas y en el audio. Esta información es suficiente para identificar qué notas corresponden a cada audio

2. ¿Cuántos años tienes?

3. ¿Cuál es tu género?

4. ¿Quién vive contigo?

5. ¿Cuál es tu estado civil?

6. ¿Cuál es tu ocupación?

7. ¿Arriendas o eres dueño de tu vivienda actual?

8. ¿Estás buscando una nueva vivienda en este momento?

- Si no,

i. ¿Cuándo fue la última vez que buscaste?

ii. ¿Fue esa la primera vez que buscaste?

- Si sí,

i. ¿Es esta la primera vez que buscas una nueva vivienda?

9. ¿Qué tipo de vivienda estas buscando? (casa, departamento)

10. ¿Dónde sueles buscar información respecto a viviendas disponibles? (internet, diarios, etc.)

11. ¿Qué tan seguido encuentras información respecto a la calidez, la iluminación natural o luminosidad, humedad, ventilación y ese tipo de características?

12. ¿Confías en esa información cuando está disponible?

13. ¿Te gustaría tener esa información durante tu búsqueda, si esta fuera ofrecida por alguna entidad confiable?

Main questions

"Por favor describa una vivienda calida. Esta vivienda no tiene por qué existir. Ten en cuenta que la descripción puede relacionarse con cosas físicas (e.g., materiales, tamaño, etc.) asi como también a tu calidad de vida (e.g., que ocurre cuando te levantas en la mañana?)

\section{Probe questions:}

1. How does the respondent understand high IEQ within dwellings in physical terms (i.e., inputs to new IEQ metrics)

a. ¿Y qué hay del aspecto físico? ¿Cuáles son los materiales de esta vivienda? ¿Es grande? ¿Qué colores predominan? ¿Esta amoblada?

b. Cuando vas a una vivienda y quieres que sea cálida (o naturalmente iluminada), ¿En qué te fijas?

2. How does the respondent understand high IEQ within dwellings with regards to the benefits it affords to them (i.e., outputs to new IEQ metrics) 
a. ¿Qué es mejor en una vivienda cálida (o naturalmente iluminada) que en una vivienda fría (u obscura)?

b. ¿Cómo describirías tu vida en una vivienda cálida (o naturalmente iluminada)?

3. If something is unclear, vague or missing:
a. ¿A qué te refieres con [término]?
b. ¿Puede [este bloque] resumirse como [una palabra o concepto]?
c. ¿Podrías darme un ejemplo de [término]?
d. ¿Por qué es esto relevante para ti?
e. ¿Es esto algo que ves en todas las viviendas que visitas durante tu búsqueda? 


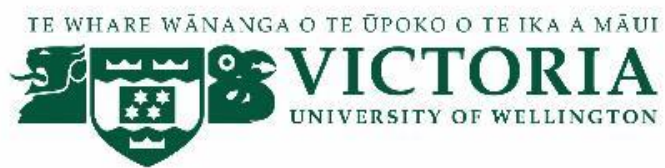

\section{What do people expect from good dwellings? INFORMATION SHEET FOR PARTICIPANTS}

You are invited to take part in this research. Please read this information before deciding whether or not to take part. If you decide to participate, thank you. If you decide not to participate, thank you for considering this request.

\section{Who am I?}

My name is Germán Molina and I am a Doctoral student in the School of Architecture at Victoria University of Wellington. This research project is work towards my dissertation.

\section{What is the aim of the project?}

This project explores consumers' understanding and expectation of warm and naturally illuminated dwellings.

This project was reviewed and approved by the Victoria University of Wellington Human Ethics Committee (ID 0000027329).

\section{How can you help?}

You have been invited to participate because I am interested in the opinion of people who are, or have been recently been, looking to purchase a new home. It is my understanding that you fit in this description.

If you agree to take part I will interview you at a public place, such as a café or one of the campuses of VUW. I will ask you questions about your personal expectations of warm and naturally illuminated residential spaces. There are no right or wrong answers. The interview will take around 40 or 50 minutes. I will audio record the interview with your permission. You can choose to not answer any question or stop the interview at any time, without giving a reason. You can withdraw from the study at any time before 31 of December 2019. If you withdraw, the information you provided will be destroyed or returned to you.

\section{What will happen to the information you give?}

The research is completely confidential. Your name will not be used in the study and any information that may be used to identify you (e.g., name, address, contact information, and other) will not be included in any analysis. This information is only kept in order to allow you to withdraw from the study (before 31 of December of 2019) and for us to contact you in case new doubts come up. The original notes taken during the interview will only be reviewed by the researcher (student) and supervisors and will be securely stored in a locked cabinet and password protected computer. Only myself (the student) and my supervisors will be able to see them. A transcriber might be used to 
create a written transcript of the audio recorded interview. However, the transcriber will work under an agreement to keep all information confidential. No other people, such as real estate agents or any person who may have recruit you, will have access to the original notes, audio or transcript (if made).

A de-identified (i.e., without the your name, address, contact information, or other traceable data) copy of the notes taken during the interview will be kept indefinitely. This copy will only include information that will allow other researchers analyse the data. That is, only gender, age, and household composition will be maintained. This deidentified copy may also be shared with other researchers, and will be made publicly available in online data repositories specifically built for the purpose of sharing data between researchers. De-identified results of the analysis, such as coded, reorganized, and cleaned versions of the notes, may also be published in the mentioned repository.

\section{What will the project produce?}

The findings produced by this research will only be used for academic purposes. This includes my $\mathrm{PhD}$ dissertation, journal articles, conference publications and/or presentations, and/or books. Your own name and personal details will be kept confidential and any information and/or opinions that may be traceable to you will not be reported in any of these publications or presentations, and will be deleted on the 31 of December 2019.

Transcripts (if the interview were transcribed) and the electronically recorded interviews will be securely stored for five years upon completion of the study before it will be destroyed.

If you would like to review a copy of the transcript (if it is transcribed) and a copy of the notes taken during the interview, please provide your contact details on the consent form. You can be reassured that the written summary will not contain any information that is traceable to you or any of the other participants. The transcript or electronic recording would not be made publicly available on the online data repositories.

\section{If you accept this invitation, what are your rights as a research participant?}

You do not have to accept this invitation if you don't want to. If you do decide to participate, you have the right to:

- choose not to answer any question;

- $\quad$ ask for the recorder to be turned off at any time during the interview;

- $\quad$ withdraw from the study at any time during the interview;

- $\quad$ ask any questions about the study at any time;

- $\quad$ read over and comment on a written summary of your interview (i.e., the notes taken);

- be able to read any reports of this research by emailing the researcher to request a copy. 
If you have any questions or problems, who can you contact?

STUDENT RESEARCHER:

\section{Germán Molina}

$\mathrm{PhD}$ Student

School of Architecture

Faculty of Architecture and Design

Victoria University of Wellington

PO Box 600 Wellington

6140 New Zealand

german.molinalarrain@vuw.ac.nz

\section{SUPERVISORS:}

\section{Dr Michael Donn}

Associate Processor

$\underline{\text { School of Architecture }}$

Faculty of Architecture and Design

Victoria University of Wellington

PO Box 600 Wellington

6140 New Zealand

+6444636221

michael.donn@vuw.ac.nz

\section{Dr Micael-Lee Johnstone}

Senior Lecturer

$\underline{\text { School of Marketing and International Business }}$

Victoria Business School

Victoria University of Wellington

PO Box 600 Wellington

$\underline{6140 \mathrm{New} \text { Zealand }}$

+6444636933

micael-lee.johnstone@vuw.ac.nz

\section{Dr Casimir MacGregor}

Senior Social Scientist

BRANZ

1222 Moonshine Road, RD1, Porirua

$5381 \mathrm{New}$ Zealand

$+6442381315$

casimir.macgregor@branz.co.nz

\section{Human Ethics Committee information}

If you have any concerns about the ethical conduct of the research you may contact the Victoria University HEC Convenor: Dr Judith Loveridge. Email hec@ vuw.ac.nz or telephone +64-4-463 6028 . 


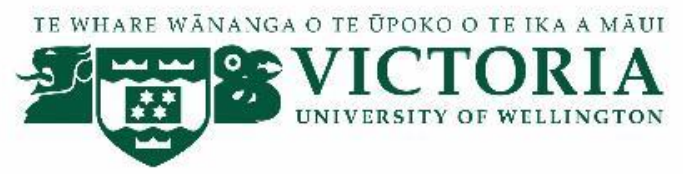

\section{¿Qué espera la gente de una buena vivienda? INFORMACIÓN PARA PARTICIPANTES}

Esta es una invitación para ser parte de mi investigación. Por favor lea este documento antes de decidir si participar o no. Si decide participar, muchas gracias. Si decide no hacerlo, muchas gracias por considerarlo.

\section{¿Quién soy?}

Mi nombre es Germán Molina Larrain y soy alumno de doctorado de la Escuela de Arquitectura de la Victoria University of Wellington. Esta investigación es parte de mi proyecto de doctorado, y conducirá a la elaboración de una tesis.

\section{¿Cuál es el objetivo de este proyecto?}

Este proyecto explora las expectativas y el entendimiento que los consumidores tienen respecto a viviendas cálidas y naturalmente iluminadas.

Este estudio ha sido revisado y aprobado por el Comité de Ética de Victoria University of Wellington (ID 0000027329).

\section{¿Cómo me puedes ayudar?}

Has recibido esta invitación porque me estoy interesado en la opinión de personas que se encuentran buscando comprar una vivienda, o lo han hecho durante los últimos 36 meses. Entiendo que tu cumples con estos requisitos.

Si decides participar, me gustaría entrevistarte en algún lugar público, como un café. Las preguntas estarán relacionadas a tu opinión y tus expectativas respecto a viviendas cálidas y naturalmente iluminadas. No hay respuestas incorrectas.

La entrevista debiese durar alrededor de 40 o 50 minutos. Ésta será grabada en audio, siempre y cuando accedas a ello. Puedes retirarte de este estudio en cualquier momento antes del 31 de diciembre de 2019, en cuyo caso toda la información provista será destruida o devuelta a ti.

\section{¿Qué ocurrirá con la información que yo entregue?}

Este estudio es completamente confidencial. Tu nombre no será utilizado y cualquier información que pueda ser usada para identificarte (e.g., nombre, dirección, información de contacto, u otro) no será incluida en ningún análisis. Esta información sólo se mantiene con el fin de permitir que te retires del estudio (antes del 31 de Diciembre de 2019) y para nosotros poder contactarte en caso de dudas. Las notas tomadas serán revisadas por el investigador (estudiante) y los supervisores, y será guardada de manera segura en un archivador bajo llave y en un computador con clave. Solamente yo (el investigador) y mis supervisores tendrán acceso a esta información. Una persona podría ser contratada para transcribir el audio en texto. Sin embargo, dicha persona trabajaría bajo un acuerdo de confidencialidad. Ninguna otra persona tendrá accesso a la información (como por ejemplo, corredores de propiedades o quien quiera que te haya reclutado para este estudio). 
Una copia no rastreable (es decir, sin tu nombre, dirección, datos de contacto u otra información que pueda identificarte) de las notas tomadas durante la entrevista será guardada de manera indefinida. Esta copia sólo incluirá la información necesaria para que otros investigadores puedan analizar los datos. Específicamente, solo el genero, edad, y composición del grupo familiar (quienes viven en la casa) serán mantenidos. Esta copia no rastreable puede ser compartida con otros investigadores y académicos, y será publicada en internét a través de un repositorio en línea diseñado para que investigadores compartan información. Resultados no rastreables del análisis de las notas tomadas, tales como versiones codificadas, limpias y reorganizadas, podrían ser publicadas en este repositorio también.

\section{¿Qué producirá este proyecto?}

Los resultados de esta investigación serán utilizados con propósitos académicos. Esto incluye mi tesis de doctorado, artículos científicos, presentaciones y/o publicaciones en conferencias, y libros. Tu nombre será confidencial y cualquier otro dato que pueda ser utilizado para identificarte no será utilizado en estos reportes.

Transcripciones (si es que el audio fuera transcrito) y el audio de esta investigación será guardado de manera segura hasta 5 años luego de completada la investigación. Luego de eso será destruida.

Si te interesa revisar la transcripción de tu entrevista (en caso de que se transcriba) y una copia de las notas tomadas durante la entrevista, por favor escribe tu dirección de correo electrónico en el formulario de consentimiento para esta entrevista. Puedes estar seguro que dicho reporte no incluirá ninguna información que pueda ser usada para identificarte a ti o a otros participantes. Ni la transcripción de la entrevista ni el audio serán publicados en internet por medio del mencionado repositorio de datos.

\section{Si acepto participar, ¿Cuáles son mis derechos como participante?}

No tienes la obligación de aceptar esta invitación si no quieres. Si decides aceptarlas, tienes el derecho a:

- No responder ninguna pregunta;

- Pedir que la grabadora sea apagada en cualquier momento durante la entrevista;

- Retirarme de la entrevista en cualquier momento;

- Hacer preguntas respecto al estudio en cualquier momento;

- Leer y opinar respecto al resumen de la entrevista (i.e., las notas tomadas);

- Leer cualquier reporte de esta investigación, por medio de una petición via e-mail al investigador 


\section{Si tengo alguna duda o problema ¿̇a quién puedo contactar?}

\section{ESTUDIANTE INVESTIGADOR:}

\section{Germán Molina}

$\mathrm{PhD}$ Student

Escuela de Arquitectura

Facultad de Arquitectura y Diseño

Victoria University of Wellington

PO Box 600 Wellington

$6140 \mathrm{New}$ Zealand

german.molinalarrain@vuw.ac.nz

\section{SUPERVISORES:}

\section{Dr Michael Donn}

Associate Processor

Escuela de Arquitectura

Facultad de Arquitectura y Diseño

Victoria University of Wellington

PO Box 600 Wellington

6140 New Zealand

+6444636221

michael.donn@vuw.ac.nz

\section{Dr Micael-Lee Johnstone}

Senior Lecturer

Escuela de Marketing y Negocios Internacionales

Escuela de Negocios de Victoria

Victoria University of Wellington

PO Box 600 Wellington

$6140 \mathrm{New}$ Zealand

+6444636933

micael-lee.johnstone@vuw.ac.nz

\section{Dr Casimir MacGregor}

$\underline{\text { Senior Social Scientist }}$

BRANZ

1222 Moonshine Road, RD1, Porirua

$5381 \mathrm{New}$ Zealand

$+6442381315$

casimir.macgregor@branz.co.nz

Información sobre el Comité de Etica de Victoria University of Wellington

Si tienes alguna duda respecto a la ética de esta investigación puedes contactar al comité de ética de Victoria Univesity of Wellington: Dr Judith Loveridge. Email hec@vuw.ac.nz o llamar al +64-4-463 6028. 


\section{What do people expect from good dwellings? CONSENT TO INTERVIEW}

This consent form will be held for 5 years.

Researcher: Germán Molina, Victoria University of Wellington.

- I have read the Information Sheet and the project has been explained to me. My questions have been answered to my satisfaction. I understand that I can ask further questions at any time.

- I agree to take part in an audio recorded interview.

I understand that:

- I may withdraw from this study at any point before 31 of December 2019, and any information that I have provided will be returned to me or destroyed.

- $\quad$ All the identifiable information I have provided will be destroyed on 31 of December 2019.

- The notes taken during this interview, without my name or traceable information attached, will be made publicly available in online data repositories, and may be shared directly with other researchers on request

- I may review the notes taken during the interview, and I may ask for the final version that will be made publicly available

- Any information I provide that may be used to identify me will be kept confidential to the researcher and the supervisor and potentially a transcriber.

- The results will be used for a PhD dissertation and/or academic publications, presentations, and/or books.

- My name will not be used in reports, nor will any information that would identify me.

I would like to review my interview transcript (if it is transcribed), a summary of the final report, and a copy of the notes taken during my interview, and I have added my email address below.

Signature of participant:

Name of participant:

Date:

Contact details: 


\section{¿Qué espera la gente de una buena vivienda? CONSENTIMIENTO PARA ENTREVISTA}

Este formulario de consentimiento es válido por 5 años.

Investigador: Germán Molina, Victoria University of Wellington.

- $\quad$ He leído este formulario de consentimiento y el proyecto se me ha explicado. Mis preguntas han sido satisfactoriamente contestadas. Entiendo que puedo hacer más preguntas en cualquier momento.

- Estoy de acuerdo en participar en una entrevista grabada en audio.

\section{Entiendo que:}

- Me puedo retirar de este estudio en cualquier momento antes del 31 de diciembre de 2019, y cualquier información que yo haya entregado será destruida.

- Toda la información que yo entregue y que pueda utilizarse para identificarme será destruida el 31 de diciembre de 2019.

- Las notas tomadas durante esta investigación, excluyendo mi nombre u otra información que pueda conectarse conmigo, será publicada en repositorios públicos de datos de investigación y puede ser compartida con otros investigadores si fuera requerida.

- $\quad$ Puedo reviser las notas tomadas durante la entrevista, y puedo pedir la versión final que será hecha pública.

- Cualquier información que yo entregue y que pueda ser utilizada para identificarme será mantenida de manera confidencial entre el investigador (yo), los supervisores de esta investigación, y potencialmente un transcriptor.

- Los resultados de esta investigación se utilizarán para desarrollar una tesis de doctorado y/o en publicaciones académicas, presentaciones, y/o libros.

- Mi nombre no se utilizará en los reportes, ni tampoco ninguna otra información que pueda utilizarse para identificarme.

Me gustaría recibir un resumen del reporte final, por lo que he incluido Yes Yes $\square \quad$ No mi dirección de correo electrónico al final de la página.

Firma del participante:

Nombre del participante:

Fecha:

Correo electrónico: 


\section{Description of the elements in Tables 3 to 16}

This appendix contains the elements contained in Tables 3 to 16 of this thesis in alphabetical order. That is to say, the elements identified as relevant in the determination of people's Feeling of Comfort. Each of these elements has also been position within the Atlas of Comfort, available in the link below. Following the link will not only show the description of the code but the quotes that are associated with it.

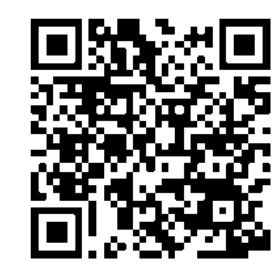

http://www.buildingsforpeople.org/atlas.html

\section{ACOUSTIC ABSORPTION}

Objective Indoor Climatic Factor related to the domain of Acoustics

A lack of Acoustic Absorption can produce an unpleasant experience in dwellings. It can affect the clarity of the audio thus making it difficult to hold conversations.

\section{ACOUSTIC INSULATION}

Environmental Cue related to the domain of Acoustics

Not all walls, windows or doors are equally effective at stopping the noise. People know this and therefore it becomes a relevant input to their assessment of a space.

Acoustic insulation is not only expected to stop outside noises but also noise transferred between spaces.

\section{ACOUSTIC PRIVACY}

Perception related to the domain of Acoustics

Privacy is the perception of being invaded by other people. Specifically for this model, it relates to other people hearing our actions.

This is different from the perception of Bothering Others because privacy is not about others being affected, but ourselves.

The Loss of Visual Privacy associated with Daylight and Coolness is the same concept. However, that case relates to the perception of being observed (e.g. through openings and windows).

\section{AESTHETICS}

Trade-off related to the domains of warmness, coolness and air quality

Some systems, materials, appliances, and design choices can be beneficial for the performance of the house while not being aesthetically attractive. This Trade-off, then, makes people choose between having a more comfortable but less beautiful home or having a more beautiful and less comfortable one. 


\section{AFFECTIVE ATTRIBUTES}

Environmental Cue related to the domains of warmness and coolness

When describing Warm and pleasantly cold (i.e., Cool) dwellings, respondents (predominantly those from Chile) mentioned attributes that seem to be more related to their aspirations and lifestyle than to any truly thermal factor. Specifically, elements that seem more related to how welcoming and liveable a dwelling is.

\section{AIR LEAKAGE}

Objective Indoor Climatic Factor related to the domain of Warmness

Air that leaks into homes (through windows, doors, and other elements) can affect people's experience in them. For instance, when a home is kept at a pleasant temperature, hot or cold air that leaks into the dwelling can drastically change its temperature. Moreover, being directly exposed to leaking air can produce unpleasant and dramatic changes in thermal sensation.

\section{AIR QUALITY VS ACOUSTIC PERFORMANCE}

Trade-Off related to the domains of Air Quality and Acoustics

Opening windows allows cleaning a dwelling's air. However, because of the existence of external noise, people sometimes need to choose between tolerating an unpleasant acoustic situation (i.e., by opening windows) and breathing what feels like unhealthy or polluted air (i.e., by keeping the windows closed). Moreover, even if ventilation systems can potentially solve this problem, they can also be noisy or can transmit noise, ultimately offering a similar set of alternatives: to tolerate noise or to tolerate air that is not clean.

\section{AIR TEMPERATURE}

Objective Indoor Climatic Factor related to the domains of Warmness, Coolness and Air Quality

The temperature of the air within a space is an important determinant of thermal comfort. This has been evidenced by the thermal comfort literature numerous times. However, the temperature of the air also seems to influence people's perception of air quality; specifically, low temperatures tend to be associated a cleaner air and high ones with less clean air.

It is possible that when people talk about "air temperature" they are actually talking about Operative Temperature or Mean Radiant Temperature without knowing it.

\section{ATTRACTIVENESS OF THE SPACE}

Perception related to the domain of Daylight

One of the functions of daylight, according to the respondents, is to make spaces more attractive. They look better and more welcoming.

\section{AVAILABILITY OF WINDOWS AND SKYLIGHTS}

Environmental Cue related to the domains of Daylight, Air Quality and Coolness

Windows and skylights are probably the most common and intuitive way of letting daylight and air into the dwelling. However, not all windows and skylights are equally effective. On the contrary, if a room has windows or skylights that are obstructed, or too small, or there are very few of them, people might not get the impression of it being properly illuminated.

As to what do people mean by "decent size" and "lots of windows", it is unknown. It is possible for this to depend on the views to the exterior, external obstructions, and some other factors. 


\section{AWARENESS OF AIR POLLUTION}

Environmental Cue related to the domain of Air Quality

Air pollution is not always visible and people are not always capable of perceiving it inside their homes. However, either because of the news or by looking at it from afar, people are often aware that pollution is there. This can psychologically affect their perception of air quality.

\section{BED TEMPERATURE}

Objective Indoor Climatic Factor related to the domain of Warmness

Sleeping is one of the things people do at home. They want to sleep in a bed with an appropriate temperature. If it is cold, it seems to be something they do remember.

The temperature of the bed is, obviously, related to the temperature of the bedroom. However, when talking about Warmness, people specifically mentioned the bed; whereas, when talking about coolness, they talked about the temperature of the whole room. This might be explained by the fact that, when it is too hot, people do not get $*^{*}{ }^{*}$ the bed, necessarily.

\section{BOTHERING OTHERS}

Perception related to the domains of Air Quality and Acoustics

The Feeling of Comfort is a state of the mind, and thus it is not limited to physiological sensations or even to a person's own body. One example of this is how people worry about bothering others.

For instance, people often worry about making noise that can be unpleasant for other people in the house and also of neighbours. Something similar might happen when cooking, in which the odours of one individual's kitchen can affect neighbours.

\section{BREEZES}

Objective Indoor Climatic Factor related to the domains of Coolness and Air Quality

Letting outside air come into the dwelling allows both reducing people's thermal sensation and cleaning the air. An actual breeze-as opposed to just heat-driven convective currents-seems to be the feedback that people need to perceive that fresh air is coming in.

\section{BUDGET}

Internal Element related to the domain of Warmness

While people do want to be comfortable, they will not necessarily spend all the money necessary to make their homes comfortable. On the contrary, some of them seem to have a pre-allocated budget for utility bills. When that budget is not enough, they might choose to sacrifice comfort in favour of keeping the utility bills low.

While a person's socioeconomic condition is likely to influence their budget, it is not necessarily true that people who can afford to do it will pay whatever it takes.

\section{BUGS}

Trade-off related to the domain of Coolness

Opening windows is very useful for cooling down dwellings. However, if there are bugs outside, this becomes an issue. Simple solutions, such as fly-screens, might be enough to solve this issue. 


\section{Cleanability OF THE SPACE}

Environmental Cue related to the domain of Air Quality

All homes will contain some dust and dirt. Nonetheless, not all of them are equally easy to clean. Materials that are easier to clean tend to be associated with better air quality. See also Dust and Smoke as an environmental cue.

\section{Cleanliness of THE SPACE}

Perception related to the domain of Daylight, Air Quality and Coolness

People care about how clean the spaces they use are. This perception of cleanliness seems to be influenced by how well illuminated the space is, by the perceived quality of its air, and by its temperature. Hot, humid and dark places are perceived as unhygienic.

\section{COLOUR OF THE SPACE}

Environmental Cue related to the domains of Daylight, Coolness and Air Quality

Daylight will penetrate more or less into the space depending on the colour (or, more specifically, the reflectivity) of the materials in the space. Lighter colours would allow daylight to get further into rooms, which was raised by some respondents.

Also, dark colours seem to be associated with cold and unhygienic spaces.

\section{COMPLETE SILENCE VS SOUNDS OF NATURE}

Trade-off related to the domain of Acoustics

People do not necessarily want absolute silence but for the soothing sound of nature (see code The Sound of Silence). However, sometimes they need to choose between blocking both the pleasant natural sounds as well as human noises, and letting both in. It would seem that people generally prefer to block it all.

\section{CONDENSATION AND MOULD}

Environmental Cue related to the domains of Warmness and Air Quality

Perceivable condensation and mould are indicators of cold and humidity, thus related to a lack of air quality and warmness.

\section{COOLNESS VS WARMNESS}

Trade-off related to the domains of Coolness and Warmness

This model treats coolness and warmness separately, but they are tightly related. People know that favouring one over the other might not be beneficial in the end. Balance is needed.

\section{DAMAGE TO OBJECTS IN THE HOUSE}

Trade-off related to the domain of Daylight

People like spaces with plenty of natural light. However, they know that solar radiation can damage objects in the dwelling.

\section{DAYLIGHT SUFFICIENCY}

Perception related to the domains of Daylight, Warmness, Coolness and Air Quality

A space will be perceived as "sufficiently illuminated" when it has just enough natural light for the sun to warm it up and operating without the need for electric lighting. However, this quantity does not seem to be nearly enough. People mentioned the need for quality of light instead of quantity of it and also that they want much more light than what is required to read a book.

Having enough daylight also seems to be a requisite for a dwelling to be described as Cool, and can make spaces feel cleaner thus favouring their Air Quality. 


\section{DAYLIGHT VS THERMAL COMFORT}

Trade-off related to the domains of Daylight and Coolness

Increasing windows to maximize daylight has the consequence of reducing thermal insulation and thus increasing the chances of feeling cold.

Also, maximizing daylight and warmth can lead to an excessively hot home in summer, if not done carefully. People need to choose, then, between having plenty of daylight while suffering from high temperatures and being thermally comfortable in a relatively dark home.

\section{DIRECT VS DIFFUSE LIGHT}

Objective Indoor Climatic Factor related to the domain of Daylight

Daylight can be separated into two components: diffuse and direct.

Examples of Diffuse Daylight are the light coming from the sky on a cloudy day and the light reflected by buildings or snow. This component of natural light creates gentle shadows and will often be less powerful than its direct component.

The most obvious example of Direct Daylight is the light that the sun casts on a clear sunny day. It is a powerful stream of light that creates strong and well-defined shadows.

Depending on the situation, people might prefer one over the other. Direct daylight carries with it a lot of heat, and thus it might be unpleasant for people who live in hot and sunny locations.

\section{DIRECTION OF THE LIGHT}

Objective Indoor Climatic Factor related to the domain of Daylight

While it is not entirely clear why this happens, the angle of the natural light entering the space might matter. Specifically, respondents from Santiago (Chile) argued that the more vertical the angle of natural light, the better. Two reasons for this were given. The first one is that vertical sun is easier to control (i.e. it does not strike over the whole room, just the perimeter), which is convenient for hot locations with strong solar radiation. The second reason was related to the fact that light coming from above emulates an exterior light setting better than lateral light.

\section{DOORS BETWEEN SPACES}

Environmental Cue related to the domains of Air Quality and Acoustic

Preventing noise and transmission of odours is not always easy. However, sometimes, having some doors that separate the different spaces in the dwelling is enough. This mechanism seems to be a good indicator of how noisy the dwelling might become in the future (i.e., it is relevant for expected outcomes).

\section{DUST AND SMOKE}

Environmental Cue related to the domain of Air Quality

While not always included in the list of relevant factors related to air quality, dust can be quite annoying for people. Different sites and neighbourhoods will be more or less exposed to dust. This suggests that the quality of the air will not be good.

Smoke, of course, has a similar effect. However, it can be less dependent on the site and the neighbourhood and more on neighbours. 


\section{DWELLING TYPOLOGY}

Internal Element related to the domains of Warmness and Acoustics

Depending on whether people are looking for, or live in, an apartment, detached or attached house, or a different kind of dwelling, their experiences and concerns will vary. This is particularly important for the long-term inferences people make when searching for a new home to live in.

\section{EFFECTIVE AND SIMPLE SYSTEMS}

Criterion for inferring Expected Outcomes related to the domains of Warmness, Air Quality, Acoustics and Coolness

Dwellings have systems that aim to make them more comfortable, such as heaters, mechanical ventilation, and electric lighting. In order to be utilized, the systems should act quickly and be simple to use. If the systems in a dwelling are ineffective at solving problems, people can feel helpless.

\section{ENERGY EFFICIENCY}

Trade-off related to the domain of Warmness

Heating, cooling, lighting and ventilating all consume energy. Some people expressed their concern with regards to the environmental impact of their actions. People will need to choose whether they want to be comfortable while producing some negative environmental impacts; or feeling relatively uncomfortable while lowering the environmental impacts of their actions.

While strongly related to the Trade-Off of Utility Bills, the motivation of favouring energy efficiency does not necessarily rely on financial estimations.

\section{EXPOSURE TO SOLAR RADIATION}

Objective Indoor Climatic Factor related to the domains of Warmness, Coolness and Air Quality

Even when the air in a room is maintained at a subjectively pleasant temperature, being exposed to solar radiation can significantly affect people's thermal perception and thermal comfort. If it is cold, the sun might be welcomed; but if it is hot, it is definitely not.

Also, rooms that are exposed to solar radiation are associated to a lack of humidity and dampness.

\section{EXPOSURE TO WIND}

Trade-Off related to the domain of Air Quality

In some places, wind can be strong enough to become an impediment to open windows. This can stop people from ventilating their homes and, in consequence, from enjoying clean air.

\section{FEELING OF CONFINEMENT}

Perception related to the domains of Daylight, Air Quality and Coolness

Some people reported that naturally illuminated spaces feel less enclosed. It is possible that this perception is related to the perception of Sense of Connection With the Exterior, although further research is needed to verify this. Similarly, it is hard to know whether this effect would still exist if daylight was provided without views.

The Feeling of Confinement is also perceived in spaces that are excessively hot or in which the air feels heavy and stuffy. As mentioned in the code of Air Temperature, these two variables - the perceived quality of the air and the air temperature-seem to be related. 


\section{FLOOR CONTACT TEMPERATURE}

Objective Indoor Climatic Factor related to the domain of Warmness and Coolness

The floor contact temperature seems to be an important determinant of the perception of thermal comfort in dwellings. This is affected by both the temperature of the floor top surface (as measured through a thermometer) and the thermal properties of the materials in the floor. Specifically, the material's thermal effusivity.

\section{FLOOR NUMBER}

Environmental Cue related to the domain of Coolness

Upper storeys or floors seem to be associated with more heat. Sometimes the explanation is that heat goes up (e.g., the upper floors in a house are hotter than the lower ones), and also that apartments that are higher take advantage of the heat of apartments below.

Some other explanations were identified (e.g., there are no windows or cooling) but, generally, it seems that upper storeys are warmer/hotter than others.

\section{FREEDOM TO DO WHAT YOU WANT WHEREVER YOU WANT}

A manifestation of Comfort related to the domains of Daylight, Warmness, Coolness, Air Quality and Acoustics

A comfortable home should allow people to carry on with their lives without being an impediment. People seem to want a home that will not significantly affect their lifestyles.

\section{GLARE}

Perception related to the domain of Daylight

Glare is the difficulty for seeing, strongly associated to excessive contrast or brightness. While quite important in office settings, it seems to be relatively irrelevant in residential settings.

\section{HEATING AND COOLING SYSTEM}

Environmental Cue related to the domains of Warmness and Coolness

In areas that are too cold or hot, having a mechanical heating and/or cooling system might be the only option for ensuring that the space can be maintained at a pleasant temperature.

\section{HOUSEHOLD COMPOSITION}

Internal Element related to the domains of Warmness, Coolness, Air Quality and Acoustics

The Feeling of Comfort is a state of the mind, and thus it is not limited to physiological sensations or even to individuals' own body. The health and wellbeing of others, such as children and family, can deeply affect a person's satisfaction with a home, thus affecting their mental wellbeing.

\section{INCREASED MAINTENANCE AND REDUCED DURABILITY}

Trade-Off related to the domains of Daylight, Air Quality and Coolness

Some technologies and construction materials are beneficial for comfort. However, they sometimes carry with them extra costs or do not last as much as other alternatives.

\section{LIFESTYLE}

Internal Element related to the domain of Warmness, Coolness, Air Quality and Acoustics

An important component of the Feeling of Comfort is the freedom to do what you want wherever you want (see code with that name). In other words, people want homes that do not prevent them from living according to their desired lifestyle. 


\section{LIGHT POLLUTION}

Trade-Off related to the domain of Coolness

Opening curtains and windows at night can become an issue when there is too much light outside. This can prevent people from cooling down their homes by ventilating them during the night.

\section{LOCATION}

Trade-Off related to the domain of Acoustics

Location is one of the first factors that people consider when searching for a new home. Commuting is, generally, not fun at all. However, while making this choice, people need to decide between enjoying the benefits of a centrally located home or the quietness and peace of the more remote suburbs.

Of course, the streets nearby will also influence this trade-off. For instance, there might be some centrally located homes that have a good acoustic performance due to the specific street they are located in. However, this is not necessarily the case for all homes.

\section{LOSS OF VIEWS}

Trade-Off related to the domains of Daylight, Acoustics and Coolness

Views are not always in the direction that maximizes daylight, minimizes noise, and ensures coolness. Thus, people sometimes need to choose between enjoying Views and living in a Cool dwelling with good Acoustic Performance and Daylight.

\section{LOSS OF PRIVACY}

Trade-Off related to the domains of Daylight and Coolness

Increasing the transparency in the facade increases the amount of natural light, but it also reduces visual privacy. Similarly, choosing the right orientation and opening windows often allows preventing overheating in the dwelling. However, these two actions will sometimes lead to facing other apartments and/or to being visible from the outside.

\section{LOUDNESS VS ORIGIN}

\section{Perception related to the domain of Acoustics}

Loudness is a measure of how strong a sound is, and depends mostly on pressure level (i.e. decibels) and frequency. However, it is important to acknowledge that noises (i.e., unwanted sounds) of equal loudness may be more or less unpleasant at different times of the day or in different situations and depending on their source.

People distinguish clearly between different sources of noise.

- Noise from neighbours

- Noise from common spaces (e.g. pools, parks, lobbies)

- $\quad$ City noise (e.g. traffic, horns, etc.)

- Toilet and pipes noise

- Noises that come from the house itself (e.g. squeaky doors, cracking floors)

- Noises produced by those who live in the house (e.g. kitchen appliances, children, pets)

These different sources will trigger different cognitive responses. For instance, noises from neighbours, common spaces, and those from the city cannot be controlled and are not part of the natural consequence of their actions, thus being highly unpleasant. On the contrary, the noise produced by those who live in the house is relatively easier to control and is, indeed, a natural consequence of their actions, therefore less unpleasant. 
Toilet and pipes noises, on their part, are likely to contribute to a perception of reduced Acoustic Privacy. Finally, noises that come from the house itself are interpreted as an indicator of wear and tear, and therefore are unpleasant.

\section{LOVE FOR MUSIC}

Internal Element related to the domain of Acoustics

People who enjoy listening to or playing music may be particularly sensitive to acoustic conditions. They might worry about bothering others with their music, meaning that the dwelling is stopping them from freely choosing their lifestyle.

\section{MATERIALS PERCEIVED AS HUMID}

Environmental Cue related to the domain of Air Quality

There seem to be some construction materials that, according to people, absorb water and thus tend to be more or less beneficial to air quality. Nonetheless, only a few people mentioned this and they do not agree with each other.

\section{MATERIALS THAT FEEL WARM}

Environmental Cue related to the domains of Warmness and Coolness

People seem to perceive some materials as being warmer than others. This might be related to their thermal effusivity-mentioned also when discussing the Floor contact temperature-but also to more aesthetic reasons. Further research is required.

\section{MEAN RADIANT TEMPERATURE}

Objective Indoor Climatic Factor related to the domain of Coolness

Sometimes it is not the air that is hot or the sun that is striking but heat irradiated from certain hot surfaces. It feels different.

\section{MENTAL WELLBEING}

A manifestation of Comfort related to the domains of Daylight, Warmness, Coolness, Air Quality and Acoustics

A comfortable home makes people happy and fills them with energy. It allows them to be sure that their families will be healthy.

\section{NEIGHBOURHOOD CHARACTERISTICS}

\section{Environmental Cue related to the domains of Air Quality and Acoustics}

The infrastructure and the nature of the constructions in different neighbourhoods affect the way these are perceived by people. Some neighbourhoods, for example, have a great number of restaurants, others are close to land fields, and others only have offices and thus are nearly empty at night.

Similarly, institutional differences can also affect the way in which neighbourhoods perform. For instance, some neighbourhoods allow people to use log burners and/or pubs to stay open till late while others do not. This affects the quality of life of the people who live in it.

This code, together with Neighbourhood demographics, describe the location at a neighbourhood scale. Specifically, while Demographics relates to the human factors (e.g., people are quiet, old, young, etc.), this code describes the more physical factors (e.g., neighbouring infrastructure, restaurants, etc.). Also, the code of Site characteristics describes the location at a smaller scale. 


\section{NEIGHBOURHOOD DEMOGRAPHICS}

Environmental Cue related to the domain of Acoustics

Not everyone likes to do the same things at the same time. These differences can be, at least in part, explained by the demographics of different groups. For instance, young people tend to stay until late while older people or families tend to get up early. This affects the experience of people in certain neighbourhoods.

This code, together with Neighbourhood characteristics, describe the location at a neighbourhood scale. Specifically, while Demographics describe the human factors, the Characteristics describe the more physical factors (e.g., neighbouring infrastructure, restaurants, etc.)

\section{NUISANCES AND LACK OF SAFETY}

Trade-Off related to the domains of Warmness, Coolness and Air Quality

Just as in the case of Utility Bills, safety can be a priority for people. Thus, all appliances and systems in their homes should be safe to operate so people are not forced to choose between their safety and their comfort. Also, operating these systems should not be unpleasant enough for them to be left unused.

\section{ODOURS}

Perception related to the domain of Air Quality

The sense of smell is used to assess air quality. Bad smells are associated with poor air quality.

\section{OPENNESS OF THE SPACE}

Trade-Off related to the domain of Acoustics

Having more subdivisions between spaces (e.g., doors) reduces the transmission of noise and odours across the space. However, some people would rather not have these doors due to their lifestyle and preferences. This is a choice they have to make.

\section{ORIENTATION}

Environmental cue related to the domains of Daylight, Warmness and Coolness

The sun does not strike equally in all directions. In the northern hemisphere, the sun will generally strike from the South; while in the southern hemisphere, from the North. These differences imply that some orientations will receive more sunlight and, therefore, be hotter, brighter and more or less pleasant.

\section{PASSIVE DESIGN}

Criterion for inferring Expected Outcomes related to the domains of Daylight, Warmness, Coolness, Air Quality and Acoustics

Having heating, lighting, cooling, and ventilation systems that are simple to use, effective, cheap to run, and reliable is a good thing. However, having a home that does not often rely on these systems is even better.

\section{PETS}

Environmental Cue related to the domains of Acoustics and Air Quality

Pets-specifically neighbours' pets_can be the source of noise, allergies, and other nuisances. 


\section{PHYSICAL WELLBEING}

A manifestation of Comfort related to the domains of Daylight, Warmness, Coolness, and Air Quality

Comfortable homes favour people's health.

This manifestation of comfort seems to be less important related to the domain of Acoustics. The reason for this is probably that, generally, the range of noises and sounds to which people are exposed to in their everyday life is not loud enough to cause physiological damage.

\section{PROTECTION FROM MOISTURE}

Environmental Cue related to the domain of Warmness

Humid locations can feel cold on the outside and thus people worry that the homes in those locations being cold. Barriers and mechanisms can help.

\section{PROXIMITY TO NEIGHBOURS}

Environmental Cue related to the domains of Warmness and Acoustics

Neighbours can be the source of certain nuisances that are particularly hard to control. Specifically, noise, privacy, and odours seem to be common concerns. This might be related to their pets (see the code "Pets" in this list) or to the neighbours themselves.

So, if neighbours are too close, people will infer that they will be exposed to some uncomfortable situations in the future.

\section{RELATIVE HUMIDITY}

Objective Indoor Climatic Factor related to the domains of Coolness and Air Quality

A high (perceived) relative humidity seems to be associated with poor air quality and a higher thermal sensation.

\section{SAFETY}

Trade-Off related to the domains of Acoustics and Coolness

People often want to open the windows for ventilating or cooling their homes. However, leaving the windows open is not always a good idea due to safety concerns (e.g., people can come in through the windows or people can fall out of them).

Similarly, while it is common to like quiet homes, a dwelling that is excessively quiet can make people feel disconnected from the other members of their household and, in consequence, make them feel unsafe.

\section{SENSATION WHEN ENTERING A SPACE}

Perception related to the domains of Daylight, Warmness, and Coolness

First impressions count. Even if they are brief, it is possible that the short experience of entering a comfortable space leaves some mark in people's overall assessment of such a space.

This might, or might not (depending on the domain), be related to the physiological phenomenon known as Alliesthesia. This is the pleasure felt when a certain external stimuli restores some internal variable that was misplaced. For instance, people often feel this when entering a warm space after walking in the cold for a relatively long time, and when being exposed to a cool breeze when feeling too hot. 


\section{SENSE OF COMMUNITY}

Perception related to the domain of Acoustics

Even if neighbours might be the source of some nuisances, people seem keen to be part of a community. This perception seems to be particularly (although not exclusively) produced by the sound that other people make performing recreational activities, such as playing, laughing, and practising sports.

\section{SENSE OF CONNECTION WITH THE EXTERIOR}

Perception related to the domain of Daylight

Having daylight allows people to connect with the exterior. It helps them feel how the time passes and to distinguish between daytime and night time.

It is possible that this perception is related to the reduced Feeling of Confinement-also influenced by Daylight-but confirming this requires further research.

\section{SENSITIVITY TO NOISE}

Internal Element related to the domain of Acoustics

Some people are simply more sensitive to noise than others. Whether this is because of some illness, the nature of their job, or simply because of personal preferences, understanding the specific requirements can be significantly relevant.

\section{SENSITIVITY TO ODOURS AND DUST}

Internal Element related to the domain of Air Quality

Some people are more sensitive to odours and dust than others. Reasons for this include a good/bad sense of smell, allergies, or simply caring more or less about it.

\section{SITE CHARACTERISTICS}

Environmental Cue related to the domains of Daylight, Warmness, Coolness, Air Quality and Acoustics

Not all sites in the same neighbourhood are the same. Obstructions-such as retaining walls, trees, or hills — can make a site less sunny while protecting it from the wind. Similarly, green areas are seen as beneficial for air quality and for preventing overheating. These cues can be read by people to infer what the future might be like.

This code is similar to the neighbourhood characteristics but at a smaller scale (i.e. site scale as opposed to urban scale).

\section{SIZE OF THE SPACE}

Environmental Cue related to the domains of Daylight, Warmness, Coolness, Air Quality and Acoustic

The size of the space affects our perception of it and also its thermodynamic and optical behaviour. Bigger spaces tend to be harder to warm up and cool down, and smaller spaces are associated with a Feeling of Confinement (which is related to Coolness). This characteristic is one of the first elements that people notice, sometimes influencings their assessment of comfort.

\section{STREETS NEARBY}

Environmental Cue related to the domains of Air Quality and Acoustic

Streets have cars, buses and trucks. These are noisy, dirty and smelly.

Not all streets are the same, though. People tend to know which streets are busier and which streets are quiet. 


\section{SYSTEMS THAT DO NOT FAIL}

Criterion for inferring Expected Outcomes related to the domains of Warmness and Acoustics

Hopefully, homes should be robust enough to consistently offer comfortable spaces for people. This implies, for instance, that heaters should fail as little as possible; and if they fail, this event should not prevent the whole house from being liveable. In a similar manner, windows, doors, roofs and other architectural elements of the dwelling should perform appropriately in a consistent manner.

\section{TEMPORARY}

Criterion for inferring Expected Outcomes related to the domains of Air Quality and Acoustics

Nuisances and uncomfortable situations can always happen. When there seem to be no means to solve them, the best people can do is to expect they finish soon. Knowing that something will_ or will not—end soon modulates people's Feeling of Comfort

\section{THE SOUND OF SILENCE}

\section{Perception related to the domain of Acoustics}

While, at first, people tend to say that absolute silence is what they want, it seems that they enjoy hearing natural sounds. Consequently, the sound of silence is not absolute silence, but just soothing-mostly natural—sounds.

\section{THERMAL COMFORT VS ACOUSTIC PERFORMANCE}

Trade-Off related to the domains of Acoustics and Coolness

People commonly open the windows when it is hot. However, sometimes this results in an even more unpleasant situation due to exterior noise.

\section{THERMAL COMFORT VS AIR QUALITY}

Trade-off related to the domains of Coolness, Warmness and Air Quality

Ventilating implies bringing fresh air into the home. Doing this during cold days can be a nuisance, as that air will be cold and might lower the temperature of the whole house. Something similar happens during hot days. The choices, then, are to ventilate and feel thermally uncomfortable, or not to ventilate and feel relatively fine. (This is, of course, assuming that it is possible to maintain the dwelling at a more pleasant temperature than the exterior).

\section{THERMAL INSULATION}

Environmental Cue related to the domains of Warmness, Coolness, and Air Quality

Whether they have been taught about this or they intuitively know it, thermal insulation is one of the characteristics that people use when making predictions about thermal comfort. Also, thermal insulation seems to be associated with dry dwellings; that is, dwellings that are not damp and mouldy.

\section{THERMAL MASS}

Environmental Cue related to the domain of Coolness

While probably related to (and, perhaps, sometimes confused with) thermal insulation, thick and heavy walls are noticed by people. These appear to be associated with homes that overheat less often.

\section{THERMAL PREFERENCE}

Internal Element related to the domains of Warmness and Coolness

While people tend to agree about the range of temperatures that are considered acceptable (i.e., somewhere around $18 \mathrm{C}$ to $26 \mathrm{C}$ ), choosing an exact temperature within this range is complicated. Thermal preferences vary within this relatively small range of temperatures. 


\section{THERMAL SENSATION}

Perception related to the domains of Warmness, Coolness and Air Quality

Thermal sensation is the name that has been historically given to the perception of how cold or hot is the space. It is usually measured using bipolar adjective (i.e. a Likert) scale that goes from "Too Cold" to "Too Hot" going through "Neutral".

It is very often assumed that "neutral" or "nearly-neutral" thermal sensations imply comfort. So, people's quotes gathered within this code include those referring to pure perception (e.g., "cold", "hot") and also to thermal preference (e.g., "pleasant temperature").

Since Thermal Sensation is a perception that considers the overall thermal environment, it might be necessary to consider that different parts of people's body might feel colder or hotter. This is reflected, for instance, in that the Floor Contact Temperature seems to be quite relevant for people.

\section{TIMES OF OCCUPANCY}

Internal Element related to the domains of Daylight, Warmness, Coolness, and Acoustics

The experience of people in a certain dwelling might be dramatically different depending on their routines and schedules. A retired person, for instance, might want to have daylight all day. Whereas a busy person who gets home late and only sleeps there might favour mornings or evenings.

\section{UNCOMFORTABLE AMOUNT OF CLOTHING}

Trade-Off related to the domains of Coolness and Warmness

While people can feel warmer by wearing more layers of clothing, they do not always do it happily. Sometimes people feel that they need to wear more layers than what they find appropriate. The same happens during hot days, when they can potentially take some more clothes off but they hesitate. The options are, then, to wear comfortable clothing while feeling relatively uncomfortable, or to wear more appropriate clothing while feeling more comfortable.

\section{UTILITY BILLS}

Trade-off related to the domains of Daylight, Warmness and Coolness

Utility bills that are too expensive can prevent people from heating and cooling, thus negatively affecting their living experience. Some people will spend whatever amount of money is required to be comfortable, but others will simply sacrifice their level of comfort and avoid spending more than what they have budgeted to this field.

This is related to people's Budget (see code with that name).

\section{VENTILATION SYSTEM}

Environmental Cue related to the domain of Air Quality

Windows are good for air quality. But they are not always available or sometimes they are not effective enough. A mechanical ventilation system-e.g. an exhaust fan-can help.

It is worth noticing that some people do not trust these systems too much, which is why they favour opening windows.

\section{VIEWS}

Environmental Cue related to the domain of Daylight

The availability of windows and skylights not only provides daylight but also views. This was identified by some respondents, who argued that dwellings with good daylight also have views. 
There is a self-evident correlation between views and daylight. However, it is unclear whether different views to outside (more open, obstructed, rural or urban) will affect the perception of brightness of the space, or the satisfaction with it in terms of visual comfort.

\section{WEATHER SOUNDS}

Environmental Cue related to the domain of Warmness

The amount of noise or sound from the exterior-e.g. rain, hail, wind-seems to influence people's Feeling of Comfort. This appears to be related to expected outcomes; specifically, to the fear of the envelope failing to protect them from the external elements. 
Appendix V: Overview of the routines utilized for the simulation in Section 8.4

/// This function runs the simulation

/// For the sake of clarity, not everything is shown

/// This is written in the Rust programming language.

/ / /

/// More details available at

/// https://github.com/germolinal/PhD_Thesis_Simulations

fn main( ) \{

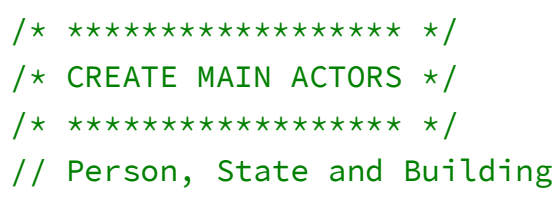


// Too much and too little clothing are equally bad

person.add_perception(

poly! [๑.๑, $\odot . \odot,-1.5], / / \odot+\odot * x-1.5 * x^{\wedge} 2$

Perception: : ClothingAnnoyanceTooMuch

) ;

person.add_perception(

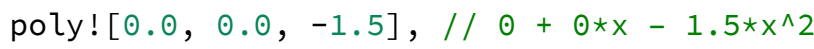

Perception: : ClothingAnnoyanceTooLittle

) ;

// Too much and too little Loudness are equally bad

person.add_perception(

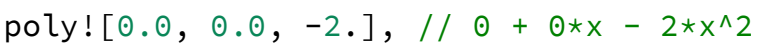

Perception: : LoudnessTooMuch

);

person.add_perception(

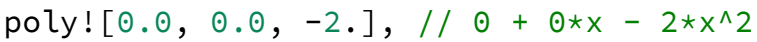

Perception: : LoudnessTooLittle

)

// Brightness is good (more is better)

person.add_perception(

poly! $[\odot . \odot, 5 . \odot], / / \odot+5 * x$

Perception: : Brightness

) ;

// Utility bills are bad.

person.add_perception(

poly! $[\odot . \odot, \odot . \odot,-\odot .1], / / \odot+\odot * x-\odot .1 * x^{\wedge} 2$

Perception: :UtilityBills

) ;

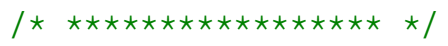

/* DEFINE BUILDING $\star /$

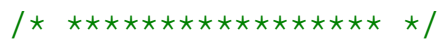

// For the sake of clarity and briefness, this is summarized

// in this way.

//

// This function defines a 2-Bedroom + Livingroom + Bathroom + Kitchen

// + Hallway home. All walls are made of $180 \mathrm{~mm}$ concrete, and the windows

// are 3mm glass.

//

// Every space has openable windows, a 1500W heater and 180W of

// switchable lights

create_building(case, \&mut building, \&mut state); 


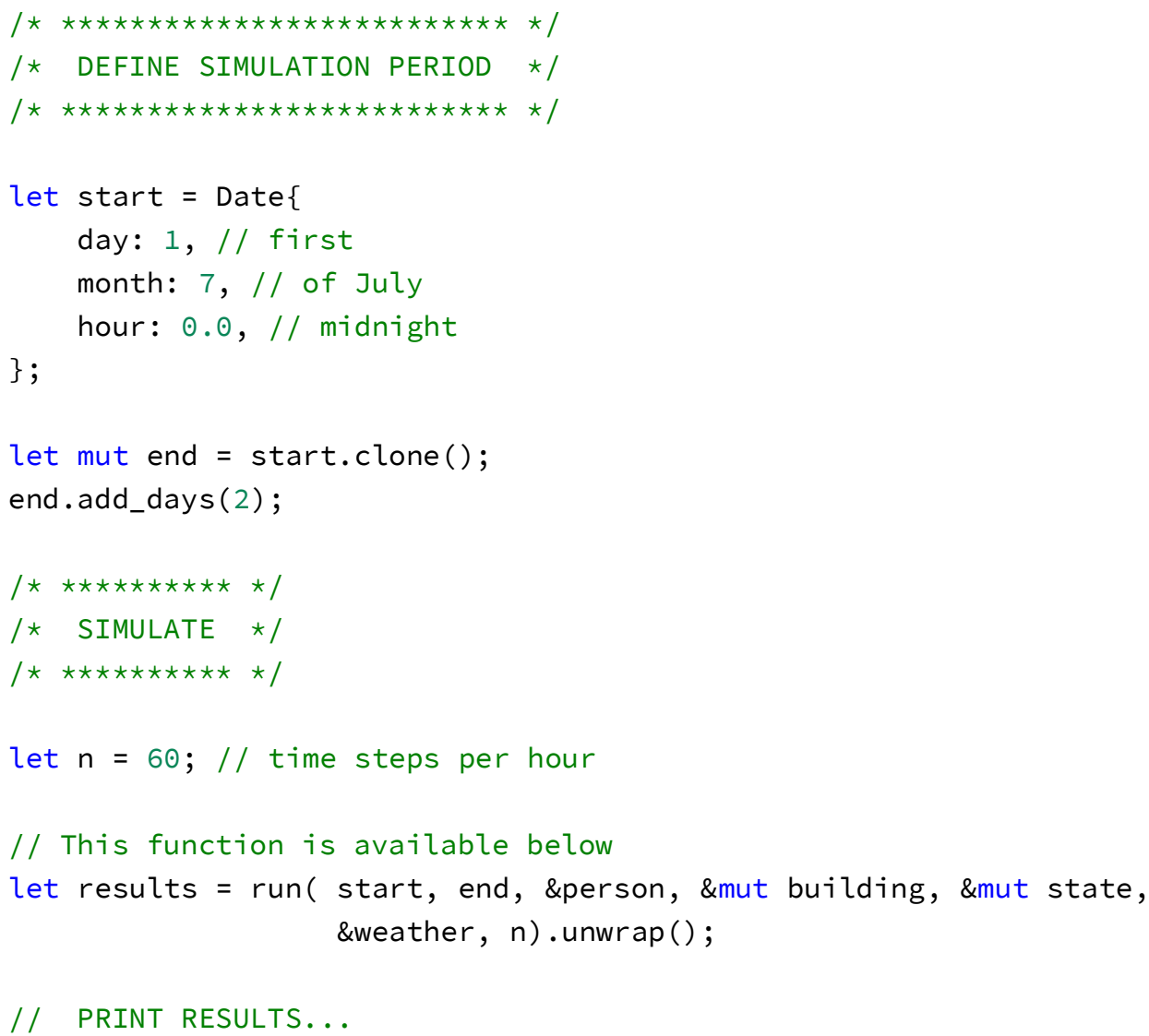




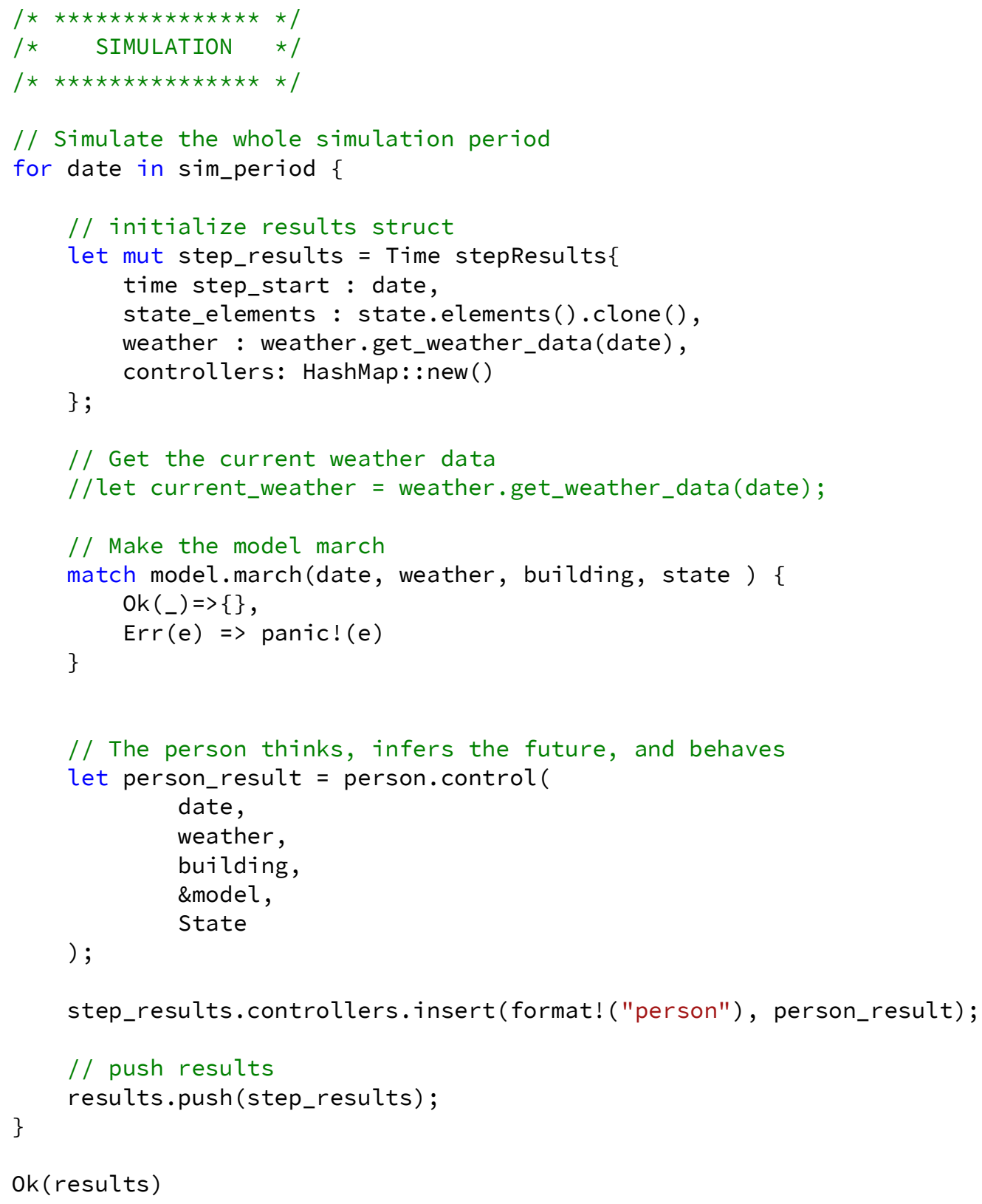

